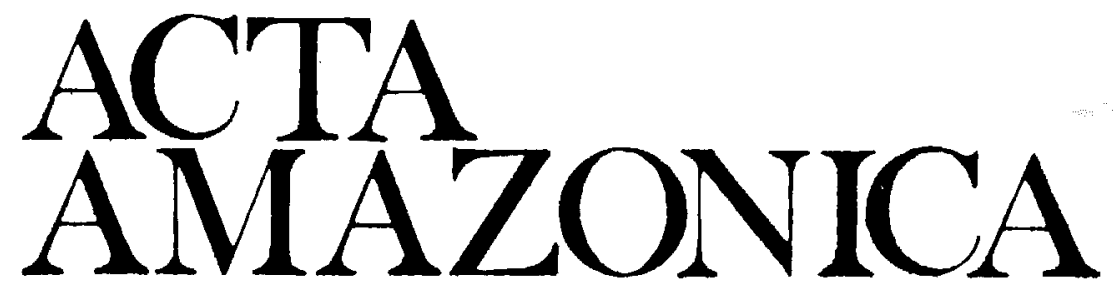

Número - 3/4

Volume 15

Suplemento

Set / Dez -1985

\title{
Habitat preferences, diet, feeding strategy and social organization of the black spider monkey [Ateles paniscus paniscus Linnaeus 1658] in Surinam
}

M. G. M. van Roosmalen *

* This field study of the black spider monkey was financed by the Netherlands Foundation for the Advancement of Tropical Research, The Hague. Departmental facilities were kindly provided by the Research Institute for Nature Management, the Institute of Systematic Botany, Utrecht, and the Agricultural University, Wageningen. 
ABSTRACT (back cover)

HABITAT PREFERENCES, DIET, FEEDING BEHAVIOR AND SOCLAL ORGANIZATION OF THE BLACK SPIDER MONKEY, ATELES PANISCUS PANISCUS, IN SURINAM.

\section{A Socioecological Field Study.}

This monograph reports on a 26 month socioecological study of black spider monkeys (Ateles paniscus paniscus) in the Raleighvallen - Voltzberg Nature Reserve, Surinam. It recognizes the fundamental importance of food to the behavior and the regulation of population density for this primate. It clarifies the complex temporal and spatial effects of tropical rain forest food sources on the behavior of a group of spider monkeys, concentrating on food category, food plant identity and phenology, and quantity, density and dispersion of the most important food sources. In addition, the present study describes habitat choice, optimal feeding strategy and sexual behavior of the spider monkey, and discusses implications of diet for social behavior. This study is also fundamental to conservation. Specialized in eating mature fruits, the spider monkey is a very important dispersal agent for many trees and lianes, particularly ca. nopy species. However, the spider monkey is probably the most vulnerable monkey species in Surinam and it is disappearing rapidly throughout the remainder of its range. Unfortunately, it is large and noisy and can be easily tracked and hunted. It is largely restricted to undisturbed high forest, and consequently habitat destruction has more effect on it than on most other species. Together with its slow reproductive rate (a female gives birth only once every four or five years), this means that the species is poorly adapted to recover from exploitation. In order to implement proper measures for conservation, data on forest type preferences, diet and social behavior of the species, or on closely related species, in undisturbed axeas, such as the one described in this monograph, are essential tools for assessing the potential of proposed protected areas.

M.G.M. van Roosmalen (Leersum, Holland - Manaus; Brazil) Habitat Preferences, Diet, Feeding Behavior and Social Organization of the Black Spider Monkey, Ateles paniscus paniscus, in Surinam, 42 fig., 14 tables, 1986. 


\section{CONTENTS}

Acknowledgements

\section{Introduction}

Background .............................................................. 9

Taxonomy and Distribution of Ateles............................ 11

Population Density and Biomass............................... 12

Climate .............................................................. 15

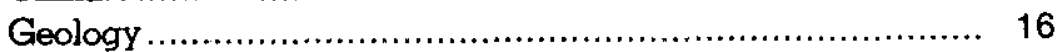

Topography....................................................... 18

Vegetation of Surinam.......................................... 18

Structural Description of the Forest Types found in the

Raleighvallen-Voltzberg Region.............................. 18

Dry Evergreen Forest Types.................................. 18

Wet Forest Types............................................... 28

Xeromorphic Vegetation.................................... 30

\section{Methods}

The Study Area ......................................................

Data Collection................................................. 37

Habituation of Spider Monkeys............................. 37

The Synecological Part..................................... 38

The Autecological Part........................................ 40

The General Survey................................................ 44

Lolopasi, West Bank Coppename River, Raleighvallen-

Voltzberg Nature Reserve....................................... 44

Kayser Moutains................................................ 44

Other Parts of Surinam....................................... 45

\section{Habitat Preferences}

Preferences for Different Forest Types............................ 46

Edge Preferences.................................................. 48

Vertical Stratification................................................ 49 


\section{Diet and Feeding Behavior}

Food Categories................................................. 50

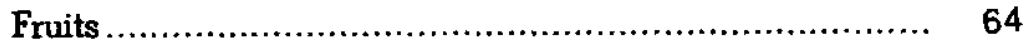

Flowers.............................................................. 73

Leaves............................................................. 74

Pseudobulbs ..................................................... $\quad 75$

Aerial Roots.................................................... 75

Bark and Decaying Wood.................................... 75

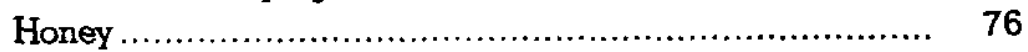

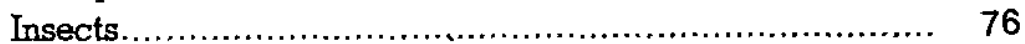

Feeding Behavior ............................................ 77

Foraging Techniques....................................... 81

Feeding Techniques........................................ 83

Feeding Heights............................................ 86

General Feeding Postures.................................. 86

Food Selectivity ............................................. 87

Temporal Patterning of Food Choice......................... 87

Diumal Variation in Food Choice............................ 89

Variation in Food Choice between Days..................... 90

Monthly Variation in Food Choice.............................. 101

Monthly Variation in Food Supply........................... 137

Food Supply and Food Choice................................. 139

Ateles as a Specialized Frugivore............................... 140

\section{Social Organization}

Introduction ....................................................... 147

Group Size, Group Composition and Age Classes............. 148

Group Range and Day Range Length......................... 150

Grouping Behavior................................................. 151

Male Behavior................................................... 166

Calling Behavior.................................................... 168

Sleeping Behavior................................................... 171

Reproductive Biology and Sexual Behavior.................... 175

6. Conservation ............................................... 179

7. Discussion................................................. 181

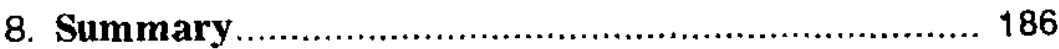

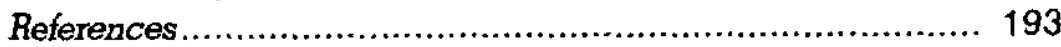

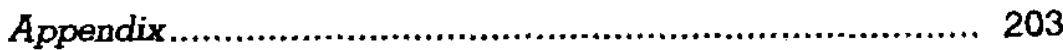




\section{ACKNOWLEDGEMENTS}

I am indebted to a number of people who made this study possible. First of all I would very much like to express my thanks to my friend and colleague Dr. Russell A. Mittermeier, who introduced me to the tropical rain forest and the world of Surinam monkeys. Together we set up an area for studying the synecology of Surinam monkeys, and the same study area was later used for the autecological study of spider monkeys described in this monograph. His assistance in the field during part of the study and his valuable discussions and critical comments, both in the field and during the analysis and writing, have been essential for my work.

Special thanks are also due to Dr. Anne van Wijngaarden, who supervised the project in all its stages, for his practical advice and encouragement and for contributing many useful comments. Without the continual support of these two people the project could never have been carried out.

I also wish to thank Prof. Dr. M.F. Mörzer Bruyns of the Agricultural University, Wageningen, Prof, Dr. F.S. Szalay,Dr. John G. Fleagie, Dr. John F. Oates, Dr. Katharine Milton and Dr. Jan C. Lindeman for their constructive comments on the draft of this manuscript.

Thanks are also extended to Dr. A. Gentry of the Missouri Botanical Garden for identifying several samples of rare Bignoniaceae; to Mrs. Marieke E. van Roossmalen for comments on chapter 5; to Dr. Nicole Duplaix for locality data and other information on spider monkeys; to Dr. Jan C. Lindeman, Mrs. M. Werkhoven and Mr. P. Teunissen for valuable information on Surinam forest types and plant species; to the Surinam Geological Services for transportation and lodging in the Kaiser Mountains, where I carried out a survey using their trail system; to Mr. Paul Loth for assisting me in the field during the last seven months and to Mr. Frits van Troon, treespecialist of Dienst's Lands Bosbehee, for marking about 10,000 trees and lianes in the study area.

I am greatly indebted to my Surinam Bushnegro and Amerin. dian friends and their children on Foengoe Island, Raleighvallen. They provided us, and particularly my wife and son, a very pleasant atmosphere in which to live during the course of the field study. 
Many thanks go to Mr. Arjan Griftioen, the artist of the Research Institute for Nature Management, Leersum, for preparation of the histograms and maps.

I shall always be much indebted to my wife Betty for the unselfish contribution she has made to every aspect of this long study. Moreover, most drawings of food items were made by her in the field. This field study of the black spider monkey was financed by the Netherlands Foundation for the Advancement of Tropical Reseanch, The Hague. Departmental facilities were kindly provided by the Research Institute for Nature Management, the Institute of Systematic Botany, Utrecht, and the Agricultural University, Wageningen. 


\section{INTRODUCTION}

\subsection{Background}

The role of food as a basic determinant of social arganization and behavior has been a much discussed topic in the recent literature on free-ranging primates (Chivers, 1977; Clutton-Brock, 1974; Rasmussen, 1979; Rodman, 1973, 1977; Wrangham, 1977, 1979). Unfortunately, the detailed autecological studies on undisturbed primate populations urgently needed for this investigation are few in number. One of the main reasons may be the fact that primatology and botany are a rare combination of skills. The floristic complexity of the oldest terrestrial ecosystem on earth, the tropical rain forest, surely plays a role as well. Recognizing this before starting the present field sdudy, I compiled all available data on truits and seeds of woody plants of Surinam since it was known that all monkeys occuring in the country are at least partly frugivorous (Husson, 1957). Two years of research resulted in a book (Surinaams Vruchtenboek, 1977) with detailed descriptions of the fruits and seeds of about 1400 species of tree and liane. In most cases a drawing was included, in addition to general descriptions of leaves and inflorescences of the species, habit of the plant and habitat preferences. This book turned out to be a useful guide for identifying most food plants in the field. Feeding monkeys drop fruit parts and sooner or later complete fruits, sometimes with leaves attached, whereas trees and lianes usually drop their fruits in a certain stage of maturity, making the collecting of fruits much easier than that of flowers and leaves.

The identification of species in the field on fresh samples increases one's knowledge of the forest much faster than the usual way of collecting, preserving, shipping and, much later, identifying the samples. Knowledge of plants is of great advantage to studies of monkeys. Types and subtypes of forest are easier to recognize by determining and recognizing certain indicator species. One's understanding of the forest is better when one is no longer walking in a highly variable mass of trunks and stems. By fixing in one's memory special properties of individual trees of known species, one 
becomes habituated to every part of the forest previously visited. All one's attention can be directed to the monkey's behavior. Also the phenology of forest plants can easily be studied by collecting all kinds of fresh fruit from the forest floor from month to month in successive years. In this way periodicity and length of fruiting period. can be determined for many species, both at individual plant and species level.

Until recently, very little information was available on diet and habitat choice of Ateles paniscus paniscus. More is known about two other Ateles species, $A$. belzebuth and A. geoffroyi.C.R. Carpenter (1935) reported on a natural population of $A$. geoffroyi in Panama. He described the species as frugivorous. About $90 \%$ of the items consumed were said to be fruits. Little data on other food stuffs were reported by Carpenter. In 1969, Hadik and Hladik described feeding behavior, diet and quantitative and chemical analyses of many food items used by a group of $A$. geoffroyi, reintroduced to Barro Colorado Island, Canal Zone, in 1961. Since their study did not cover an entire year, it is difficult to interpret their results. Moreover, the monkeys lacked the traditionally developed knowledge of food and optimal feeding strategy as found in wild populations. Klein and Klein observed free-ranging $A$. belzebuth in the Colombian National Park, La Macarena, during one year (1967-1968). Among other things, they collected information on diet, feeding behavior, feeding heights and sites and social organization (1972, 1976, 1977).

The present study recognizes the fundamental importance of food both to the behavior and the regulation of population density for the black spider monkey in Surinam. It clarifies the complex temporal and spatial effects of food souices on the behavior of a group of spider monkeys, concentrating on food category, food plant identity and phenology, and quantity, density and dispersion of the most important food sources. In addition, this study describes habitat choice, optimal feeding strategy and sexual behavior, and discusses implications of diet for social behavior. This study is also fundamental from the viewpoint of conservation. Ateles is probably the most vulnerable monkey species in Surinam. It is large and noisy and can be easily tracked and hunted. In undisturbed areas, it usually reacts to a human intruder by performing a 'branch shaking and branch 
dropping display', which attacts other nearby subgroups. A hunter can wipe out most of the group with little effort. Moreover, Ateles is largely restricted to undisturbed high forest, and habitat destruction has more effect on it than on most other species. Another important point is its slow reproductive rate. A female usually gives birth only once every four or five years, meaning that the species recovers slowly from exploitation. In order to implement proper measures for conservation, data on forest type preferences and diet of the species that are to be protected, are needed since they are essential tools for assessing the potential of proposed protected areas.

\subsection{Taxonomy and Distribution of Ateles}

The taxonomy of the genus Ateles is the subject of some controversy. Kellogg and Goldman (1944) recognize four species of Ateles: A. geoffroyi, $A$. fusciceps, $A$. belzebuth and $A$. paniscus. Several recent authors (e.g. Hershkovitz, 1972; Groves, 1972; Moynihan, 1970, 1976), however, consider all Ateles conspecific and refer to them collectively as $A$. paniscus (the oldest available name). Recent studies indicate that there may be some $1-2$ chromosome significant differences among the species recognized by Kellogg and Goldman (Heltne and Kunkel, 1975). Furthermore, an important contact zone between $A$. geoffroyi and $A$. fusciceps exists in eastern Panama and the species apparently hybridizes to some extent (Rossan and Baerg, 1977). Until further information becomes available, I preter to follow Kellogg and Goldman (1944) and use the name Ateles paniscus in the strict sense.

The Surinam representative of the genus is Ateles paniscus paniscus (Linnaeus, 1758). It is found in lower Amazonia, between the Rio Negro and the Atlantic and north to the Guianas (Kellogg and Goldman, 1944), a range similar to that of Saguinus midas midas, Pithecia pithecia, Chiropotes satanas chiropotes and Cebus nigrivittatus, four of the other seven primate species occuring in Surinam. The second $A$. paniscus subspecies, $A$. p. chamek, is found in western Matto Grosso, eastern Bolivia and northeastern Peru and extends into Brazil as far east as the Rio Junua, a southem tributary of the Rio Amazonas (Kellogg and Goldman, 1944). The other three species of Ateles are found from Southern Mexico to the southern reaches of the Amazon basin. 
In Surinam, A. p. paniscus is almost entirely restricted to the interior. Like Chiropotes s. chiropotes and Cebus nigrivittatus, it just enters the old coastal plain in the western part of the country (fig. 1). Its is covered with long, glossy black hair. The naked face varies from light to dark pink and is sometimes lightly freckled (fig. 2). The eyes are usually brown, but some individuals have blue eyes. The female has a long pendulous, backward-directed clitoris, that immediately distinguishes her in the field. The scrotum of the male is black. The hair on the head in both sexes is long and directed forward, forming a peak over the eyes (fig. 3). Individual difference in face color and marking, eye color, hair tufts, hair length and physique make the animals readily recognizable in the field, even at great distances. Ateles p. paniscus is a large monkey. Three males in the British Museum had a meanisc head-body length of $545 \mathrm{~mm}$ (range: 515-580) and a mean tail length of $807 \mathrm{~mm}$ (range: 720-852); ten females had a mean head-body length of $540 \mathrm{~mm}$ (range: 490-620) and a mean tail length of $814 \mathrm{~mm}$ (range: 640-930) (Napier, 1976). Four females cited in Kellogg and Goldman (1944) had the follo. wing measurements: head-body length: $460,418,570$ and $660 \mathrm{~mm}$; tail lenght: $870,920,880$ and $753 \mathrm{~mm}$. Adult males usually are more heavily built, but sometimes extremely large females have been observed too. Five males weighed in Surinam had a mean body weight of $7.86 \mathrm{~kg}$ (range: $6.5-9.2$ ) and seven females a mean body weight of $7.70 \mathrm{~kg}$ (range: 6.5-8.5) (Mittermeier, 1977).

\subsection{Population Density and Biomass}

Because of flexible grouping behavior in Ateles, accurate estimation of group size, group composition and population density is only possible if the members of a group are recognizable as individuals. In the Voltzberg area in Surinam, A. paniscus occurs at a density of 7.1. individuals or 6.3 independently locomoting individuals per $\mathrm{km}^{2}$. When only the area of suitable habitat is considered, an economic density of 8.2 , resp. 7.3 individuals per $\mathrm{km}^{2}$ results. The figures from other areas vary considerably, but only the estimate of Klein and Klein (1976) is based on individually recognizable animals of a natural population. Muckenhim, et al. (1975) estimate 2.4-6.2 individuals per $\mathrm{km}^{2}$ for $A$. p. paniscus in Guyana; Heltne, et. al. (1975) give 2.0 individuals per $\mathrm{km}^{2}$ for $A$. p. chamek in Bolivia; Klein and Klein (1976) give a density of 12-15 indi- 


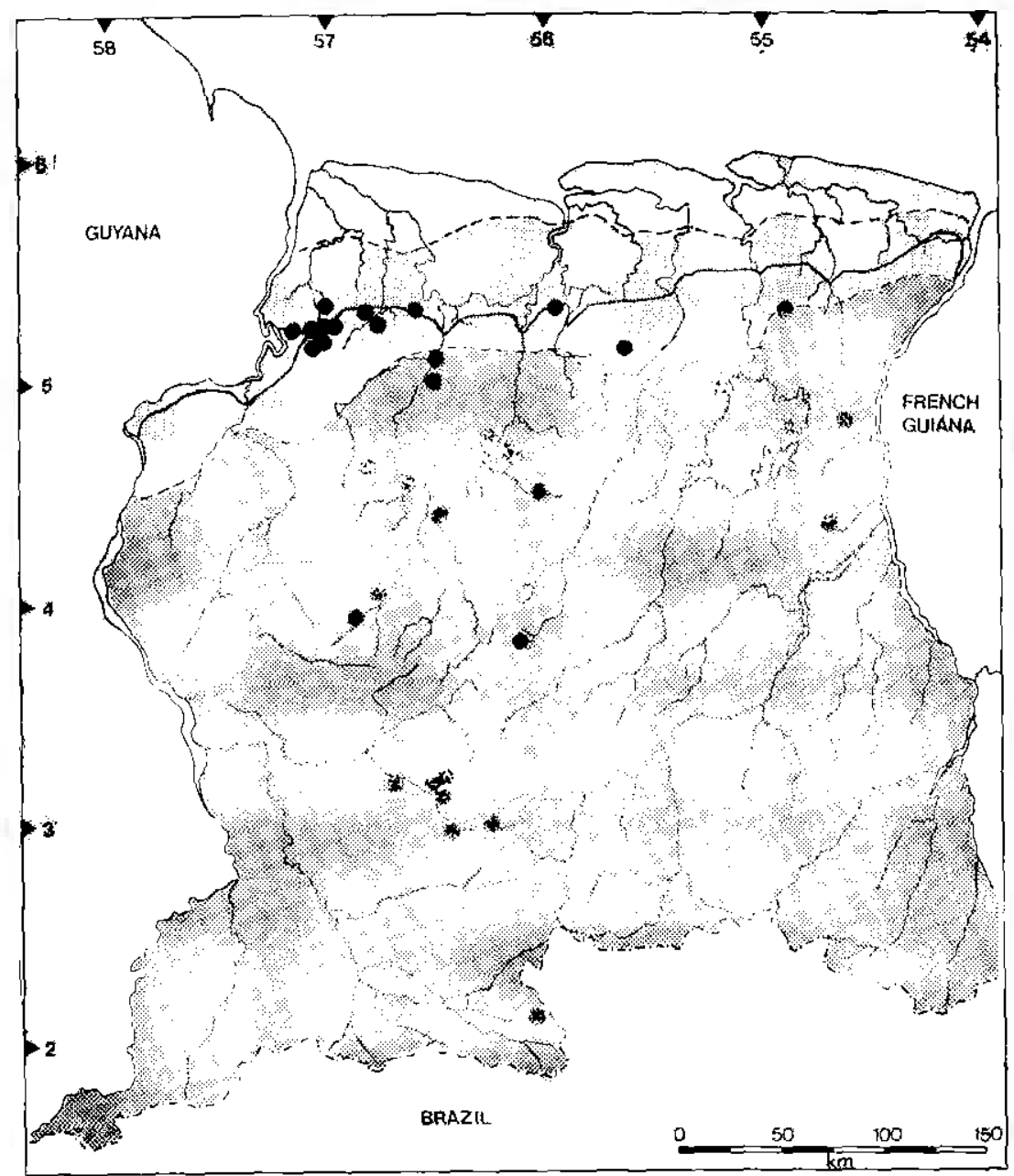

Young coastal plain

old coastal plain + savanna belt

- ATELES PANISCUS PANISCUS

\section{Interior}

Figure I Distribution map for Ateles paniscus paniscus in Surinam. The central broken line marks the border between the coastal region and the interior. The area immedia. tely to the north of this line is the old coastal plain, that to the south is the savan. na belt. The dashed lines indicate the borders of the two areas. For the purposes of this study, the savanna beit is considered part of the interior, but it is actually a geological distinct region. Ateles occurs throughout the interior, but is rare in the savanna belt and only enters the old coastal plain in the extreme western part of the country. 


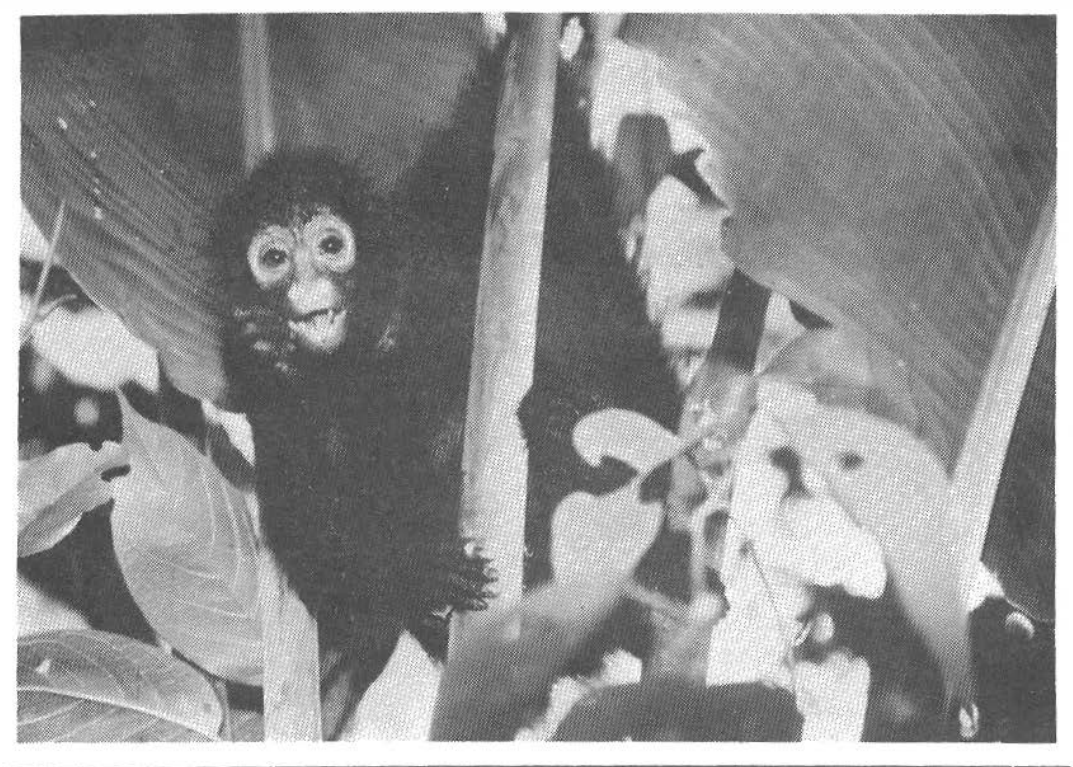

Figure 2 Juvenile-1 Ateles $p$ paniscus trom Surinam. The naked face is light pink and may be lightly freckled.

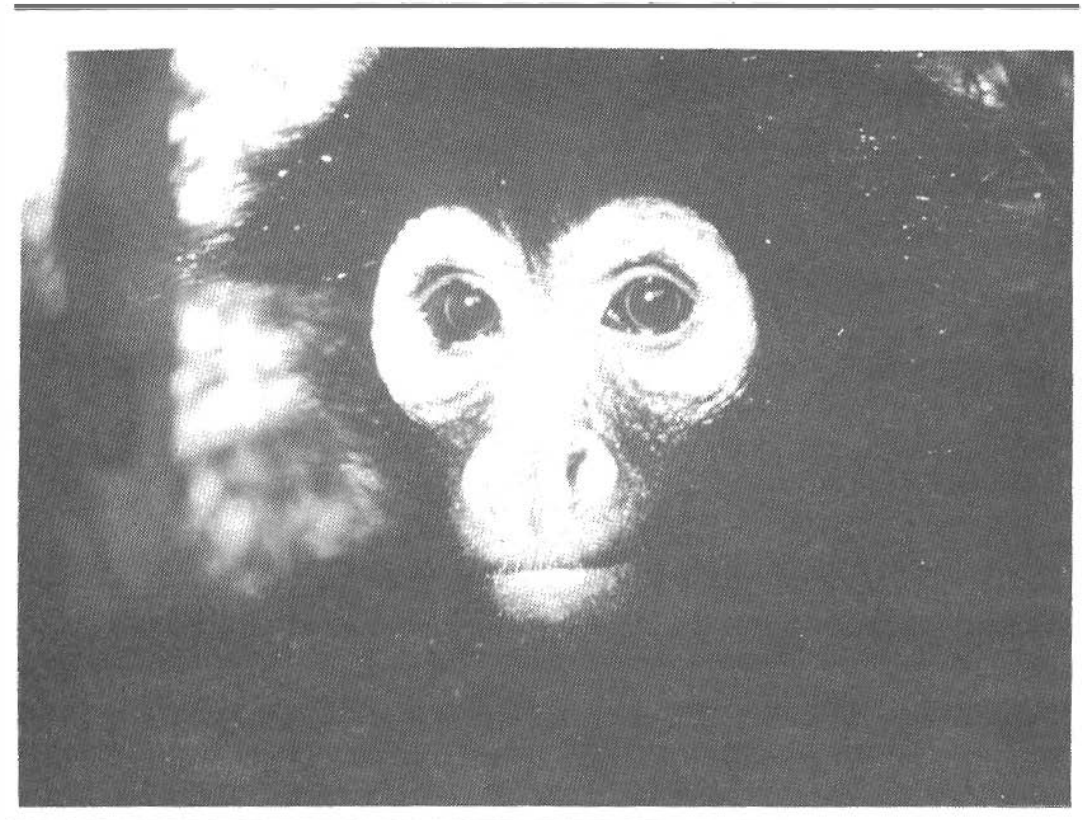

Figure 3. Adult temale Ateles p paniscus trom Surinam (Photo by R. A. Mittermeier). 
viduals of one year or older per $\mathrm{km}^{2}$, and including infants 15-18 individuals per $\mathrm{km}^{2}$ for. $A$. belzebuth in Colombia; Bernstein, et. al. (1976) estimate 9-14 individuals per $\mathrm{km}^{2}$ in northem Colombia; Freese (1976) found 6-9 A. geoffroyi per $\mathrm{km}^{2}$ in Costa Rica. In Guatemala, Coelho et al. (1976) estimated 45 individuals per $\mathrm{km}^{2}$ and Cant (1978) 26 individuals per $\mathrm{km}^{2}$. In Peru, Janson (1975) estimated 24 A.p. chamek per $\mathrm{km}^{2}$.

The biomass for $\boldsymbol{A}$. paniscus in the Voltzberg area in Surinam is calculated using the mean body weigth data derived from Mittermeier (1977): $7.86 \mathrm{~kg}$ for males and 7.69 for females. The figures range between 0.4 and $0.5 \mathrm{~kg} / \mathrm{ha}$, depending on the home range size chosen (255 and $220 \mathrm{ha}$, resp.). Estimates from other areas are as follows: 0.07 (Eisenberg and Thorington, 1973), 0.2 (Heltne et al., 1975), 1.4-1.9 (Janson, 1975), 0.3-0.6 kg/ha (Muckenhim, et al., 1975), 0.7 (Bernstein, et al., 1976), 1.5 (Coelho, 1976), 0.3-0.5 (Freese, 1976), 0.6-0.9 (Klein and Klein, 1976), and $1.4 \mathrm{~kg} / \mathrm{ha}$ (Cant. 1978).

\subsection{Climate}

Surinam lies close to the equator and has a typical tropical climate. The mean annual temperature is $26.1^{\circ} \mathrm{C}$ (Lindeman and Moolenaar, 1959). Mean monthly temperatures vary only about 2 . during the year. A maximum is reached in September and October and a minimum in January and February. daily variations are greater and range from $21.0-31.6^{\circ} \mathrm{C}$ at $1.5 \mathrm{~m}$ in the rain forest. Temperatures at the top of the canopy are similar to those in clearings and range from $21.0-36.0^{\circ} \mathrm{C}$. Personally collected data over two years at $1.5 \mathrm{~m}$ in the rain forest in the Voltzberg area show temperature maxima in September, October and November and minima in June, July and January (Fig. 4).

Relative humidity in the rain forest is highest in the early morning ( $95 \%$ or more), drops to about $82 \%$ during the middle of the day and reaches $95 \%$ again at dusk. In clearings and at the top of the canopy the daily range is greater and humidity may be as low as $40 \%$ in the middle of the day (Schulz, 1960; Hoogmoed, 1969).

Mean annual precipitation lies between 2,000 and 2,400 mm (Iindeman and Moolenaar, 1959) and is not evenly distributed 
throughout the year. Usually, four seasons are recognized. A long wet season, usually beginning in mid-April and lasting until midJuly, with maximum rainfall in May or June. A long dry season begins in August and lasts until mid-November, October is, in the average, the driest month, but differs only slightly from September. The period from December to April can be divided into a short wet and a short dry season that vary consideraily in length and intensity. The shart wet season usually runs from December to mid-January or February, the short dry season from February to April. In abnormal years one of the two can be absent. In 1976, the long dry season lasted from August to 15 February 1977. Then, after a two-week wet period, a short dry season followed lasting six weeks, while the long wet season started about mid-April. Many trees dropped their leaves completely, giving a dead appearance to the mountain savanna forest. The long dry season is characterized by a monthly rainfall of less than $100 \mathrm{~mm}$. October is the driest month with as little as $20 \mathrm{~mm}$ locally (Hoogmoed, 1969). May is usually the wettest, with precipitation that may exceed $400 \mathrm{~mm}$ locally. During the two years of observation in the Voltzberg area, the driest month was September with precipitation as low as $68 \mathrm{~mm}$. The wettest month was May with $316 \mathrm{~mm}$ (fig 4).

The nights in the interior are mostly windless. Gales are rare. As elsewhere in the tropics the most violent winds are squalls. They occur during the transition periods (particularly in July and August) and may precede thunderstorms. During such squalls it is risky to walk through the forest. Old trees can be heard falling every where, especially during and just after heavy rainfall, and even younger vigorous trees may fall. This phenomenon of tree-fall is of great importance in the regeneration of primary forest.

\subsection{Geology}

The interior of Surinam is a part of the Guayana Shield, a formation of Precambrian age that is composed mainly of granitodiorites and granites. In central Surinam, a single remnant of the Mesozoic Roraima sandstones, the Tafelberg, has withstood erosion. The rocks in the interior are usually covered with deeply weathered ferrosiallitic/ferrallitic soils that vary from loamy sand to clay. Here one finds high upland forest. On shallow soils covering laterite caps and outcrops of unweathered gra nite, one finds more or less 
xeromorphic types of vegetation (Bakkes, 1957). In some areas, such as the Voltzberg region, exposed unweathered rock can still be seen at the surface. These granite-inselbergs are mainly covered with lichens and algae, which cause weathering with high $\mathrm{PH}$-values. The $\mathrm{SiO}_{2}$, dissolved under these circumstances is deposited in the form of small, very resistant sheets. Together with the lichens and algae, the $\mathrm{SiO}_{2}$ - sheets shut the granite off trom deeper weathe-

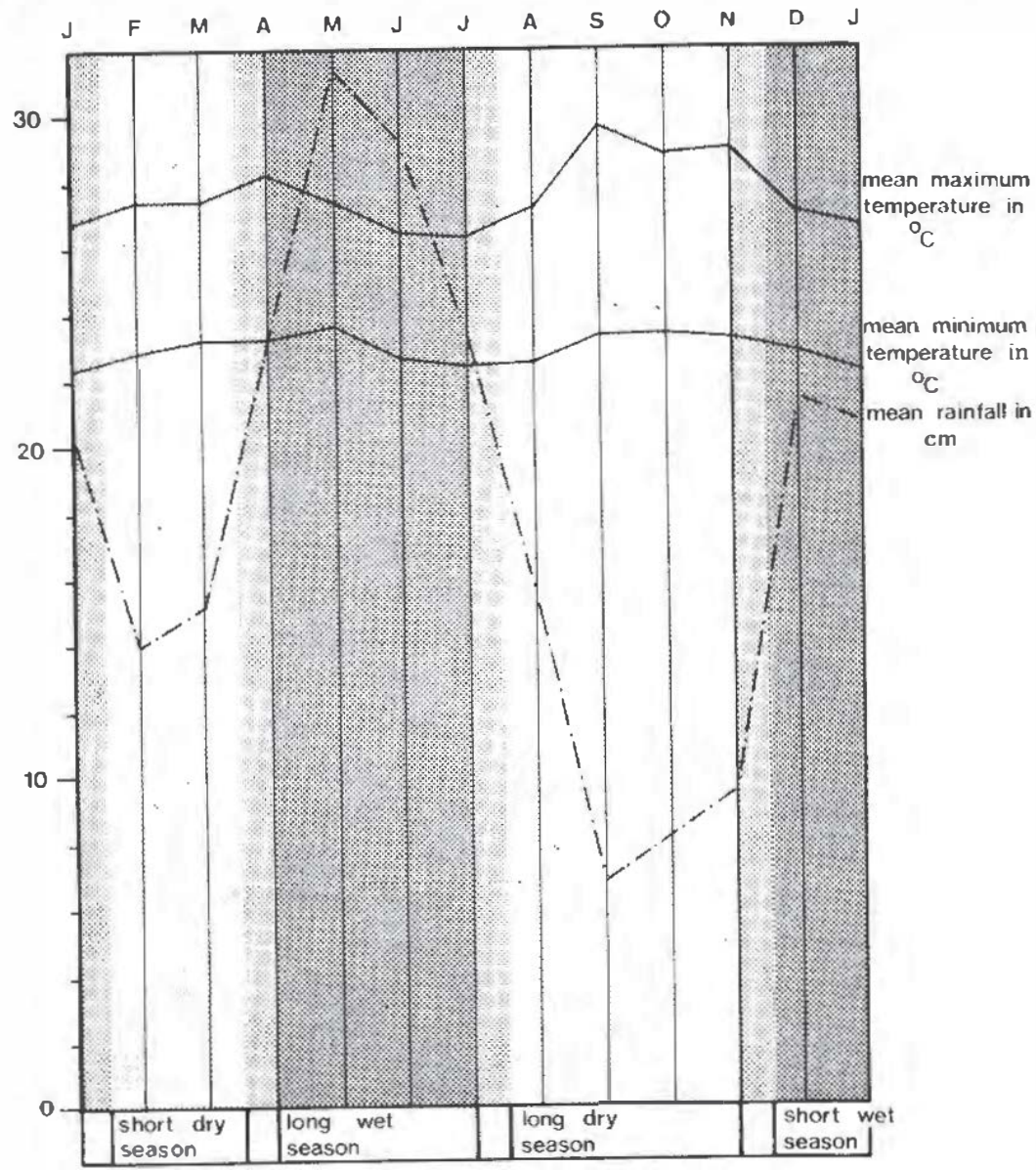

Figure 4 Climate of the Voltzberg study area during the present study, showing the monthly variations of mean maximum and minımum temperature at $1.5 \mathrm{~m}$ in the rain 10 rest (fall lines) and rainfall in a clearing (broken line). Data were collected from May 1976 to May 1978. 
ring, a process which seems to be essential in the forming of inselbergs in the tropics (Bakker, 1957).

\subsection{Tbpography}

The coastal region of Surinam has almost no relief. In the interior several low mountain ranges are found, the highest peak being $1,280 \mathrm{~m}$ (Wilhelmina Mountains). Several plateaus, for instance the Brownsberg $(514 \mathrm{~m})$, are given mountain status in Surinam, as are isolated outcroppings such as the Voltzberg $(240 \mathrm{~m})$ and the Van Stockumberg $(360 \mathrm{~m})$.

Six major rivers dissect the country and for the most part flow from south to north. The Corantyn formes the border between Surinam and Guyana, the Marowijne divides Surinam and French Guiana (fig. 5). The lower reaches of the rivers are calm, but the upper reaches below $5^{\circ} \mathrm{N}$ are braken by marry rapids (eg., the Ralejghvallencomplex in the Coppename River near the Voltzberg).

\subsection{Vegetation of Surinam}

The Surinam coast primarily consists of mudflats covered by mangroves which are broken by arcasional beaches. Behidind these lie large stretches of swamps, cut by sand and shell ridges running parallel to the coast. Moving further south, there is a belt of rain and marsh forests, then a narrow belt of white sand savannas and savanna forests, and finally the rain forests of the interior (Hoogmoed, 1969). The savanna belt, which begins roughly $25 \mathrm{~km}$ from the coast in the east and $80 \mathrm{~km}$ in the west, is not part of the coastal region and can be considered a division of its own or part of the interior. The interior is for the most part covered with high tropical rain forest broken by other forest types and small savanna enclaves. The largest of these is the Sipaliwini Savanna, which is located in the extreme south and connected with the larger Paru Savanna of Brazil (fig. 5).

\subsection{Structural Description of the Forest Types found in the Raleighvallen-Voltzberg Region.}

\subsubsection{DRY EVERGREEN FOREST TYPES}

\section{Tropical Rain Forest}

High rain forest. The range of forest types in Surinam covers Beard's (1955) categories of true tropical rain forest and evergreen seaso- 
nal forest. Since there is a continuum between the two categories, it is preferable to follow Lindeman and Moolenaar (1959) and Richards (1952) in using the term high rain or high forest, dryland forest to include the two Beard types.

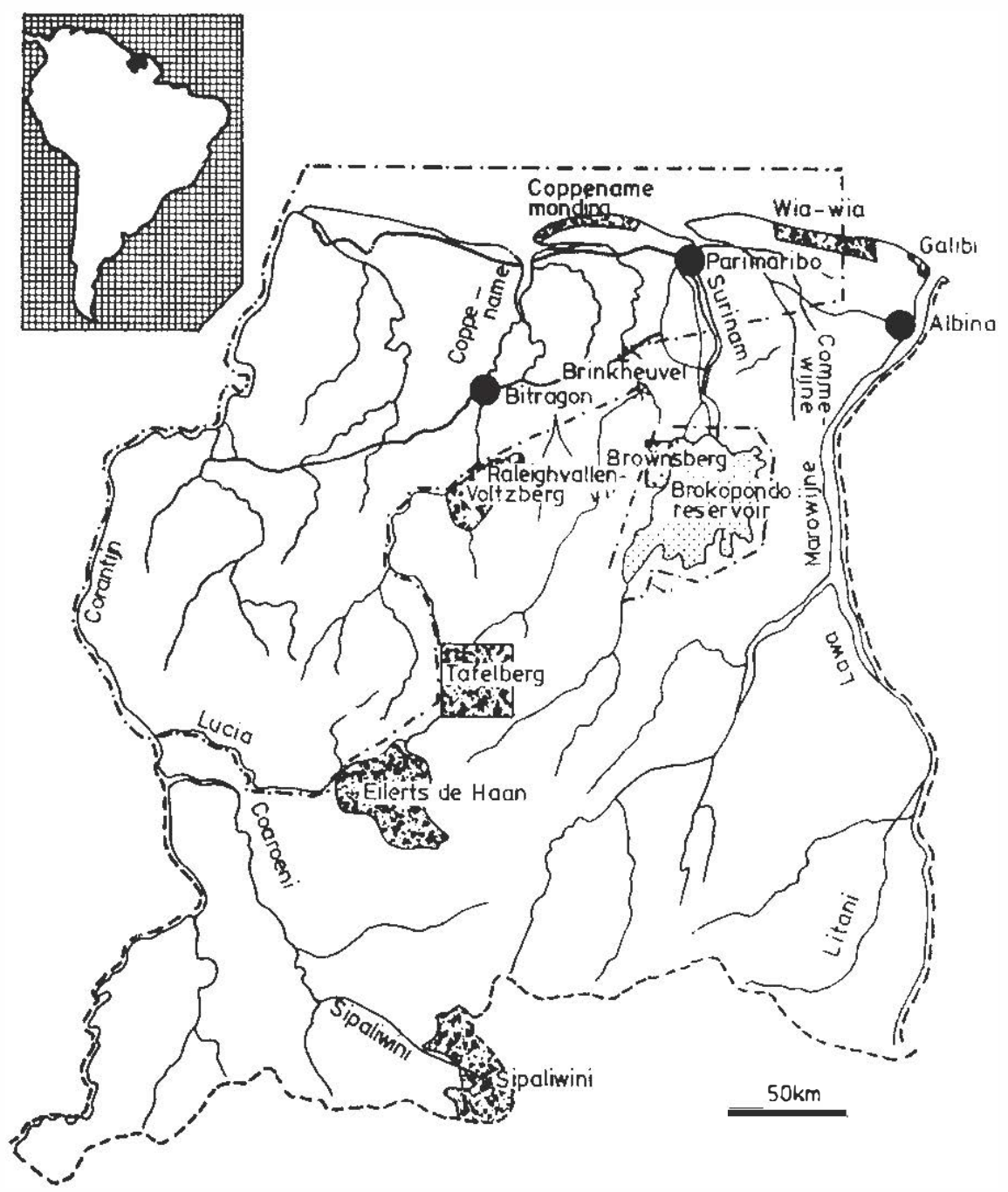

Figure 5 Map of Surinam showing the location of the Raleıghvallen Voltzberg Nature Rese't ve and the other eight protected areas in the country. 
High rain forest or high forest is found in areas not affected by seasonal flooding of rivers. The soil varies from loamy sand to clay, drainage is fair to good and litter decomposition is good. It is usually possible to distinguish three or four storeys in high forest. The up. per storey consists of emergent trees which can reach 40-50 m and occasionally higher (in Surinam only Cedrelinga cateniformis reaches $60 \mathrm{~m}$ ). Below the emergents is the canopy, which ranges from $15-30 \mathrm{~m}$ and can itself be divided into three categories, the upper part (25-30 m), the middle part (20-25 m) and the lower part (15-20 $\mathrm{m})$. The understory consists of slender trees up to about $15 \mathrm{~m}$, whereas saplings and undergrowth species make up the bottom $3 \mathrm{~m}$.

In general, the crowns of the emergent trees are more wide than deep and spread over the top of lower trees around them. No lianes connect them with the canopy crowns. The canopy crowns are almost as wide as they are deep and are connected by many lianes, whereas those of the understory vary from long and narrow to tapering. Many species of liane (some important families are Bignoniaceae, Celastraceae, Convolvulaceae, Ieguminosae, Menispermaceae and Moraceae) occur in high forest, but individuals of each species are usually widely dispersed. Epiphytes, including many Araceae and Orchidaceae, occur mainly in the crowns of trees. Stilt roots are rare, but buitresses are common. The herb layer is very open and consists mainly of broad-leaved forest grasses, small saprophytes and shrubby Rubiaceae Palms are common in high forest. Oenocarpus bacaba reaches the canopy, whereas Astrocaryum, Attalea and Bactris are found in the understory (Iindeman and Mooleneaar, 1959).

High forest is very rich in species and seldom shows any tendency to single-species dominance. The abundance of species makes it difficult to characterize high forest flor istically. In the preser:t study, the floristic composition of high forest and high mountain savanna forest in the Voltzberg region is partially analyzed by means of $1 / 2$ ha plots, in which about all trees and lianes representing potential food sources for Ateles are plotted (Appendix). The most important vegetational attributes measured are number and distribution of the component plants. The most decisive criterion in the evaluation of relative importance of a species in a given forest stand is the number of individuals. Success in the struggle for existence is 


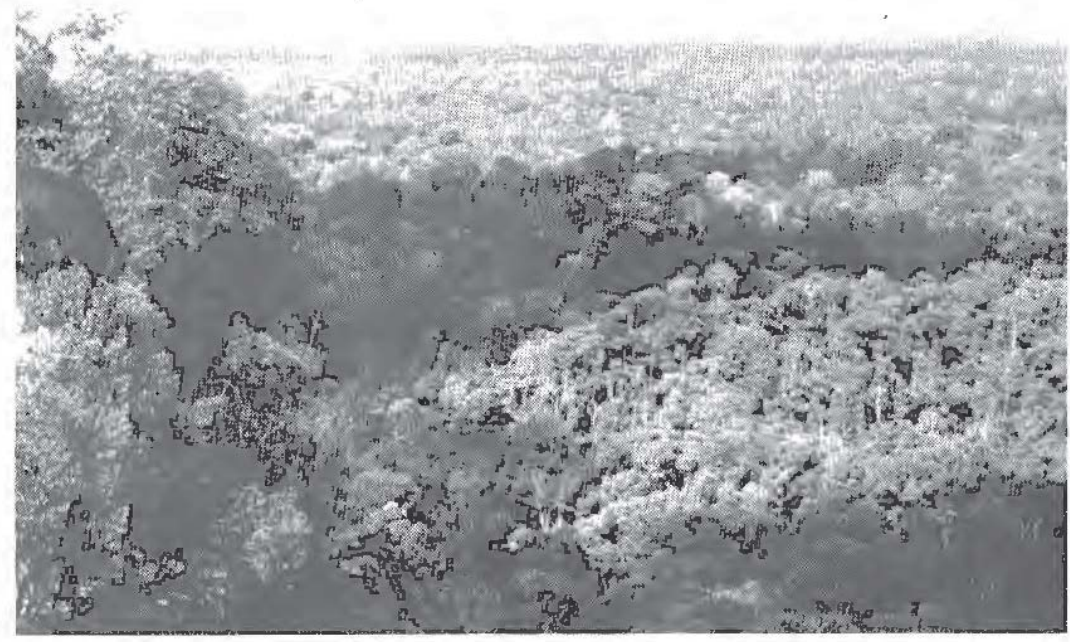

Figure 6 Scenic view looking south from the top of the Voltzberg, with part of the study area in the foreground. Note the other inselberg, the Van der Wijck top, at the horizon.

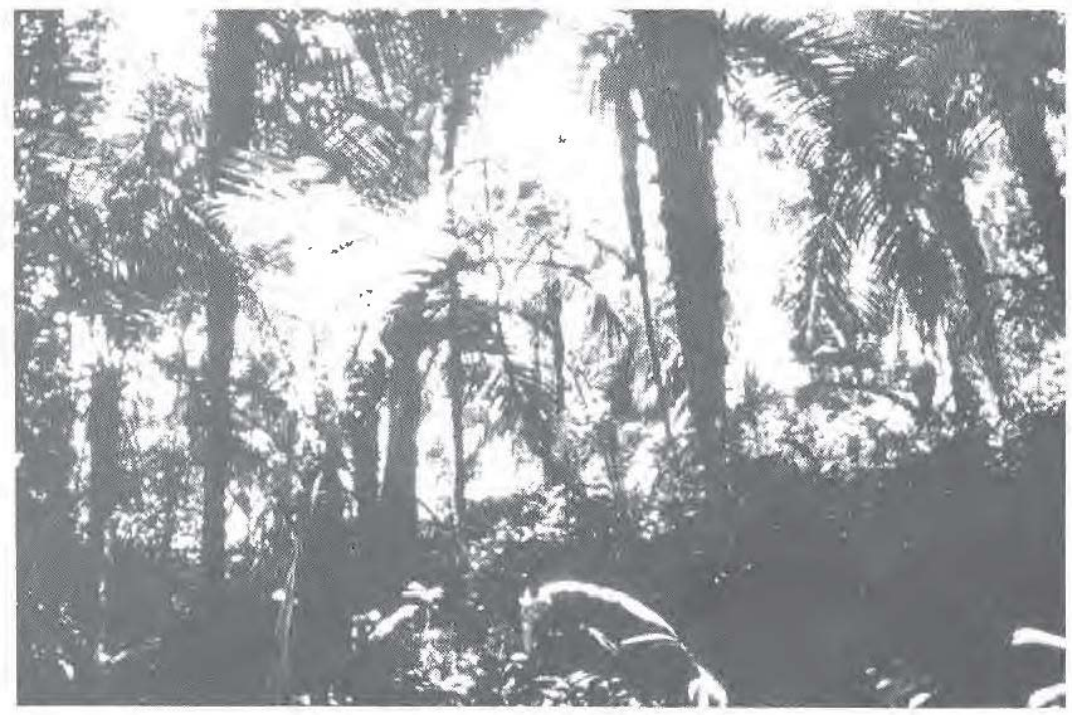

Figure 7 Understory and shrub layer of high forest in the Voltzberg study area, locally do. minated by the "boegroemaka" palm (Astrocaryum sciophilum) 
shown by reaching a reproductive state. The composition of the forest shows distinct variations from place to place. Some species show a wide ecological amplitude, others do not and are more local. Also the method of dispersal probably plays an important role. For example, seeds may be dispersed by wind, by water, endozoochorically, exochorically by rodents or autochorically. Figure 8 shows the distribution pattern of some species from high forest following diffferent dispersal strategies. It seems likely that species dispersed by wind and those dispersed endozoochorically will show a random distribution pattern (e.g., Vataireopsis speciosa and Clarisia racemosa, resp.) when it is considered that the entire seed shadow under and nearby the parent plant will be destroyed by terrestrial seed predators (e.g., bruchid weevils and rodents) which focus on these concentrations of seeds (pers. obs.). On the other hand, species dispersed by scatter-hoarding rodents may show a clumped distribution pattern (e.g., Eschweilera corrugata) since these animals do not carry seeds far away from the parent plant for storage. Seeds that escape from predation in this way and manage to grow success. fully will produce a non-random distribution pattem. This will also be the case in species which produce relatively small fruits containing many seedlets, such as berries. The seeds are mostly donnant, only genninating in a natural clearing caused by treefall. Germination is induced by strongly fluctuating soil temperature (as in $\mathrm{Ce}$. cropia latiloba (Holthuyzen and Boerbeem, 1982)

As demonstrated in figure 9, high forest has far more species producing edible fruit for monkeys and man than any other forest formation. Of a total of 486 species of fruit with an edible layer recorded for Surinam, 331 (68.1\%) are found in high forest. Consequently, high forest is the most important primate habitat in Surinam. All eight primate species occur in high forest and several (e.g., Ateles, Cebus nigrivittatus and Chiropotes) are largely restricted to this formation.

The high forest of the Voltzberg region seems to offer a somewhat poorer appearance than the lowland rain forest in its optimal form. In general, the lower part of the canopy and the undergrowth are poorer in species, perhaps due to the abundance of boegroemaka palms (Astrocaryum sciophilum) that locally form a fairly continnuos layer at about $8 \mathrm{~m}$ height (fig. 7). The ground flora is very 


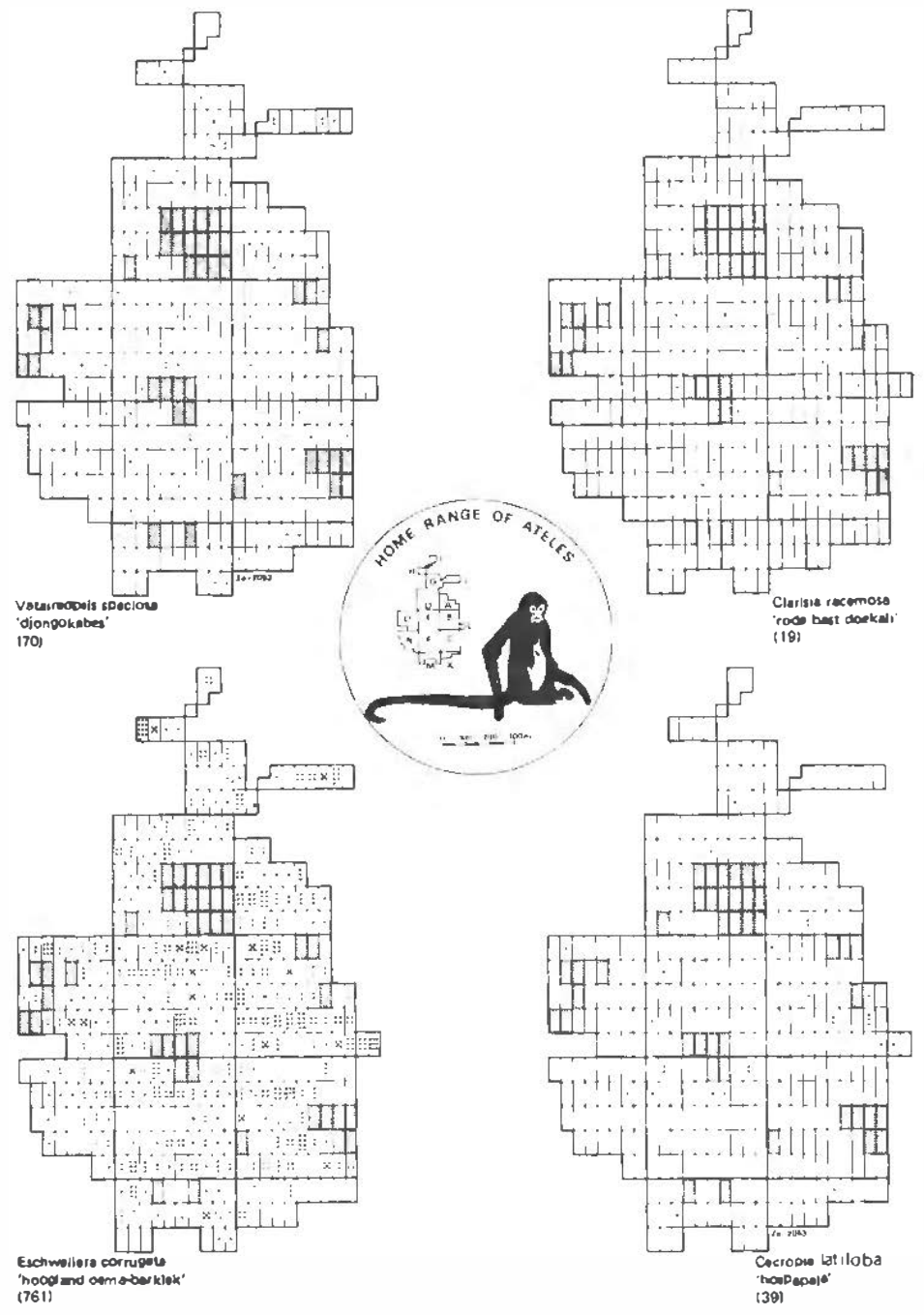

Figure 8 Distribution patterns of four tree species occurring in high forest in the Voltzberg region. The sampled area shown in the figure is part of the Voltzberg study area and is divided into $1 / 2$ ha plots. The shaded areas indicate open granite and liane forest. Vataireopsis speciosa, an emergent tree, has wind-dispersed winged fruits; Clarisia racemosa has nutritious fruits containing one large seed, that is dispersed only by spider monkeys; Eschweilera corrugata has woody operculate fruits containing several large edible seeds that are mainly eaten and stored by agoutis and acouchis, and Cocropia latiloba is a pioneer plant, growing in edge habitats and treefall-clearings, dispersed via dormant seeds by a number of opportunistic fruit-eaters including many birds. 
spare Young acaulescent buegroemaka palms locally dominate and, together with the old palms which have a well-developed trunk up to $5 \mathrm{~m}$ and sometimes even up to $12 \mathrm{~m}$, they effectively intercept the light. High density of this palm seems to be possible because of its dispersal by scatter-hoarding rodents, its extremely slow growth, and its broad fishtrap-like, heavily spined crown that catches falling leaves and continually produces a circular heap of humus around the base of the trunk.

The observer, once experienced in correctly cutting leaves off the spiny young boegroemaka palms, could walk very easily thorough this type of high forest, an important factor in tracking fast moving monkeys as Ateles. In addition, it makes it easy to find one's way back or to trace a route of monkeys previously followed. The silvery underside of cut palm leaves clearly marks the trail through the forest.

Low rain forest. This term is used to designate a type of high forest that does not exceed $20 \mathrm{~m}$ in height. It is far richer in lianes than neighboring high forest and has far fewer boegroemaka palms. It usually forms a transition between high forest and liane forest, and sometimes between mountain savanna forest and liane forest. It isn't shown on the vegetation map (fig. 17) as it usually covers only small strips along the margins of other forest types.

Riverbank high forest. Riverbank high forest is absent from most of the coastal region, but it is common in the interior. In areas where river banks are high and not affected by seasonal flooding of river margins, riverbank high forest may grow right up to the river's edge. The structure of this type of forest is very similar to that of inland high forest, but the composition is clearly different. A continuurn seems to exist; in the Raleighvallen area several indicator species for riverbank high forest could be determined, and these disappear about $700 \mathrm{~m}$ inland. The Voltzberg study area began almost two kilometers from the bank of the Coppename River and, therefore, this forest type was not present there.

\section{Mountain Savanna Forest}

Mountain savanna forest occurs on bauxite hills and low mountains where only a thin layer of soil covers the underlying rock. It is similar to white sand savanna forest in xeromorphy, thin-stemmed aspect, coriaceous structure of leaves and lack of clear differentia- 


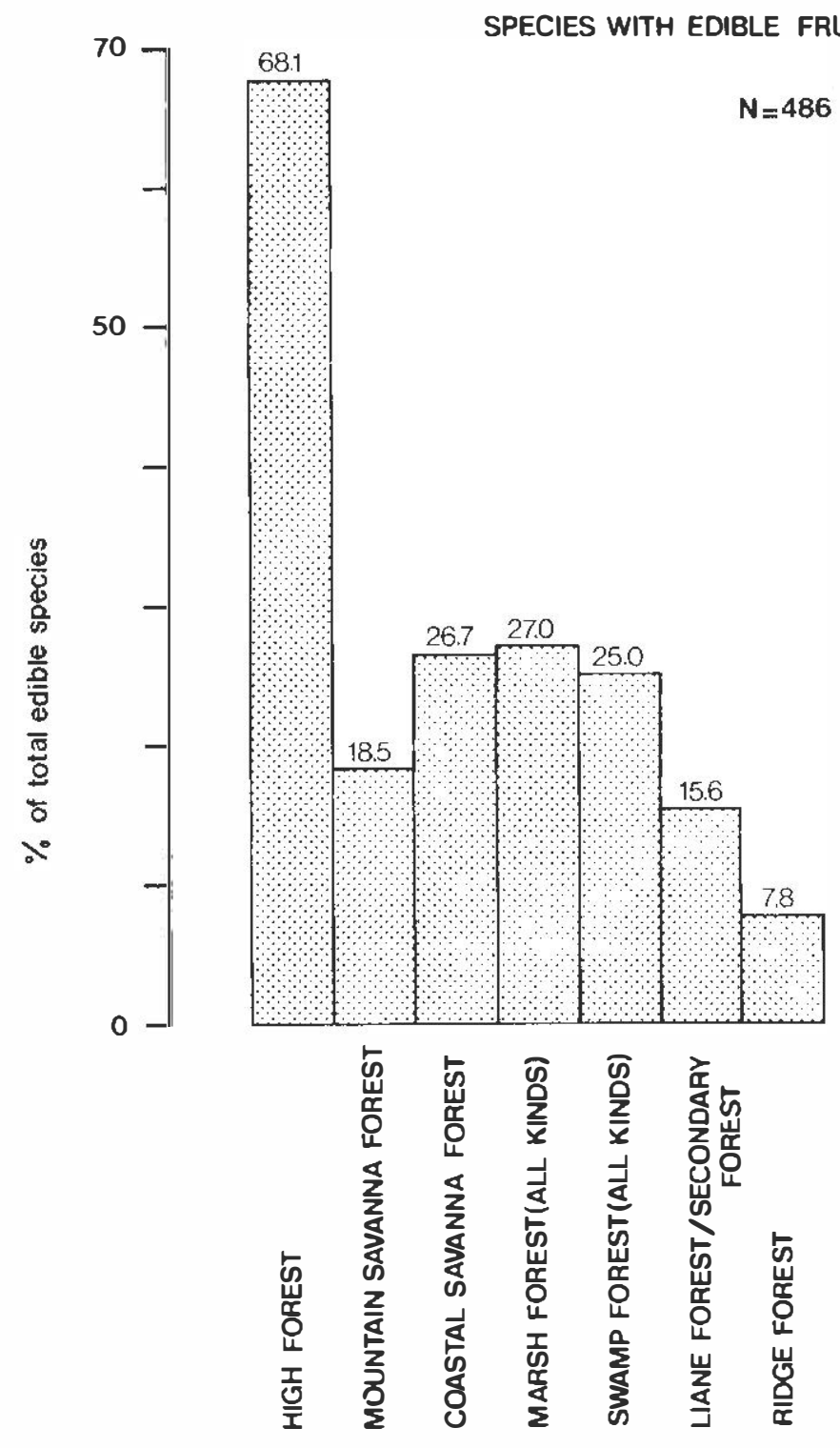

Figure 9 Woody plant species that produce fruit with an edible layer for monkeys and man and, exoed $2 \mathrm{~m}$ in beight in high tras and soveral cher trast types in Surinam. Not included are the families Araceae and Palmae or those species that produce edible seeds. High forest has far more kinds of edible fruit than any other formation. Data on edibility are taken from Van Roosmalen's study on Surinam fruits (1977). 
tion into storeys (it has a rather regular canopy of small crowns with few emergents), but it differs in floristic composition. Lianes are common, but not nearly as common as in fonnations such as liane forest. Understory palms such as boegroemaka are conspicuously absent. The undergrowth consists mainly of shrubs and treelets of the families Rubiaceae and Myrtaceae, and is easy to walk through. Visibility is much better than in high forest (fig. 10).

The dominant plant families in mountain savanna forest are Myrtaceae, Rubiaceae and Sapotaceae, which include several important fruit - producing trees for monkeys. In total, 90 species of tree and liane producing fruit with an edible layer have been recorded from mountain savanna forest (fig. 9).

A high mountain savanna forest fonnation, intermediate between typical mountain savanna forest and high rain forest, sometimes $\mathrm{oc}$ curs on more favorable parts of the bauxite plateaus and in granite areas such as those surrounding the Voltzberg and the Van Stockumberg in the upper Coppename region. Some of the dominant species in this forest fonnation are Ecclinusa guianensis, Guettarda acreana, Lafoensia pacari and Pteocarpus vs. santalinoides. At edges high mountain savanna forest one trequently finds important tood trees for monkeys such as Ceiba pentandra, Hymenaea courbaril and Spondias mombin.

During extremely dry seasons (e.g., 1976), which seem to occur irregularly at intervals of a few to many years, most of the trees from mountain savanna forest drop their leaves, giving the forest a dead appearance. In normal years only some of the trees seem to be deciduous

\section{Liane Forest}

Liane forest is a formation in areas with stony lateritic soils providing bad rooting conditions and poor foothold for trees. It is noteworthy for the absence of storeys. Tall trees do occur, but they are so widely separated from one another that no true canopy exists. The space between the trees is filled with dense tangles of lianes, vines and twiners that grow in abundance because of unrestricted exposure to sunlight (fig. 11). Although occasional trees in liane forest may reach $30 \mathrm{~m}$ or more, the liane tangle itself rarely exceeds $10-15 \mathrm{~m}$. 


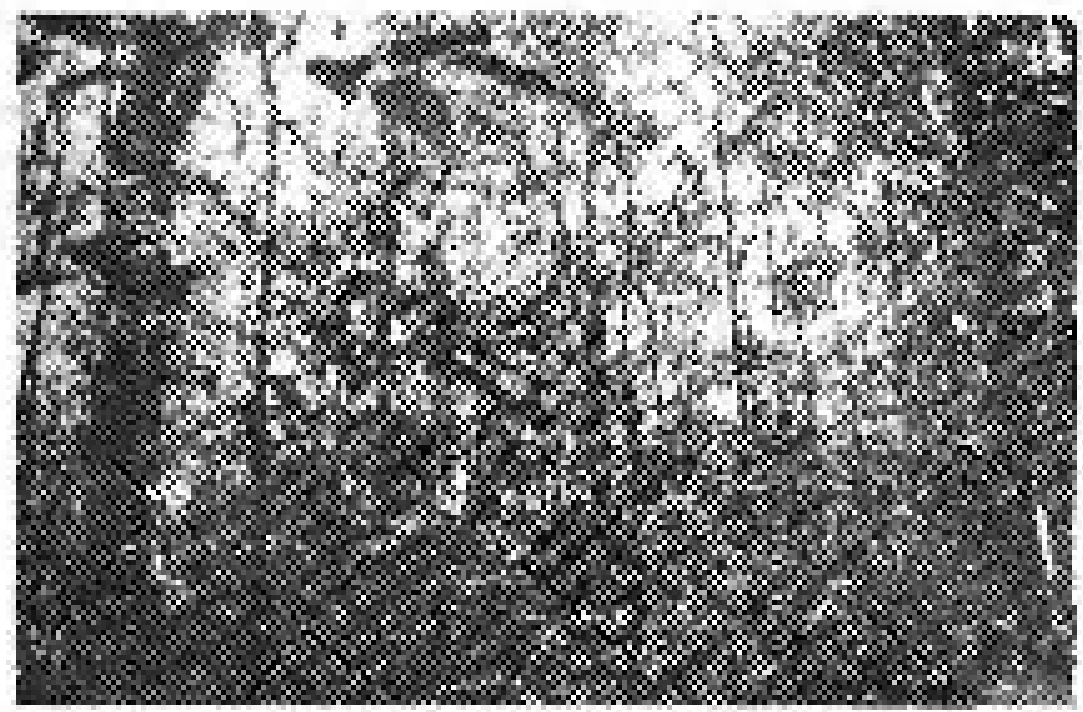

Figure 10 Mountain savanna forest in the Voltzberg study area. Note the thin, staky appea. rance of most of the trees and the lack of understory palms.

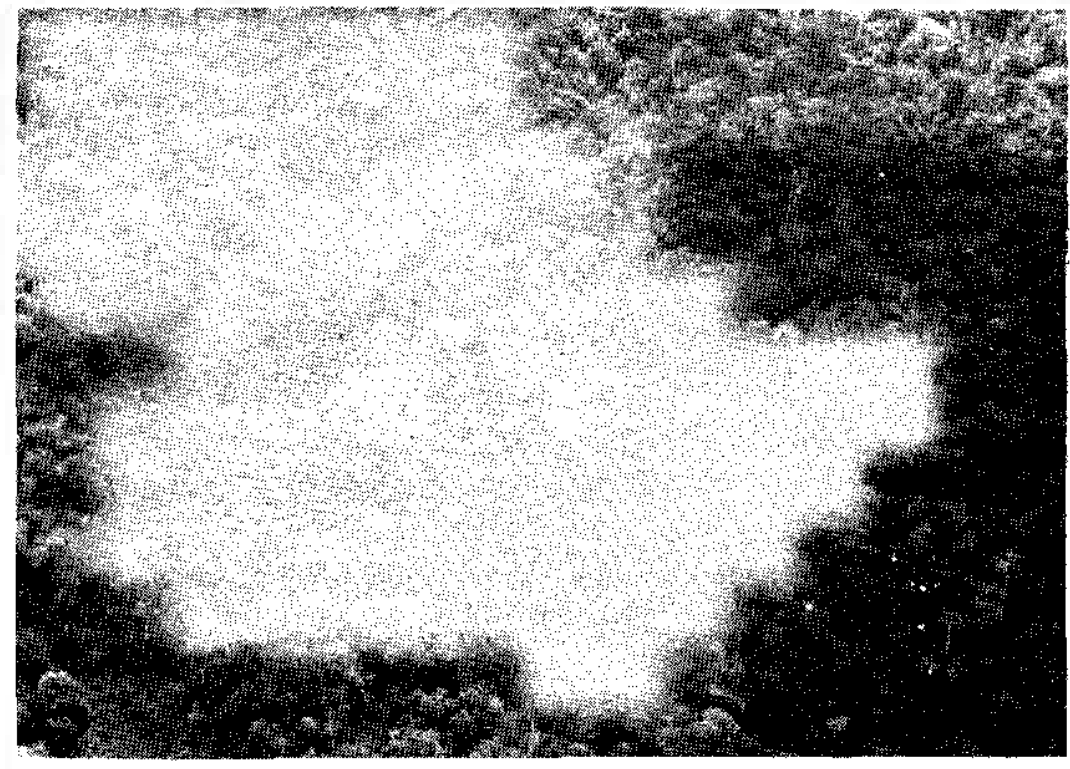

Figure 11 Aerial photograph of liane forest surrounded by high forest in the Raleighvallen - Voltzberg Nature Reserve. Note the isolated large-crowned emergents of the species Vitex stahelii. 
A large number of species are represented in liane forest, but most of them are of very low frequency. A total of 76 species producing fruit with an edible layer have been recorded from liane forest in Surinam. The dense liane tangles provide many microhabitats for insects and other arthropods, making them a fertile foraging ground for partly insectivorous monkeys (e.g., Saimiri and Cebus).

\subsubsection{WET FOREST TYPES}

\section{Swamp Forest}

Following Beard (1955), the term swamp forest is used for forest on soil which stays wet to damp throughout the year and as a result aeration of this soil is permanently impeded. Swamp-forest soils are actually under water most of the year and, if not inundated, at least remain damp during the peak of the dry season. The soil is usually composed of heavy clays and litter. The litter decomposes poorly because of bad aeration and accumulates to form a peat layer.

In the Raleighvallen-Voltzberg region only a single type of swamp forest is present along the small creeks and streams that flow almost all year round. Because of dominance of the pina palm, Euterpe oleracea, which locally forms pure stands, it is called pina swamp (fig. 12). Pina swamp forest reaches at least $20 \mathrm{~m}$ in height and, because of scattered tall trees, it cannot be differentiated clearly into storeys. The irregular canopy ranges from $18-30 \mathrm{~m}$ and is characterized by trees such as the buttressed Virola surinamensis and Pterocarpus officinalis, the stilt-rooted Symphonia globulifera, and Pachira insignis and Eperua falcata, all of which are food plants of Ateles. The most common tree is Euterpe oleracea itself, reaching the canopy. Another palm tree, Astrocaryum sciophilum, is common, especially in drier places. Visibility is good. The undergrowth is open, since it is formed by relatively few saplings of the above mentioned species and Euterpe oleracea itself. Locally, a dense herb layer can be present, which can hinder progress.

A total of 122 tree and liane species producing fruit with an edible layer have been recorded from the various kinds of swamp forest, but pina swamp forest alone is rather poor in edible species (fig. 9). 


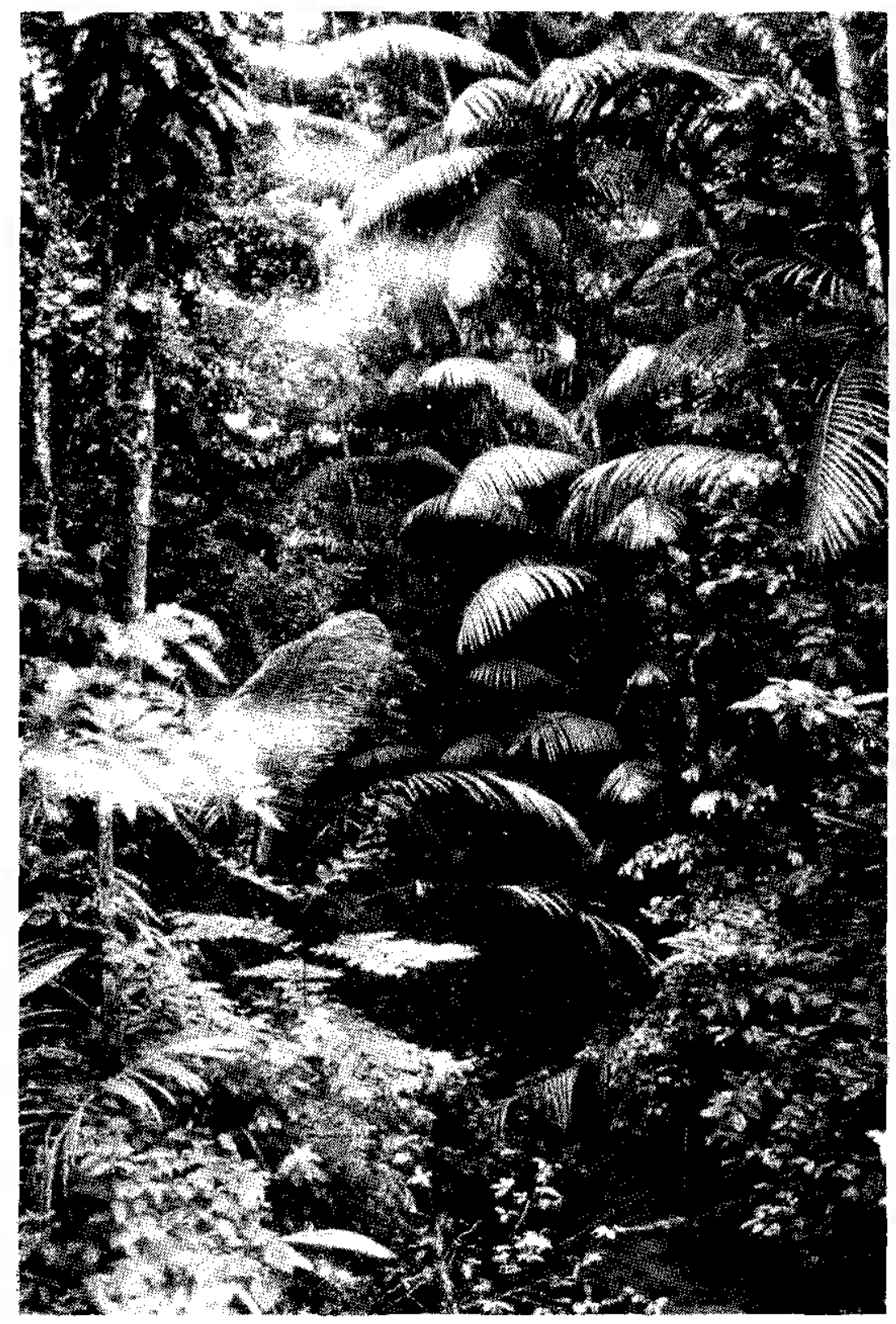

Figure 12 Pina swamp forest along a creek flowing through the Voltzberg study area. Note the abundance of the pina palm (Euterpe oleracea). 


\section{Marsh Forest (Seasonal Swamp Forest)}

Marsh forest or seasonal swamp forest in Beard's (1955) terminology is the term used for a number of two-storey forest types whose soils are periodically but not permanently inundated during the year. In drier parts of the year they lie above the ground-water table, making aeration possible.

Marsh forest is an important habitat for several monkey species.

The dense, liane-covered lower storeys along river margins provide excellent foraging grounds for the partly insectivorous species such as Saimiri sciureus. Marsh forest is rich in plant species, including many with seeds dispersed by wind or water. Of all tree and liane species producing fruit with an edible layer, $131(27 \%)$ are found in marsh forest. However, the only type of marsh forest occurring in the Raleighvallen-Voltzberg region is riverbank marsh forest. Riverbank marsh forest. This type of marsh forest occurs along river margins and is seasonally flooded by rising waters. The maripa palm, Attalea regia, can be regarded as a good indicator species for all types of marsh vegetation. This palm and several Bactris spp. can dominate locally to create palm forests'.

\subsubsection{XEROMORPHIC VEGETATION}

\section{Rocksavanna}

Rocksavanna is a rare vegetation type found in the Voltzberg region only on granite where the rock is bare or covered by shallow soil. It consists of bushes, terrestrial epiphytes, cacti and grasses. No palms occur, but some thin lianes and twiners are present. Maximal height is $5 \mathrm{~m}$. The vegetation is not closed. At edges of rocksavanna areas, an important edible-fruit producing tree, Spondias mombin, can be found (fig. 13) 


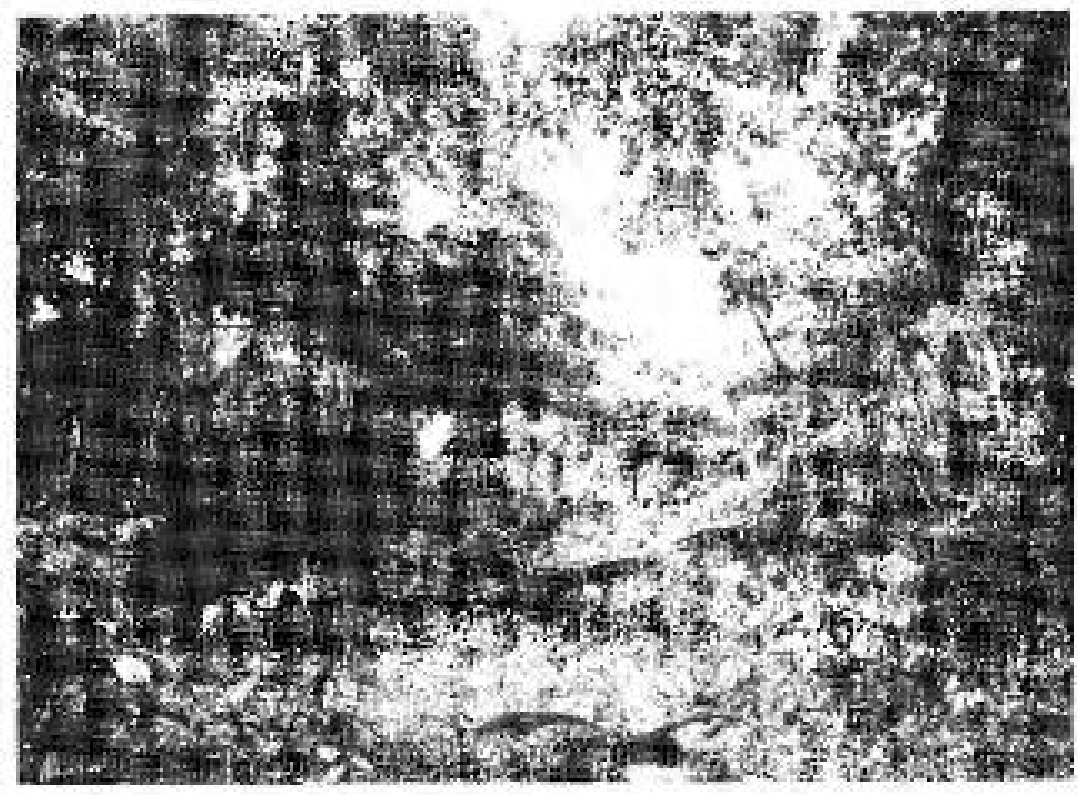

Figure 13 Transition from mountain savanna forest to rocksavanna. Important edible-fruit producing trees such as Spondiās mombin and Ecclinusa guianensis can be found at edges of rocksavanna. 


\section{METHODS}

\subsection{The Study Area}

The present study was conducted in the Raleighvallen-Voltzberg Nature Reserve, a protected area of 56,000 ha that was established in 1961 and is currently managed by STINASU; the Surinam Nature Conservation Foundation. It is located on the east bank of the Coppename River in central Surinam (fig. 5) and is bordered on the north by the Kwama Creek and on the south by the Tanjimama Creek (fig. 14). It includes Raleighvallen, the boulder-strewn rapids and falls (fig. 15) that mark the limit of navigability of the Coppename River, and several granitic 'inselbergs', most notably the 240 $m$ dome-shaped Voltzberg (fig. 16) and the Van Stockumberg. The headquarters of the Raleighvallen-Voltzberg Nature Reserve are on Foengoe Island, which is situated at the lower end of the Raleighvallen rapids. The island can be reached by plane or by boat. A three-hour trip by car followed by a three to five-hour trip by motorized dugout-canoe brings a visitor to Foengoe, situated about six kilometers from the Voltzberg. It has restricted facilities for tourists and researchers and it served as a main base during the present study.

In March 1976, together with the primatologist R.A. Mittermeier, a detailed reconnaissance was carried out and the borders of the Voltzberg Study Area were established. With the help of several Surinam field assistants a grid of main trails was cut at $500 \mathrm{~m}$ inter. vals, for a total of $15.5 \mathrm{~km}$ of trail. In the 130 ha area surrounding the camp and at the edge of a large granite plate, an additional $11.2 \mathrm{~km}$ of side trails were cut at $100 \mathrm{~m}$ intervals, parallel to the long axis of the study area. The finer grid was necessary in this area because it was the most important part of the spider monkey group home range.

The study area consisted of 10 blocks of 25 ha each, two incomplete blocks limited by the Voltzberg and together covering about $44 \mathrm{ha}$, and a final section of about 12 ha extending the study area to the start of the main tourist trail ascending the Voltzberg (fig. 14). When the surface of the two large, open-granite clearings in. the study area is subtracted, the total forested habitat was almost 
exactly 300 ha. This study area was used during the first year for gathering synecological data on all eight Surinam primates. In the meantime, a group of spider monkeys was selected and the boundaries of its home range determined very roughly. It soon became

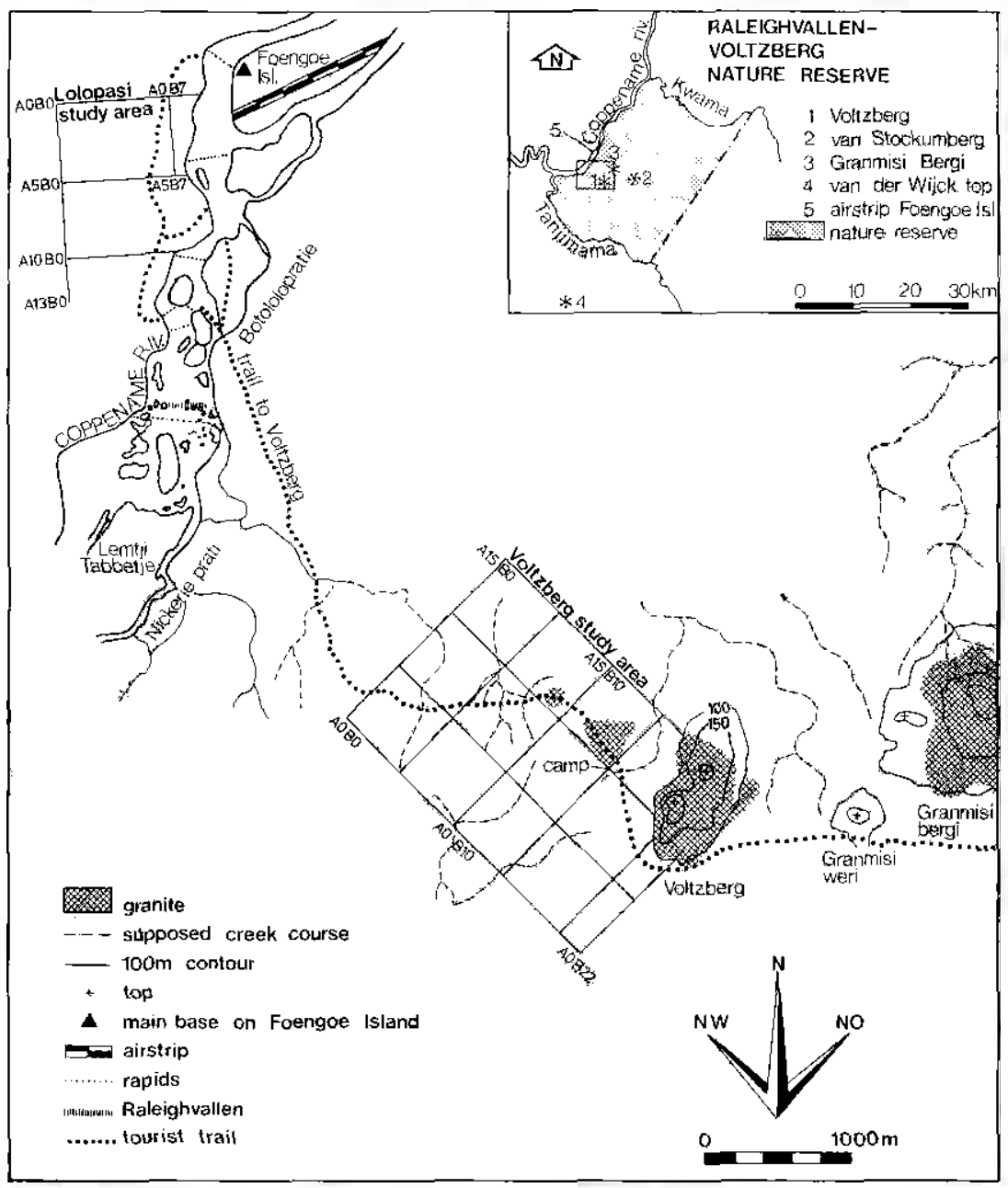

Figure 14 Map showing the location of both study areas in the Raleighvallen-Voltzberg $\mathrm{Na}$ ture Reserve. The trail system of the Lolopasi study area was not completed since the group of spider monkeys living in the area was too difficult to follow. No progress was made in habituating them. This may have been due to light hunting pressure in the recent past. The main study area at the foot of the Voltzberg dome bas only major trails at $500 \mathrm{~m}$ intervals indicated. 


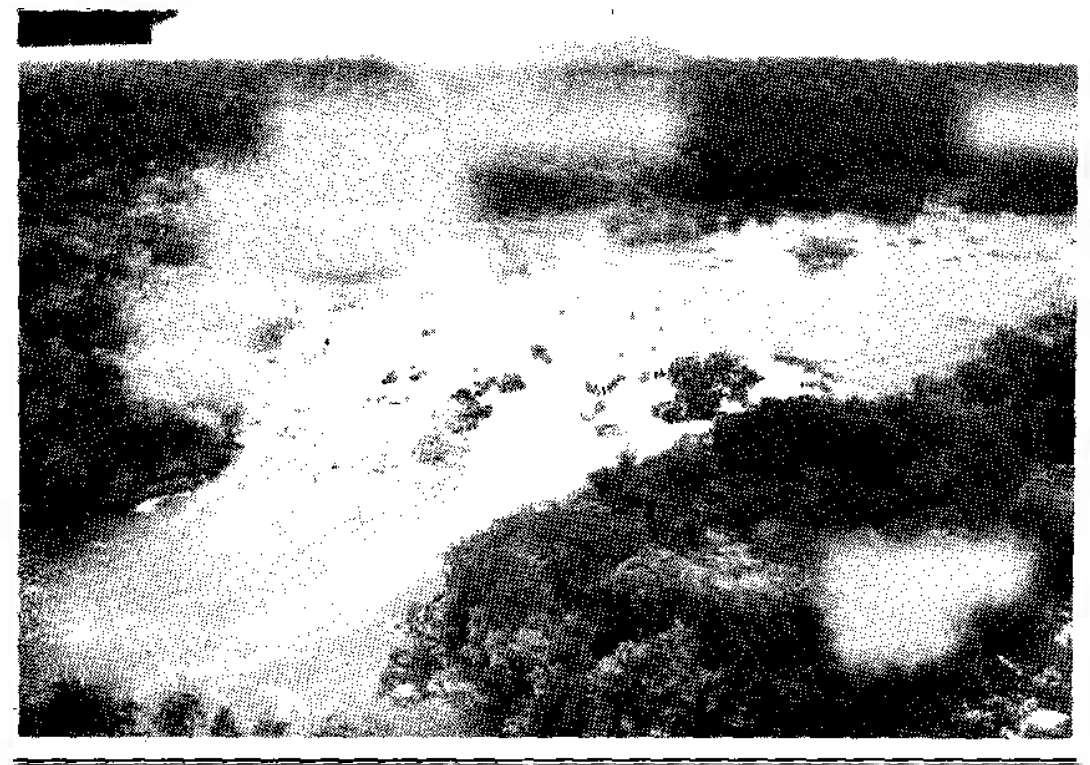

Figure 15 The $240 \mathrm{~m}$ Voltzberg (right in the background) and the $360 \mathrm{~m}$ Van Stockumberg (left in the background) seen from Raleighvallen rapids in the Coppename River about two kilometers upstream from Foengoe island.

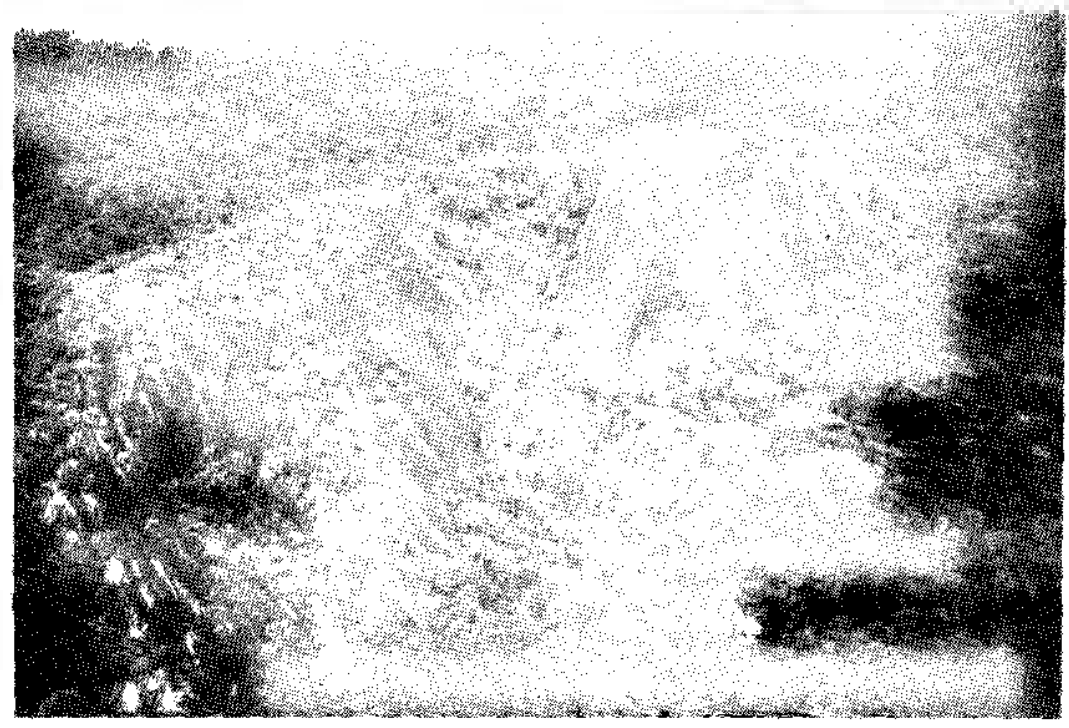

Figure 16 Aerial photograph of the Voltzberg. The forest in the foreground is part of the study area. 
evident that the original study area had to be extended approximately 15 ha to the northeast and 25 ha to the southwest in order to cover the entire range of the group (fig. 17).

All $100 \mathrm{~m}$ and $500 \mathrm{~m}$ trails were provided with red-painted sticks at $50 \mathrm{~m}$ intervals. Each of these had aluminium tags showing their coordinates in relation to an $\mathrm{A}$-axis directed $\mathrm{NE}$ and a $\mathrm{B}$-axis directed SE from a point of origin at the western corner of the study area. After trail-cutting, a vegetation map was made by first pacing off vegetation boundaries along all the trails and marking them on the map. Then, each vegetation boundary was traced by compass and mapped. Obviously, vegetation mapping is most accurate where the trail system is most extensive, namely in the main part of the spider monkey home range.

The forest in the study area can be divided into four majo: types (fig. 17). High forest predominates, followed by liane forest and mountain savanna forest. Pina swamp forest grows along the small creeks that flow through the area. Low forest is present as a transitional type but hasn't been mapped, as it usually occurs in narrow strips along the borders of liane forest. The Voltzberg study area has a greater diversity of forest types and edge habitats than would usually be expected in a tract of similar size in the interior. This is probably'caused by the abundant granite outcroppings that do not provide sufficient support for most tall forest trees, but do permit the growth of comparatively rare formations such as mountain savanna forest and liane forest.

Since spider monkeys never enter low forest or liane forest, these formations, together with open granite and rocksavanna, act as natural boundaries. As shown in figure 17, large liane forest complexes and the Voltzberg itself form a considerable part of the boundaries of the spider monkey home range, giving it a peninsula-like appearance.

Other animals present. All eight primate species were well represented. Besides Ateles paniscus, one could regularly observe Saguinus midas, Saimiri sciureus, Cebus apella, Cebus nigrivittatus, Chiropotes satanas, Pithecia pithecia and Alouatta seniculus. In addition to these primate species, a number of other mammals, as well as birds and reptiles, inhabit the Voltzberg study area. Larger rodents such as the agouti (Dasyprocta leporina) and the acouchi (Myo- 


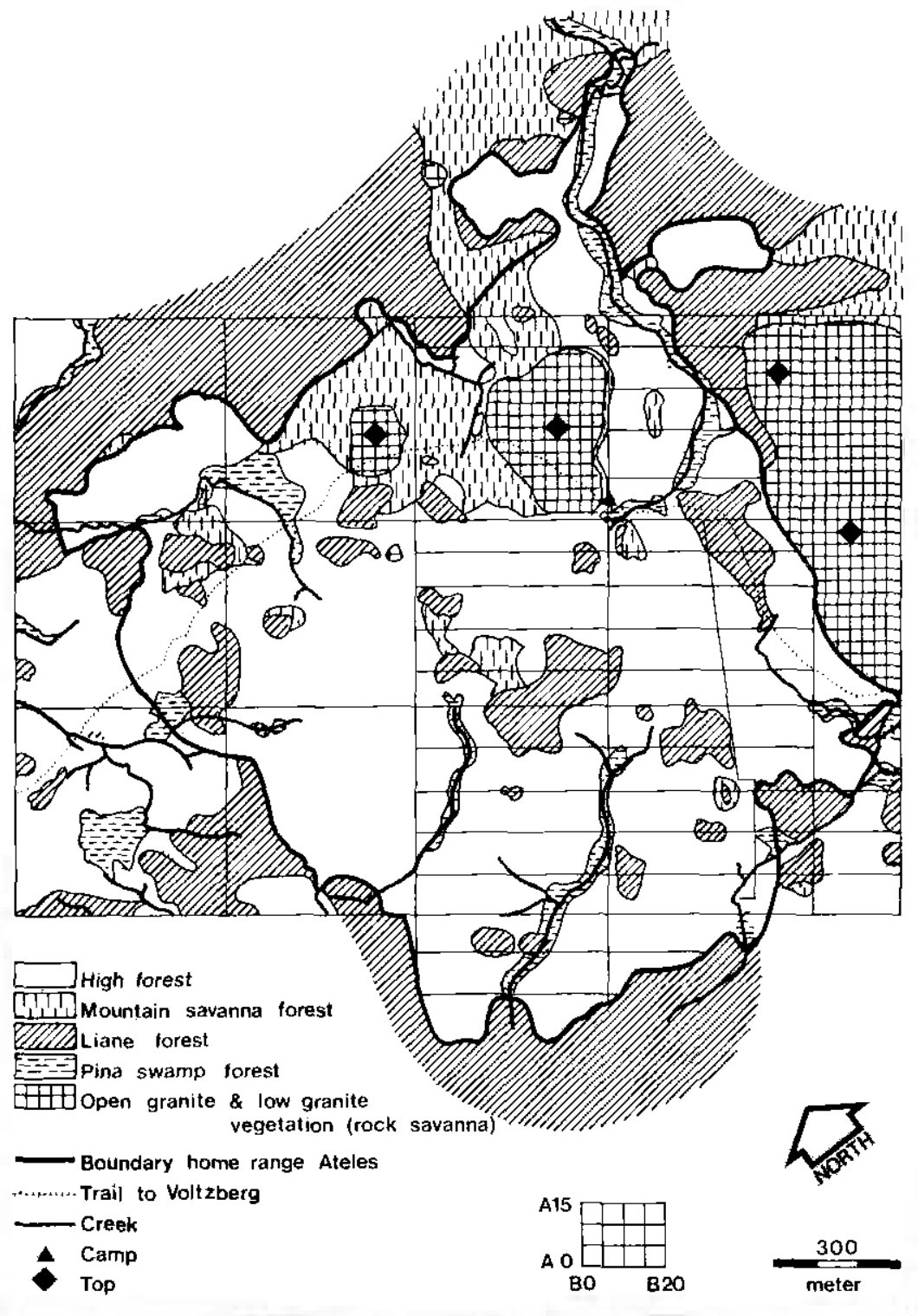

Figure $17 \mathrm{Map}$ of the Voltzberg study area showing distribution of forest types and trail system. The areas of open granite, low granite vegetation ("rock savanna") and liane forest were not used by the spider monkeys. The large area of open granite at the right is the Voltzberg dome with its two tops. The camp is situated at the edge of the larger of the two granite plates. The perimeter of the spider monkey group range is marked by a heavy line. 
procta exilis) are commom. Two kinds of 'squirreel(Sciurillus pusillus, Sciurus aestuans) and the prehensile-tailed porcupine (Coendou prehensilis) also occur, but are rarely seen. The camivores are represented by one or two jaguars (Panthera onca), several ocelots (Felis pardalis) and a number of tayras (Eira barbara). Edentates include the giant armadillo, (Priodontes giganteus), the nine-banded armadillo (Dasypus novemcinctus), the tamandua (Tamandua longicaudata), the giant anteater (Myrmecophaga tridactyla), and two kinds of sloth (Choloepus didactylus, Bradypus tridactylus). The common opossum (Didelphis marsupialis) and several smaller marsupials (e.g., Marmosa spp.) are present. Ungulates are represented by several collared peccaries (Tayassu tajacu) and at least one tapir (Tapirus terrestris).

Many species of bird live in the study area and a comprehensive list is given in Mittermeier and Milton (1976) and in the field checklist of the Birds of the Guianas (Davis, 1966). The most conspicuous are large species such as the black curassow (Crax alector), the marail guan (Penelope marail), the gray-winged trumpeter (Psophia crepitans) and the tinamous (Tinamus spp.). Cocks-of-therock (Rupicola rupicola) are surprisingly abundant. An important lek area and many nesting sites were found within and nearby the study area. In the canopy are scarlet macaws (Ara macao), toucans (Ramphastos spp.), aracaris (Pteroglossus spp.) and a variety of parrots, especially red fan parrots ((Deroptyus accipitrinus), orange-winged parrots (Amazona amazonica) and mealy parrots (Amazona farinosa). A number of raptors, including the harpy eagle (Harpia harpyja), occasionally visit the study area as well.

Frogs, lizards and snakes are common, and the turtles are represented by two abundant tortoises (Geochelone carbonaria and G. denticulata).

Insect life is varied and abundant, but mosquitos are rare. Insect discomfort usually is caused by chiggers and a number of stinging and biting ants.

\subsection{Data Collection}

2.2.1. Habituation of spider monkeys. During the first year, when the synecological part of the study took place, a group of spider monkeys was selected for the detailed autecological study that was 
planned for the second year (May 1977 - May 1978). Since the area had been undisturbed for at least several decades, the animals were not particularly shy. From time to time, tourist groups walk along the main trail to the Voltzberg thorough part of the group's range. Therefore it is likely that each member of the Ateles group saw people before this study began. In any case, the animals in the groups, except for one old male, no longer responded to people with the screaming - branchshaking and dropping-defecating display typical of Ateles that have had no contact with humans. They also didn't flee at the first glimpse of humans as do spider monkeys where they are hunted.

While studyings synecology of the eight Surinam primate species during the first year, many census walks were made of the entire study area. Data were collected while looking for 'target' species or tracking them. During this period, many contacts were made with all members of the spider monkey study group and occasionally with members of two other groups that touched the study area. Every time spider monkeys were located, first sighting data were collected for synecological purposes and the animals were followed for as long as possible. When the animals were lost, the observer returned to the trail and again used the trail system to search for other monkeys. The cutting of palm leaves, saplings and lianes seemed to disturb the monkeys most, but after a while the observer got very experienced in zigzagging through the shrub layer with minimal cutting.

From February through April 1977, attemps were made to follow spider monkey subgroups as long as possible. After many attempts, and aided by the observer's growing experience in walking freely through the forest while keeping visual and especially auditory contact, it became possible by the beginning of April 1977 to track spider monkeys day after day without noticeably disturbing either their ranging behavior or activity patterns. Working alone seemed to be essential for this. When accompanied by one or more observers, it was hard to maintain contact and the monkeys' behavior seemed to be influenced significantly.

2.2.2. The synecological part. The synecological study in the Voltzberg study area was initiated in March, 1976. Synecological data were gathered on all eight primate species for 10-15 days each 
month until April, 1977. A total of 132 field days were spent studying synecology only. During the first phase and later on, my colleague R.A. Mittermeier also collected data on synecology. We gathered data during 12 census walks of the entire study area, spread over a year, and while while looking for and tracking 'target' species selected for a given day. The following kinds of information were recorded every time a group was encountered: time, location in the study area, forest type, edge or non-edge habitat, activity (e.g., resting, traveling, feeding on plant food, foraging for or feeding on insects), height and level in the forest, how located (e.g., spontaneously seen, heard vocalizing, heard crashing, heard dropping fruit or faeces), path-animal distance and observer-animal distance (both for census purposes), group size and, if possible, group composition and direction of travel. All data on preferences for different forest types, vertical stratification, activity and location of the group were based on first sightings only, in order to avoid possible bias caused by the presence of the observer. When first sighted, the visible members of a subgroup were usually all engaged in the same activity, at the same level and in the same forest type, especially in the case of spider monkeys. In order to avoid confusion, first-sighting data were based on the first individual actually seen, which is essentially an instantaneous focal-animal sample.

A sighting was considered an edge when the first animal seen was within $20 \mathrm{~m}$ of a clearing or another forest type. Feeding on plant tood was considered the activity when the tirst animal seen was eating fruits, leaves, flowers or vegetable matter. Foraging for or feeding on insects was considered the activity when the first animal seen was eating or actively searching for insects or other animal life.

For stratification, observations the forest was divided into six levels : shrub layer $(0-3 \mathrm{~m})$, understory $(3-15 \mathrm{~m})$, lower part of the canopy (15-20 m), middle part of the canopy (20-25 m), upper part of the canopy $(25.30 \mathrm{~m})$, and emergents $(30-60 \mathrm{~m})$. It was usually impossible to estimate the height of emergents accurately because of intervening vegetation and the limitations of the clinometer. However, it was usually quite easy to determine whether or not a tree was an emergent simply by its relationship to surrounding trees.

Location within the study area was determined by using the markers situated at 50 intervals along the trails. Measuring the dis- 
tance from a particular tree to the nearest marker gave quite accurate coordinates for each sighting.

Synecological data continued to be gathered during the second part of the field study on spider monkey autecology. At this time Ateles became the 'target' species, and synecological data were only collected while searching for spider monkeys or when encountering other species while tracking spider monkeys.

2.2.3. The autecological part. The study on the autecology of spider monkeys in a strict sense was started in the Voltzberg study area at the end of April 1977, and lasted until the beginning of May 1978. For each month of attempt this full year an attemp was made to observe spider monkeys for seven full days, but in several months only five or six days were possible. A total of 135 days was spent in the field during this period, resulting in over 865 hours of observing only spider monkeys. Usually 10-14 days had to be spent each month in the study area, divided into two sessions of 5-7 days each. As a rule, the first and the third week of each month were spent in the study area, but sometimes the second and the fourth week were chosen.

In natural habitats, spider monkeys are usually observed in small subgroups of variable size and composition. The subgroups withir a group interact peacefully with one another, but groups are separated from one another by agonistic interactions, particularly between the males who appeared to act territorially and to respect quite clearcut boundaries. In order to spot a 'target' subgroup at the start of a session, several methods could be used. On returning to the study area after an absence of 7-10 days the situation had always drastically changed. Changes in the fruit-producing plant species and also in individual plants usually resulted in completely different foraging routes for the monkeys. A fresh start had to be made every time. Therefore, at the start, part of trail system was covered until a subgroup was encountered. The searching could last for hours or sometimes for as much as a full day. A long call by a male spider monkey, which was somentimes performed in response to imitation calls, helped in saving time. The calling male or "his" subgroup could then be found using a compass, or at least efforts could be concentrated on a more defined area. In case of prolonged lack of success, the Voltzberg could be climbed in the late afternoon in the 
hope of seeing spider monkeys entering one of the huge, usually leafless or early flushing sleeping trees. If so, the tree was located betore returning to camp and the next day an attempt was made to be present at the foot of this tree at $5.45 \mathrm{~h}$. It was then necessary to remain continuously with the animals until evening, between 17.00 $-18.30 \mathrm{~h}$, when they entered another sleeping tree. If contact with a subgroup was lost, it was necessary to return to the nearest trail and try to find this or another subgroup as soon as possible.

The following data were collected while tracking spider monkey subgroups. At five-minute intervals the composition of the subgroup was recorded, the activity of all members of the subgroup, and the stratum and forest type occupied. The coordinates of every trail crossing were recorded by pacing the distance to the nearest marker. When the animals were feeding complete samples (e.g., infructescences, inflorescences, leaves and/or bark) were collected whenever possible. Usually several visits to a particular food plant were needed, as spider monkeys generally show a very economic use of food resources. When the monkeys didn't drop a complete sample of the food plant, a collection was made as scon as possible by a native tree climber from the Surinam Forest Service. Each food plant actually used by the monkeys was marked with a numbered aluminium tag and a piece of red.fluorescent plastic flagging tape, nailed on just after the monkey(s) left the tree. Using a water-resistant pencil, date and monkey species were written on the plastic tape and, later, the coordinates of the food plant were determined. Food samples were collected in plastic bags sealed by a piece of plastic tape on which time and tag number were written. Back in the Voltzberg camp, a picture of all items eaten that day was taken with a Nikkormat EL camera, using a $52 \mathrm{~mm}$ macro lens. The samples were preserved by adding some formaldehyde ( $2 \%$ sulotion) and were then labelled. Upon returning to the base camp on Foengoe Island, all samples were identified if possible, using the book on the fruits of Surinam (Van Roosmalen, 1977) and monographs of certain plant tamilies and genera (Berg, 1975; Wessels-Boer, 1965). After making a drawing of each new food item, including cross-sections and other details, all samples were put in jars, labelled and stored.

Faeces were also collected and preserved. Spider monkey faeces usually consisted mainly of undisgested and unharmed seeds 
and stones (seeds protected by a hard endocarp), especially during the wet seasons. Consequently, the faeces were not compact and when they fell to the forest floor the seeds dispersed over quite a wide area. If this happened, as many of the seeds and/or stones as possible were collected.

Data on phenology of trees and lianes of many species, especially those used by monkeys, were gathered in the following manner. While searching for or tracking spider monkey subgroups, the observer visited almost every part of the groupu's range over the course of a few days. During these walks throughout the year, the presence of fresh flowers, fruits or fruit-parts on the forest floor was noted, and new species were collected and identified. In this way, timing and length of flowering and fruiting periods were determined for many plants, not only on a species but also on a individual level.

In September 1977, together with a native tree specialist and an assistant, a marking program was initiated, which lasted about seven months. By this time, almost all important food species of Ateles had been determined, and many of them had already been collected during the synecological part of the study. A list of 120 important species, including food trees, easily recognizable lianes and some sleeping trees, was compiled using vernacular names. When a species was not known to the tree specialist, samples from several individuals were shown to him so that he could learn its special properties such as type of habit, colour, taste and texture of baxk and/or wood, presence of latex, type of leaves, etc. Each species was given a code number. The most important part of the spider monkey group range, approximately 205 ha, was inventoried. For this purpose, new transects were cut at $50 \mathrm{~m}$ intervals, perpendicular to the $100 \mathrm{~m}$ trails, resulting in about 370 rectangular blocks of half a hectare each (fig. 8). All individual trees and lianes belonging to the listed species were marked with an aluminium tag different from the kind used to mark trees which the spider monkeys were actually seen feeding. In this way, block after block was inventoried and the location of each numbered plant was approximately determined by its place in a certain half-hectare block. Trees below $12 \mathrm{~m}$ were not included, as this was determined to be the lower limit of the spider monkey vertical range. For the purpose of 
this study, tagging and plotting was restricted to those individual plants that had obviously reached their own preferred stratum and this, in general, implies that they would flower and truit. Plants which provided the monkeys with only edible leaves were plotted when they reached $12 \mathrm{~m}$ or more, regardless of whether they had reached their preferred stratum.

A total of about 10,000 trees and lianes were marked and plotted in special maps in order to determine their density and pattem of distribution (Appendix). In this way most of the trees and lianes, which had been marked when spider monkeys fed on them, were marked again, thus giving a double check of identification and location. Also, this provided an idea of the reliability of the tree specialist, which turned out to be high.

For measurement of dietary composition, the frequency with which different foods were chosen was used. If a subgroup or a solitary ranging animal was seen feeding on a single food plant, one observation was scored. If a subgroup or individual moved to another tree of the same species, another observation was scored. If a subgroup or individual moved to a different food plant species, one observation was scored for that species. If identifiable seeds or stones of a given species, which had not been seen being eaten that day, were found in the faeces of a monkey, one observation was scored for that species. This method may seem to be somewhat crude, but it a gave a good estimate of the relative importance of different food plant species and families in spider monkey diet. This method is preferred to determining the total feeding time for each species, because feeding rate can vary widely between foods. Some fruits are swallowed whole, others are woody and indehiscent and have to be opened first. Some fruits contain a lot of nutritions pulp and comparatively small seeds or stones; others produce very little pulp or are eaten only for a minuscule aril. Measurement of the proportion of feeding time spent on different foods has also the disadvantage that analysis of faecal samples cannot be taken into account. Faecal samples can give much information, particularly for spider monkeys, since the animals swallow and do not digest seeds and stones in $93.5 \%$ of all fruit-feeding observations. The method of quantification used in this study produces some bias towards foods that are eaten regularly but in small amounts, and towards plant species that 
produce several edible items at the same time. Both features, however, are rare in the case of spider monkeys and consequently this bias is of little importance. A relatively more important bias may be that produced towards species that grow at high densities. For example, Virola melinonii (Myristicaceae) can be overestimated in this method because it is found locally in great abundance. When feeding on a particular tree of this species, the monkeys will also visit nearby trees, each of which provides only a small crop of truit. If these trees were more widely dispersed, they would probably be ignored because of the small energy yield per tree.

\subsection{The General Survey}

Some other localities were investigated during the general survey, and these added data to the distribution map the habitat table for Ateles.

\subsubsection{Lolopasi, west bank Coppename River, Raleighvallen-} Voltzberg Nature Reserve. Lolopasi is located directly across the Coppename River from Foengoe Island, site of the headquarters of Raleighvallen-Voltzberg Nature Reserve. A trail of $2.9 \mathrm{~km}$ has been cut through the forest to enable visitors to reach the Moedervallen, largest of the Raleighvallen rapids. During the first part of this study, additional trails at $500 \mathrm{~m}$ intervals were cut to the west, roughly perpendicular to the riverbank and $600-1,000 \mathrm{~m}$ inland (fig. 14). The trail system has not been finished, but a total of $7 \mathrm{~km}$ of trail were cut before the area was abandoned as a study site.

The Lolopasi area consists almost entirely of riverbank high forest and high forest, except for a narrow strip of marsh forest close to the riverbank. Pina swamp forest and some liane forest are present, but no mountain savanna forest occurs.

All eight Surinam monkey species were encountered in the area, but some of them, particularly Ateles, were very shy, probably because of recent hunting. It was impossible to keep track of spider monkeys for more than half an hour. No progress could be made in the habituation of the spider monkeys, but a rough idea of the population size and some dietary data were obtained.

2.3.2 Kayser Mountains. The Kayser Mountains are located in the interior, in southewestern Surinam. The Surinam Geological Servi- 
ce opened a large area for survey work by cutting transects. A twoweek survey of monkeys was carried out there in September 1976, using a trail system of about $40 \mathrm{~km}$. The area was undisturbed except for some hunting by the workers. Since certain preferred game species, such as black curassows (Crax alector) and peccaries ( $T a$. yassu tajacu and Tayassu pecari) occurred at high densities, these people did not hunt monkeys. The spider monkeys were not at all shy and reacted with typical first contact displays. The population density of Ateles and several other monkey species was determined to be somewhat higher than in the Voltzberg area. Less severe food scarcity in the long dry season seemed to be the main reason for this difference.

The vegetation is primarily high forest and mountain savanna forest, with some pina swmp forest in low-lying areas. Above $400 \mathrm{~m}$, the boegroemaka palm (Astrocaryum sciophiloum) of the under-growth and shrub layer of high forest is replaced by the bergimaripa palm (Atialea speciosa), which reaches the canopy (15-18 m).

Additional data on diet, subgroup composition and habitat preferences of Ateles were collected, as were synecological data on all monkey species present.

2.3.3. Other parts of Surinam. Locality data for the distribution map (fig. 1) were taken from museum specimens of Surinam monkeys, from field observations by R.A. Mittermeier, and from reports by several other naturalists well-acquainted with the monkeys. 


\section{HABITAT PREFERENCES}

\subsection{Preferences for Different Forest Types}

Where little or no hunting has taken place, Ateles p. paniscus can be quite abundant in tracts of high forest. It also occurs in riverbank high forest but this habitat has often been subjected is severe hunting by Bushnegroes and/or Amerindians been subjected to. Even in uninhabited stretches of riverbank high forest, there has usually been at least some hunting by occasional field crews from the Meteorological, Geological and/or Mining Services. I never observed spider monkeys right at the river's edge. The reasons may be unpleasant hunting experience in the past, tendency of the monkeys to avoid edge hanitats, and/or lack of preferred food plant species at the river margins. Aside from the preferred habitats, Ateles was seen in Surinam only once in marsh forest, once in high savanna forest growing on the Coesewijne formation and three times in Eperua falcata savanna forest (Mittermeier, 1977).

Ateles also apparently prefer undisturbed high forest in other parts of South America (e.g. , Janson, 1975; Bernstein, et al., 1976; Hernández-Camacho and Cooper, 1976). They are usually not found in areas where human activity has resulted in forest destruction. Their absence from most areas where there has been human activity may be a result of the hunting pressure that frequently accompanies partial habitat destruction, rather than the monkeys' inability to survive in isolated patches of habitat. Ateles geoffroyi from Central America is apparently more flexible in choice of habitat than most of its South-American relatives. Freese (1976) observed it in evergreen, semi-deciduous and sometimes even deciduous forest in Costa Rica, and Eisenberg and Kuehn (1966) and Alvarez del Toro (1977) report it even from mangrove forest in Chiapas, Mexico. The available evidence indicates that all Ateles species prefer undisturbed high forest. However, where such habitat is limited in extent, where hunting pressure is light or non-existent, and where other primate competitors are few in number, it appears to be able to live in several other forest types as well.

In the Voltzberg region, Ateles is predominantly seen in high rain forest (fig. 18A). Of all first sightings, $92.6 \%$ occurred in high 

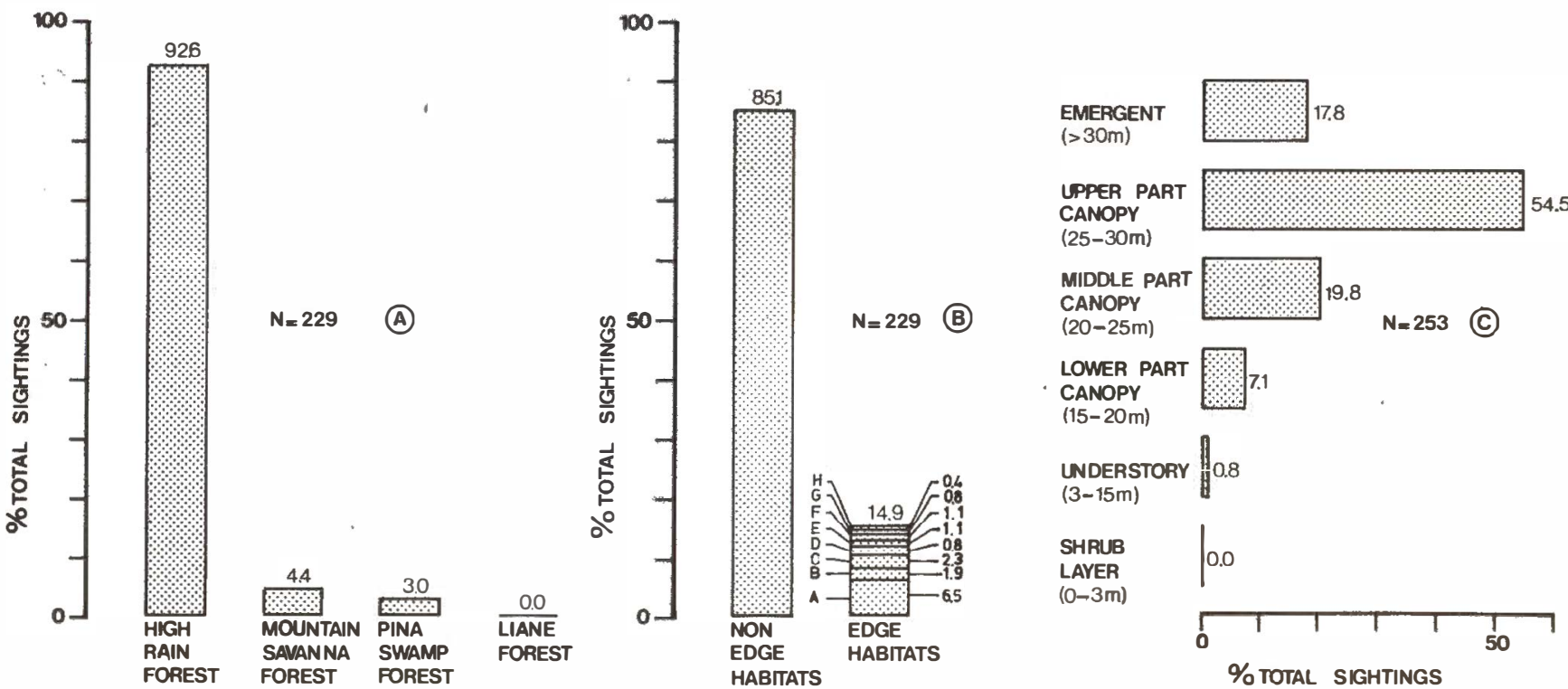

Figure 18 Habitat preferences of Ateles p. paniscus in the Voltzberg study area. Data are based on first sightings only.

A. Forest type preferences - B Utilization of edge habitats $(\AA=$ high forest, edge pina swamp forest $\cdot B=$ high torest, edge liane forest: $\mathrm{C}=$ high forest, edge clearing; $\mathrm{D}=$ high forest, edge mountain savanna forest; $E=$ pina swamp torest, edge high forest; $F=$ mountain savanna forest, edge clearing; $G$ = mountain savanna forest, edge liane forest; $H$ = mountain savanna forest, edge high forest) - C. Utilization of forest strata. 
forest. It entered high mountain savanna forest only occasionally (4.4\%), particularly when some food species typical of this formation was fruiting. During the long dry season, this vegetation type had almost nothing to offer for spider monkeys and consequently they were very rarely seen there at this time of year. The third forest type in which Ateles was occasionally seen was pina swamp forest $(3.0 \%)$. Ateles did not avoid this type of forest while traveling; instead it used the flexible stems of the pina palms (Euterpe oleracea) as jumping-poles while hanging in the small crowns. Several tree species typical of pina swamp forest (e.g., Carapa procera, Eperua falcata, Euterpe oleracea, Pterocarpus officinalis and Virola surinamensis) provide seasonal sources of food for Ateles, and consequently the animals visit this formation more often during certain times of the year. I never observed Ateles in the other habitats available in the Voltzberg region (e.g., liane forest, low forest and 'rocksavanna'). In the Lolopasi area, Ateles was seen in riverbank high forest many times, but never within $150 \mathrm{~m}$ of the riverbank itself. However, its home range was situated with its long axis perpendicular to the riverbank and its center beyond the boundaryline between riverbank high forest and high forest, at about 700 $m$ inland. Whether this was the original situation resulting from the habitat being relatively poor in nutritious fruits or whether it has been influenced by light hunting pressure was not clear.

Of all Surinam monkey species, Ateles paniscus is the most restricted in habitat (Mittermeier and Van Roosmalen, 1981). Only the bearded saki (Chiropotes satanas) shows a somewhat similar restriction in habitat choice. All other Surinam monkey species visit liane forest and/or low forest with some frequency.

\subsection{Edge Preferences}

At the Voltzberg study site, an area rich in edge habitats, Ateles did not show any preference for these situations (fig. 18B). In $85.1 \%$ of all first sightings, it was 'seen in non-edge habitats and only in $14.9 \%$ was it seen in edge habitats. Of these, only $6.1 \%$ were in edges with clearings or liane forest. Where hunting had not taken place in the recent past, spider monkeys did not appear to be afraid of exposing themselves at edges of open areas. Several of the sleeping trees, which were greatly prefered at a particular time of the year, were situated at the very edge of clearings and/or 
liane forest. The only reason for their apparent avoidance of edge habitats may be that these habitats do not offer them appropriate food resources. Fruits found at edges are mostly of the low-nutritious berry type or dry wind-dispersed, which are not of particular interest to a predominantly frugivorous species such as Ateles (Van Roosmalen, in prep.).

Of the eight Surinam monkey species, only Saguinus midas was seen more often in edge habitats than in non-edge habitats (Mittermeier and Van Roosmalen, 1981). Saimiri sciureus and Cebus apeela are also apparently partial to edge habitats. They tend to follow the edges of liane forest complexes in search of insects. Alouatta seniculus and Pithecia pithecia occurred somewhat more often in edge habitats than Ateles did and Chiropotes satanas and Cebus nigrivittatus less often.

\subsection{Vertical Stratification}

Ateles is primarily an animal of the upper levels of the forest with $17.8 \%$ of total first sightings in emergents and $54.5 \%$ in the upper part of the canopy. The middle levels of the forest were less frequently used, with $19.8 \%$ of total first sightings in the middle part of the canopy and only $7.1 \%$ in the lower part of the canopy. Ateles very rarely entered the understory and never descended below $12 \mathrm{~m}$, and was seen in the understory in only $0.8 \%$ of total first sightings (fig. 18C).

Of the other Surinam monkey species, only Chiropotes satanas shows a vertical distribution very similar to that of Ateles, with $60.2 \%$ of total first sightings $(n=93)$ in the two uppermost strata. All other Surinam monkey species prefer the middle and/or lower strata (Mittermeier and Van Roosmalen, 1981).

In summary, Ateles is the most restricted species in the Voltzberg area. It occurs almost exclusively in high forest, rarely enters edges and occurs primarily in the upper levels of the canopy and in emergents. Given the abundance of edges in the Voltzberg area (fig. 17), it actually seems to be avoiding these situations, which is probably due to low supply of preferred fruit. 


\section{DIET AND FEEDING BEHAVIOR}

\subsection{Food Categories}

In this study, spider monkey food was divided into eight categories: (1) truits -- including young seeds, mature seeds, stones, plumula, aril, exocarp, mesocarp, endocarp, pericarp, perigonium, infructescence, compound fruit, fig. , pseudotruit, juice and columella, (2) flowers - including floral buds, mature flowers and inflorescences, (3) leaves - including leat buds, young or flush leaves, mature leaves, petioles and tender shoots, (4) pseudobulbs, (5) aerial roots, (6) bark and decaying wood, including fresh bark, decaying and decayed bark and wood, and rotten leat bases, (7) honey, and (8) insects.

In table I, all food species and the part(s) eaten by spider monkeys are listed, ordered alphabetically first to family, then to genus and last to species. In total, 207 food plant species were identified of which $68.1 \%$ are trees, $25.6 \%$ lianes and stranglers, $1.0 \%$ herbaceous twiners and $5.3 \%$ epiphytes.

Of the 58 plant families providing the spider monkeys with food, Moraceae was the best represented with 29 species, followed by Mimosaceae (18 species), Bignoniaceae (12 species), Guttiferae ( 9 species) and Caesalpiniaceae (8 species). Ranked according to percentage of total feeding records, most important families were Moraceae ( $11.8 \%$ ), Mimosaceae (11.6\%), Myristicaceae (10.9\%), Sapotaceae (8.6\%), Meliaceae (5.8\%) and Burseraceae $(5.6 \%)$ (table II).

Among the genera, Virola comes first, followed by Inga, Ecclinusa, Guarea, Tetragastris, Cecropia, Dimorphandra, Philodendron, Bagassa, Achrouteria and Laetia (table III).

Of all food species recorded, Virola melinonii (Myristicaceae) is most important with $10.7 \%$ of total number of feeding records, followed by Guarea grandifolia (Meliaceae) with 4.0\%, Ecclinusa guianensis (Sapotaceae) with 3.3\%, Cecropia sciadophylla (Moraceae) with 3.2\%, Dimorphandra pullei (Caesalpiniaceae) with $3.1 \%$, Bagassa guianensis (Moraceae) with $2.8 \%$, Inga edulis (Mimosaceae) and Achrouteria pomifera (Sapotaceae) with $2.6 \%$, In- 
ga alba (Mimosaceae) and Tetragastris panamensis (Burseraceae) with $2.5 \%$. About 85 species were recorded only once or twice during the study period, and therefore account for less than $0.1 \%$ of total number of feeding records (table IV).

As shown in figure 19, Ateles p. paniscus is predominantly frugivorous in its overall diet, feeding on fruits in $82.9 \%$ of total feeding records $(\mathrm{N}=2,287)$. Flowers and leaves play a minor role, accounting for $6.4 \%$ and $7.9 \%$ respectively, but seasonally they can be important. Other foods are bark (1.7\%), decaying wood $(0.3 \%)$, pseudobulbs $(0.1 \%)$, aerial roots $(0.2 \%)$, honey $(0.2 \%)$, termites $(0.1 \%)$ and caterpillars $(0.1 \%)$.

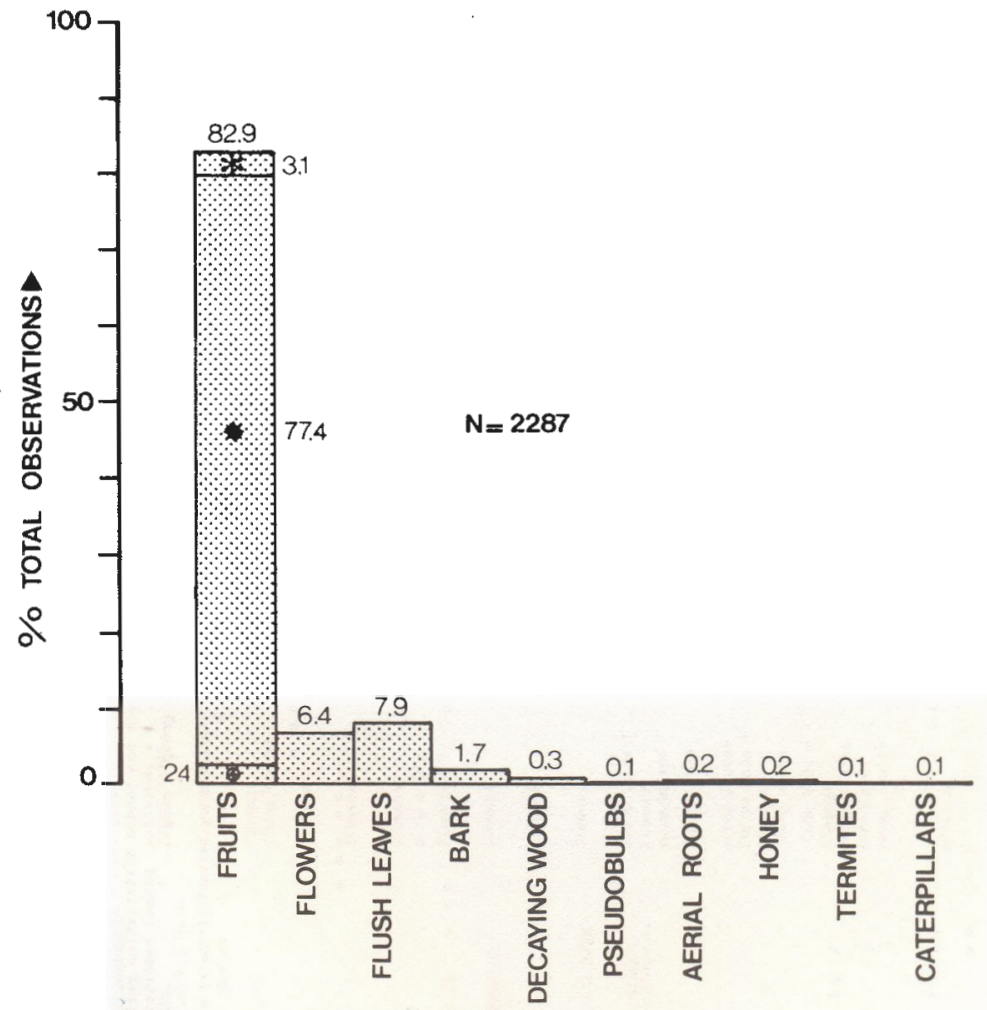

Figure 19 Annual utilization of different foods in Ateles p. paniscus, expressed as the percentage of the total number of feeding records for each food category ( $*=$ seeds predated, $*=$ seeds dispersed endochorically, $=$ seeds dropped under the parent plant). 
Table I. Food liat of Atelea $\mathrm{E}$. paniseun in Surinam

- not Invantoried fn Voltzberg study area, $x=$ seed dispersion/predation, - = nil, - = maximum height in meters, () = not occurring in Voltzberg study area, $\mathbf{m}=$ number of food plants used by the study group, sp. = species, anfruct, = infructescences, inflor. = inflorescences, h.riverb.f. = riverbank high forest, h. wount. anr.f, = high

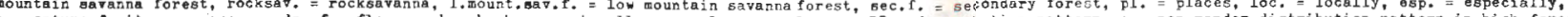

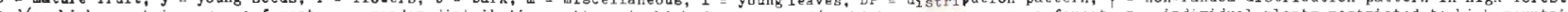
and or high moutain savang torest, - random distribution pattern in high forest and or high mountain savanta forest, a $=$ individual plants restricted to high wountai and/or high mountain savanta forest, $\mathbf{n}=$ individual
randomly distributed throughout the group's range.

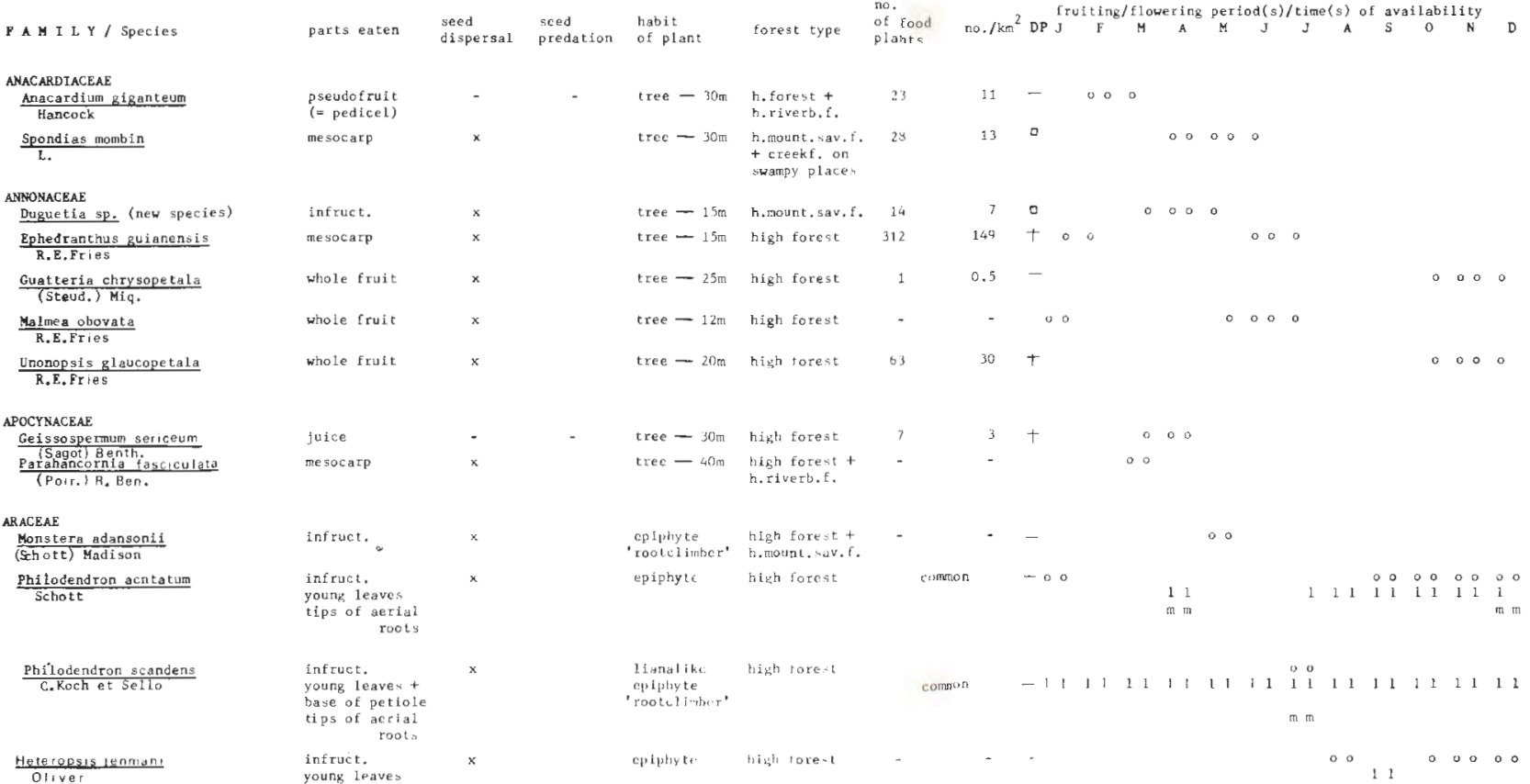




\begin{tabular}{|c|c|c|c|c|c|c|c|c|c|c|c|c|c|c|c|c|c|c|c|}
\hline FAMIL Y / Species & parts eaten & seed & seed & habit & & $\begin{array}{l}\text { no. } \\
\text { of food }\end{array}$ & & ${ }^{2} D P$ & & & $\int_{4}$ & ing & perio & $\stackrel{\operatorname{cod}(s) /}{\mathrm{J}}$ & time (s) & ) of av & $\begin{array}{l}\text { ava i labi } \\
5 \quad 0 \quad\end{array}$ & ility & 0 \\
\hline FAMILYT Species & parts eaten & oispersial & predacion & of p1ant & forest type & plants & $\pi 0.7 \mathrm{~km}$ & & & & $M$ & $\mathrm{~A}$ & M & $\mathrm{J}$ & & & 50 & $N$ & 0 \\
\hline \multicolumn{20}{|l|}{ BURSERACEAE } \\
\hline$\frac{\text { Protium aeglectum }}{\text { Swart }}$ & mesocatp & $\mathrm{x}$ & & tree $-20 \mathrm{~m}$ & high forest. & 311 & 145 & t & & & & 00 & 0 & & & & & & \\
\hline$\frac{\text { Protium polybotryum }}{\text { (Turcz.) Engl. }}$ & mesocarp & $\mathrm{x}$ & & tree $-25 m$ & high forest & 217 & 103 & + & & & & 0 & 00 & & & & & & \\
\hline$\frac{\text { Tetragastris altissima }}{\text { (Aubl.) Swart }}$ & mesocaxp & $\mathrm{x}$ & & tree $-30 \mathrm{~m}$ & high forest & 1631 & 777 & $T$ & & & 0 & 00 & $\circ$ & 00 & & & & & \\
\hline$\frac{\text { Tetragastris panamensis }}{\text { (Engl.)o.Kuntze }}$ & mesocarp & $x$ & & tree $-25 \pi$ & high forest & 280 & 133 & 十 & & & & 10 & 00 & 00 & & & & & \\
\hline \multicolumn{20}{|l|}{ CACTACEAE } \\
\hline Hylocereus sp. a. & $\begin{array}{l}\text { flowers } \\
\text { whole fruit }\end{array}$ & $\mathbf{x}$ & & epiphyte & $\begin{array}{l}\text { high forest }+ \\
\text { h.mount.sav.f. } \\
\text { esp.at edges }\end{array}$ & + & - & $\mathrm{f}$ & $f f \quad f$ & $f \pm$ & $f_{0}^{f f}$ & $\mathrm{f} i$ & if & & & f $\mathrm{i}$ & I $\quad f \quad f$ & E $\mathrm{f}$ & $\mathrm{rf}$ \\
\hline Hylocereus sp. b. & $\begin{array}{l}\text { whole fruit } \\
\text { flowers }\end{array}$ & $x$ & & epiphyte & $\begin{array}{l}\text { high forest }+ \\
\text { h.mount.sav.f. } \\
\text { esp.at edges }\end{array}$ & - & - & & & .0 & $\circ \circ$ & & & & & $\mathrm{fE}$ & E I $\mathrm{E}$ & & \\
\hline$\frac{\text { Pereskia aculeata }}{\text { Mill }}$ & $\begin{array}{l}\text { Elowers } \\
\text { whole fruit }\end{array}$ & $x$ & & Liana & $\begin{array}{l}\text { h. mount. sav. } \mathrm{f} \text {. } \\
\text { esp.at edges }\end{array}$ & - & - & & 0 & $\circ 0$ & $\dot{0}$ & & & & & & & $f$ & $\mathrm{f}$ \\
\hline \multicolumn{20}{|l|}{ CAPPARACEAE } \\
\hline$\frac{\text { Capparis maroniensis }}{\text { Bert. }}$ & mesocarp & $x$ & & tree $-30 \mathrm{~m}$ & high forest & 304 & 145 & T 0 & 000 & 00 & 00 & 00 & o & & & & & & \\
\hline \multicolumn{20}{|l|}{ CARICACEAE } \\
\hline$\frac{\text { Jacaratia spinosa }}{\text { (Aub1.) A.DC. }}$ & $\begin{array}{l}\text { whole fruit } \\
\text { young leaves }\end{array}$ & $x$ & & tree $-20 \mathrm{~m}$ & $\begin{array}{l}\text { hish forest }+ \\
\text { h, mount.sav.t. } \\
\text { esp.at edges }\end{array}$ & 7 & 3 & 0 & 11 & & & & 90 & & & & & & \\
\hline CELASTRACEAE & & & & & & & & & & & & & - & & & & & & \\
\hline$\frac{\text { Cheiloclinium cognatum }}{\text { (Miers) A.C. Smith }}$ & (young) seeds & & $\mathrm{x}$ & tree $-12 \mathrm{~m}$ & hish forest & 20 & 10 & व & & & & $y$ & $y y$ & & & & & & \\
\hline$\frac{\text { Cheiloclinium hippocrateoldes }}{\text { IPeyr.t A.C. Smith }}$ & me soc arp & $x$ & & liana & h.riverb.f. & 0 & 0 & & & & 60 & $00)$ & & & & & & & \\
\hline$\frac{\text { Cheiloclinium podostemmumi }}{\text { (Sandw.) A.C. Smith }}$ & mesocatp & $\mathrm{x}$ & & liana & $\begin{array}{l}\text { high forest + } \\
\text { h.riverb.f. }\end{array}$ & - & - & & & & & & 00 & & & & & & \\
\hline Cheiloclinium a nomalum & mesocatp & $\mathrm{x}$ & & liana & high forest & $1^{*}$ & $0.5^{*}$ & - & & & & 。 & o & & & & & & \\
\hline $\begin{array}{l}\text { Miers } \\
\text { Amphizoma sp. }\end{array}$ & me socarp & $x$ & & liana & high forest & $2^{*}$ & $1^{ \pm}$ & - & & & 00 & & & 00 & & & & & \\
\hline Maytenus SP. & aril & $\mathbf{x}$ & & tree $-30 \mathrm{~m}$ & $\begin{array}{l}\text { high forest }+ \\
\text { h.mount, sav. f. }\end{array}$ & 3 & 1 & $\square$ & & & o 0 & & 00 & & & & & & \\
\hline \multicolumn{20}{|l|}{ CHRY SOB ALANACEAE } \\
\hline$\frac{\text { Couepia caryophylloldes }}{\mathrm{R} \text {, Ben. }}$ & $\begin{array}{l}\text { exocarp } \\
\text { me socarp }\end{array}$ & - & - & tree $-25 \mathrm{~m}$ & high forest & 99 & 47 & T & & & & & & & & & & 00 & o \\
\hline$\frac{\text { Licania densiflora }}{\text { Kleinh. }}$ & $\begin{array}{l}\text { exocatp }+ \\
\text { mesocarp }\end{array}$ & - & - & tree $-30 \mathrm{~m}$ & high forest & - & - & & & & & 00 & & & & & & & \\
\hline$\frac{\text { Eicanita majuscula }}{\text { Sagot }}$ & $\begin{array}{l}\text { exocarp }+ \\
\text { mesocarp } \\
\text { bark }\end{array}$ & - & - & tree $-30 \mathrm{~m}$ & $\begin{array}{l}\text { high forest }+ \\
\text { h.mount, sav.f. }\end{array}$ & 67 & 32 & 十。 & 000 & $\circ 0$ & $\circ 0$ & 0 & & & & & & $\begin{array}{l}0 \\
0 \\
0\end{array}$ & 00 \\
\hline$\frac{\text { Licania micrantha }}{\text { Mig. }}$ & $\begin{array}{l}\text { young leaves } \\
\text { bark }\end{array}$ & - & - & tree $-30 \mathrm{~m}$ & high forest & 49 & 23 & $T^{t}$ & b b $\quad$ b & $b \mathrm{~b}$ & & & & & b & & & $\begin{array}{ll}1 & 1 \\
b & b\end{array}$ & $b$ b \\
\hline$\frac{\text { Parineri campestris }}{\text { Aub1. }}$ & $\begin{array}{l}\text { exocarp }+ \\
\text { mesocarp }\end{array}$ & - & - & tree $-25 m$ & $\begin{array}{l}\text { high forest }+ \\
\text { h.riverb.f. }\end{array}$ & 12 & 6 & + & & & $\circ 0$ & 00 & & & & & & & \\
\hline$\frac{\text { Parinari excelss }}{\text { Sabine }}$ & $\begin{array}{l}\text { exocarp }+ \\
\text { mesacarp }\end{array}$ & - & - & tree -400 & high forest & 22 & 10 & 4 & & & $\circ 0$ & & & & & & 0 & & \\
\hline
\end{tabular}




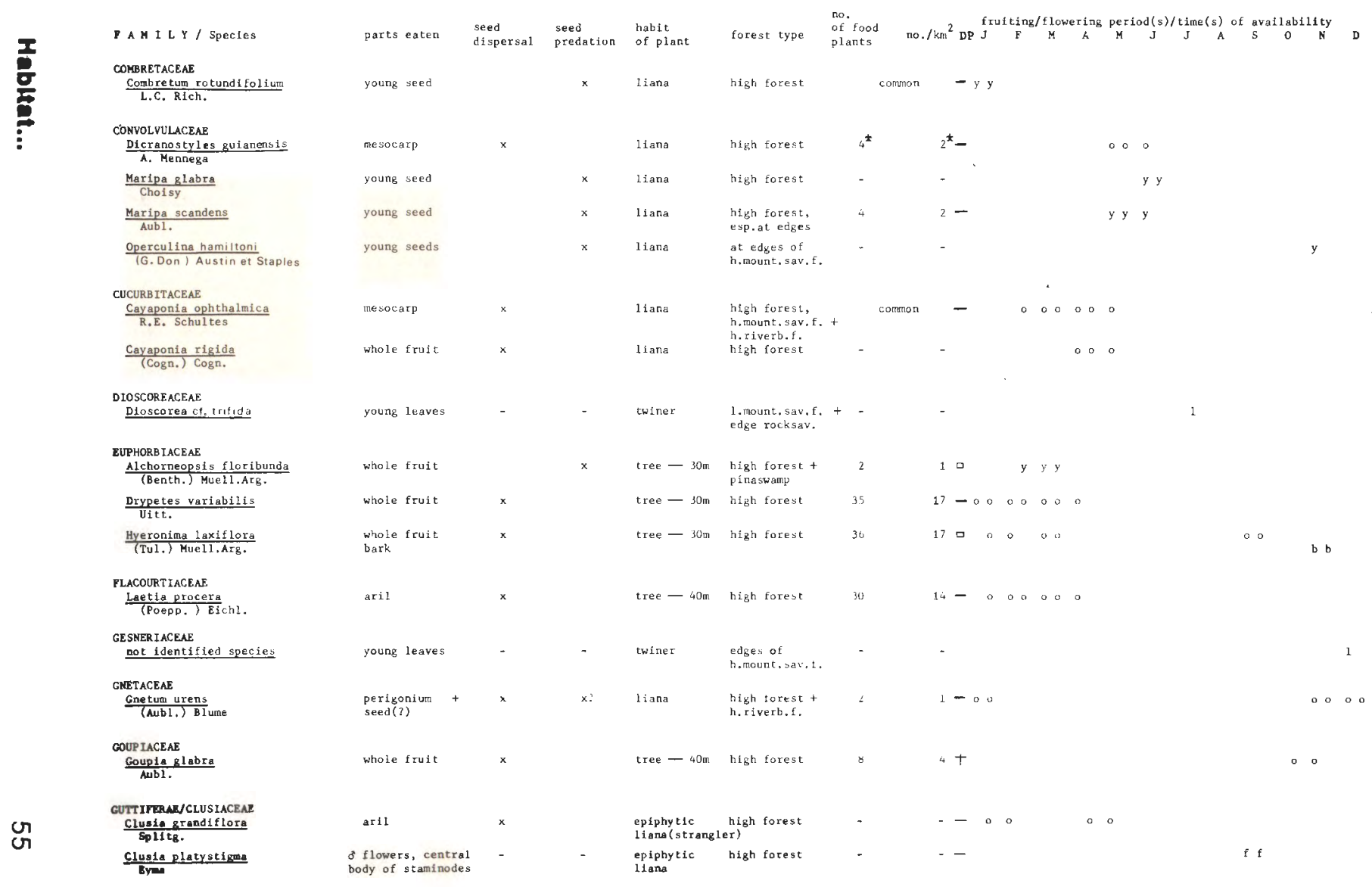


FA M I Y / Species

$\frac{\text { Clusia Iepranth. }}{\text { Mart. }}$

$\frac{\text { Clusia scrobiculata }}{\text { h. Ben. }}$

Clusia 5.

GUTTIFERAE

$\frac{\text { Platoria insignis }}{\text { Mart. }}$

$\frac{\text { Rheedia benthamiana }}{\text { Planch.et Triana }}$

$\frac{\text { Rheedia macrophyl la }}{\text { (Mart.) Planch,et Triana }}$

$\frac{\text { Sympnonia globulifera }}{\text { L.f. }}$

HUMTR TACEAE

Sacoglottis cydonioides

vaneanea parviflora

ICACINACEAE

$\frac{\text { Leretia cordata }}{\text { Veli. }}$

LAURACEAE

$\frac{\text { Licaria canella }}{\text { (Meissn.) Kosterm. }}$

Nectandra kunthian

(Nees) Kosterm

$\frac{\text { Ocotea wachenheini } 1}{\text { R. Ben. }}$

ECYTHIDACEAE

$\frac{\text { Couratari guianensis }}{\text { Aubl. }}$

Couratari stellata

Eschweilera congestifiora

$\frac{\text { Eschweilera corrugata }}{\text { (Port.) Miers }}$

$\frac{\text { Eschweilera poiteaui }}{\text { (Berg) Ndz. }}$

Gustavia hexapetala

(Aubl.) J.E. Sarith

$\frac{\text { Lesychts davisil }}{\text { Sandw. }}$

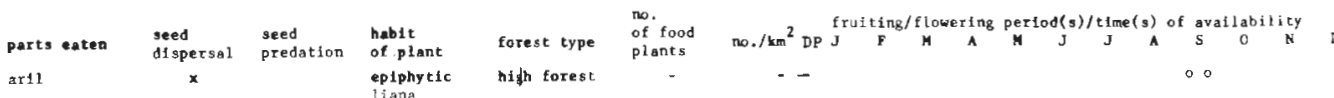

epiphytic high forest

corrnon

$-000000$

epiphytic high forest

$-$

E 1

tree $-35 \mathrm{~m}$ h.riverb.f.

$1-$

0000

tree $-15 \mathrm{~m}$ hieh fortet

$3-$

tree -20 m high torest

tree $-25 \mathrm{~m}$ high forest +

$41-$

10

flovers

whole frui

bark

tree - jom high forest

tree--40m highforest

whole frui

liana

bigh fore:t

100000000

young leaves

tree $-25 m \quad$ h.mount.sav..

10

tree $-30 \mathrm{~m}$ homount, sav. f.

110

tree $-28 \mathrm{~m}$ high forest

$1-$

$\circ 0$

young seeds

young seeds

bark

young seeds +

young seeds +

ari1

young seeds +

ari1

mesocarp +

funicle

$\begin{aligned} \text { tree }-50 m & \begin{array}{l}\text { high forest }+ \\ \text { h.riverb. }\end{array}\end{aligned}$

h. riverb. $f$.

tree $-45 m$ high forest

403

tree $-25 \mathrm{~m}$ high forest

higb forest $+\quad 761$

$\begin{array}{ll}\text { tree }-25 \mathrm{~m} & \text { high forest } \\ & \text { pinaswamp } \\ \text { tree }-35 \mathrm{~m} & \text { high forest }\end{array}$

336

tree $-15 \mathrm{~m}$ high forest +

h.mount. sav. E.

$\begin{array}{ll}\text { tree }-30 \mathrm{~m} & \begin{array}{l}\text { high forest }+ \\ \text { h.ziverb.f. }\end{array}\end{array}$

-


F A M I L Y / Species

parts eaten

LEGUMI NOSAE/CAE SALP INIACEAE $\frac{\text { Copsifera epunctata }}{\text { Ansh }}$

aril

Copaifera guianensis

Cynometrá marginata

$\frac{\text { Dialium guianense }}{\text { (Aubl.) Steud. }}$

Dimorphandra pule

$\frac{\text { Eperus falcata }}{\text { Aubl. }}$

Eperua rubiginosa

Miq.

$\frac{\text { Peltagyne venasa }}{\text { (Vah1) benth. }}$

LEGLMINOSAE/MIMOSACEAE

Cedrelinga caten!formıs

Enterolobium schomburgki

$\frac{\text { Inga acrocephala }}{\text { steut. }}$

$\frac{\text { Inga cinnamomea }}{\text { Spruco ex Benth. }}$

$\frac{\text { Inga alba }}{\text { (Sw.) willd }}$

Inga bourgoni

$\frac{\text { Inga } c F \text {, capitata }}{\text { Desv. }}$

$\frac{\text { Inga coriacea }}{\text { (Pers.) Desv. }}$

Inge edulis

Inga leivealscins

Benth.

$\frac{\text { Insá pezizifera }}{\text { Benth }}$

U

$\frac{\text { Inga rubizinosa }}{\text { (L.C.Rich.) DC. }}$

$\frac{\text { Inga stipularis }}{D C}$

$\frac{\text { Inga thibaudians }}{\mathrm{DC} .}$ young leaves

aril

mesocarp

plumula

floung leaves

plumula

aril

young seeds

inflor.

mesocarp

ne socarp

me soc arp

mesocarp

mesocarp

the ios a rp

me vocurp

II sulatp
bath

nie socarp

me soc arp

me : oc arp

mesocarp tree $-40 \mathrm{~m}$ high forest

tree $-40 \mathrm{~m}$ high forest + h. riverb. $f$.

tree - 20m high forest, esp-on swampy

tree - 30 places

tree -45 m high forest

tree - 30m high forest + pi na swamp tree $-30 \mathrm{~m} \begin{aligned} & \text { h.riverb.f. } \\ & \text { creekforest }\end{aligned}+$

tree $-35 \mathrm{~m}$ high forest,
espolong creeks

tree -60 he high forest

tree - 40: high forest

tree -25 m high forest

tree - $20 \mathrm{~m}$ high forest,
espiat edges

tree - 35 m high forest

tree $-20 \mathrm{ml}$ high forest csp.dt edges

tree $-20 \mathrm{~m}$ high forest

tree $-25 \mathrm{~m}$ high forest

tree - 30m high forest, esprat edgen her. $f$.

tree - $25 \mathrm{~m}$ hish Forest, esp.alon

tree $-25 \mathrm{ml}$ high torest

tree $-25 \mathrm{~m}$ high forest

tree - 25m high forest t

$\begin{array}{ll}\text { tree }-25 \mathrm{~m} & \begin{array}{l}\text { high forest } \\ \text { espogt edges }\end{array}\end{array}$

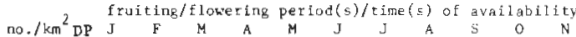

11

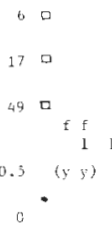

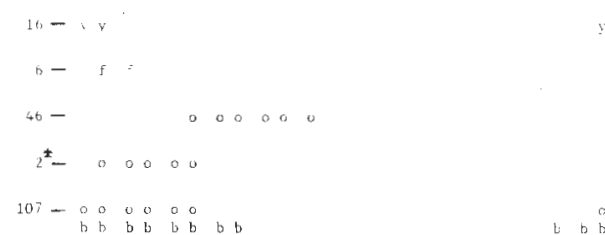

o 0 
FAMILY / Species

Piptadenia suaveolens

Parkig nitida

Parkis pendula

(wirld.) Benth.

$\frac{\text { Plthecellobium jupunba }}{\text { (Willd.) Urb. }}$

LEGUMINOSAE/F AP IL IONACEAE

$\frac{\text { Dioclea macrocarpa }}{\text { Huber }}$

$\frac{\text { Dipteryx odorata }}{\text { (Aubl.) Willd. }}$

$\frac{\text { Hymeno lobium petraeum }}{\text { Ducke }}$

$\frac{\text { ormosia paraensis }}{\text { Ducke }}$

$\frac{\text { Flatymiscium ulei }}{\text { Hamms }}$

$\frac{\text { Pterocarpus officinalis }}{\text { Jacq. P.P.rs.santalinoides) }}$

Yetaireopsis speciosa

$\frac{\text { Votaireopsis speciosa }}{\text { Ducke }}$

\section{LOGNIIACEAE}

$\frac{\text { Strychnos tomentosa }}{\text { Benth. }}$

LoRAMTHACEAE

not identified species

MARCGRAVIACEAE

$\frac{\text { Norantea guianensis }}{\text { Aúbl. }}$

ME LASTOMATACEAE

Bellucia grossularioides

MELIACEAE

$\frac{\text { Carapa procera }}{\text { DC. }}$

Cedrelá odoreta

Guakes grandifolia

Cuareakunthiana
A. Juss. parts eaten

seed

$\begin{array}{ll}\text { seed } & \text { habit } \\ \text { predation } & \text { of plant }\end{array}$

forest type

no. plants

inflor.
(young buds)

inflor.

inflor.

young leaves

bark

young seeds

young leaves

young leaves

young seed

flowers

young leaves

young leaves

me socarp

young fleshy

flowers

whole fruit

f lowers

young seeds

young seeds +
columella

flovers

aril

$$
\begin{array}{ll}
\text { tree }-50 \mathrm{~m} & \text { high forest } \\
\text { tree }-50 \mathrm{~m} & \text { high forest } \\
\text { tree }-45 \mathrm{~m} & \text { high forest } \\
\text { tree }-35 \mathrm{~m} & \text { high forest }
\end{array}
$$

high forest

tree $-30 \mathrm{~m}$ high forest

tree - $50 \mathrm{~m}$ high forest $\begin{aligned} \text { tree }-30 m & \begin{array}{l}\text { high forest } \\ \text { esp. along } \\ \text { creks }\end{array}\end{aligned}$

$\begin{array}{ll}\text { tree }-30 \text { mi } & \text { creeks } \\ \text { high forest }\end{array}$

tree - ins high forest

high forest 70

liana

high forest

high forest

eptithytel

Liana

high forest, h.riverb.f.

tree $-20 \mathrm{~m}$ h.mount.sav. f. on graníte

tree $-30 \mathrm{~m}$ high forest on 417 swampy places + swampy places
pinastamp

tree $-40 \mathrm{~m}$ high forest high forest 9

tree - 30m high forest 449

$$
21 \text { - F } f
$$$$
5-
$$$$
7-
$$$$
17-
$$

$\begin{aligned} \text { tres - 30n pinaskamp }+ & \\ \text { h, mount sav. } & \end{aligned}$ $\begin{array}{ll}\text { tree - } 30 \mathrm{~m} & \begin{array}{l}\text { high forest }+ \\ \text { h.tiverb.f. }\end{array}\end{array}$ (y)

F f

$10-$

520

$33-111$
$1^{*}-0$

$199+f_{f}$

$4-y y$

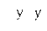

1112111

$214+$

$\begin{array}{llllllllllllllllll}f & f & & & & \\ 0 & 0 & 0 & 0 & 0 & 0\end{array}$

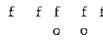




\begin{tabular}{|c|c|c|c|c|c|c|c|c|c|c|c|c|c|c|c|c|c|c|c|c|}
\hline 7 & F A M I L Y / Species & parts eaten & seed & seed & habit & & $\begin{array}{l}\text { no. } \\
\text { of food }\end{array}$ & & fru & $\begin{array}{c}\text { ruiting } \\
\mathrm{F}\end{array}$ & & verint & $\therefore$ peri & $\operatorname{ods}(s)$ & time & (s) of & & ilabi & Lity & \\
\hline$\frac{1}{2}$ & - & 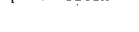 & dispersal & predation & of plant & iorent cype & Wants & no. $/ \mathrm{km}^{2} \mathrm{D}$ & DP J & $\mathrm{F}$ & M & A. & $M$ & J & & A & $s$ & 0 & $\mathrm{H}$ & D \\
\hline$\ddot{z}$ & $\frac{\text { Irlchllia martiana }}{\text { C.DC. }}$ & aril & $\mathrm{x}$ & & tres: -25 rin & $\begin{array}{l}\text { h., moent. Lav.t. } \\
\text { h. riverb,f. }\end{array}$ & $1^{*}$ & $0.5^{*}$ & & & & 00 & & & & & & & & \\
\hline 2 & $\frac{\text { Trlchll1a quadrijuga }}{\text { H.B.K. }}$ & aril & $x$ & & $\operatorname{trcs}-25 m$ & high torest & 102 & $44^{4}-7$ & & 00 & 00 & & & & & & & & & \\
\hline & $\frac{\text { Trichilia surinamensi.s }}{(M 1 q .) C . D C .}$ & whole Iruic & x & & tree - & 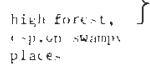 & 102 & & -60 & & & & & & & & & & & 00 \\
\hline & $\begin{array}{l}\text { KENISPERMACEAE } \\
\frac{\text { Abuta a grandifoli.a }}{\text { (Mart.) Sands, }}\end{array}$ & mesocarp & $x$ & & tiana & $\begin{array}{l}\text { hich foren, } \\
\text { rippat etigen }\end{array}$ & $4^{\star}$ & $2^{*}-$ & - & & 00 & & & & & & & o & o & \\
\hline & MORACEAE & & & & & & & & & & & & & & & & & & & \\
\hline & $\frac{\text { Bagassa guianensis }}{\text { Aub1. }}$ & $\begin{array}{l}\text { unripe + ripe } \\
\text { infruct. }\end{array}$ & $\mathrm{x}$ & & tree $-45 \pi$ & high torest & 30 & $17-$ & $-\approx 0$ & o & & & & o o & o & i & $\begin{array}{ll}0 & 0 \\
y & y\end{array}$ & $\begin{array}{ll}0 & 0 \\
y & y\end{array}$ & y & $\circ \circ$ \\
\hline & $\frac{\text { Brosimum, lactescens }}{\text { (S. Moore) C.C. Berg }}$ & inf ruct. & $\mathrm{x}$ & & tree $-35 \mathrm{~m}$ & $\begin{array}{l}\text { high torest } t \\
\text { h.mount,sdv.f. }\end{array}$ & 32 & 150 & 0.0. & & & & & & & & & & $\circ 0$ & 00 \\
\hline & $\frac{\text { Brosimum parinarioides }}{\text { Ducke }}$ & inf ruct. & $\mathrm{x}$ & & tree $-40 \mathrm{~m}$ & high forest & 12 & $6-$ & - & & & & & & & & & & $\circ 0$ & 。 \\
\hline & $\frac{\text { Cecropla scladophy Lla }}{\text { Mart. }}$ & $\begin{array}{l}\text { infruct. } \\
\text { young leaves }\end{array}$ & $x$ & & tree $-30 \mathrm{~m}$ & $\begin{array}{l}\text { high forest } \\
\text { (old treefall } \\
\text { cleorings) }\end{array}$ & 40 & $19-$ & $-\approx 0$ & $\begin{array}{ll}0 & 0 \\
1 & 1\end{array}$ & 00 & 00 & & & & & $\circ 0$ & $\circ 0$ & $\circ 0$ & 00 \\
\hline & $\frac{\text { Cecropia latrloba }}{\text { Miq. }}$ & infeuct. & $\mathrm{x}$ & & tree $-25 \mathrm{~m}$ & $\begin{array}{l}\text { sec.f., at ed- } \\
\text { ges of high } \\
\text { forest + } \\
\text { h.mount.sav.f. }\end{array}$ & 39 & 190 & 口.0 & 0 & & & & 00 & & & & & 00 & $\circ \circ$ \\
\hline & $\frac{\text { Clarisia racemosa }}{\text { R.et P. }}$ & whole fruit & $x$ & & tree $-40 m$ & high forest & 19 & & - & & & & 00 & & & & & & $\circ 0$ & 0 \\
\hline & $\frac{\text { Coussgpoa angustifolia }}{\text { Aub1. }}$ & inf ruct. & $\mathrm{x}$ & & $\begin{array}{l}\text { epiphytic } \\
\text { treefliana }\end{array}$ & high Eorest & 1.5 & $7-$ & - & & & & & & & o & 00 & o 0 & o & \\
\hline & $\frac{\text { Eoussapos asperifolia }}{\text { Tréc. }}$ & $\begin{array}{l}\text { infruct. } \\
p^{2} \text { stislate in- } \\
\text { florescences }\end{array}$ & $\mathrm{x}$ & & $\begin{array}{l}\text { epiphytic } \\
\text { treefliana }\end{array}$ & $h_{i} b_{b}$ Eorest & 5 & $2-$ & - & & & & & & $f \mathfrak{f}$ & & & 口 & 00 & \\
\hline & $\frac{\text { Coussapoa Latifolia }}{\text { Aubl. }}$ & inf tuct. & $\mathrm{x}$ & & $\begin{array}{l}\text { epiphytic } \\
\text { tree /liana }\end{array}$ & high torest & a7 & $41-$ & & & & & & & & & & & $\circ \circ$ & 00 \\
\hline & $\frac{\text { Ficus guianensis }}{\text { Desv. exHam. }}$ & figs & $\mathrm{x}$ & & $\begin{array}{l}\text { epiphytic } \\
\text { tree/liana }\end{array}$ & $\begin{array}{l}\text { high forest, } \\
\text { on swampy } \\
\text { places }\end{array}$ & $1^{*}$ & $0.5^{ \pm}$ & & & & & & & & & & & & 。 \\
\hline & $\frac{\text { Ficus broadwayi }}{\text { Urb. }}$ & figs & $\mathrm{x}$ & & $\begin{array}{l}\text { epiphytic } \\
\text { tree/liana }\end{array}$ & high forest & $1^{\star}$ & $0.5^{\star}$ & & & & & & & & & & & $\circ$ & \\
\hline & $\frac{\text { Ficus citrifolia }}{\text { Mill }}$ & fig: & $\mathrm{x}$ & & $\begin{array}{l}\text { treelet/ } \\
\text { liana }\end{array}$ & $\begin{array}{l}\text { 1. niount. sav, t. } \\
\text { an granitee }\end{array}$ & $1^{ \pm}$ & $0.5^{*}$ & & & & & & & & & & & & 0 \\
\hline & $\frac{\text { Ficus gardnerians }}{\text { (M1q.) Miq. }}$ & figs & $\mathrm{x}$ & & liana & $\begin{array}{l}\text { high forest } \\
\text { (old treefall } \\
\text { clearings) }\end{array}$ & $1^{*}$ & $0.5^{\star}$ & & & & & & & & & & & 。 & \\
\hline & $\frac{\text { Ficus gomel Leira }}{\text { Kunth et Bouché }}$ & figs & $\mathrm{x}$ & & strangler & high forest & $2^{*}$ & $1^{*}$ & & & & & 0 & o & & & & & & \\
\hline$\pi$ & $\frac{\text { Picus 2nsipida }}{\text { W111d. }}$ & figs & $\mathrm{x}$ & & tree -30 m & $\begin{array}{l}\text { high forest }+ \\
\text { pinaswamp }\end{array}$ & $1^{ \pm}$ & $0.5^{\star}$ & & & & & & & & & & $\circ$ & & \\
\hline & $\frac{\text { Eicus malacocarpa }}{\text { stand1. }}$ & figs & $x$ & & $\begin{array}{l}\text { epiph.tree/ } \\
\text { strangler }\end{array}$ & high forest & $1^{2}$ & $0.5^{*}$ & & & & & & & & & & & 。 & \\
\hline
\end{tabular}




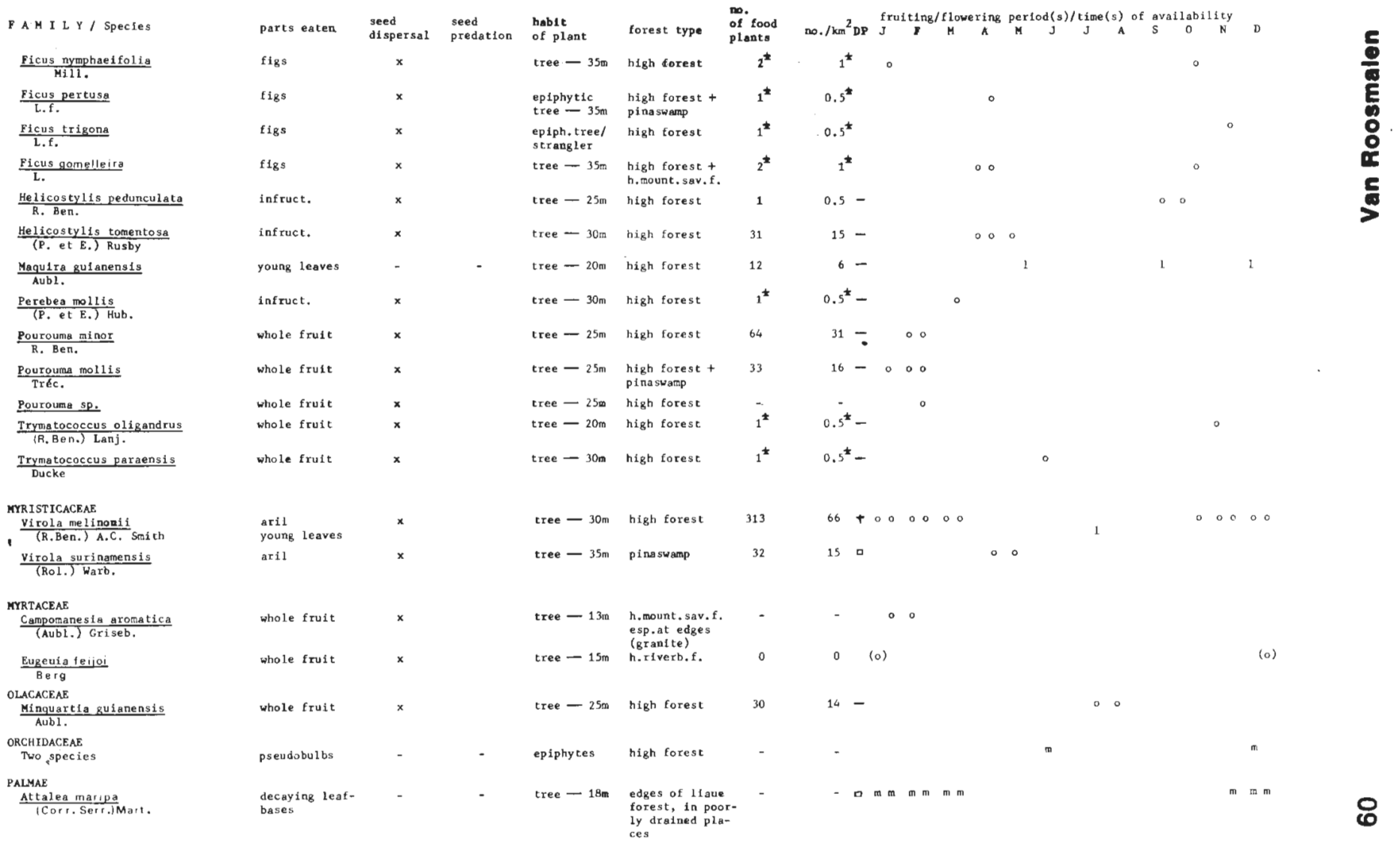




\begin{tabular}{|c|c|c|c|c|c|c|c|c|c|c|c|c|c|c|c|c|c|c|c|}
\hline 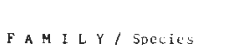 & purtsegten & seed & seed & Jabit & no. & if Eood & ${ }_{100} . / k_{\pi}^{2}{ }^{2}$ & $20 P^{1}$ & & ing $/$ fion & we $r i n g$ & perio & $\frac{\mathrm{J}}{\mathrm{ot}(\mathrm{s}) /}$ & 1 timet & (3) of & & Habil & lity & $D$ \\
\hline Euterpe oleracea & whole fruit & $\begin{array}{l}\text { dispersal } \\
x\end{array}$ & predation & of plant & porese cype pla & lant:. & $100.7 \mathrm{kmin}$ & & & & & & & & & & & $\mathrm{N}$ & $D$ \\
\hline Mart. & Whole fruit & & & tree -20 rin & $\begin{array}{l}\text { pinatharin, along } \\
\text { creek- }\end{array}$ & 5. lociatio & undant & 口 & & & & 0 & 00 & $c$ & & & & & \\
\hline$\frac{\text { Euterpe precatorie }}{\text { Mart. }}$ & whole truit & $x$ & & tree $-27 \mathrm{~m}$ & $\left\|i_{n}\right\|^{\prime}$ fute:-1 & - & - & & & & & & & & & o & 00 & o & \\
\hline$\frac{\text { Denocarpus bacaba }}{\text { Mart. }}$ & whole fruit & $\mathrm{x}$ & & treet $-22 n$ & $\begin{array}{l}\text { Wigh forent } t \\
\text { at edjoh of } i t\end{array}$ & 67 & 32 & - & & & & $\circ 0$ & 00 & & & & & & \\
\hline$\frac{\text { Iriartea exorthira }}{\text { Mart }}$ & whole fruit & $x$ & & tres $-30 \mathrm{~m}$ & pinaswamp & $2^{*}$ & $1^{*}$ & 0 & & $\circ$ & & $\circ$ & & & & & & & \\
\hline $\begin{array}{l}\text { Mart } \\
\text { PaSSTFLRACEAE }\end{array}$ & & & & & & & & & & & & & & & & & & & \\
\hline$\frac{\text { Passiflora glandulosa }}{\text { Cav. }}$ & whole fruit & $x$ & & $1 \mathrm{i}$ ana & $\begin{array}{l}\text { hijkb forest, } \\
\text { espot edges }\end{array}$ & - & - & & & & & & & 。 & & & & & \\
\hline \multicolumn{20}{|l|}{ PTPERACEAE } \\
\hline$\frac{\text { Peperomia glabe11a }}{\text { (St.) A. Dietr. }}$ & young leaves & - & - & epiphyte & $\begin{array}{l}\text { high forect, esp. } \\
\text { or toukhs of } \\
\text { cemergent trees }\end{array}$ & 1. alundatis & & 1 & & & & & & 1 & 111 & 11 & I 1 & 11 & 11. \\
\hline POLYGALACEAE & & & & & & & & & & & & & & & & & & & \\
\hline$\frac{\text { Moutabea guianensi: }}{\text { Aub1. }}$ & mésocarp & $x$ & & liana & thigh forest & 5 & 2 & -00 & & 0 & 0.0 & 0 & & 00 & & & & & \\
\hline \multirow{2}{*}{\multicolumn{20}{|c|}{$\begin{array}{l}\text { RUB IACEAE } \\
\frac{\text { Coussarea paniculata }}{\text { (Yahl) Standl. }}\end{array}$}} \\
\hline & whole fruit & $x$ & & tree $-12 \mathrm{~m}$ & tizh Earest & comnos & & $\dagger$ & & & $\circ$ & $\circ$ & & & & & & & \\
\hline$\frac{\text { Guettarda ac reana }}{\text { Krause }}$ & whole fruit & $x$ & & tree $-15 m$ & $\begin{array}{l}\text { h. mount. sav. } \\
\text { on } g r a n i t e\end{array}$ & 106 & 50 & D & & & & 000 & 00 & & & & & & \\
\hline$\frac{\text { Hilligillustris }}{\left(V_{e l l .}\right) \text { Schuriti: }}$ & flowers & - & - & $\begin{array}{l}\text { epiphytic } \\
\text { scrub in } \\
\text { tree crowns }\end{array}$ & High forest & - & - & & & & & & & & & & & & $\mathrm{f}$ \\
\hline $\begin{array}{l}\text { RUTACEAE } \\
\frac{\text { Zanthoxylum rhoifolium }}{\text { Lam. }}\end{array}$ & youn $h_{h}$ letres & - & - & tree $-15 \mathrm{~m}$ & high forest & $1^{*}$ & $0.3^{*}$ & & & & & & & & & & 1 & 11 & \\
\hline \multicolumn{20}{|l|}{ SAPINDACEAE } \\
\hline Paultimiasp. & $\begin{array}{l}\text { Whole lruit } \\
\text { young leave: }\end{array}$ & $\mathrm{x}$ & & liana & high forest & - & - & & & & & & & & & & $\begin{array}{ll}0 & 0 \\
1 & 1\end{array}$ & & \\
\hline$\frac{\text { Paullinia acuminata }}{\text { Uitt. }}$ & $a \leq 11$ & $x$ & & Iiana & bigh futent & - & - & & 00 & & & & & & & & & & \\
\hline$\frac{\text { Paullinia sphaerocarpa }}{\text { L.C. Rich. ex. Juss. }}$ & 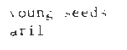 & $x$ & $x$ & $1 i d 5 . t$ & $\begin{array}{l}\text { ti.th turets, } \\
\text { theriverb.f. }\end{array}$ & - & - & 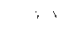 & i & 00 & & & & & & & & & $2 y$ \\
\hline$\frac{\text { Paullinia spicata }}{\text { Benth. }}$ & aril & $x$ & & lianis & $\begin{array}{l}\text { b. . ts firrestiesp. } \\
\text { it prina-weiny }\end{array}$ & $3^{*}$ & $1^{*}$ & - & 0 & & & 0 & 。 & & & & & & \\
\hline$\frac{\text { Paullinia tricornis }}{\text { Radik. }}$ & aril & $x$ & & li in & hich torent & $2^{\mathbf{t}}$ & $1^{*}$ & - & & & & & & 0 & $\therefore 0$ & & & & \\
\hline$\frac{\text { Telisia squatiosa }}{\text { Rad/k. }}$ & mesucarp & x & & tree - . 4: & thet 10res $=5$ & - & - & & & & & & & & & & & o & \\
\hline
\end{tabular}


FAHILY / Species

\section{SAPOT ACENE}

Achrouteris ponifera

Chrysophyllum, auratura

$\frac{\text { EccLInusa guianensis }}{\text { Eyma }}$

\section{Peleurrellasp.}

$\frac{\text { Micropholis guyanensis }}{\text { (A.DC.) Plerre }}$

$\frac{\text { Pouteria guianensis }}{\text { Eyma }}$

Pouteria sp.

SIKAROUBACEAT

Quassie simarouba

\section{TERCULLIACEAR}

Guszuma uLmifolia$$
\text { Lam. }
$$

$\frac{\text { Sterculia excelsa }}{\text { Mart. }}$

STYRACACEAE

Styrax aff. fanshawei Sandw. et micrasterus Perkins

MENDONCIACEAE Mendonc 10 hof fmannsegglans
Nees

\section{TIL IACEAE}

$\frac{\text { Apeiba echinata }}{\text { Gaertn. }}$

Apeiba Rlabra

Apeiba schomburgkif

Apeliba tibourbou $\begin{array}{llll}\text { parts eaten } & \begin{array}{l}\text { seed } \\ \text { dispersal }\end{array} & \text { seed } & \text { predation } \\ \text { of platit }\end{array}$

$\begin{array}{ll}\text { forest type } & \begin{array}{l}\text { of food } \\ \text { plants }\end{array}\end{array}$

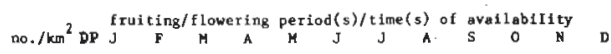

tree $-30 \mathrm{~m}$

h.mount, sav. f., 19

high forest

$9-$

$\circ \circ 00$

mesocsip

mesocarp mesocarp

me socarp

tree - 30m high forest

mesocarp

young seeds me socarp

me socarp

betk

whole fruit/

nesocarp

flowers

whole fruit/

mesocatp

me socarp

f lowers

mesocarp

me socatp

me socarp

mesocarp tree $-25 m$

h.mourt. sav, f.

tree $-25 \mathrm{~m}$

h, mouat. sav.f. 363

on granite +

tree $-40 \mathrm{~m}$ high forest

tree $-30 \mathrm{~m}$ high forest

$0.5^{\star} 0$ 。

$173 \quad \square 0$

66

0

$x \quad$ tree $-30 \mathrm{~m}$ high forest 15

tree $-25 \mathrm{~m} \mathrm{high} \mathrm{forest}$

tree - 33m hIgh forest

155

tree $-40 \mathrm{~m}$ h1gh forest

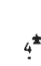

$74-$

$\circ \quad \circ$

$4-$

7 -

$25-00$ tree $-30 \mathrm{~m}$ high forest 53 $\circ 00.0$

$\circ \circ 00$

$\circ 000000$

$\circ 0$

(a) 
TA 12 I / species

\begin{tabular}{|c|}
\hline $\begin{array}{l}\text { ULMACEAE } \\
\frac{\text { Ampelocera edentula }}{\text { Kuhlmann }}\end{array}$ \\
\hline $\begin{array}{l}\text { VERBENACEAE } \\
\frac{\text { vitex stahelii }}{\text { Holdenke }}\end{array}$ \\
\hline $\begin{array}{l}\text { VIOLACEAE } \\
\frac{\text { Leonia glycycarpa }}{\text { R, et P. }}\end{array}$ \\
\hline $\begin{array}{l}\text { VOCHYSTACEAE } \\
\frac{\text { Quelea diniziz }}{\text { Ducke }}\end{array}$ \\
\hline HONEY \\
\hline $\begin{array}{l}\text { INSECTA } \\
\text { Lepidoptera }\end{array}$ \\
\hline Isoptera \\
\hline $\begin{array}{l}\frac{\text { Hymenopter }}{\text { Larvae of pollinating and }} \\
\text { parasitic wasps in figs } \\
\text { of different species } \\
\text { (fam. Agaonidae) }\end{array}$ \\
\hline
\end{tabular}

parts eaton $\begin{aligned} & \text { sead } \\ & \text { dispersal }\end{aligned} \begin{aligned} & \text { seed } \\ & \text { predation of plant forest type of pood }\end{aligned}$

tree $-20 \mathrm{~m}$ high forest

young leaves

flowers

whole fruit/

mesocarp

mesocarp

Elowers

$\mathrm{x}$

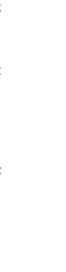

\begin{tabular}{|c|c|c|c|c|}
\hline tree $-45 m$ & $\begin{array}{l}\text { high forest, } \\
\text { h, mount. sav.f., } \\
\text { esp. edges of } \\
\text { liane forest }\end{array}$ & 72 & 34 & - \\
\hline tree $-20 \mathrm{~m}$ & $\begin{array}{l}\text { high forest, } \\
\text { esp in swampy } \\
\text { places }\end{array}$ & $1^{\star}$ & $0.5^{\star}$ & - \\
\hline tree $-40 \mathrm{~m}$ & high forest & 50 & 24 & - \\
\hline
\end{tabular}

tree -40 m high forest $50 \quad 24-$
in high high forest
trees
on trunks high fotest
trees
in $f$ igs

$\begin{array}{lll}1 & 1 & \text { I } \\ & & \mathrm{f}\end{array}$

$\circ 00$

6 $f \in$ 
Table II. The 58 plant families providing spider monkeys with food during the present study are rarked according to the number of species eaten. For each family the percentage of the total number of feeding records is also given. The three subfamilies of the Leguminosae are counted as families, and the family Guttilerae is taken sensu lato.

\begin{tabular}{|c|c|c|c|c|c|}
\hline \multirow{2}{*}{$\begin{array}{l}\text { Plant family } \\
\text { Moraceoe }\end{array}$} & \multirow{2}{*}{ food } & \multirow{2}{*}{ 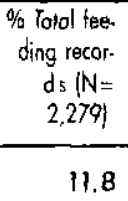 } & \multicolumn{2}{|c|}{ 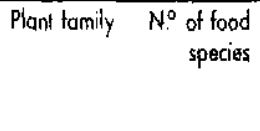 } & \multirow{2}{*}{$\begin{array}{r}\begin{array}{r}\text { \% Total feeding } \\
\text { records }(\mathrm{N} \text {. } \\
2,279)\end{array} \\
0.1 \\
02\end{array}$} \\
\hline & & & $\begin{array}{l}\text { Orchidacede } \\
\text { Sterculiacede }\end{array}$ & $\begin{array}{l}2 \\
2\end{array}$ & \\
\hline Bignoniaceas & 12 & 2.3 & Capparaceae & 1 & 1.0 \\
\hline Guttiferae & 9 & 2.1 & Caricacece & $i$ & 0.1 \\
\hline Caesalpiniaceae (Leg.) & 8 & 3.9 & Combretaceae & 1 & 0.05 \\
\hline Lecythidaceae & 7 & 1.8 & Dioscareacede & 1 & 0.05 \\
\hline Meliaceae & 7 & 5.8 & Flacourtiocede & 1 & 2.2 \\
\hline Papilionacede & 7 & 2.4 & Gesneriacede & 1 & 0.05 \\
\hline Sapotaceae & 7 & 8.6 & Gnetaceae & 1 & 0.2 \\
\hline Cellastraceae & 0 & 1.1 & Goupiaceoe & 1 & 0.05 \\
\hline Chrysobalanaceae & 6 & 1.7 & Humiriaceae & 1 & 0.9 \\
\hline Sapindacege & 6 & 0.9 & Loganiacede & 1 & 0.5 \\
\hline Annonaceoe & 5 & 3.6 & Loranthacede & 1 & 0.05 \\
\hline Araceae & 4 & 3.1 & Marcgraviaceae & 1 & 0.7 \\
\hline Burseraceae & 4 & 5.6 & Melastomataceae & 1 & 0.6 \\
\hline Convolvulacear & 4 & 1.1 & Menispermaceae & 1 & 0.6 \\
\hline Palmoe & 4 & 0.9 & Olacaceare & 1 & 0.3 \\
\hline Tiliacene & 4 & 1.1 & Passifloraceae & 1 & 0.05 \\
\hline Boraginaceoe & 3 & 1.7 & Piperaceas & 1 & 0.4 \\
\hline Cactacede & 3 & 1.0 & Polygalaceae & 1 & 0.5 \\
\hline Euphorbiaceae & 3 & 0.9 & Rutacede & 1 & 0.1 \\
\hline Lauracea $\theta$ & 3 & 0.4 & Simaroubaceae & 1 & 0.2 \\
\hline Rubiaceae & 3 & 0.5 & Styracaceae & 1 & 0.3 \\
\hline Anacardiaceae & 2 & 0.8 & Thunbergioceae & 1 & 0.1 \\
\hline Apocynoceae & 2 & 0.7 & Ulmaceae & 1 & 0.7 \\
\hline Bombocaceae & 2 & 1.8 & Verbenoceae & i & 0.6 \\
\hline Cucurbitaceae & 2 & 1.2 & Violacede & 1 & 0.0 .5 \\
\hline Icacinaceas & 2 & 0.3 & Vochysiaceae & 1 & 0.05 \\
\hline Myristicaceoe & 2 & 10.9 & Unidentified & 1 & 0.1 \\
\hline Myrtaceae & 2 & 0.1 & & & \\
\hline
\end{tabular}

4.1.1. Fruits. In total, spider monkeys were feeding on 171 kinds of truit. In addition to berries, drupes, pods and capsules, infructescences with the fruits adnate to the enlarged receptacle or to the fleshy perianth (as in several Moraceae) forming the major edible part, figs and compound fruits are also referred to as fruits. Fruits can be dehiscent or indehiscent, dry or pulpy; the outer layer can be thin, leathery or woody. Fruits consumed by spider monkeys ranged in size from the tiny globose drupes of Hyeronima laxiflora, $0.3-0.5 \mathrm{~cm}$ in diameter, to the woody, thick-walled, operculate fruits 
of Lecythis davisii tath grow to about $20 \mathrm{~cm}$ in diameter, or to the leathery elongate pods of Cedrelinga cateniformis that measure up to $80 \mathrm{~cm}$ in length. As a rule, spider monkeys didn't waste energy and time by selectively feeding on particular parts of truits, but instead swallowed whole fruits or, in the case of a thick or tough outer layer, bit open the fruit and swallowed the contents (pulp including seeds, or seed(s)/stone(s) surrounded by an edible layer of mesocarp, or with an edible aril attached).In many kinds of fruit the layer of mesocarp or the aril is strongly attached to the seed or stone (e.g., in all Burseraceae and Inga) and is therefore hard to separate. This may be an adaptation of the plant species to dispersal by specialized frugivorous birds and mammals such as Ateles. Most other Surinam monkey species, when feeding on truits of this kind, spit out the seed(s) or stone(s) after chewing or sucking off the edible part. In many cases they damage the seeds, spoiling them for germination. Spider monkeys swallowed whole fruits in $25.2 \%$ of total fruitfeeding records (39.3\% of total fruit species eaten). They dropped the outer layer and swallowed the mesocarp or aril (mostly including seeds or stones) in $68.2 \%$ of total fruit-feeding records $(44.7 \%$ of total fruit species eaten) (table V). Young seeds or parts of them (e.g., the plumula in Eperua) were consumed in $3.7 \%$ of total fruitfeeding records (13.7\% of total fruit species eaten). Fruits of some genera, such as Licania, Couepia and Gnetum, were eaten by scraping off the exo-and mesocarp c.q. perigonium, and dropping the seed/stone ( $1.5 \%$ of total fruit-feeding records; $4.2 \%$ of total fruit species eaten). Furthermore, spider monkeys fed exclusively on the exocarp in young fruits of Ceiba pentandra $(0.3 \% ; 0.6 \%)$, on the young columella of Cedrela odorata $(0.2 \% ; 0.6 \%)$, the pseudofruit (= fleshy pedicel) of Anacardium giganteum $(0.1 \% ; 0.6 \%$ ) and the juice of Geissospermum sericeum $(0.9 \% ; 0.6 \%)$, dropping the rest of the fruit.

It is important to distinguish between ingestion of seeds and digestion of seeds. Spider monkeys mostly swallow seeds intact with out mastication and the seeds pass through the digestive tract without losing their ability to germinate. In addition, seeds are sometimes dropped after the softer edible parts have been removed from them with the hands or mouth. On the other hand, seeds of certain families (e.g., Lecythidaceae) are eaten in an 
Table III. The most important plant genera providing the spider monkeys with food, ranked accoording to the percentages of the total number of feeding records.

\begin{tabular}{lcl}
\hline Genus (Family) & $\begin{array}{c}\text { No. of } \\
\text { species }\end{array}$ & $\begin{array}{l}\text { \% Total feeding } \\
\text { records (N=2,287) }\end{array}$ \\
\hline Virola (Myristicaceae) & 2 & 10.9 \\
Inga (Mimosaceae) & 12 & 9.5 \\
Ecclinuso (Sapotaceae) & 2 & 5.5 \\
Guarea (Meliaceae) & 2 & 4.3 \\
Tetragastris (Burseraceoe) & 2 & 3.7 \\
Cecropia (Moraceae) & 2 & 3.2 \\
Dimorphandra (Caesalpiniacece) & 1 & 3.1 \\
Philodendron (Araceae) & 3 & 3.0 \\
Bagassa (Moraceae) & 1 & 2.8 \\
Achrouteria (Sapotoceae) & 1 & 2.6 \\
Laetia (Flacourtioceae) & 1 & 2.2 \\
\hline
\end{tabular}

unripe stage. After extracting these young seeds from the fruit, they are chewed into a mealy pulp and digested. Such behavior is referred to as seed predation because the seeds ob. viously are destroyed. Swallowing seeds and excreting them intact results in seed dispersal (endozoochory). When seeds are removed from the fruit and dropped, this behavior is referred to as seed droping. When the monkeys drop seeds from the fruiting tree, these seeds are not dispersed any more than would be the case if they fell by themselves. However, in some cases the monkeys may carry fruits for considerable distances away from the source tree before eating them and dropping the seeds intact. This is a definite case of dispersal (exozoochory), but for the sake of convenience I include these rare cases under seed dropping.

Spider monkeys consumed fruits in a ripe stage in $96.0 \%$ of total fruit-feeding records ( 150 species; $N=1,902$ ). Seed dispersal took place in $93.5 \%$ of total fruit-feeding records (138 species), and seed dropping in $2.7 \%$ of total fruit-feeding records (10 species).Parts of young fruits were eaten only in $4.0 \%$ of total fruit-feeding records (24 species), whereas seed predation was recorded in $3.7 \%$ (23 species) (table VI). As shown in figure 19 , seed predation was recorded in $3.1 \%$, seed dispersal in $77.4 \%$ and seed dropping in $2.4 \%$ of all feeding records $(\mathrm{N}=2,287)$.

Seeds dispersed by spider monkeys ranged from the tiny seedlets of many Moraceae (e.g. Bagassa, Cecropia, Coussapoa, Ficus) to the large ellipsoid seeds of Platonia insignis (Guttiferae), which measure up to $4 \times 2 \mathrm{~cm}$. 


\begin{tabular}{|c|c|c|c|c|c|c|c|c|c|}
\hline Rank & Species & $A$ & B & $\mathrm{C}$ & Rank & Species & $\AA$ & $\mathrm{B}$ & $\mathrm{C}$ \\
\hline 1 & Virola melinonii & 243 & 233 & 10,7 & 30 & Carapa procera & 20 & 19 & 0.9 \\
\hline 2 & Guarea grandifolia & 91 & 72 & 4.0 & 31 & Ampelocera edentula & 19 & 19 & 0.8 \\
\hline 3 & Ecclinusa guianensis & 76 & 76 & 3.3 & 32 & Pachira msignis & 19 & 18 & 0.8 \\
\hline 4 & Cocropia sciadophylla & 32 & 71 & 3.2 & 33 & Apeiba echinata & 18 & 13 & 0.8 \\
\hline 5 & Dimorphandra sullei & 70 & 64 & 3.1 & 34 & Clusia scrobiculata & 17 & 15 & 0.7 \\
\hline 6 & Bagassa guianensis & 63 & 59 & 2.8 & 35 & Spondias mombin & 17 & 14 & 0.7 \\
\hline 7 & Inga odulis & 60 & 60 & 2.6 & 36 & Norantea guianensis & 17 & 13 & 0.7 \\
\hline 8 & Achrouteria pomifera & 59 & 59 & 2.6 & 37 & Geissospermum sericeum & 16 & 16 & 0.7 \\
\hline 9 & Inga alba & 57 & 37 & 2.5 & 38 & Licania micrantha & 16 & 15 & 0.7 \\
\hline 10 & Tetragastris panamensis & 56 & 48 & 2.5 & 39 & Brosimum parinarioides & 16 & 13 & 0.7 \\
\hline 11 & Laetia procera & 50 & so & 2.2 & 40 & Brosimum lactescens & 16 & 12 & 0.7 \\
\hline 12 & prieurella sp. & 50 & 49 & 2.2 & 41 & Protium neglectum & 16 & 8 & 0.7 \\
\hline 13 & Philodendron scandens & 39 & 34 & 1.7 & 42 & Dicranostyles guianensis & 15 & 9 & 0.6 \\
\hline 14 & Vataireopsis specioea & 36 & 36 & 1.6 & 43 & Hylocereus sp. a & 14 & 14 & 0.6 \\
\hline 15 & Ephodranthls guiamensis & 34 & 33 & 1.4 & 44 & Vitex stahelii & 14 & 14 & 0.6 \\
\hline 16 & Guatteria chrysopetala & 32 & 32 & 1.4 & 45 & Abuta grandifolia & 14 & 13 & 0.6 \\
\hline 17 & Cordia lomatoloba & 29 & 29 & 1.2 & 46 & Bellucia grossularioides & 14 & 13 & 0.6 \\
\hline 18 & Tetragastris altissima & 29 & 24 & 1.2 & 47 & Inga cf capitata & 14 & 12 & 0.6 \\
\hline 19 & Inga bourgoni & 27 & 27 & 1.1 & 48 & Inga a acroceyhola & 14 & 11 & 0.6 \\
\hline 20 & Philodendron acutatum & 26 & 25 & 1.1 & 49 & Adenocalymna sp. & 13 & 13 & 0.6 \\
\hline 21 & Protium polybotryum & 26 & 19 & 1.1 & 50 & Licania majuscula & 13 & 13 & 0.6 \\
\hline 22 & Clarisia racemosa & 24 & 24 & 1.0 & 51 & Maytenus sp. & 13 & 13 & 0.6 \\
\hline 23 & Couratari stellata & 23 & 21 & 1.0 & 52 & Coussapaa latifolia & 13 & 9 & 0.6 \\
\hline 24 & Ceiba pentandra & 22 & 22 & 1.0 & 53 & Inga cinnamomea & 12 & 12 & 0.5 \\
\hline 25 & Ahedia macrophylla & 22 & 20 & 1.0 & 54 & Moutabea guianensis & 12 & 11 & 0.5 \\
\hline 26 & Inga leiocalycina & 22 & 13 & 1.0 & 56 & Pouroma mollis & 11 & 2 & 0.5 \\
\hline 22 & Capparis maroniensis & 22 & 6 & 1.0 & 57 & Arrabidaea corallina & 10 & 10 & 0.4 \\
\hline 28 & Cayaponia ophthalmica & 21 & 20 & 0.9 & 58 & Hyeronima laxiflora & 10 & 10 & 0.4 \\
\hline 29 & Sacoglottis cydonioides & 21 & 13 & 0.9 & 59 & Eperua falcata & 10 & 9 & 0.4 \\
\hline
\end{tabular}


Trichilia quadrijuga/surinamensis Maripa scandens

Pterocarpus officinalis

Helicostylis tomentosa

Anomocteriun granulosum

Ficus gardneriana

Guettarda acreana

Peperomia glabella

Attalea maripa

Condia sagotii

Guarea kunthiana

Unonopsis glaucopetala

Euterpe oleracea

Cayaponia rigida

Duguetia sp.

Minquartia guianensis

Piptadenia suaveolens

Parinari excelsa

Pereskia aculeata

Styrax aff fanshawei et micrasterus

Eschweilera poiteaui

Loretia cordata

Xylophragma seemannianum

Amplizoma sp.

Drypetes variabilis

Helicostylis pedunculata

Ocotea wachenheimii

Parkia nitida

Parkia pendula

Paulinia acuminata

Pouteria 8p. n. 400

Virola surinamensis

\begin{tabular}{ll} 
& 0.4 \\
& 0.4 \\
& 0.4 \\
& 0.4 \\
& 0.3 \\
& 0.3 \\
& 0.3 \\
& 0.3 \\
& 0.3 \\
& 0.3 \\
& 0.3 \\
& 0.3 \\
\hline & 0.3 \\
\hline & 0.3 \\
\hline & 0.3 \\
\hline & 0.3 \\
6 & 0.3 \\
\hline 6 & 0.3 \\
\hline 6 & 0.3 \\
\hline 5 & 0.3 \\
\hline 5 & 0.3 \\
\hline 5 & 0.3 \\
\hline 5 & 0.2 \\
5 & 0.2 \\
\hline 5 & 0.2 \\
\hline 5 & 0.2 \\
\hline 5 & 0.2 \\
\hline 5 & 0.2 \\
\hline & 0.2 \\
\hline & 0.2 \\
\hline & 0.2 \\
\hline
\end{tabular}

0.4

0.4

0.3

0.3

3

0.3

0.3

0.3

3

.2

0.2

2

2

2

0.2
Paullinia spicata

Tabebuia serratitolia

Honey

Quassia simarouba

Apeiba glabra

Gustavia bexapetala

Hylocereus sp. b

Inga pezizifera

100 Inga rubiginosa

101 Oenocarpus bacaba

102 Gnetum urens

103 Paullinia sphaerocarpa

104 Dialium quianense

105 Eschweilera carrugata

106 Couepia caryophylloides

107 Alchorneopsis floribunda

108 Caterpillars (2 species)

109 Cedrela odorata

110 Cheiloclinium podostemmum

111 Clusia grandiflora

112 Maquira guianensis

113 Perebea mollis

114 Heterópsis jenmani

115 Pouteria guianensis

116 Termites

117 Dipteryx odorata

118 Stizophyllum inaequilaterum

119 Hymenolobium petraeum

120 Mendoncia holfmannseggiana

121 Pithecellobium jupunba

122 Paullinia tricomis

123 Pourouma minor 


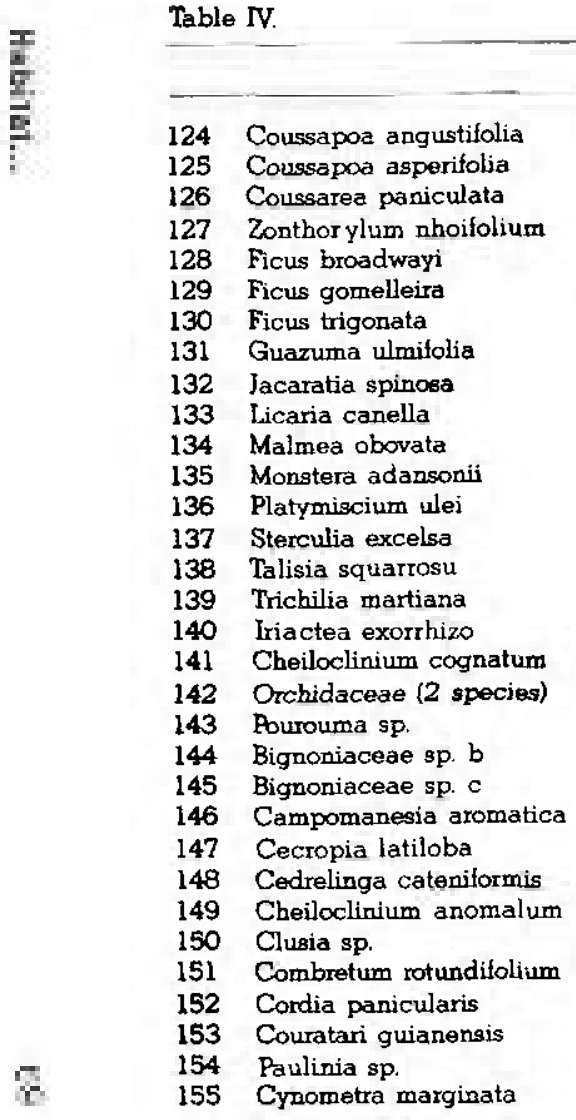

156 Dioclea macrocarpa

157 Dioscorea trifida

158 Enterolobium schomburgki

160 Euterpe precatoria

161 Ficus guianensis

162 Ficus citrifolia

163 Ficus malacocarpa

164 Ficus nymphaeifolia

165 Ficus pertusa

166 Ficus trigona

167 Gesneriaceae (1 species)

168 Hillia illustris

169 Inga stipularis

170 Leonia glycycarpa

171 Vantarria parvifloro

172 Licania densiflora

173 Loranthaceae (1 species)

174 Maripa glabra

175 Tanaecium nocturnum

176 Nectandra kunthiana

177 Operculina hamiltoni

178 Parahancornia fiscianlata

179 Parinari campestris

180 Platonia insignis

181 Qualea dinizii

182 Sympbonia globulifera

183 Trymatococcus oligandrus

184 Trymatococcus paraensis

185 Anacardium giganteum

186 Apeiba schomburgkii

187 Apeiba tibourbou
159 Eschweilera congestiflora

0.05

0.05

0.05

0.05

0.05

0.05

0.05

0.05

0.05

0.05

0.05

0.05

0.05

0.05

0.05

0.05

0.05

0.05

0.05

0.05

0.05

0.05

0.05

0.05

0.05

0.05

0.05

0.05

0.05

0.05

0.05

10.05

10.05

0.05 
188 Bignoniaceae sp.a

189 Cheiloclinium hippocrateoides

190 Chrysophyllum auratum

191 Clusia platystigma

192 Clusia purpurea

193 Copaifera epunctata

194 Copaifera guianensis

195 Eperua rubiginosa

196 Eugenia feyoi

197 Ficus insipida
198 Goupia glabra

199 Inga coriacea

200 Inga thibaudiana

201 Lecythis davisii

202 Micropholis guyanensis

203 Ormosia paraensis

204 Passiflora glandulosa

205 Peltogyne yenosa

206 Rheedia benthamiana

207 Tabebuia capitata
$0 \quad 0.05$

$0 \quad 0.05$

$0 \quad 0.05$

$0 \quad 0.05$

$0 \quad 0.05$

$0 \quad 0.05$

$\begin{array}{ll}0 & 0.05\end{array}$

$0 \quad 0.05$

$0 \quad 0.05$

$\begin{array}{ll}0 & 0.05\end{array}$ 
Table V. Different food items eaten by Ateles p. paniscus, expressed as: (A) - percentage of total number of fruit species eaten for any part of fruit, $(B)$ - percentage of total number of food species eaten for any part of food plant, (C) percentage of total number of fruit-feeding records for any part of truit, and (D) - percentage of total number of teeding records tor any part of food plant and other food.

\begin{tabular}{|c|c|c|c|c|c|}
\hline Parts eaten & pecies & $\begin{array}{r}\text { A } \\
\text { Total Fruit } \\
\text { species } \\
(N=17 i)\end{array}$ & $\begin{array}{r}\text { B } \\
\text { \%Totel food } \\
\text { species } \\
{[N=207]}\end{array}$ & $\begin{array}{r}C \\
\% \text { Total huit } \\
\text { (N=eding records } \\
(\mathcal{N}=\{, 895)\end{array}$ & $\begin{array}{r}\text { D } \\
\text { \% Totol feeding } \\
\text { records } \\
(N=2,287\}\end{array}$ \\
\hline Whate fruittinfrud. fig swallowed & 66 & 39.3 & 31.9 & 25.2 & 20.9 \\
\hline Moscxarp (axo torp dinpped, seed (s) ingasted) & 50 & 29.8 & 24.1 & 43.8 & 36.3 \\
\hline Aril (excocorp dropped, seed(s) ingested) & 25 & 14.9 & 12.0 & 24.4 & 20.2 \\
\hline Young sood(s)/plumula \pm young aril & 23 & 13.7 & 11.1 & 3.7 & 3.1 \\
\hline Exa-and mesocorp/pericorp/perigonisum (seeds dropped) & 7 & 4.2 & 3.3 & 1.5 & 1.3 \\
\hline Exocarp (rest of fruit droppod) & 1 & 0.6 & 0.5 & 0.3 & 0.2 \\
\hline Juice (chewed fruil dropped) & 1 & 0.6 & 0.5 & 0.9 & 07 \\
\hline $\begin{array}{l}\text { Columella + young seeds. } \\
\text { Pseudofruit (fleshy pedicel) (truit dropped) }\end{array}$ & 1 & 0.6 & 05 & 0.1 & 0.05 \\
\hline Flowersinflorescencosithorol buds & .48 & & 16.0 & & 6.4 \\
\hline Young leavestathoots/petioles & $28:$ & & 13.5 & & 7,9 \\
\hline Pseudobulbs (flesthy thickened stems of certain orchids) & 2 & & 1.0 & & 0.1 \\
\hline Autiat roots & 2 & & 1.0 & & 0.2 \\
\hline Bark & 11 & & 5.3 & & 1.7 \\
\hline Decoying woodirotien shouths of Altoloo ragio & 1 & & 0.5 & & 0,3 \\
\hline Honey & $=$ & & - & & 0.2 \\
\hline Coterpillars & $i$ & & - & & 0.1 \\
\hline Termites & I & & - & & 0.1 \\
\hline
\end{tabular}

Table VI. Seed dispersal, seed predation and seed dropping in Ateles p. paniscus expressed as the percentage of total number of truit species and as the percentage of total number of fruit-feeding records.

\begin{tabular}{llll}
\hline Category & $\begin{array}{l}\text { No. of fruit } \\
\text { species }\end{array}$ & $\begin{array}{l}\text { \% Total fruit } \\
\text { species } \\
(\mathrm{N}=171)\end{array}$ & $\begin{array}{l}\text { \% Total truit-feeding } \\
\text { records } \\
(\mathrm{N}=1,902)\end{array}$ \\
\hline Seed dispersal & 138 & 80.2 & 93,5 \\
Seed predation & 23 & 13.5 & $\overline{3} .7$ \\
Seed dropping & 10 & 5.8 & 2.7 \\
\hline
\end{tabular}

Generally, the faeces of spider monkeys which are dropped out of a tree, contain a number of differents kinds of seed and fall apart because of a lack of binding substance. The seeds and stones are widely spread over the forest floor. Within a few minutes, all kinds of dung beetles arrive, attracted by the smell, and bury both faeces and seeds under the soil surface. Seeds and stones dispersed in'this way by spider monkeys usually do not attract terrestrial seed predators and therefore have a fair chance of germinating successfully. In contrast, many fruiting trees used by the monkeys attract 
terrestrial seed predators such as agoutis, acouchis, pacas, deer and peccaries when their fruits fall in quantity.

Also, other animals such as bruchid weevils (Bruchidae), several terrestrial birds, and tortoises are attracted by the fruits dropped from these tres. In particular curassows ( $C r a x$ alector) and trumpeters ( $P_{s o}$ phia crepitans) appear to be attracted by crashing sounds and vocalizations of Ateles, and frequently feed on the soft parts of fruits accidently dropped by the monkeys. By analyzing stomach contents of many curassows and trumpeters, it appeared that all seeds and stones ingested had been more or less damaged by either the bill or by grinding (Van Roosmalen and Mittermeier, in prep.). Consequently, these birds do not act as dispersers of larger seeds. Analysis of many dung samples of tortoises (Geochelone spp.) living in the area showed that these animals can act as seed disperser at least for several plant species (e.g., Anacardium giganteum, Tetragastris spp., Protium spp., Spondias mombin, Cayaponia spp., $E_{C-}$ clinusa guianensis). However, in contrast to Ateles, these animals swallow only small amounts of fruits and their daily travel distance is small.

Ateles p. paniscus appeared to be an important seed disperser for many plant species and it seemed to be the only dispersal agent for several species that were not eaten by specialized frugivorous birds (e.g., cotingids, trogons, toucans and guans), Alouatta or Geochelone. In cases where seed predation by other monkeys, tree porcupines, insects, birds and terrestrial animals is strong, the plant's dispersal may be completely dependent on Ateles. Most obviously, this was the case for the following species: Capparis maroniensis, Sacoglottis cydonioides, Gustavia hexapetala, Dimorphandra pullei, Guarea grandifolia, Guarea kunthiana, Trichilia spp., Brosimum parinarioides, Clarisia racemosa, Minquartia guianensis, and Ecclinusa spp. This phenomenon is demonstrated nicely by fruiting Brosimum parinarioides (Moraceae), a widely dispersed tree that is not common in the Voltzberg study area (table I). First of all, Chiropotes satanas, an important seed predator (Van Roosmalen, Mittermejer and Milton, 1981), visited a particular tree regularly over several months, feeding on the young seeds. Macaws (Ara spp.) and squimels (Sciurus, Sciurillus) also occasionally fed on the young seeds. Many maturing fruits were seen still hanging in the tree. Except for 
Ateles, which swallowed the fruits whole, other monkeys such as Saimiri sciureus, Cebus apella and Cebus nigrivittatus were seen feeding on the pulp and dropping the mature seeds. Fruiting was abundant and many fruits fell down spontaneously, especially when monkeys moved through the crown. When Ateles or other monkeys fed on the ripe fruits, several terrestrial seed predators (e.g., agoutis (Dasyprocta), acouchis (Myoprocta), deer (Mazama) and sometimes peccaries (Tayassu))could be seen coming into the area to feed on the falling seeds. Curassows (Crax) and trumpeters ( $P_{\text {sophia) were }}$ also seen feeding on the pulp of fallen fruits and swallowing the seeds. At this time, hundreds of seeds could be seen still lying on the forest floor under the tree. However, the next day all the seeds were gone. All the mature seeds that were dropped on the forest floor were either eaten or buried by scatter-hoarding rodents (e.g., agoutis), which protect the seeds in this way against predation by bruchid weevils. In this particular case, the tree may depend completely on Ateles for its dispersal, especially since a single seed that germinates far from the parent plant will have much greater chances for survival. Seeds from forgotten stocks or ones opened too late by scatter-hoarders may all germinate, but these monospecific beds of seedlings may well be discovered by herbivores ( e.g., Mazama, Tapirus) which also forage for such edible seedling beds.

4.1.2. Flowers. Flowers contribute only a small portion to the spider monkey diet. In total, Ateles fed on 33 species of flower $(16 \%$ of total food plant species recorded) in $6.4 \%$ of total feeding records (table V; fig. 19). Of the 33 plant species producing edible flowers, 17 are trees, 13 lianes and three epiphytes. Ranked according to the percentage of total flower-feeding records, the most important family was Bignoniaceae (25.9\%; 11 species), followed by Meliaceae (17.7\%; two species), Marcgraviaceae (11.6\%; one species), Cactaceae (10.9\%; three species), Bombacaceae $(9.5 \%$; two species), Mimosaceae (6.8\%; four species), Ulmaceae ( $4.8 \%$; one species), Tiliaceae $(2.7 \%$; one species), Caesalpiniaceae $(2.0 \%$; one species), Guttiferae (1.4\%; two species), Papilionaceae (1.4\%; one species), and Moraceae, Rubiaceae, Sterculiaceae and Vochysiaceae $(0.7 \%$; one species each).

Most of the flowers were consumed when at the mature stage or at a stage just before opening. When small, the entire flower or 
inflorescence was swallowed. (e.g., Ampelocera edentula, Carapa procera, Enterolobium schomburgkii and Piptadenia suaveolens), but most flowers were only partly eaten. In particular corolla and/or tips of petals were preferred in many species (especially in Bignoniaceae), so that the calyx was dropped or the rest of the flower was not picked oft the peduncle, stem or twig. Several species were used only for the tips of stamens, style and/or for the stigma (e.g., Pachira insignis, Hylocereus spp., Pereskia aculeata and Parkia spp.), some for the sticky central body of staminodes (Clusia spp.), for the bracts transformed in honeycups (Norantea guianensis) or for the thickened perianth (as in pistillate inflorescences of Coussapoa spp.).

Ranked according to the percentage of total flower-feeding records, most important species were Guarea grandifolia (13.6\%), Norantea guianensis (11.6\%), Hylocereus sp. a. and Adenocalymna sp. $(8.8 \%)$, Pachira insignis (7.5\%), Arrabidaea corallina $(6.8 \%)$, Ampelocera edentula (4.8\%), Piptademia suaveolens (4.1\%), Carapa procera $(4.1 \%)$ and Xylophragma seemannianum $(3.4 \%)$. The other 23 species contributed less than $3.0 \%$ of total flower-feeding records.

4.1.3. Leaves. As flowers, leaves only contributed a small portion to the spider monkey diet. In total, Ateles fed on 28 species of leaf $(13.5 \%)$ of total food plant species recorded) in $7.9 \%$ of all feeding records (table $\mathrm{V}$; fig. 19). Of the 28 plant species producing edible leaves, 19 are trees, four lianes, one twiner and four epiphytes. Ranked according to the percentage of total leaf-feeding records, the most important family was Papilionaceae (29.7\%; four species), followed by Araceae (25.0\%; three species), Bombacaceae (12.2\%; two species), Meliaceae (5.8\%; one species), Bignoniaceae and Moraceae (4.7\%; two species), Piperaceae ( $4.7 \%$; one species), Caesalpiniaceae $(3.5 \%$; two species), Ulmaceae $(2,3 \%$; one species), Mimosaceae (1.2\%; one species), Lauraceae (1.2\%; one species), and Myristicaceae, Chrysobalanaceae, Rutaceae, Caricaceae, Sapindaceae, Dioscoreaceae, Gesneriaceae and Loranthaceae (0.6\%; one species each).

It has to be emphasized that spider monkeys fed exclusively on young or flush leaves during the present study. They were never seen eating a single mature leaf. In many cases only the apex of flush leaves was eaten ( e.g., Pachira insignis, , Ceiba pentandra, 
Jacaratia spinosa, Tabebuia serratifolia and Cecropia sciadophylla) or parts of the lamina were ripped off the costa with the mouth (e.g., Ceiba pentandra, Pachira insignis). In addition to flush leaves, young shoots and young leaves still rolled up in the sheath were also eaten (e.g., in Philodendron scandens and $P$. acutatum), and so was the base of the petiole (e.g., in Philodendron scandens and Carapa procera).

Ranked according to the percentege of total leaf-feeding records, the most important species were Vataireopsis speciosa and Philodendron scandens (both $20.9 \%$ ), followed by Ceiba pentandra $(8.1 \%)$, Carapa procera $(5.8 \%)$, Pterocarpus officinalis and $P$. vs. santalinoides $(5.2 \%)$, Peperomia glabella $(4.7 \%)$, Pachira insignis $(4.1 \%)$ and Philodendron acutatum (3.5\%). The other 20 species contributed less than $3.0 \%$ in total leaf-feeding records.

4.1.4. Pseudobulbs. Pseudobulbs are the thickened, fleshy stems of certain Orchidaceae, mostly epiphytes, that function as a food and water reservoir. Spider monkeys fed on parts of pseudobulbs, while dropping the leaves or part of the entire plant, of two epiphytic orchid species which were growing on boughs high up in tall trees. They contributed only $1.0 \%$ to the total food plant species recorded and only $0.1 \%$ to total feeding records (table V; fig. 19). Neither species could be identified from the collected sterile samples, but both seemed to be quite abundant in the study area.

4.1.5. Aerial roots. Aerial roots are the roots growing out of stems of certain plants that hang in the air. On reaching the ground, they may take hold in the forest soil and start to act as normal roots. Many tropical epiphytes produce aerial roots. Spider monkeys fed on the tips of aerial roots of two species of Araceae. To reach the tip of these pendent aerial roots, the monkeys had to pull up several meters of them. The two species of Philodendron contributed only $1.0 \%$ in total food plant species recorded and $0.2 \%$ in total feeding records (table $\mathrm{V}$; fig. 19).

4.1.6. Bark and decaying wood. In total, Ateles p. paniscus was seen feeding on the bark of 11 species $(5.3 \%$ of total food plant species recorded), in $1.7 \%$ of all feeding records (table V; fig. 19). All species were trees. Ranked according to the percentage of total bark-feeding records, the most important species was Licania micrantha (37.5\%), followed by Ingaalba (30.0\%), Sacoglottis cydo- 
nioides $(10.0 \%)$ and Inga leiocalycina $(5.0 \%)$, with seven other species contributing $2.5 \%$ each.

Spider monkeys seemed to prefer decaying bark. Since almost all trees used were healthy, the edges of scars, waterholes and certain patches along the underside of boughs were particularly selected. These patches, though not always easily reached, had a softer, more or less decaying texture resulting from rain water flowing out of holes in the bough or trunk.

Occasionally, and apparently only in some adults, spider monkeys were seen feeding on the rotten sheaths of previously shed leaves of the palm Attalea maripa, which were found under the crown just above the smooth trunk. In particular an old female several times entered low forest at the very edge of liane forest or "rock savanna", where this palm frequently occurs, to feed on this rotten palm wood. The material, which looked from a distance like peat, was consumed as if palatable and by the handful. This food was taken in only $0.3 \%$ of all feeding records (table V; fig. 19).

4.1.7. Honey. Occasionally, spider monkeys fed on honey from bees nests in tree holes. This occurred in only $0.2 \%$ of all feeding records (table V; fig. 19). The monkeys obtained honey by reaching with one hand into the hole and licking the honey off the fingertips. The holes were difficult to reach and the monkeys often had to climb down big trunks.

4.1.8. Insects. Spider monkeys were definitely seen feeding on insects in a few cases. At least two species of small caterpillars were eaten, and accounted for $0.1 \%$ of all feeding records. Both species were collected but were not identifiable. The caterpillars were found in clumps of several hundred, attached by silk to the leat. Spider monkeys were also seen eating one species of termite in $0.1 \%$ of all feeding records (table $V$; fig. 19). They usually opened the tunnels of termites that ran up the huge bole of an emergent tree, especially of the species Hymenolobium flavum, and waited until a number of termites walked into the open. They picked up the termites with their tongues and seemed to select only certain types of termites. Whether they selected workers or soldiers was not clear. Spider monkeys were never seen opening the termite nests that sometimes high occurred up in tree crowns. 
In addition to actively feeding on insects, spider monkeys accidently ingest minute insects such as the stages of pollinating and parasitic wasps that are present in all kinds of figs. However, Ficus species were rare and widely dispersed in the Voltzberg study area. In total, 11 species of fig were eaten, contributing only $1.0 \%$ in the total number of feeding records.

The animal-protein intake of Ateles p. paniscus is probably restricted to the above mentioned cases. It never fed on rotten fruits which usually contain animal life, and wormy fruits were dropped as well.

\subsection{Feeding Behavior}

This section deals with spider monkey daily activities, with special emphasis on feeding behavior. A typical day for the long wet season is compared with a typical day for the long dry season, sin. ce feeding behavior appears to be strikingly influenced by the seasons.

A typical spider monkey day during the long wet season. In Surinam, day dawns at $5.45 \mathrm{~h}$ local time. At first light, when it is still dark on the forest floor, a subgroup of spider monkeys moves out of its sleeping site for the past night, the huge, isolated crown of a $50 \mathrm{~m}$ tall Hymenolobium flavum at the foot of the Voltzberg. Just before they depart, the area beneath the crown is bombed by enormous amounts of excreta as the members of the subgroup urinate and defecate almost simultaneously. The old female with her son, a juvenile-3, is the first to move. The others, a female with her daughter, a juvenile-2, and an old male follow in a row. Soon, they all reach a group of food trees not far from the sleeping tree. Apparently, the proximity of these trees was one of the reasons for choosing the Hymenolobium as the past night's sleeping site. After feeding on four trees from $6.00-7.00 \mathrm{~h}$, without once feeding together at the same time on the same food tree, they leave and travel over one kilometer within 20 minutes. Throughout this travel, the old female determines the route taken and the activity patterns of the subgroup as a whole. Suddenly, the fomale with the juvenile-2 splits off. Apparently, she has in mind a different foraging route and other food sources than the old female. Within a short time, they are out of sight and we continue to follow the old female, her son and the old male. Before $8.00 \mathrm{~h}$, they feed together on two other trees. At $8.00 \mathrm{~h}$, the 
female starts to bark and is immediately followed by the male, resulting in a siamang-like barking duet. This call seems to act as a long distance contact call advertising the presence of this subgroup to other spider monkeys in the area. It differs considerably from the long call that only adult males give. After feeding on another tree, the monkeys rest for half an hour. After another feeding bout of 15 minutes in the same tree, they leave and move quickly over $500 \mathrm{~m}$. After feeding for a short time, they start another barking duet in which the juvenile tries to take part. They then feed again for 20 minutes in the same tree. Between $10.15-12.15 \mathrm{~h}$, the monkeys feed on ten different trees over a $500 \mathrm{~m}$ route that is still fully determined by the old female. After that, they rest for 40 minutes in the upper part of the canopy. The old female lies on her back with the eyes closed in a branches fork. After feeding another 15 minutes in a nearby tree, they rest again for half an hour. At 14.00 $h$, they move away and soon join another subgroup. This subgroup usually ranges in another "core area" within the home range inhabited by the group. It contains four animals: two females, a male juvenile 2 and a dependent infant-1(-2). A greeting ceremony follows. This is usually performed only by the males on such occasions but this time also involve the females who briefly embrace one another. The greeting completed, the juveniles start to play, whereas the adults rest for 45 minutes in an emergent tree. At the beginning of the rest period, the old male gives a long call, answering another male about $500 \mathrm{~m}$ away. At $14.45 \mathrm{~h}$, all seven animals move and the adults feed for 20 minutes on two nearby trees. The juveniles keep on playing. They then all travel in roughly the same direction from which the old female's subgroup came prior to uniting with the second subgroup. After briefly traveling and feeding, the other subgroup splits off at $16.00 \mathrm{~h}$, and moves away very rapidly in the direction of the long call heard just betore, the one given by the third male of the spider monkey study group. The old female's subgroup follows them for a short time but soon returns to its own route. At this point, they are in the same area as they were at $8.00 \mathrm{~h}$. After feeding on two trees, the old female's subgroup mo. ves back the way it just came, but then turns more to the north. Now, they seem to be in a hurry, traveling very fast for over 600 $\mathrm{m}$. I have a lot of trouble tracking them now. Another male is calling nearby and some minutes later a young male meets and joins 
the subgroup after a short greeting ceremony with the two males of the subgroup. Immediately after this, the two adult males start a barking duet. The newcomer then moves away and is followed by the other males. The old female, who has so far led the subgroup, stays back. She searches for new food sources, entering the lower part of the canopy here or the top of a tall tree there, and she checks several fruiting trees for stage of maturity. In the meantime, the males are rushing forward in the direction of the coming night's sleeping tree some $500 \mathrm{~m}$ away. Somewhat later, the old female follows at a distance of about $50 \mathrm{~m}$. After feeding together on one tree at $17.15 \mathrm{~h}$, all four travel toward the sleeping tree, the female in tront. They feed on another four trees in the direct neighbourhood of the sleeping tree until $18.00 \mathrm{~h}$. For part of the time, the juvenile and the young male playtight and chase each other. From $18.00-18.30$ $h$, they feed on another three trees next to the large buttressed sleeping tree, a Piptademia suaveolens. At $18.30 \mathrm{~h}$, the young male moves away to another sleeping tree. Ten minutes later, the old male and the juvenile enter the sleeping tree by crossing a gap between two crowns, swaying back and forth, grasping the extreme twigs of a crownlet of the sleeping tree, and sweeping across. Some minutes later, the female enters the tree in the same way and all three feed for a while on the inflorescences of the sleeping tree itself and on the young leaves of two epiphytes (Araceae) hanging down along the huge bole of the tree. At $18.40 \mathrm{~h}$, when it is almost dark on the forest floor, the monkeys prepare for the night, each in its own site. The juvenile rests against his mothers belly for a while, then moves to his own site some $6 \mathrm{~m}$ away from her. When I finally reach the camp, it is completely dark.

Today, the three animals observed continuously have fed on a total of 38 different food plants belonging to 13 species, of which two were very important: Virola melinonii, of which 17 different trees were used, and Laetia procera, of which six different trees were used. A total of 32 feeding records was made for fruits (not including the data from faeces), four ír flowers and two for young leaves. Total feeding time was $5 \frac{1}{2}$ hours, total resting time between $6.00-18.45$ $\mathrm{h}$ was three hours, and total travel distance was $4,500 \mathrm{~m}$.

A typical spider monkey day during the long dry season. When I reach the night's sleeping tree at $6.00 \mathrm{~h}$, the sky is clear. The spi- 
der monkeys are still resting high up in the crown of a $50 \mathrm{~m}$ tall Vochysia tomentosa. The crown is completely isolated from the canopy beneath and difficult to enter. This subgroup consists of an old female and her juvenile son about three years old, two of the same animals as followed in the previous example, and a second adult female. At about $6.30 \mathrm{~h}$, all animals defecate. The faeces are compact, containing mainly vegetable matter and a few seeds of only one species. Sitting side by side, the mother leans over her son and nurses him for two minutes. Although over three years old, he is still nursed from time to time. Within one or two months he will be weaned, and shortly after this his mother will come into estrus for the first time in about four years. At $6.45 \mathrm{~h}$, the second female descends to the canopy by leaping across a gap. Mother and son keep on resting. Twenty minutes later, they also move out of the sleeping tree and join the other female some $120 \mathrm{~m}$ away, where she was waiting for them and uttering soft contact calls. Together, they travel about $100 \mathrm{~m}$ toward a fruiting tree of the most important food species of the moment, Dimorphandra pullei, which offers edible pods for four to seven months. For the next 40 minutes, they feed on the old, desiccated mesocarp of these fruits by ripping open the woody pods longitudinally. No infructescence or pod is spoiled and dropped before it has been eaten out completely. Especially in this time of food scarcity, spider monkeys tend to eat very economically. At $8.00 \mathrm{~h}$, all three travel back over $100 \mathrm{~m}$. Then the second female splits off while mother and son are feeding on young leaves of a low tree. Following this, the mother and son rest in the middle part of the canopy for about two consecutive hours. At $10.15 \mathrm{~h}$, they feed briefly on young leaves of Pachira insignis. From 10.25 10.35 and $10.45-10.55 \mathrm{~h}$, they feed on the fruits of another $\mathrm{Di}^{-}$ morphandra pullei. After a short travel bout, they eat the flowers of Guarea grandifolia for 26 minutes, broken by a rest of 15 minutes. From 11.45 - 12.15 h, mother and son travel slowly and feed on a few flowers of Bignoniaceae lianes. From $12.15-13.10$ $h$, both animals rest high up in an emergent tree. When they continue on their way, they meet the other female again. Some greeting vocalizations are heard, then all three feed on young leaves of Pachira insignis, a common tree in this region which is restricted to pina swamp forest along creeks. For one hour they feed without interruption on these young leaves by tearing the apical part of the 
leaflets off the costa by their teeth. From 14.15 - $15.45 \mathrm{~h}$, all three rest, the second female in a nearby tree. From $15.45 \mathrm{~h}$ on, they feed continuously for 40 minutes on the flowers of a Guarea grandifolia tree. After a rest of 20 minutes, mother and son travel to the same sleeping tree as used the previous night and enter the crown by leaping. The second female stays back, and feeds on the flowers of a nearby Guarea grandifolia for another ten minutes. She enters the sleeping tree at $17.00 \mathrm{~h}$. She sits down at the same place as the previous night, five meters above mother and son, who are sitting side by side. The juvenile is then nursed for five minutes while he is lying between his mothers knees. The time now is $17.15 \mathrm{~h}$ and the monkeys are ready to sleep, lying in forks of branches in the late afternoon sun.

Today, neither long calls nor barking duets were heard and no other subgroups were encountered. The observed animals ted on a total of 14 different food plants belonging to 10 species, of which, three; were very important: Dimorphandra pullei, two fruiting trees; Guarea grandifolia, three flowering trees; Pachira insignis, young leaves of two trees. Not including the data from faeces, a total of three feeding records were made for fruits, five for flowers and six for young leaves. Total feeding time was $3 \frac{1}{2}$ hours of which three hours were spent on the three most important species. Total resting time between $6.00-18.45 \mathrm{~h}$ was seven hours, and total travel distance was $700 \mathrm{~m}$.

Comparing these two days which are typical for each season, the following differences are most striking. Day range length during the long wet season can be more than six times day range length during the long dry season. During the long wet season, total resting time is much less and total feeding time is much more. Also, the sleeping tree is left much earlier and entered much later in the wet season than in the dry season. The diet in the dry season includes a much higher percentage of flowers and young leaves than during the wet season. In the dry season, the monkeys feed very economically and activity is kept to a minimum. In general, subgroups are smaller during the long dry season and fewer encounters take place with other subgroups. The monkeys are also more silent.

4.2.1. Foraging techniques. In general, individual feeding activity within a subgroup was closely synchronized. When feeding 
for prolonged periods of time on the same food source before traveling to another, the first feeding bout was usually similar for all members of the subgroup and consisted of eating at high speed. Later, short resting bouts could alternate with short feeding bouts, which did not invariably occur simultaneously for all subgroup members involved. Juveniles spent less time feeding than adults, spending the extra time playing either alone or with other juveniles and/or adults.

Usually, the daily itineraries and activity pattems of a subgroup were determined by a so-called leading female or sometimes shared by two leading females. The latter occurred quite rarely and only over short periods of time. Each female seemed to have somewhat different foraging routes in mind, resulting in frequent fission and fusion. The route connecting the principal food plants was the shortest one possible, both in geographical and ecostructural sense, and the leading female usually did not hesitate. It appeared that she had roughly mapped out in her mind the foraging route for a particular day early in the morning or perhaps even the day before. Another observation which may help in interpreting this remarkable behavioral pattern is the fact that a leading female that temporarily joins another subgroup led by another female and following a completely different route, could not readily relocate her planned route after splitting off. For this reason, she engaged in some apparently inappropriate behavior such as retracing earlier travel routes and revisiting certain food sources, or traveling back and forth.

When a foraging subgroup was relatively large (four or more independently locomoting animals), the monkeys tended to spread their attention over different food sources at any given point in time, thus avoiding possible agonistic interactions. This type of foraging seems to be possible only when food supply is abundant and one or more other food sources are likely to be nearby. During the long wet season, the average subgroup size observed was therefore larger.

Spider monkeys live in medium-size, loosely associated groups which fragment into subgroups of varying size and composition, and roam independently in the same general area. The only persistent association is that of a female and her offspring up to four years of age. All other members of the group will join a subgroup only 
temporarily and then switch to another or go their own way. Temporary, solitaries are frequently observed, especially during the long dry season.

A leading female appeared to know a certain part of the home range the best and this area is referred to as her "core area". Leading females were often observed in their "core areas" checking fruit sources that were soon to become available. On a number of such occasions a leading female was seen going off the route in order to check a food source with unripe fruit, testing the fruit, dropping it and then retuming to the rest of the subgroup. Some days, this behavior was observed especially during the late aftemoon, when the rest of the subgroup was playing or traveling ahead towards the sleeping tree. The behavior was not performed every day but occurred at least every fourth day. In this way important food plants which were approaching the edible stage could be incorporated at the right moment into the female's pre-planned foraging route. If this interpretation of foraging behavior is correct, the knowledge of a leading female becomes most impressive, especially since the phenological picture is constantly changing and a foraging route involves a very large number of plants dispersed over a wide area.

The unusual social structure of spider monkeys seems to be a highly efficient adaptation to exploiting available mature-fruit sources, especially since other group members can rapidly learn about food by conspecific cueing (Slatkin and Kiester, 1974). Since mature-fruit sources are patchily distributed and the fruits can only be obtained for short periods of time and in relatively small amounts, the optimal foraging strategy seems to be a core-area system of leading fermales, with whom other group members may join temporarily to exchange knowledge on available food sources.

4.2.2. Feeding techniques. In general, fruits are found at or near the periphery of tree crowns. Spider monkeys tend to feed by suspension and are seldom seen picking a fruit and carrying it to a bough or branch before consuming it. Fruits are picked with one hand. Many fruits are swallowed whole, but when the outer layer is tough the fruit is normally opened with the teeth and then eaten. Few manipulations were observed when spider monkeys fed on truit. This may be related to lack of a functional thumb. When truits are small or compound, spider monkeys may eat them by removing 
part or all of the infructescence, holding it with the hand (s) and snapping oft the fruitlets with the mouth (eg., in Alchomeopsis, Hyeronima, Pourouma, Oenocarpus and Guettarda). Some fruits are only chewed for the juice (e.g., in Geissospermum) or for the soft outer layer which is scraped off with the teeth (e.g., in Couepia, Licania and Ceiba). Manipulation of fruit parts with particular fingers was rarely observed. Pulp and seedlets of Clusia grandiflora are scraped out of the locules with two fingers. Pods are ripped open allong the suture (s) with the teeth and eaten out, while both valves are held in the hands (e.g., in Inga). Bivalved fruits dehiscing at full maturity, such as those of Virola, are bitten just before opening, while the fruit is held with both hands. At this mature stage, the fruit is easily opened by applying pressure. After pulling the valves apart with the hands or with hands and teeth, the monkey swallows both stone and aril. When feeding on young seeds, spider monkeys bite out part of the truit-wall so that the seed(s) can be removed for eating.

To reach some food items, such as the infructescences of Philodendron scandens and the aerial-root tips of Philodendron, spider monkeys pull up several meters of stem or aerial root while sitting on a branch. Once a spider monkey was seen sitting on a bough and looking down along a bunch of hanging stems of a Philodendron scandens. It first pulled up the wrong stem and, then the right one to obtain the fruiting spadix. Breaking off branches to feed on their infructescences, a common behavior of capuchin monkeys, was observed very rarely and seemed to occur rather by accident than by intent.

Leaves selected as food were always young leaves and usually ocurred at the periphery of the tree crown. Leaves would be eaten either directly or after they were torn off twig or stem. Selective feeding on particular leaf parts was observed in many cases. Parts of the lamina were ripped off the costa with the teeth, or only the apex of the leaf was bitten off. Young shoots and leaves still rolled up in the sheath were also eaten, and sometimes the base of the petiole was preferred.

Flowers occur mostly at the periphery of crowns and usually were eaten directly by pulling the flowering twig or stem towards the mouth or by picking the flowers themselves with one hand. In 
many cases only the corolla was bitten off and eaten, the rest of the flower remaining attached to the peduncle, stem or twig. Sometimes, all or part of the inflorescence was broken off with the hand before it was eaten. Some flower species were selected only for the tips of stamens, style and/or stigma, others for the sticky central body of staminodes, the honeycup or the thickened perianth.

Bark was eaten by biting off pieces with the teeth, chewing for a while and ingesting the pulpy mass. The bark eaten was usually decaying or rotten. Most of the trees from which bark was selected were apparently healthy. However, in certain places, such as the edges of scars and water-filled holes, the bark was more or less decaying because of continuous contact with rain water. Frequently, places on the underside of boughs which were obviously affected by regularly flowing rain water were chosen. These places were often hard for the monkeys to reach and so required much effort. Inconvenient postures had to be adopted such as hanging by all five extremities under a thick, horizontal bough. Many of these particular patches appeared to have been used for several years and their exact location was apparently known from earlier visits. It is worth noting that of 11 species of tree providing a source of bark for the spider monkeys in the study area, 10 species provided the monkeys with another food item as well (eight for fruit, two for flush leaves). Only Nectandra kunthiana provided only bark, but it was not observed fruiting during the study period and might well provide the monkeys with edible fruits at certain times. Selective feeding on bark may be important for the monkeys, since several tree species are known to have poisonous bark (e.g., Vataireopsis speciosa). Restricting barkeating to well-known species may be advantageous as it may limit the chance of error. Also, use of the same bark feeding sites over a long period of time may help in avoiding dangerous errors, especially if barks (as opposite to fruits and flowers) are as difficult to differentiate for the monkeys as they were for the observer.

The method of drinking from tree holes resembles very much the drinking behavior of slamangs and gibbons as described by Chivers (1974) and Ellefson (1974). Water-filled holes are usually found at the junction of branches or places where a branch or bough is broken off and the wood is rotten. These holes are filled with water most of the year and play an important role as a water source, es- 
pecially during the long dry season. The monkey dips its hand into the hole, raises it rapidly to the mouth, lifting up the elbow in order to suck the water running off the back of the hand. This action is sometimes repeated over 80 times in a row. Water was seen being taken in the same manner from the beakers formed by the tightly imbricate leaf bases of big Bromeliads growing epiphytically on boughs. During the long wet season, drinking was observed rarely as the juicy fruits which make up the main part of the diet apparently contain enough water.

4.2.3. Feeding heights. Ateles is primarily an animal of the upper levels of the forest. Its main foods, nutritious large-seeded fruits, are usually found in the upper periphery of canopy crowns. Of a total of 6,105 feeding minutes, recorded for 30 days evenly spread over the year, $77.2 \%$ was spent in the upper levels of the forest. $22.8 \%$ of this time was spent in emergents ( $30 \mathrm{~m}$ or more) and $54.4 \%$ in the upper part of the camopy $(25-30 \mathrm{~m})$. The lower levels of the canopy and the understory were relatively unimportant, with $16.4 \%$ spent in the middle part $(20-25 \mathrm{~m})$ and $5.6 \%$ in the lower part of the canopy $(15-20 \mathrm{~m})$. Ateles was rarely seen feeding in the understory $(3.15 \mathrm{~m})$, this stratum accounting for only $0.8 \%$ of total feedling time.

4.2.4. General feeding postures. Locomotion and postural behavior of Ateles p. paniscus has been studied in depth by Mittermeier (1978) in the Voltzberg study area. Some of the results concerning locomotion during feeding and general feeding postures will be mentioned briefly here. For detailed description refer to Mittermeier (1978).

Climbing, especially "horizontal climbing", appeared to be the most important locomotion pattern during feeding. It is typefied by irregular limb use. Most locomotion takes place on twigs and branches, with twigs playing a greater role during feeding than during travel. Twigs are the most important supports in feeding postures since most of the feeding activities take place at the periphery of tree crowns. Feeding postures can be divided into three major categories: sitting, standing and suspensory. Sitting and standing postures are above branch, with the animal's weight on the support. Suspensory postures are those in which the major support is above or to the side. Most often they involve three limbs as support, the 
tail couting as a limb, in addition to the limb used to grasp food. Sitting and suspensory postures were the most important, while bipedal, tripedal and quadrupedal standing postures contributed $10-15 \%$. A vertical clinging posture is occasionally used, and on rare occasions Ateles will feed in a reclining position.

4.2.5. Food selectivit. In general, daily itineraries appeared to be made up of direct lines between subsequent sleeping trees, and were determined by several trees, lianas or groups of trees which were important in providing the monkey's food at that time. Spider monkeys were rarely seen visiting a particular food plant more than once a day. Only when the same sleeping tree was used on consecutive nights were some food sources, especially those occurring near this sleeping tree, used a second time.

Ripe fruits were usually selected from a distance by sight. Sometimes, as in the case of Sacoglottis cydonioides, Brosimum parinarioides and Clarisia racemosa, the monkey would inspect the truit by sniffing or biting it softly, since the extemal properties of these fruits do not give a definite indication of the stage of maturity.

Affected and wormy fruits were often recognized without being opened, even when they were marked only by very inconspicuous insect holes. There is also evidence for the selective feeding on tas. tier fruits from different trees of the same species. Form example, spider monkeys fed only on some of the simultaneously fruiting trees of the species Spondias mombin. The selected trees turned out, without exception, to produce sweet-tasting truits, whereas the neglected ones carried bitter-tasting fruits. Given the fact that Ateles paniscus was found one of the main dispersal agents of the large seeds of Spondias mombin, in this way it may provide selective pressure for sweet-tasting flesh in this species.

4.2.6. Temporal patterning of food choice. Spider monkey day ranges appeared to be determined mainly by certain food sources, here referred to as primary food sources. These sources usually produce large, relativel y fast-ripening (or in the case of leaves, ra. pidly flushing) crops of relatively nutritious food items, enough for at least three independently locomoting members of a subgroup to feed on together. Normally, teeding bouts on foods in this category are relatively long. Most of the species are available every year, but crops can tail during some years. Examples of primary food sources 
are: Ceiba, Protium, Tetragastris, Guarea, Virola, Achrouteria and Ecclinusa.

Secondary food sources are those producing smaller, slowripening crops of relatively nutritious foods, available for prolonged periods but at any moment offering a meal to only one or two independently locomoting animals. Examples are: Duguetia, Monstera (infructescence), Philodendron (infructescence), Hylocereus (inflorescence, infructescence), Jacaratia, Clusia, Leretia, Strychnos, Cecropia, Passiflora, Moutabea and Paullinia. These food sources will be used only by one or two members of a subgroup on its way from one primary food source to another. Normally, feeding bouts are short.

Tertiary food sources are defined as reliable sources mostly available for prolonged periods of time, sometimes even for several months or longer, providing relatively low-nutrient secondary food. These sources usually occur throughout the home range and may be used either by the whole subgroup or by members of a subgroup on their way from one primary food source to another. Feeding bouts vary in length, but may be quite long. Examples of tertiary food sources are: Philodendron (leaves, shoots and aerial roots), Licania (bark), Eperua (leaves), Pterocarpus (leaves), Norantea (flowers), Bellucia (fruits), Carapa (leaves), Cecropia (leaves) and Peperomia (shoots). Crops of tertiary food sources never fail and the monkeys can depend on them every year.

As an introduction to the materials on variation in food choice, I present outlines of food choice and foraging routes performed by a spider monkey subgroup during four consecutive days in both December 1977 and April 1978. These months are chosen for comparison because of strikingly different availability of preferred food. Also, during both periods of observation, a particular leading female and her juvenile son were observed continuously, and were found to determine fully the ranging behavior of all individuals joining ther subgroup during the observation period. In tables VII and VIII, all food species and food items used by a soider monkey subgroup during four consecutive days in both December 1977 and April 1978 are listed, as are the amount of feeding time for each food item and species and the category to which each food source belongs. The corresponding daily itineraries of the subgroup are mapped in figures 20 and 21 . 
During four consecutive days of observation in December, primary food sources accounted for $87 \%$ of total feeding time, whereas feeding on secondary and tertiary food sources made up only $2 \%$ and $11 \%$ respectively. During four consecutive days of observation in April, primary food sources accounted for $86 \%$ of total feeding time, whereas feeding on secondary and tertiary food sources only made up $12 \%$ and $2 \%$ respectively.

4.2.7. Diurnal variation in food choice. Spider monkeys appeared to select for variety of food. As shown in tables VII and VIII, a spider monkey subgroup used on average 14 different food items each day in December and in April. These items represented only about a quarter of the total monthly number of food items used. Among these, three or four food items were considered most important based on the amount of feeding time and the estimated total weight of food ingested. Duration of the feeding visit to these three or four primary food sources could be long but never exceeded two hours. Most feeding visits to other food sources were short, ranging between one and 15 minutes.

Daily patterns of food use could be distinguished. Fruits were eaten throughout the day, but there were usually two feeding peaks: early in the morning and late in the afternoon. A third peak might occur around midday (between 11.00-13.00 h) (Van Roosmalen, in prep.). Leaf-eating occurred throughout the day in the period JulyJanuary and increased during the course of the day. From Janiary to July, however, it mainly occurred in the late afternoon. This pattern may be explained as follows. Fruit-eating requires more energy expenditure for suspensory behavior in the periphery of crowns than does leaf-eating. When fruits are abundant (from January to July) and activity budgets correspondingly high, it seems likely that the monkeys prefer to leave the easy activity of eating young leaves for the late afternoon when they are more tired. On the other hand, increase of leaf-eating in spider monkeys is correlated with decrease in extent and rate of ranging (for example, see table VII, December 5). Feeding on flowers occurred throughout the day in the period July-January, and increased during the course of the day in January and February. Flowers of Bignoniaceae and Cactaceae (e.g., Hylocereus), however, were especially consumed in the moming when the corolla has just opened. Feeding on bark and rotten palm sheaths 
was more frequently observed during the moming, with a peak between 10.00-12.00 h. The consumption of other food items, such as honey, insects, aerial roots and pseudobulbs occurred infrequently throughout the day, and the sample size is too small for determining general patterns.

4.2.8 Variation in food choice between days. Diet changed considerably from day to day. Spider monkeys usually exploited the food sources of a particular part of the home range in a two to fourday cycle. The cycling pattern shifted constantly at variable rate, depending on the kind and the availability of present food sources. In general, primary food sources, which are most responsible for determining the cycling pattern, are ripening or flushing over relatively short periods of time. Some of these sources provide an entire subgroup enough to feed on day after day. If not exploited by the monkeys, the mature fruits or infructescences either open their valves and become available to birds (e.g., Tetragastris, Laetia, Virola) or drop to the ground (e.g., Bagassa, Brosimum). Consequently, when such a preferred food source becomes available, a leading spider monkey female will include it in her itinerary almost every day (table VII, e.g., Bagassa guianensis and Brosimum parinarioides; table VIII, e.g., Tetragastris altissima and T. panamensis). In this way, these particular food sources may play a central role in daily ranging behavior for some time. Most primary food sources, however, neither have dehiscent fruit nor do they drop a truit crop in a short period of abundance. Rather, they produce mature fruit more gradually. Food sources of the latter kind may be visited once every two to four days (e.g., Spondias, Inga, Styrax). In the meantime, enough fruits can ripen and become available for the entire subgroup to feed on at a next visit. Solitary ranging animals may visit some of these sources in the interval between visits of a larger subgroup led by a dominant female, but the relatively small amount of food eaten by these animals probably does not affect the ranging pattern. In general, secondary food sources, which usually ripen slowly, are not visited more than once every two days. Most intervals between subsequent visits appeared to be longer than this, often ranging from three to eight days or more, depending on the food species. Tertiary food sources usually produce enough for short feeding visits every day, but since most 
Table VII. Food choice and teeding time for a spider monkey subgroup during four consecutive days in December 1977. The food sources listed correspond with those mapped in figure 20; (I) stands lor primary, (II) for secondary, and (III) lor tertiary food sources.

\begin{tabular}{|c|c|c|c|}
\hline Species & Food item & $\begin{array}{l}\text { Food } \\
\text { category }\end{array}$ & $\begin{array}{l}\text { Foedin } \\
\text { time (min }\end{array}$ \\
\hline \multicolumn{4}{|l|}{ December 2} \\
\hline 1. Bagassa guianensis & infructescense & I & 74 \\
\hline 2. Brosimum parinanoides & infructescense & I & 217 \\
\hline $\begin{array}{l}\text { 3. Inga pezizirera } \\
\text { 4. Philodendron aculatum }\end{array}$ & $\begin{array}{l}\text { pods checked only } \\
\text { y. leaves, shoots }\end{array}$ & III & 3 \\
\hline Sa. Philodendron scandens & shoots & III & 4 \\
\hline b. Coussapoa latifolia & infructescense & I & 4 \\
\hline 6a. Philodendron aculatum & aerial-root tips & III & 3 \\
\hline $\begin{array}{l}\text { b. Pauljinia sphaerocarpa } \\
\text { 7. Inga sp. }\end{array}$ & $\begin{array}{l}\text { y. seeds } \\
\text { pods checked only }\end{array}$ & II & 1 \\
\hline 8a. Eschweilera congestiflorá & y. seeds + aril & II & 3 \\
\hline b. Hillia illustris & flowers & III & 2 \\
\hline 9. Philadendron acutatum & aerial-root tips & III & 2 \\
\hline 10. Sacoglottis cydonioides & bark & III & 2 \\
\hline 11. Inga alba & bark & III & 2 \\
\hline 12a. Gesneriaceae indet. & $\mathrm{y}$. leaves & III & 11 \\
\hline b. Tabebuia serratifolia & $\gamma$ leaves & 琶 & 12 \\
\hline 13. Achrouteria pomifera & truit & I & 41 \\
\hline 14. Couratari stellata & bark & 前 & 2 \\
\hline 15. Ceiba pentandra & $y$. leaves & I & g \\
\hline
\end{tabular}

Weather: sunny: heavy rain from 14.36-15.00 and 17.00.19.00 h

Observation time: $5.48-17.00 \mathrm{~h}$

Day range size: $1.500 \mathrm{~m}$

Total food species used: 15

Total food items used: 18

Total feeding time 291 minutes (244 on primary, 4 on secondary and 43 on tertiary tood sources)

Most important food species calculated in feeding time: Brosimum parinarioides (117 mun.). Bagassa guianensis (74 min.). Achrouteria pomifera (4l min.)

Subgroup size and composition: $5.48-13.38 \mathrm{~h}-4\left(2 \% q 16, \mathrm{l}_{\mathrm{j}} 3 \delta^{\circ}\right)$

$13.38-17.00 \mathrm{~b} \cdot 7(+2 \mathrm{go}, \mathrm{II})$

\section{December 3}

1. Cecropia sciadophylla

2. Cecropia sciadophylla

3. Bagassa guianensis

4. Pouteria sp. (400)

5. Vataireopsis speciosa

6. Bagassa guianensis

7 . Peperomia glabella

b. Ficus guianensis

c. Ceiba pentandra

8. Xylophragma

infructescence
infructescence
infructescense
y. seeds
y. leaves
infructescence
shoots
figs
y. leaves
flowers

5.

Weather: sunny; heavy rain from $11.25-12.00$ and $12.07-12.35 \mathrm{~h}$

Observation time: $5.40 .13 .00 \mathrm{~h}$, then lost contact 
Table VII (Continued)

\begin{tabular}{llll}
\hline Species & Food item & $\begin{array}{l}\text { Food } \\
\text { category }\end{array}$ & $\begin{array}{l}\text { Feeding } \\
\text { Time (min. }\end{array}$ \\
\hline
\end{tabular}

\section{December 3}

Day range size: $900 \mathrm{~m}(+1,600 \mathrm{~m}$ bypothetic)

Total feeding time: 147 minutes (134 on primary, 7 on secondary and 6 on tertiary food sources)

Most important food species calculated in feeding time: Ficus guianensis (94 min.), Bagassa guianersis ( $26 \mathrm{~min}$.)

Subgroup size and compasition: $5.40-8.50 \mathrm{~h}-4\left(2,16,133 \mathrm{f}^{\mathrm{t}}\right)$

$8.50-13.00 \mathrm{~h}-3$ (lo left subgroup)

\section{December 4}

1. Bagassa guianensis

2. Brosimum parinarioides

3. Bellucia grossularioides

4. Ampelocera edentula

5. Sacoglottis cydonioides

6. Trichilja quadrijuga

7. Dimorphandra pullei

8. Vantanea

9. Achrouteria pomilera

10. Xylophragma seemannuanum

11. Coussapoa asperitolia

12. Cordia sagotti

13. Achrouteria pomileza

14. Clarisia racemosa

15. Ceiba pentandra

16. Inga leiocalycina

17. Guatteria chrysopetala

18. Achrouteria pomifera

19. Dimorphandra pullei

14. Clarisia racemosa

20. Licaria canella

21. Apeiba $\oplus$ chinata

22. Xylophragma seemanniasum

infructescense
infructescence
fruit
y. leaves
bark
fruit
frut
truit
fruit
flowers
infructescence
fruit
fruit
truit
y. leaves
bark
fruit
fruit
fruit
truit
y. leaves
flowers
flowers

$\begin{array}{lr}\text { I } & 29 \\ \text { I } & 32 \\ \text { III } & 11 \\ \text { III } & 1 \\ \text { III } & 1 \\ \text { I } & 18 \\ \text { I } & 10 \\ \text { II } & 5 \\ \text { I } & 10 \\ \text { III } & 8 \\ \text { II } & 5 \\ \text { I } & 5 \\ \text { I } & 4 \\ \text { I } & 5 \\ \text { I } & 37 \\ \text { III } & 5 \\ \text { I. } & 12 \\ \text { I } & 6 \\ \text { I } & 9 \\ \text { I } & 6 \\ \text { III } & 4 \\ \text { III } & 1 \\ \text { III } & 4\end{array}$

Weather: sunny; heavy rain from $16.45 \cdot 19.30 \mathrm{~h}$

Observation time: $5.40-16.45 \mathrm{~h}$

Day range size: $1,250 \mathrm{~m}$

Total food species used: 18

Total tood items used: 18

Total feoding time: 227 minutes (185 on primary, 7 on secondary and 35 on tertiary food sources)

Most important food species calculated in feeding time: Ceiba pentandra(37 min.), Brosimum parinarioides ( $32 \mathrm{~min}$ ), Bagassa guianensis (29 min.)

Achrouteria pomifera (20 min.), Dimorphandta pullei (19 min.)

Trichilia quadrijuga (I8 min.)

Subgroup size and composition: $5.40-12.15$ h -6 (30\%, 10, 1520, 1538 )

$12,15-16.45 \mathrm{~h}-3(2 \mathrm{q}, 1 \mathrm{~J} 2 \mathrm{O})$ 


\begin{tabular}{|c|c|c|c|}
\hline Species & Food itern & $\begin{array}{l}\text { Food } \\
\text { category }\end{array}$ & $\begin{array}{l}\text { Feeding } \\
\text { Time (min.) }\end{array}$ \\
\hline \multicolumn{4}{|l|}{ December 5} \\
\hline $\begin{array}{l}\text { 1. Vataireopsis speciosa } \\
\text { 2. Ceiba pentandra } \\
\text { 3. Achrouteria pomifera } \\
\text { 4. Achrouteria pomifera } \\
\text { 5. Bagassa guianensis } \\
\text { 6. Ampelocera edentula } \\
\text { 7. Bellucia grossularioides } \\
\text { 8. Bagassa guianensis }\end{array}$ & $\begin{array}{l}\text { y. leaves } \\
\text { y. leaves } \\
\text { fruit } \\
\text { fruit } \\
\text { infructescence } \\
\text { y. leaves } \\
\text { fruit } \\
\text { infructescense }\end{array}$ & II & $\begin{array}{c}53 \\
41 \\
35 \\
18 \\
16 \\
2 \\
10 \\
36\end{array}$ \\
\hline
\end{tabular}

Weather: sunny; heavy rain trom $11.35-11.50,13.48-14.37$ and $17.15-18.45 \mathrm{~h}$

Observation time: $5.45 .17 .10 \mathrm{~h}$

Day range size: $850 \mathrm{~m}$

Total food species used: 6

Total food items used: 6

Total feeding time: 210 minutes (198 on primary, $O$ on secondary and 12 on tertiary food sources)

Most important food species calculated in feeding time: Vataireopsis speciosa ( $55 \mathrm{~min}$.), Achrowteria pomifera (51 min.), Bagassa guianensis (51 min.), Ceiba pentandra (41 min.)

Subgroup size and composition: 3 (10, 10; 1130)

of these food sources are not really preferred by the monkeys and occur throughout the home range, they do not significantly affect daily ranging pattem. However, some tertiary food sources may be important, particularly during the long dry season when fruits are scarce.

Leading females each tend to exploit different parts of the home range (core areas), except when a fruit excess occurs. This pattern is shown in tables VII and VIII. Only two females belonging to the part of the group followed in December and April were leading females. Both were accompanied by a juvenile and were leading a subgroup consisting mainly of a male and/or one or more females with or without offspring. During the observation time in December, which is a period of relative scarcity of preferred fruits, both leading females rarely joined subgroups and obviously exploited different parts of the group's range. In April, both females trequently joined subgroups and then followed roughly similar itineraries, while feeding on somewhat different food sources. This pattern was made possible by the excess of preferred fruit in this period of the year. 


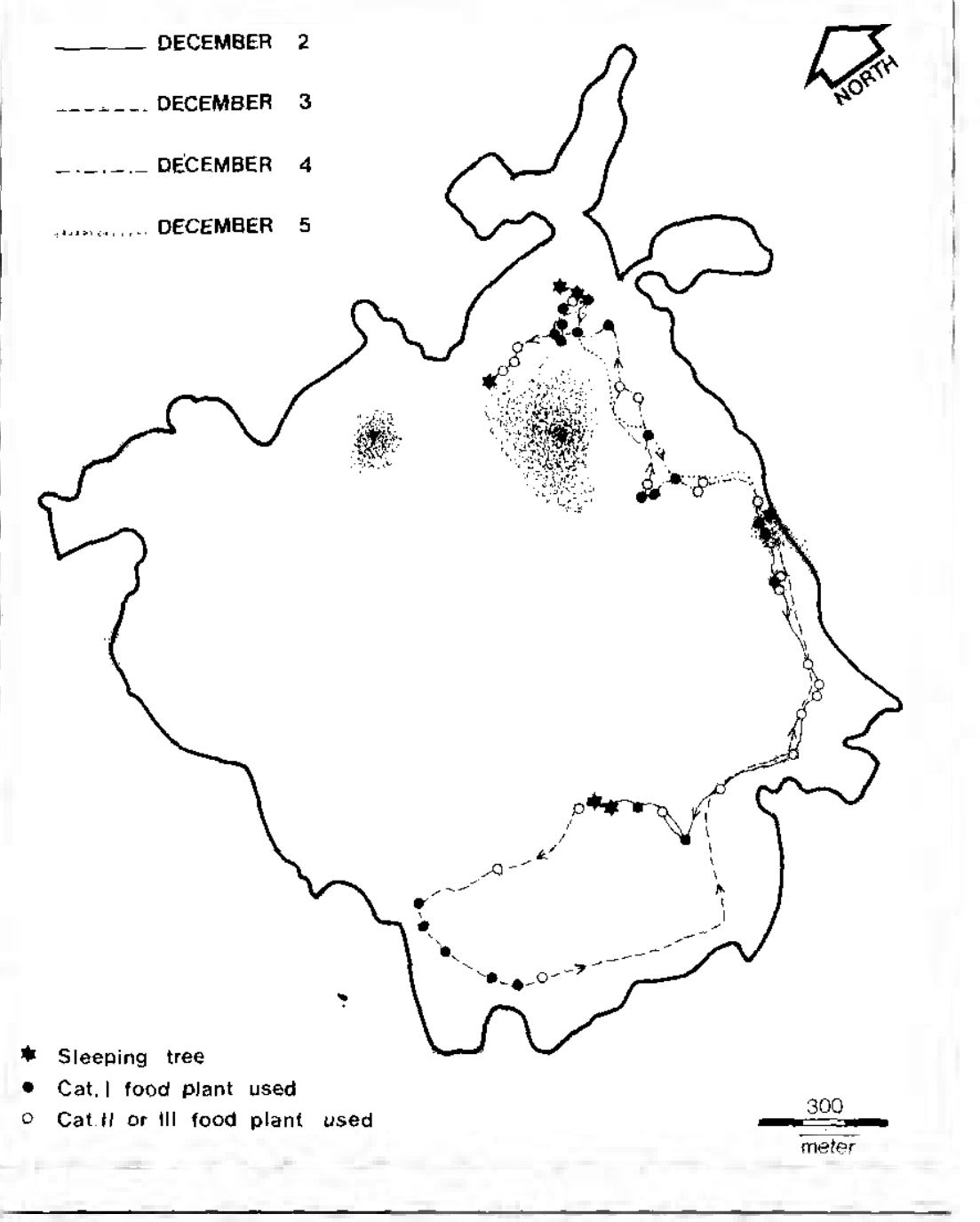

Figure 20 Routes followed by a spider monkey subgroup led by a certain female on four consecutive days in December 1977. The food sources used are indicated and correspond with those given in table VII In this figure, I only indicate whether a food source belongs to category I or to category II or III. 
Table VIII. Food choice and feeding time for a spider monkey subgroup during four consecutive days in April 1978. The food sources listed correspond with thoee mapped in figure 21; (I) stands for primary, (II) for secondary, and (III) for tertiary food sources.

\begin{tabular}{|c|c|c|c|}
\hline Species & Food item & $\begin{array}{l}\text { Food } \\
\text { category }\end{array}$ & $\begin{array}{r}\text { Feeding } \\
\text { time }(\min .)\end{array}$ \\
\hline \multicolumn{4}{|l|}{ April 23 } \\
\hline 1. Tetragastris altissima & fruit & I & 9 \\
\hline 2. Tetragastris panamensis & fruit & I & 2,5 \\
\hline 3. Tetragastris panamensis & fruit & I & 39: \\
\hline 4. Styrax of. tanshawei & fruit & 1 & $28:$ \\
\hline 5. Cecropia sciadophylla & infructescence & II & 14 \\
\hline 6. Ecclinusa sp. & fruit & I & 4 \\
\hline 7. Cayaponia ophthalmica & fruit & II & 2 \\
\hline 8. Guettarda acreana & fruit & iI & 4 \\
\hline 9. Sterculia excelsa & y. seeds & II & $2:$ \\
\hline 10. Cecropia sciadophylla & infructescence & II & $\mathfrak{I}$ \\
\hline 1I. Coussarea paniculata & fruit & I & 1 \\
\hline 12. Geissospermum sericeum & fruit (juice) & 1 & 7 \\
\hline 13. Rheedia macrophylla & fruit & 廿 & 7 \\
\hline 14. Guarea grandifolia & fruit & 1 & : \\
\hline 15. Geissospermum sericeum & fruit (juice) & I & $\mathrm{s}$ \\
\hline 16. Indet. (liana) & y. leaves & III: & \$ \\
\hline 17. Prieurella sp. & fruit & 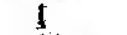 & 10 \\
\hline 18. Termites & termites & iit. & 4 \\
\hline 19. Cecropia sciadophylla & infructescence & II & 6 \\
\hline 20. Philodendron acutatum & y. leaves & III & 2 \\
\hline
\end{tabular}

Weather: sunny; no rain

Observation time: $10.25-18.20 \mathrm{~h}$

Day range size: $1,900 \mathrm{~m}$

Total food species used: 15

Total food items used: 15

Total feeding time: 183 minutes ( 136 on primary, 37 on secondary and 10 on tertiary food sources)

Most important food species calculated in leeding time: Tetragastris panamensis (64 min.), Styrax aff tanshawei (28 min.), Tetragastris altissima (25 min.), Cecropia sciadophyl. la (21 min.)

Subgroup size and composition: 10.25-17.00 h - 5 (3og, 1J3\%,111) after $17.00 \mathrm{~h}-6$ (lo joined subgroup)

$\begin{array}{ll}\text { April } 24 & \\ \text { 1. Tetragastris altissima } & \text { fruit } \\ \text { 2. Clusia scrobiculata } & \text { truit } \\ \text { 3. Tetragastris panamensis } & \text { fruit } \\ \text { 4. Tetragastris panamensis } & \text { fruit } \\ \text { 5. Guarea grandifolia } & \text { fruit } \\ \text { 6. Mendoncia hoffmannseggiana } & \text { fruit } \\ \text { 7. Guarea grandifolia } & \text { fruit } \\ \text { 8. Celastraceae indet. } & \text { fruit } \\ \text { 9. Cayaponia rigida } & \text { fruit } \\ \text { 10. Guarea grandifolia } & \text { fruit } \\ \text { 11. Guarea grandifolia } & \text { fruit }\end{array}$

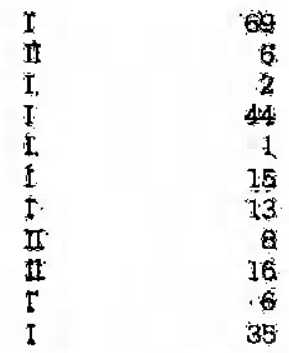


Table VIII (Continued)

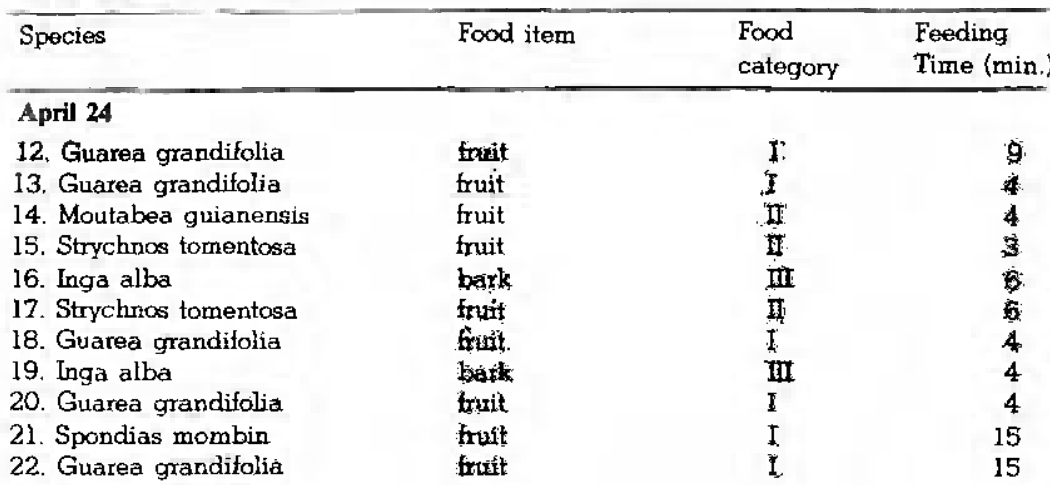

Weather: sunny; no rain

Observation time: $5.40-18.10 \mathrm{~h}$

Day range size: $2,680 \mathrm{~m}$

Total tood species used: 11

Total food items used: 11

Total feeding time: 289 minutes (236 on primary, 43 on secondary and 10 on tertiary tood sources)

Most important food species calculated in feeding time: Guarea grandifolia (91 $\mathrm{min}$ ), Tetragastris altissima (69 min.), Tetragastris panamensis (46 min.)

Subgroup size and composition: 5 (2og $10^{*}$, IJo, 1II)

from 14.20-16.20 h. 3/ $\mathrm{q}+\mathrm{Il}$ lost contact but joined subgroup again)

\section{April 25}

1. Guarea grandifolia

2. Tetragastris altissima

3. Tetragastris panamensis

4. Ficus trigonata

5. Clusia scrobiculata

6. Tetragastris panamensis

7. Guarea grandifolia

8. Tetragastris panamensis

9. Cecropia sciadophylla

10. Styrax aff. tanshawei

11. Tetragastris panamensis

12. Tetragastris altissima

13. Pachira insignis

12. Tetragastris altissima

I1. Tetragastris panamensis

14. Ficus pertusa

15. Heteropsis jenmani

16. Pachira insignis

17. Guarea grandifolia

18. Guarea grandifolia

19. Geissospermum sericeum

20. Guarea grandifolia

21. Inga alba

fruit
truit
fruit
figst
fruit
fruit
fruit
fruit
infructescence
fruit
fruit
fruit
y. seeds
fruit
fruit
figs
infructescence
flowers
fruit
fruit
fruit (juice)
fruit
bark

$\begin{array}{lr}\text { I } & 7 \\ 1 & 16 \\ 1 & 16 \\ 1 & 2 \\ \text { II } & 3 \\ \text { I } & 6 \\ \text { I } & 6 \\ 1 & 2 \\ \text { II } & 6 \\ \text { I } & 14 \\ \text { I } & 3 \\ \text { I } & 29 \\ \text { II } & 1 \\ \text { I } & 41 \\ \text { I } & 4 \\ \text { I } & -9 \\ \text { II } & 6 \\ \text { II } & 2 \\ \text { I } & 12 \\ \text { I } & 12 \\ \text { I } & 15 \\ \text { I } & 5 \\ \text { III } & 1\end{array}$


Table VIII (continued)

\begin{tabular}{|c|c|c|c|}
\hline Species & Food item & $\begin{array}{l}\text { Food } \\
\text { category }\end{array}$ & $\begin{array}{l}\text { Feeding } \\
\text { Time (min: }\end{array}$ \\
\hline
\end{tabular}

\section{April25}

22. Inga bourgoni

23. Moutabea guianensis

24. Spondias mombin

19. Geissospermun sericeum

17. Guarea grandifolia

20. Guarea grandifolia

25. Guarea grandifolia

26. Guarea grandifolia

27. Guarea grandifolia

12. Tetragastris aitissima

28. Guarea grandifolia

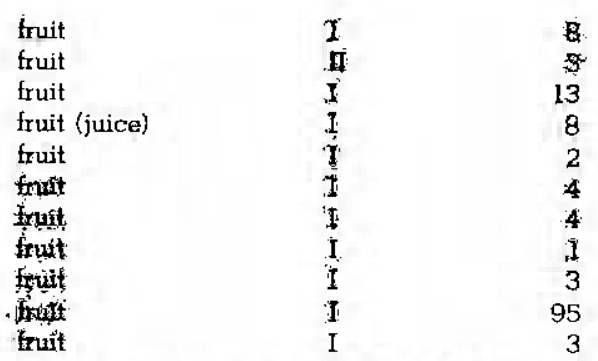

Weather: sunny. heavy rain from 13.54-14.04, 14.20-14.35 and 18.05-18.25 h.

Observation time: $5.35-17.35 \mathrm{~h}$

Day range size: $2,850 \mathrm{~m}$

Total food species used: 15

Total food items used: 16

Total feeding time: 365 minutes (343 on primary, 21 on secondary and 1 on tertiary food sources)

Most important food species calculated in feeding time: Tetragastris altissima (181 min.).

Guarea grandifolia (59 min.), Tetragastris panamensis (34 min.), Geissospermum sericeum (23 $\min$.

Subgroup size and composition: $5.35-8.00 \mathrm{~h}-5$ (20\%, 10, $1 \mathrm{J3}$ )

$8.00-17.30 \mathrm{~h}-7\left(1_{q}+1 \mathrm{j} 2 q\right.$ joined subgroup)

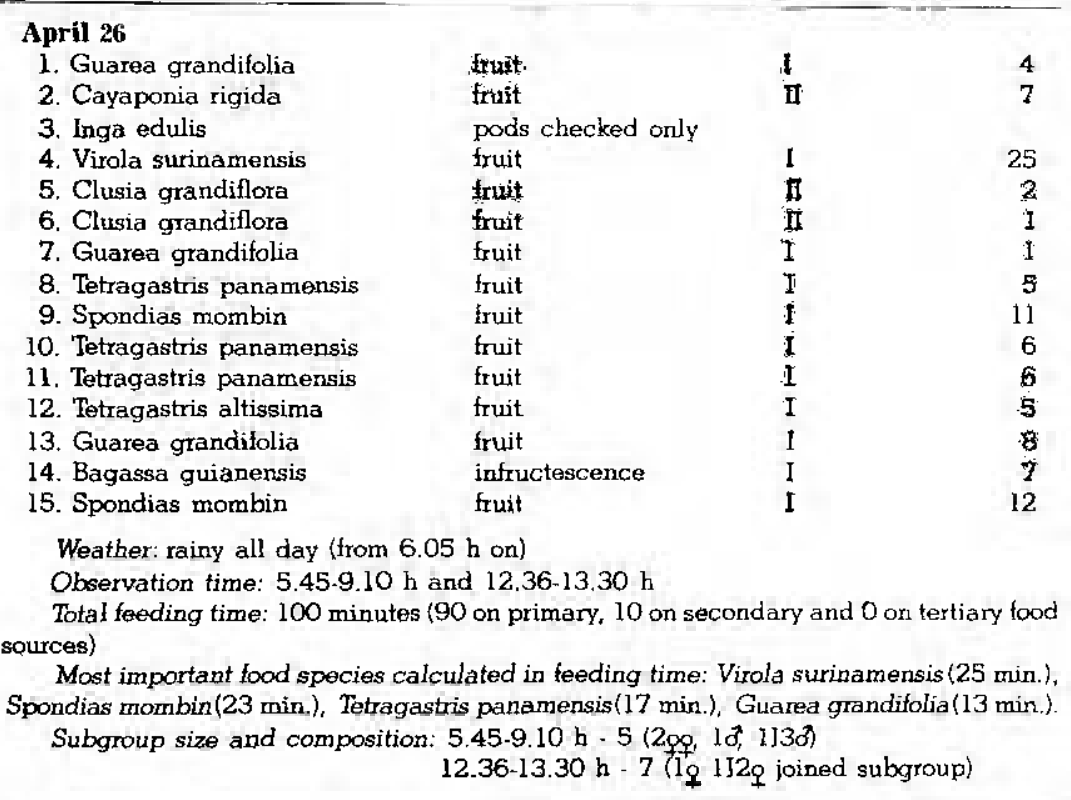




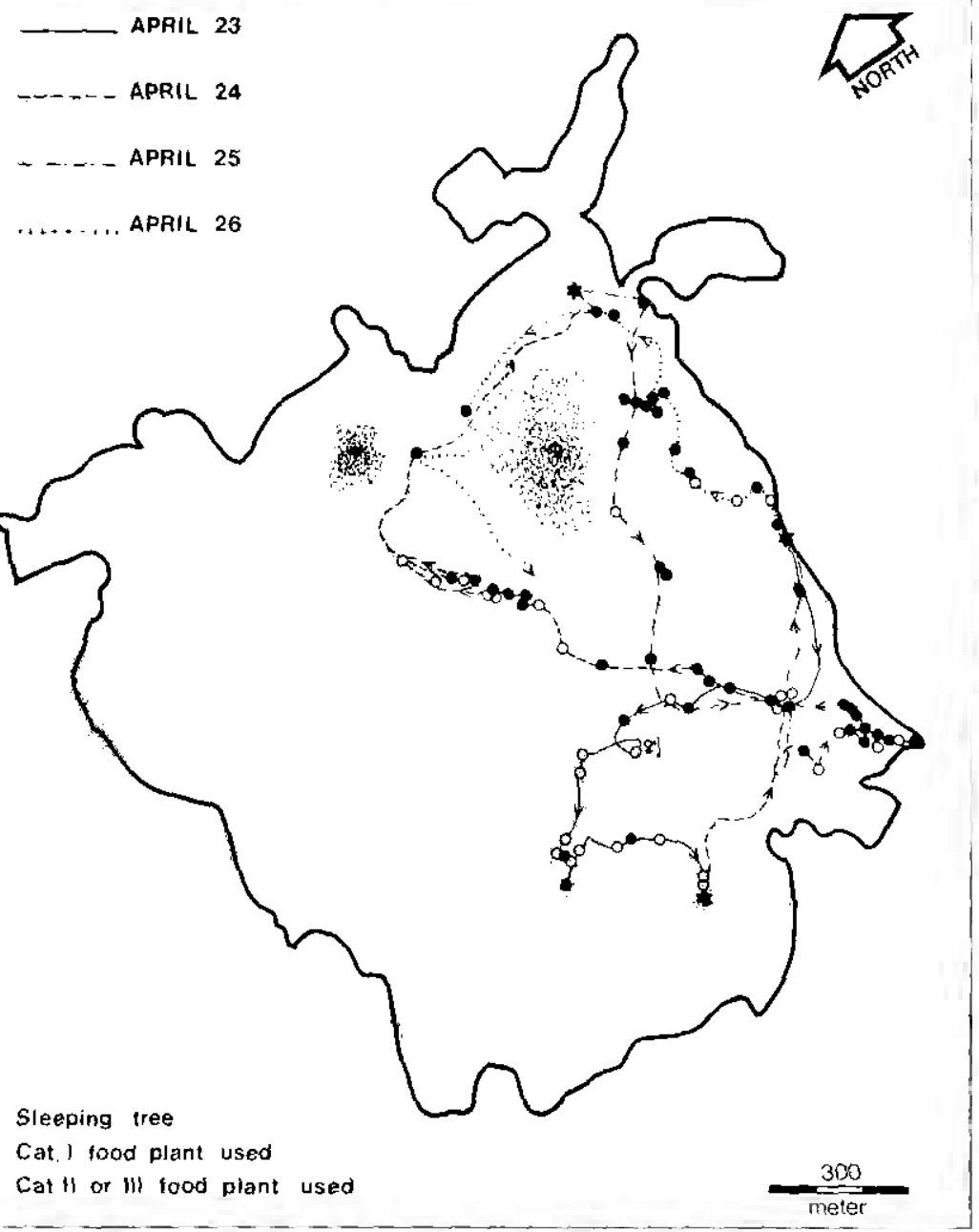

Figure 21 Routes followed by a spider monkey subgroup led by a certain female on fous consecutive days in April 1978. The tood sources used are indicated and correspond with those given in table VII. In this figure, I only indicate whether a food source belongs to category I or to category II or III. 

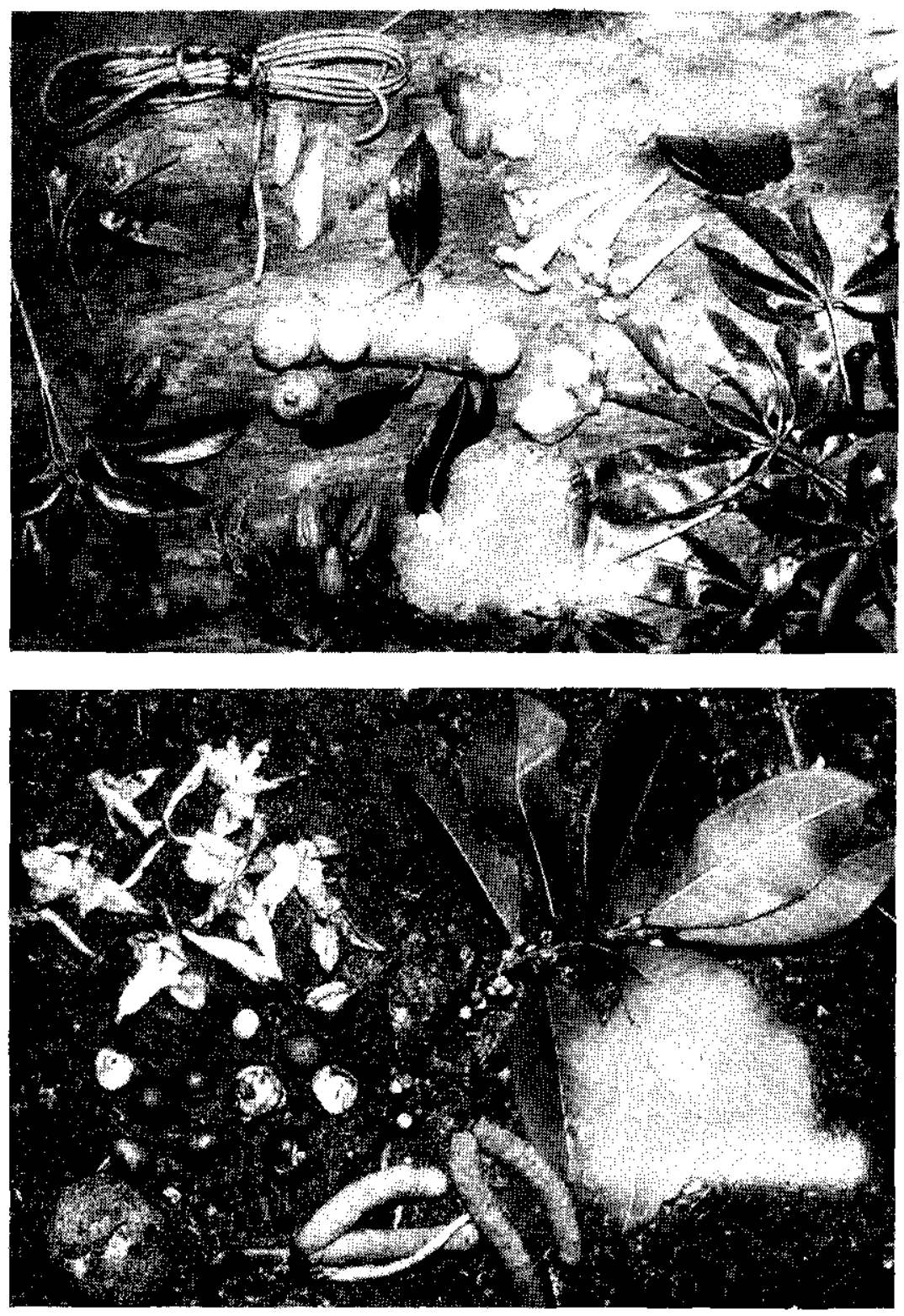

Figure 22 Food choice by a spider monkey subgroup on December $2(\mathrm{~A})$ and December 3,1977 (B). The food species are listed in table VII. Note the great number of tertiary food sources (category III) in day-sample $A$, and the lack of nutritious, large-seeded fruits at this time of year. 

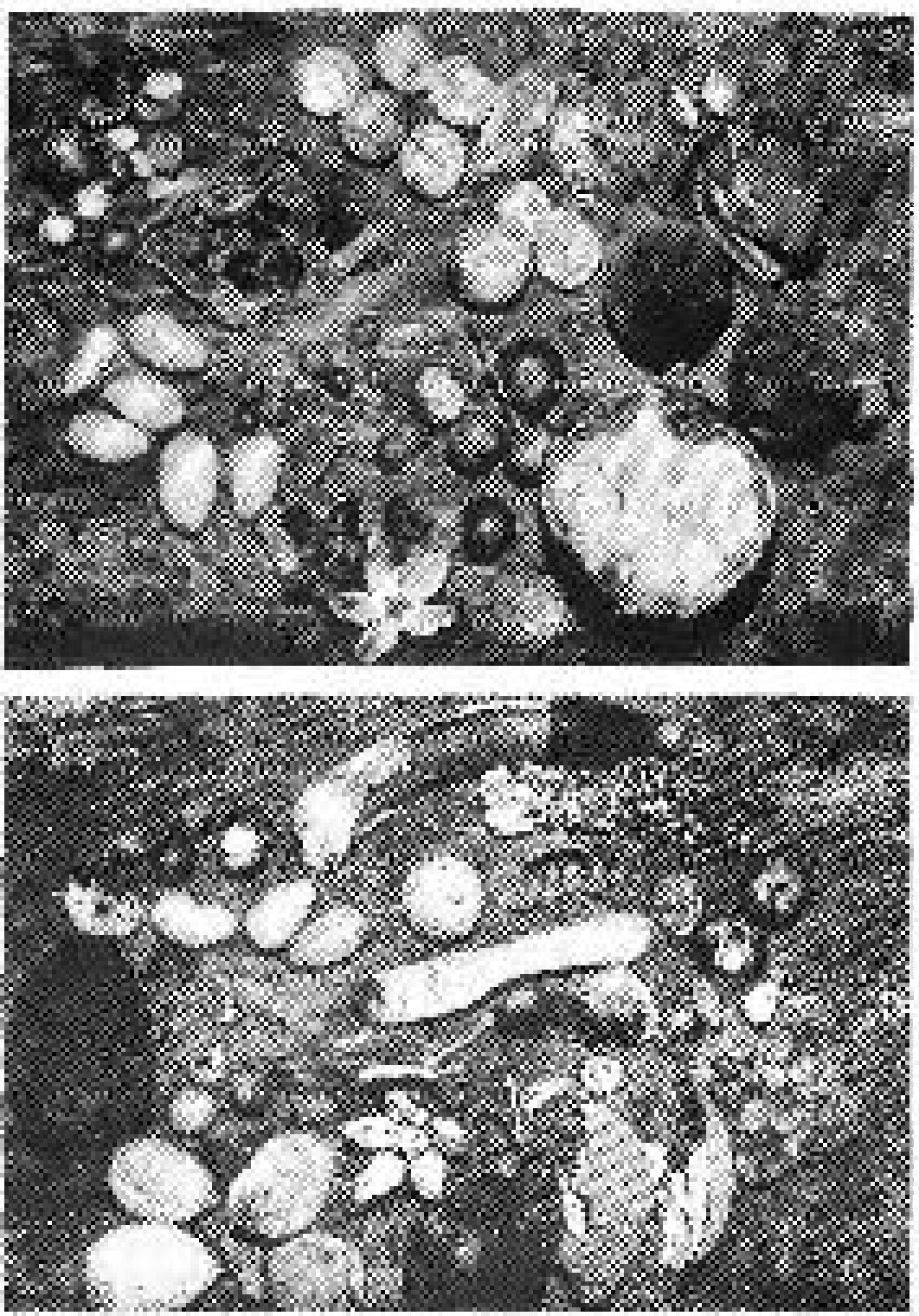

Figure 23 Food choice of a spider monkey subgroup on April 24 (A) and April 25, 1978 (B). The Food species are listed in table VIII. Note the great number of primary food sources (category I) and the lack of tertiary food sources (category III) in both day-samples. The truits are mainly of the nutritious, large-seeded type at this time of year. 
4.2.9. Monthly variation in food choice. In figure 24 , monthly variation in food choice of Ateles p. paniscus is shown graphically. It should be emphasized that total observation time for each month of the year was kept roughly equal. The striking variation in samples per month is due to the fact that activity patterns of the spider monkeys varied greatly, and this is positively correlated with fruit supply and negatively with the amount of leaves and flowers eaten. In August, for example, a total of only 45 feeding records were collected. This was related to long resting periods and few feeding bouts concentrating on a small number of food plants. A total of only 24 food species was used during August, whereas in the period September-June between 41 and 61 food species were used each month.

Fruits account for a relatively low percentage (less than 60\%) of total feeding records for each month during the first part of the long dry season (July, August and September). This period is characterized by a striking decline in the availability of edible fruits. In contrast, during the period January-July, truits account for a high percentage of total feeding records, with a maximum in May and June (over 95\%), and ripe fruits with a maximum in March and April (over $91 \%$ ). Young seeds were eaten in all months except for July and October, but they contribute very low percentages for most months (fig. 24). However, relatively high percentages of seed predation are found in May $(14.4 \%)$ and June $(8.8 \%)$, around the peak of the long wet season. By ingesting many protein and fat-rich young seeds during this period, the monkeys seem to stock up on energy for the coming months of food scarcity. Surinam Bushnegroes and Amerindians have noted this, and prefer to hunt spider monkeys during these months when the animals are said to be at their fattest.

Flowers are eaten throughout the year, but account for only a very low percentage $(0.5 .-3.0 \%)$ of total feeding records during the long wet season (March-July). However, flowers contribute a considerable part of total feeding records during the long dry season (July-December), with a peak in September $(28.1 \%)$ in the middle of the dry season. A minor peak is reached in February $(6.3 \%)$ during the short dry season.

Flush leaves are also eaten throughout the year, but account for only a very low percentage $(3.0 \%$ or less) of total feeding re. 


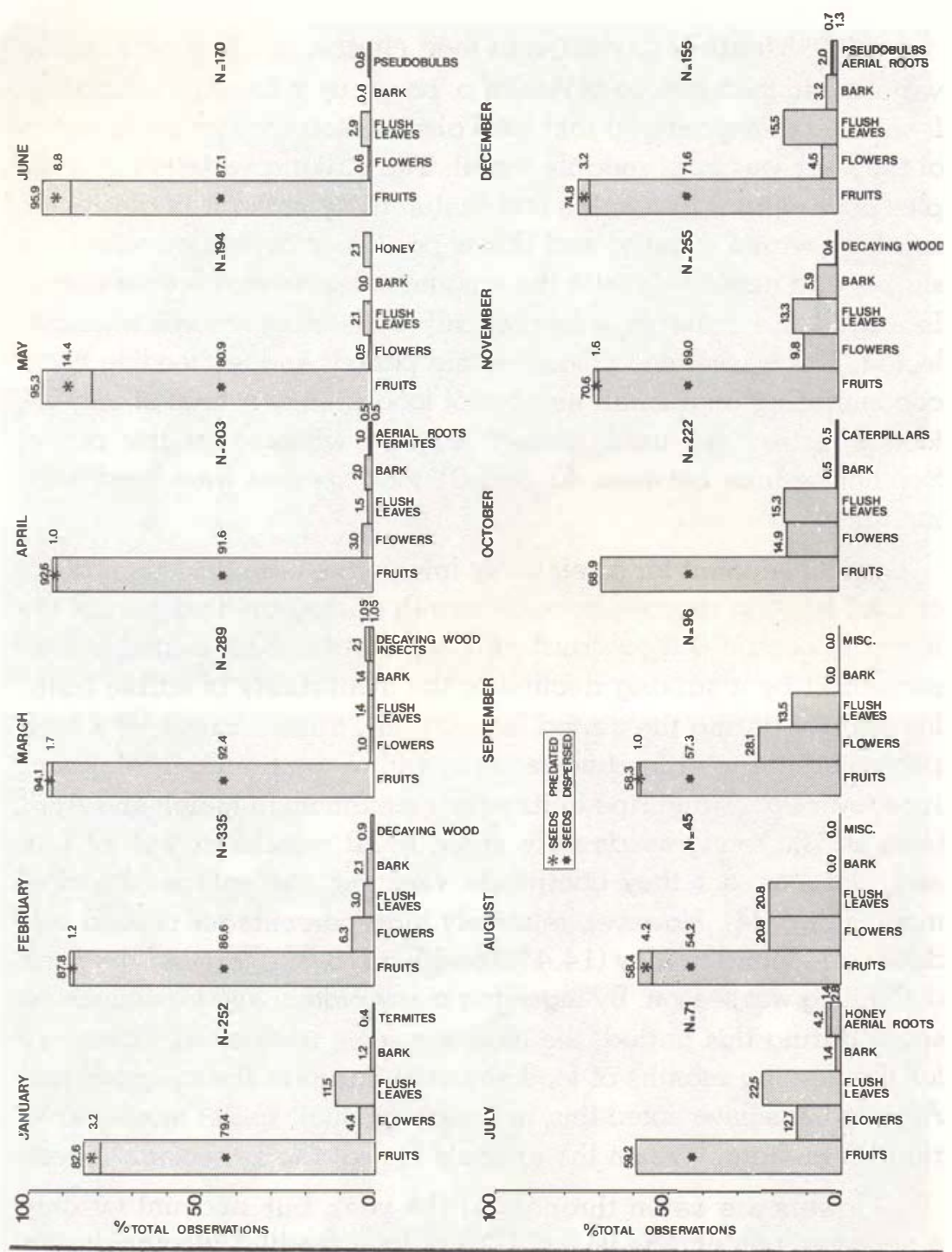

Figure 24 Monthly variation in food choice by a spider monkey group in the Voltzberg study area, expressed as percentage of the total number of feeding observations for each category.

cords during the months February-July. Flush leaves contribute relatively high percentages of total feeding records during the period July-February, that is from the start of the long dry season through the short wet season. 
Spidier monkeys rarely fed on bark $(0.0-2.1 \%)$. However, in November, feeding on bark accounted for $5.9 \%$, and in December for $3.2 \%$ of total feeding records. With the exception of July, bark-eating was not observed during the period May-October. Decaying wood and rotten palm sheaths were eaten only in February, March and November, but at very low trequencies. In February and March, these account for about $1 \%$, and in November for only $0.4 \%$ of total feeding records.

Aerial roots were eaten only in April, July and December, accounting for $0.5,2.8$ and $1.3 \%$ respectively of the total number of feeding records for each month.

Pseudobulbs were eaten only during June and December, accounting for 0.6 and $0.7 \%$ of total feeding records.

Honey was eaten only in May and July, accounting for 2.1 and $1.4 \%$ of total feeding records.

Insects appeared to be eaten only during part of the year, particularly in March. Termites were eaten during January, March and April, and accounted for $0.4,0.35$ and $0.5 \%$ respectively of total feeding records. Caterpillars were eaten only in March and October, accounting for 0.7 and $0.5 \%$.

During the months July, August and September (the period of low fruit supply), the monkey compensates its diet with relatively high percentages of both flowers and flush leaves (fig. 25). When fruit abundance is highest (during the period March.July), both flowers and flush leaves play a minor role in the spider monkey's diet.

The monthly diet of Ateles p. paniscus in the Voltzberg study area is given in detail and illustrated in Figures 26-37. All food items used are depicted and ranked according to the total number of feeding records for each item. The plant part actually eaten is also mentioned. Seeds and stones are usually drawn as well. The drawings show monthly diversity of food choice, and the relative importance for each food item in a particular month is given by the number of feeding records and its location in the drawing: the more centrally located, the greater the importance. 

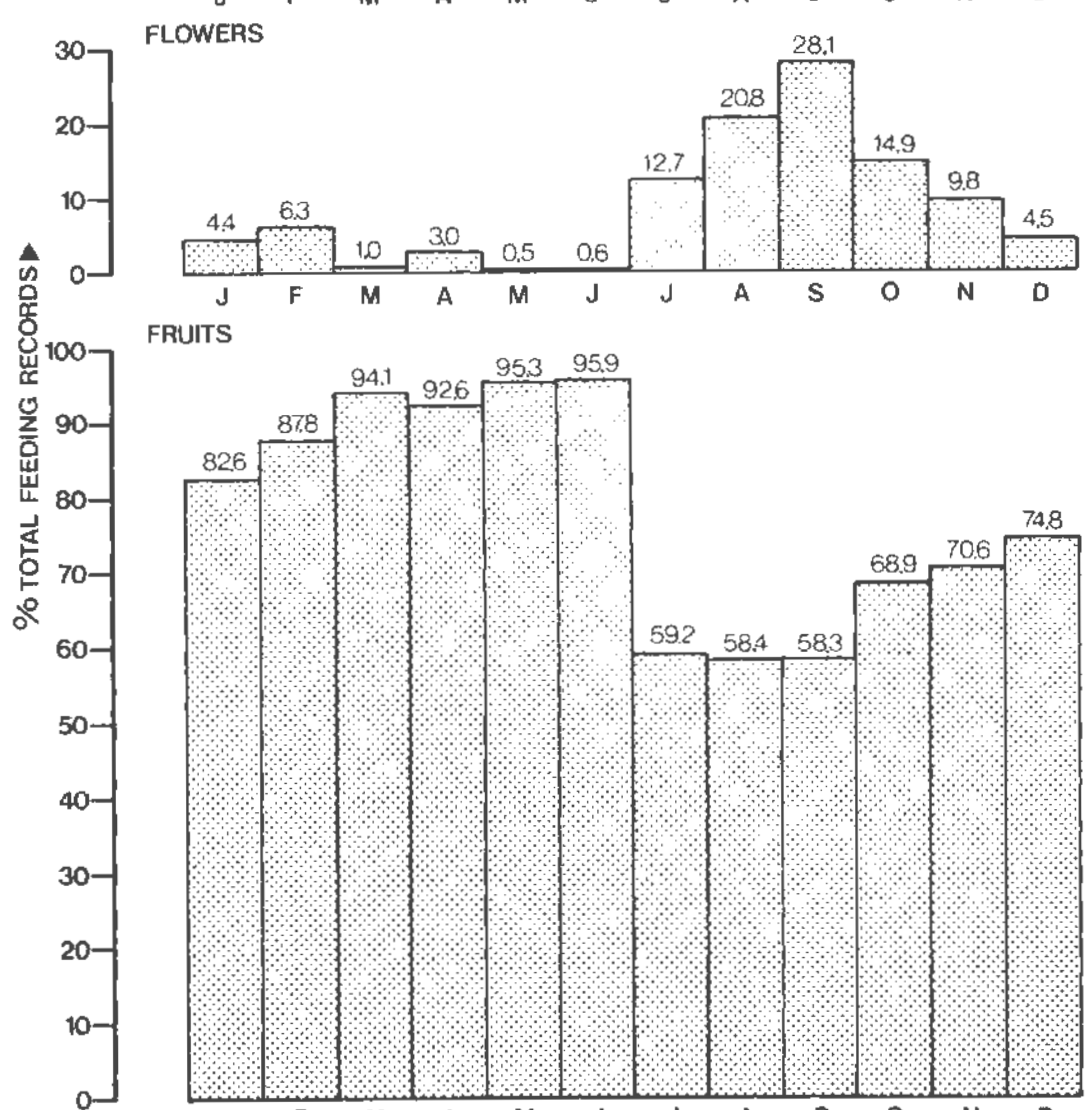

FRUITS

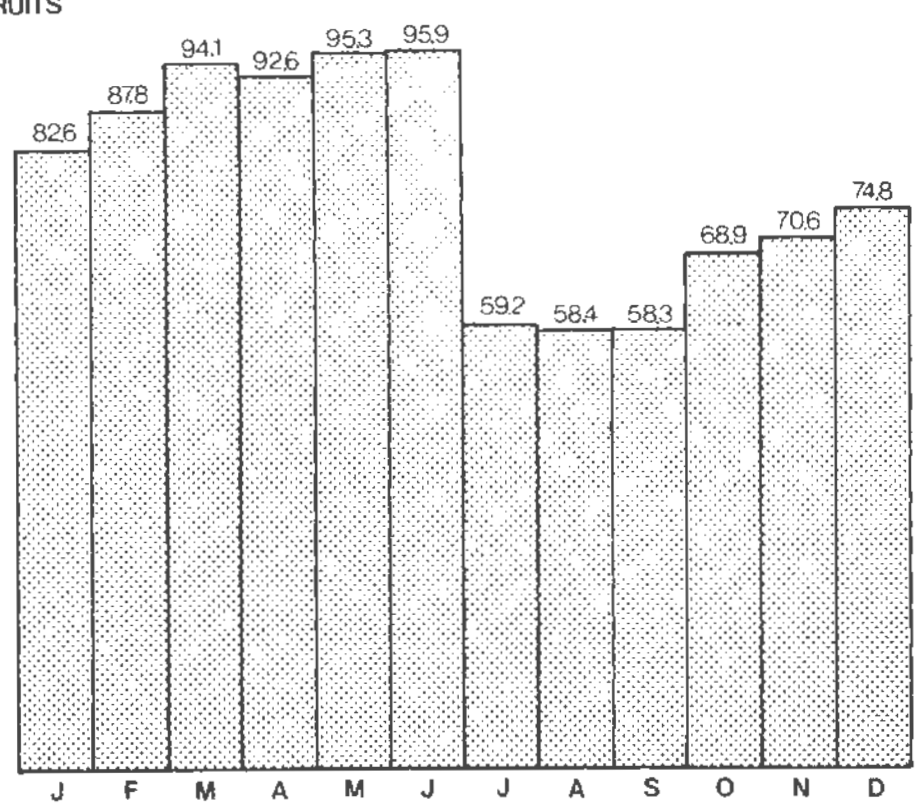

Figure 25 Comparison of monthly percentages of the total number of feeding records for flush leaves, flowers and fruits eaten by Ateles p. paniscus in the Voltzberg study area during the present study. 


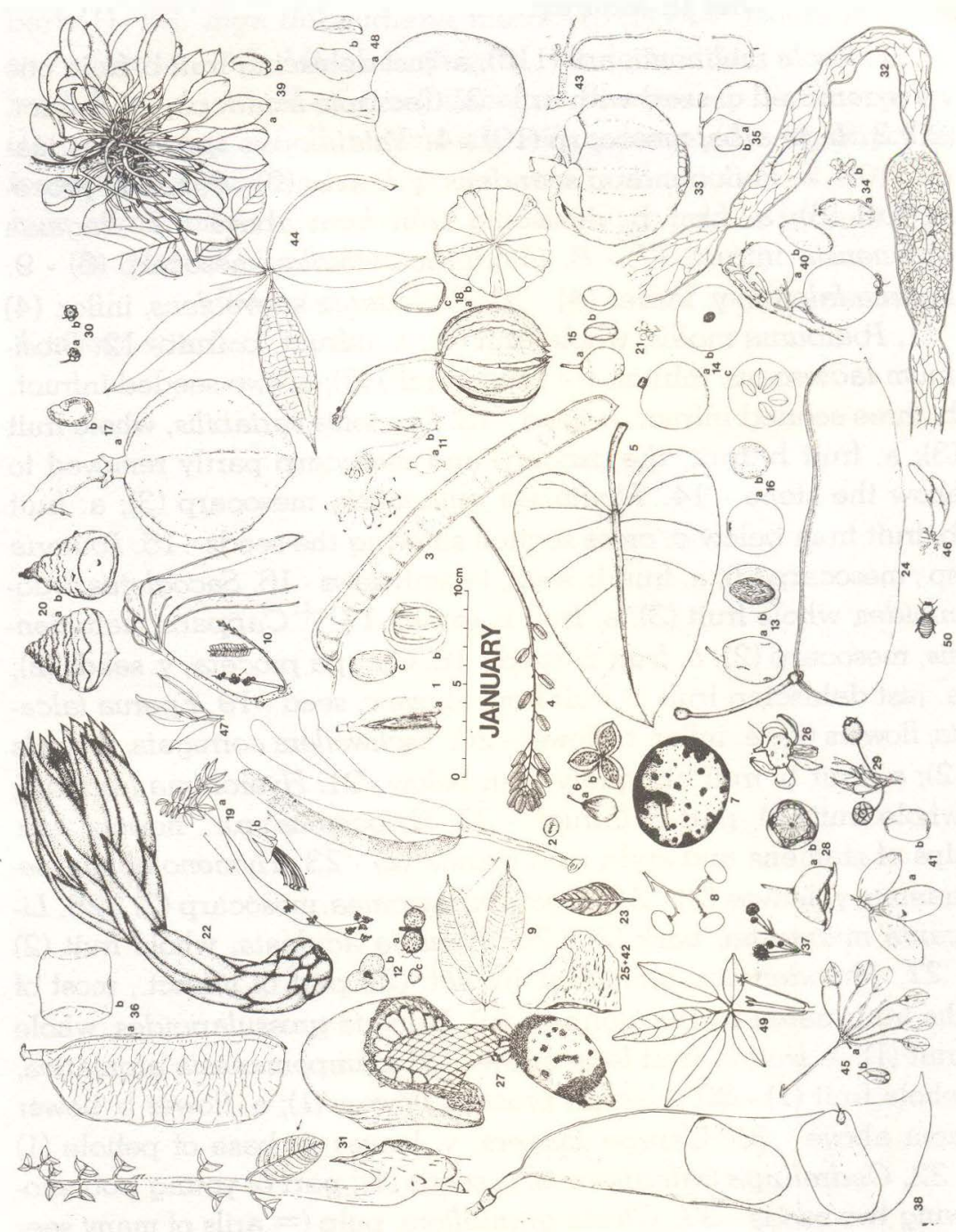

Figures 26-37 Monthly diet of Ateles p. paniscus in the Voltzberg study area. The species of food plant ano other food items are ranked according to the total number of feeding records collected for each month of the year (in parentheses). In addition, the part actually eaten is mentioned. When necessary, an explanation of the corresponding drawing of a food item is added. The drawings show monthly diversity of food choice, whereas the relative importance of a food item in a particular month is given by its location in the drawing. The more centrally located, the greater the importance.

$+=$ fruiting less than once every year; $y .=$ young; inflor. $=$ inflorescence $;$ infruct. $=$ infructescence 
Fig. 26. Diet in January.

1. Virola melinonii, aril (110); a. just dehiscing fruit b. fruit, one valve removed c. seed with aril - 2. Cecropia sciadophylla, infruct. (20) 3. Inga alba, mesocarp (10) - 4. Vataireopsis speciosa, y. leaves (10) - 5. Philodendron scandens, y. leaves (9) - 6. Laetia procera, aril (8); $a$. fruit $b$. dehiscing fruit from above - 7. Bagassa guianensis, infruct. (7) - 8. Cordia lomatoloba, mesocarp (6) - 9 . Eperua falcata, y. leaves (4) - 10. Piptadenia suaveolens, inflor. (4) - 11. Pourouma mollis, whole fruit (4); a. infruct. b. fruit - 12. Brosimum lactescens, infruct. (= pseudofruit) (3); a. two-seeded infruct. b. three-seeded infruct. c. seed - 13. Drypetes variabilis, whole fruit (3); a. fruit b. fruit, the exocarp and mesocarp partly removed to show the stone - 14. Moutabea guianensis, mesocarp (3); a. fruit b. fruit from below c. cross section showing the seeds -15 . Pouteria sp., mesocarp (3); a. fruit b. seed, lateral views - 16. Sacoglottis cydonioides, whole fruit (3); a. fruit b. stone - 17. + Capparis maroniensis, mesocarp (2); a. fruit b. seed - 18. Carapa procera, y. seeds (2); a. just dehiscing fruit b. fruit from above c. seed - 19. Eperua falcata, flowers (2); a. inflor, b. flower - 20. Eschweilera corrugata, y. seeds (2); $a$. fruit b. fruit obliquely from below -21 . Hyeronima laxiflora, whole fruit (2); part of infruct. - 22. Hylocereus sp.a., flowers, esp. tips of stamens and style, and stigma (2) - 23. Hymenolobium petraeum, y. leaves (2) - 24. Inga cinnamomea, mesocarp (2) - 25. Licania micrantha, bark (2) - 26. Pereskia aculeata, whole fruit (2) - 27. Philodendron acutatum, infruct. (2); part of infruct., most of the fruits eaten by the monkey - 28. Bellucia grossularioides, whole fruit (1); a. fruit b. fruit from above - 29 Campomanesia aromatica, whole fruit (1) - 30. Carapa procera, flowers (1); a. flower b. flower from above - 31. Carapa procera, y. leaves + base of petiole (1) - 32. Cedrelinga cateniformis, y. seeds (1); part of young pod showing two seeds - 33. Clusia grandiflora, pulp (= arils of many seedlets) (1) - 34. Combretum rotundifolium, y. seeds (1); a. fruit b. fruit from above. 35. Copaifera guianensis, aril (1); a. fruit b. seed - 36 . Dioclea macrocarpa, y. seeds (1); a. pod b. seed - 37. Enterolobium schomburgkii, inflor. (1) - 38. Eperua rubiginosa, y. seeds, esp. plumula (1); pod, one valve removed, showing the seeds - 39. Ephedranthus guianensis, mesocarp (1); a. infruct. b. seed - 40. Eugenia macrocalyx, whole fruit (1); a. infruct. b. seed - 41. Gnetum urens, perigonium or whole fruit (1); a. infruct. b. seed - 42. Inga alba, 
bark (1) - 43. Inga thibaudiana, mesocarp (1) - 44. Jacaratia spino$s a$, y. leaves - 45. Malmea obovata, whole fruit (1); a. part of infruct. b. seed - 46. Norantea guianensis, flowers, esp. the honeycups (1); part of inflor. 47. Peperomia glabella, y. leaves (1) - 48. Strychnos tomentosa, mesocarp (1); a. truit b. seed, lateral views - 49. Tabebuia serratifolia, y. leaves (1) - 50. Termites, whole insects (1) In total: 50 food items

45 tood plant species 252 feeding records

$I$ insect species 


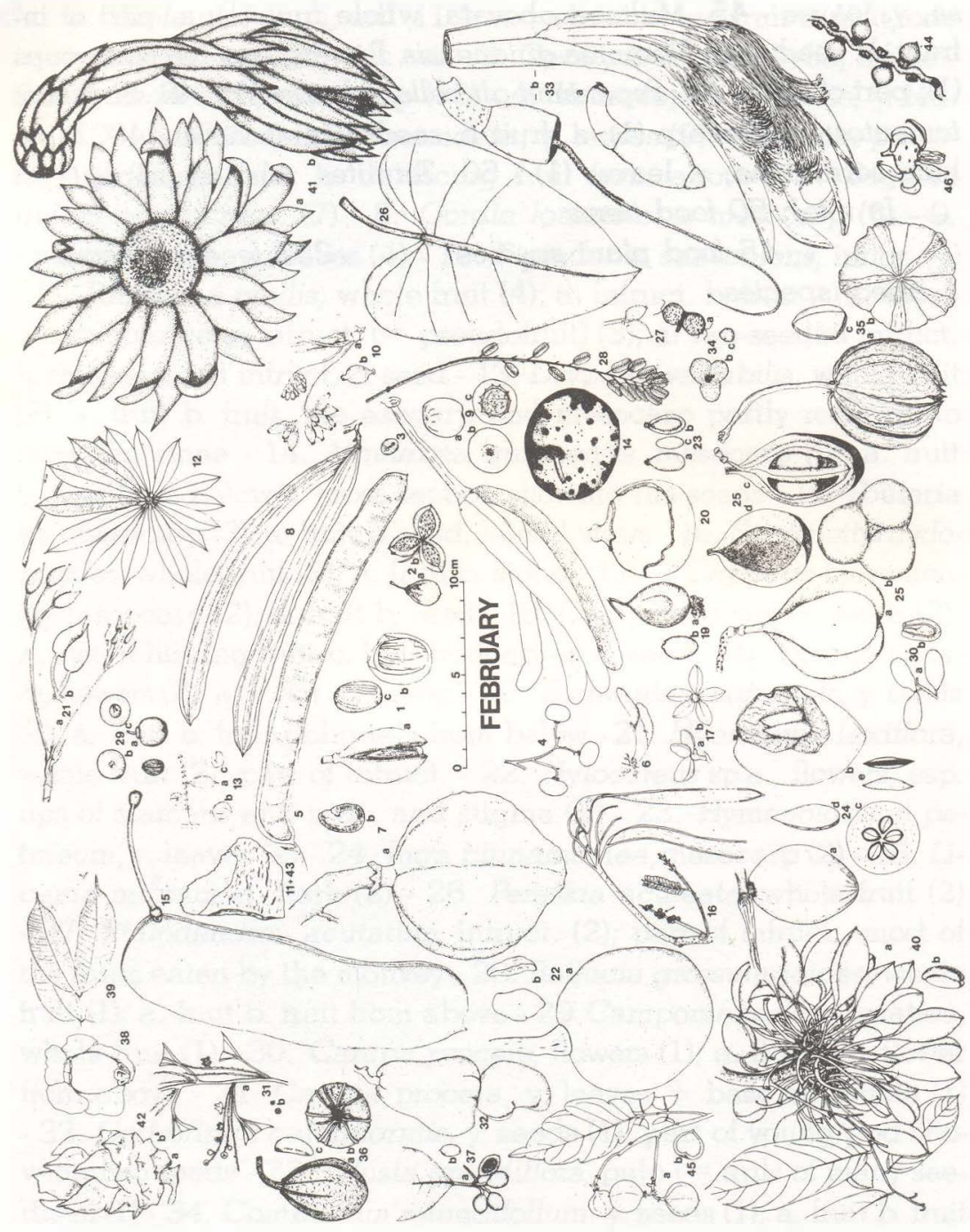

Fig. 27. Diet in February

1. Virola melinonii, aril (105); a just dehiscing fruit b. fruit, one valve removed c. seed with aril - 2. Laetia procera pulp (= arils of many seedlets) (32); a. fruit b. dehiscing fruit from above - 3. Cecropia sciadophylla, infruct. (25) - 4. Cordia lomatoloba, mesocarp (22) - 5. Inga alba, mesocarp (21) - 6. Norantea guianensis, flo- 
wers,esp. the honeycups (15); part of inflor. - 7. + Capparis maroniensis, mesocarp (12) a. truit b. seed - 8. Inga edulis, mesocarp (9) -9. Ampelocera edentula, mesocarp (8); a. fruit b. fruit from below c. cross section, showing one stone - 10. Pourouma mollis, whole fruit (7); a. infruct. b. fruit - 11. Licania micrantha, bark (6) - 12 . Cecropia sciadophylla, y. leaves (5) - 13. Paullinia acuminata, aril (5); a. infruct. b. other type of fruit c. seed with aril - 14. Bagassa guianensis, infruct. (4) - 15. Inga cinnamomea, mesocarp (4) - 16. Piptadenia suaveolens, inflor. (4) - 17. Quassia simarouba, mesocarp (4); a. fruit b. fruit from above - 18. Attalea Maripa, decaying and rotten sheaths of long before shed leaves (3) - 19. Cayaponia ophthalmica, mesocarp (3); a. fruit b. seed - 20. Licania majuscula, exocarp + mesocarp (3); fruit the outer layer partly scraped off by the monkey, showing the big stone - 21. Pourouma minor, whole fruit(3); a. young infruct. b. mature truits - 22. Strychnos tomentosa, mesocarp (3); a. fruit b. seed, lateral views - 23. Prieurella quadrijuga, aril (3); a. fruit b. longitudinal section c. seed - 24. Ecclinusa sp., y. seeds and mesocarp (2); $a$. fruit b. fruit partly opened by the monkey, showing some young seeds c. cross section d., e. seed, lateral views - 25. Paullinia spicata, aril (2); a. fruit b. fruit from above c. fruit, one valve removed d valve from inside -26 . Philodendron scandens. y. leaves (2) - 27. Pourouma sp., whole fruit (2); part of infruct. - 28. Vataireopsis speciosa, y. leaves (2) - 29. Vitex stahelii, whole fruit or mesocarp only (2); $a$. fruit $b$. fruit from above $c$. fruit from below - 30. Abuta grandifolia, mesocarp (1); a. part of infruct. with two types of truit $b$. stone - 31. Alchorneopsis floribunda, whole fruit (1); a. infruct. b. seed - 32. Anacardium giganteum, pseudofruit (= pedicel) (1) - 33 Pachira insignis, flowers + floral buds (I); a. floral bud b. flower - 34. Brosimum lactescens, intruct. (1); a. two-seeded b. three-seeded infruct. c. seed - 35. Carapa proce$r a$, y. seeds ( 1 ; $a$. just dehiscing truit $b$. fruit from above $c$. seed - 36. Cheiloclinium hippocrateoides, mesocarp (1); $a$. fruit b. fruit from below c. seed - 37. Chrysophyllum auratum, mesocarp (1); a. infruct b. seed - 38. Clusia scrobiculata, pulp (= arils of many seedlets) (1) - 39. Eperua falcata, y. leaves (1) - 40. Ephedranthus guianensis, mesocarp (1); a. infruct. b. seed - 41. Hylocereus sp. a., flowers, esp. tips of stamens and style, and stigma (1); a. flower from above b. flower, lateral view - 42. Hylocereus sp. b., whole fruit (1); a. fruit b. seed - 43. Inga alba, bark (1) - 44. Oenocarpus bacaba, 
whole fruit (1); part of intruct. - 45. Paullinia sphaerocarpa,y. seeds + aril $(1)$; a. infruct. b. valve trom inside - 46. Pereskia aculeata, whole fruit (1)

In total: 46 tood items 335 teeding records

44 food plant species 


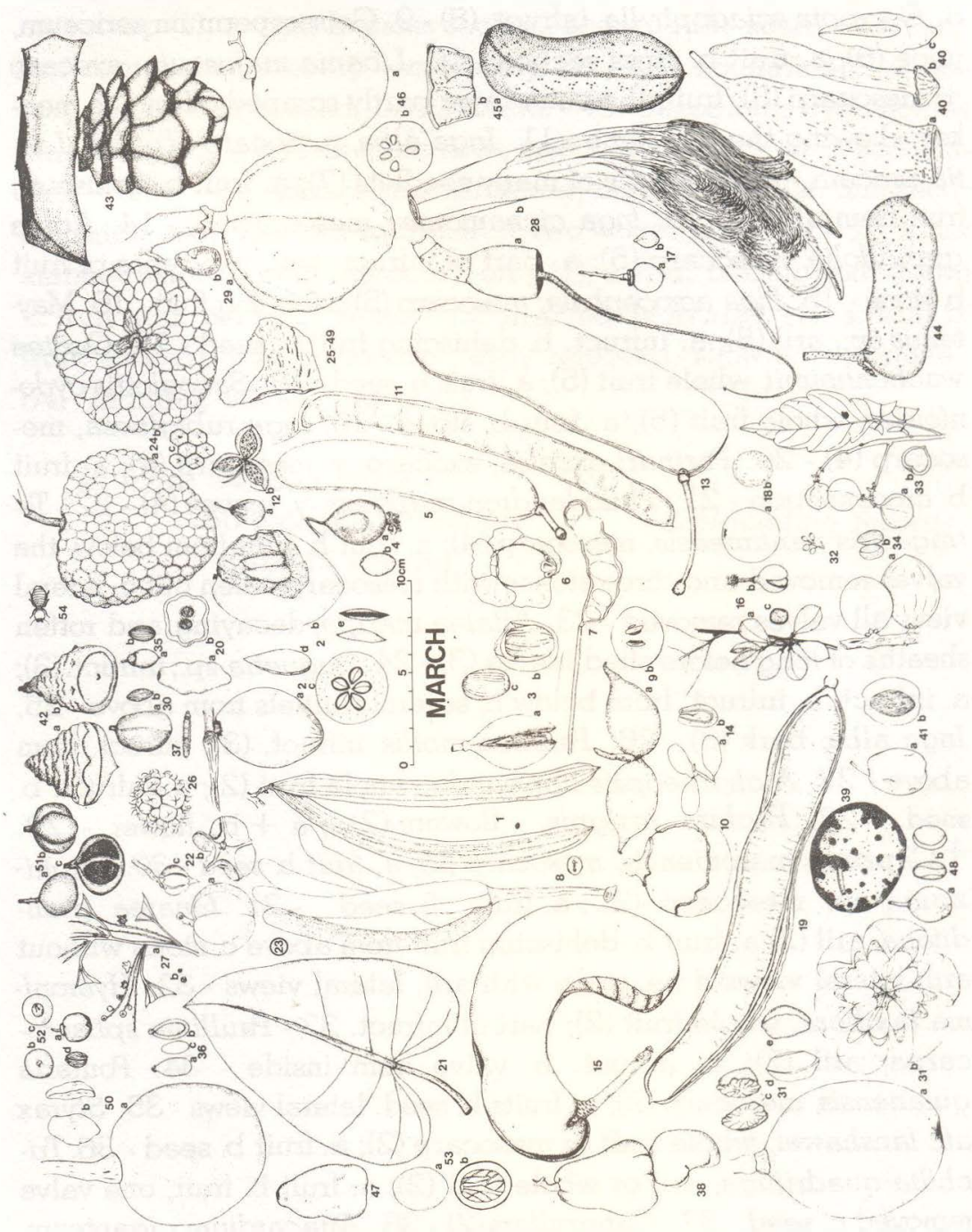

Fig. 28. Diet in March

1. Inga edulis, mesocarp (44) - 2. Prieurella sp.,mesocarp (38); a. fruit $b$. truit partly opened by the monkey, showing some seeds c. cross section d., e seed, lateral views - 3. Virola melinonii, aril (24); a. just dehiscing fruit b. fruit, one valve removed c. seed with aril - 4. Cayaponia ophthalmica, mesocarp (14); a. fruit b. seed 5. Inga bourgoni, mesocarp (13) - 6. Clusia scrobiculata, pulp (= 
arils of many seedlets) (10) - 7. Inga leiocalycina, mesocarp (9) . 8. Cecropia sciadophylla, infruct. (8) - 9. Geissospermum sericeum, juice (8); a. fruit b. cross section - 10. Licania majuscula, exocarp + mesocarp (8); fruit, the outer layer partly scraped off by the monkey, showing the big stone - 11. Inga alba, mesocarp (7) - 12. Laetia procera, pulp (= arils of many seedlets (7); a. fruit b. dehiscing fruit from above - 13. Inga cinnamomea, mesocarp (6) - 14. Abuta grandifolia, mesocarp (5); a. part of infruct. with two types of fruit b. stone - 15. Inga acrocephala; mesocarp (5) dehiscing fruit - 16. Maytenus sp., aril (5); a. infruct. b. dehiscing fruit c. seed - 17. Ocotea wachenheimii, whole fruit (5); a. fruit b. seed - 18. Sacoglottis cydonioides, whole fruit (5); a. fruit b. stone - 19. Inga rubiginosa, mesocarp (4) - 20. Parinari excelsa, exocarp + mesocarp (4); a. fruit b. cross section - 21. Philodendron scandens, y. leaves (4) - 22. Tetragastris panamensis, mesocarp (4); $a$. fruit $b$. fruit from below, the valves removed and three stones with mesocarp eaten out $c$. lateral view, all valves removed - 23. Attalea maripa, decaying and rotten sheaths of long before shed leaves (3) - 24. Duguetia sp., infruct. (3); a. infruct. b. infruct. from below c. several fruitlets from above -25 . Inga alba, bark (3) - 26. Perebea mollis, infruct. (3); infruct. from above - 27. Alchorneopsis floribunda, whole fruit (2); a. infruct, b. seed - 28. Pachira insignis, flowers (2); $a+b$. flower - 29. + Capparis maroniensis, mesocarp (2); a. truit b. seed - 30. Amphizoma sp. mesocarp (2); a. truit b. seed . 31. Guarea grandifolia, aril (2) a. fruit b. dehiscing fruit from above $c$. stone without aril, lateral views $\mathrm{d}+\mathrm{e}$. stone with aril, lateral views -32 . Hyeronima laxiflora, whole fruit (2); part of infruct. 33 - Paullinia sphaerocarpa, aril (2); a. infruct. b. valve from inside - 34. Pouteria guianensis, mesocarp (2); a. fruits b. seed, lateral views - 35. Styrax aff. fanshawei, whole fruit or mesocarp (2); a. fruit b. seed - 36. Trichilia quadrijuga, aril or whole fruit $(2)$; $a$. fruit $b$. fruit, one valve removed c. seed - 37. Caterpillars (2) * 38. Anacardium giganteum, pseudofruit (= pedicel) (1) - 39. Bagassa guianensis, infruct. (1) 40. Couratari guianensis, y. seeds $(1) ; a$. fruit without operculum b. columella with opercuium c. seed - 41. Drypetes variabilis, whole fruit (1); $a$. fruit b. fruit, the exocarp and mesocarp partly removed to show the stone - 42. Eschweilera corrugata, y. seeds (1); a. fruit b. fruit obliquely from below - 43. Hylocereus sp. a., whole fruit (1) - 44. Inga cf. capitata, mesocarp (1) - 45. Tanaecium noctur- 
num, y. seeds (1); a. fruit b. seed - 46. Parahancornia fasciculata mesocarp (1); a. fruit b. cross section, showing the seeds - 47. Parinari campestris, exocarp + mesocarp (1) - 48. Pouteria sp., mesocarp (1); a fruit b. seed, lateral views - 49. Sacoglottis cydonioides, bark (1) - 50. Strychnos tomentosa mesocarp (1); a. fruit b. seed, lateral views. - 51. Tetragastris altissima, mesocarp (1); a. one-seeded fruit $b$. more-seeded fruit $c$. fruit, two valves removed - 52 . Vitex stahelii, whole fruit or mesocarp only (1); $a$. fruit $b$. fruit from above c. fruit from below d. stone - 53. Iriartea exorrbiza (1); $a$. fruit b. fruit, exocarp and mesocarp partly removed, showing the stone54. Termites, whole insects (1).

In total: 54 food items

50 food plant species 289 feeding records

2 insect species 


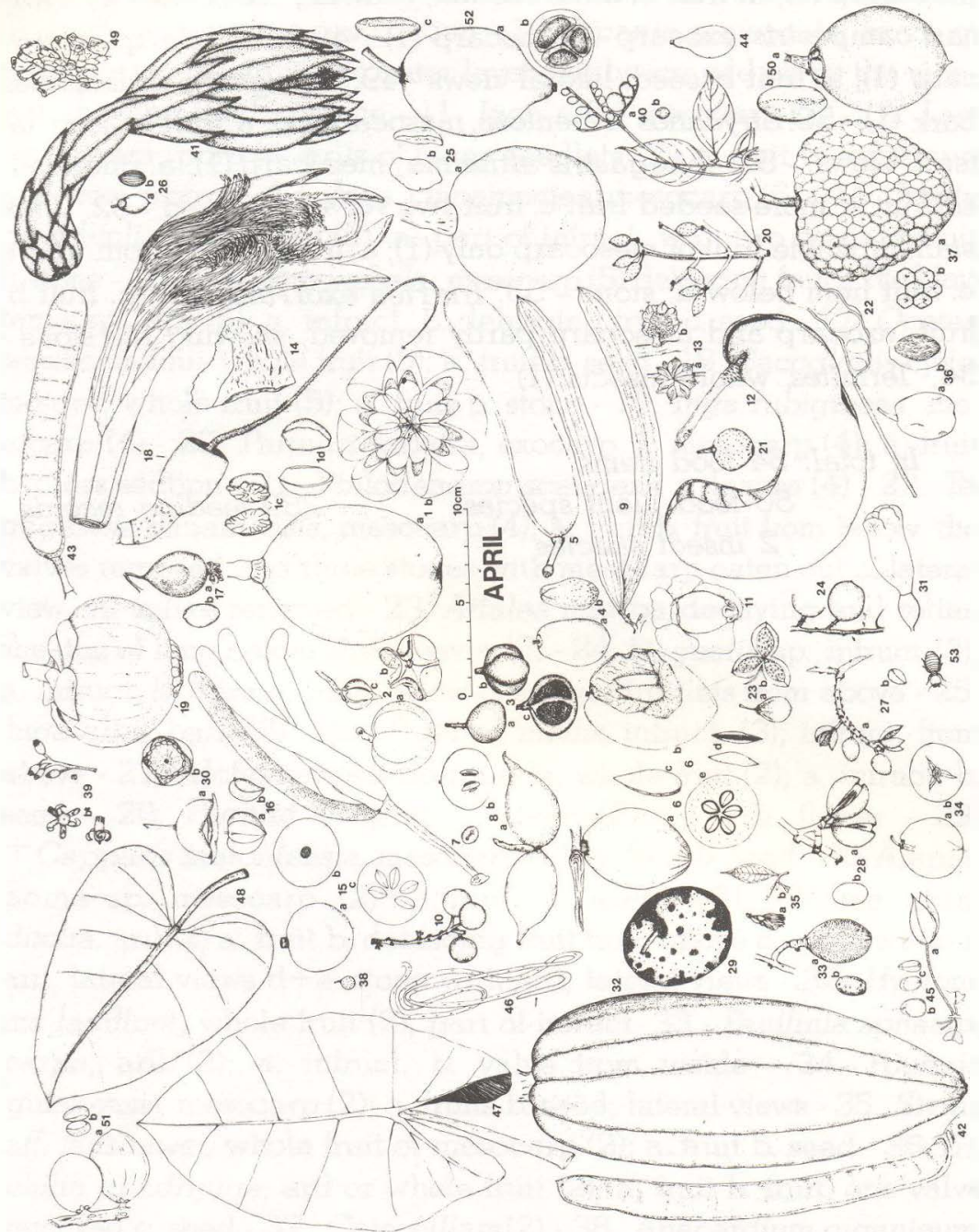

Fig. 29. Diet in April

1. Guarea grandifolia, aril (35); a. fruit b. dehiscing fruit from above $c$. stone without aril, lateral views $d$. stone with aril, lateral views - 2. Tetragastris panamensis, mesocarp (19); a. truit b. fruit from below, the valves removed and three stones with mesocarp eaten out c. lateral view, all valves removed - 3. Tetragastris altissi- 
ma, mesocarp (14); a. one-seeded fruit b. more-seeded truit c. fruit, two valves removed - 4. Spondias mombin, mesocarp (12); a. fruit b. stone - 5. Inga bourgoni, mesocarp (10) - 6. Prieurella sp., mesocarp (8); $a$. fruit $b$. fruit, partly opened by the monkey, showing some seeds $c+d$. seed, lateral views e. cross section - 7. Cecropia sciadophylla, infruct. (7) - 8. Geissospermum sericeum, juice (7); a. truit b. cross section - 9 . Inga edulis, mesocarp (7) - 10. Cayaponia rigida, whole fruit (6); infruct. - 11. Clusia scrobiculata, pulp (= arils of many seedlets) (6) - 12. Inga acrocephala, mesocarp (5) 13. Helicostylis tomentosa, infruct. (4); $a$. infruct from above b. infruct. obliquely from below - 14. Inga alba, bark (4) - 15. Moutabea guianensis, mesocarp (4); $a$. fruit $b$. fruit from below $c$. cross section showing the seeds - 16. Protium neglectum, mesocarp (4); a. more-seeded fruit b. one-seeded fruit - 17. Cayaponia ophthalmica, mesocarp (3); a. fruit b. seed - 18. Pachira insignis, flowers (2) - 19. Clusia grandiflora pulp (= arils of many seedlets) (2) - 20. Coussarea paniculata, whole fruit (2); intruct. - 21. Ficus trigonata, whole figs (2) - 22. Inga leiocalycina, mesocarp (2) - 23. Laetia procera, pulp (=arils of many seedlets) (2); a. truit b. dehiscing fruit from above - 24. Protium polybotryum, mesocarp (2); part of infruct. - 25. Strychnos tomentosa, mesocarp (2); a. fruit b. seed, lateral views - 26. Styrax aff. fanshawei, whole fruit or mesocarp only (2); a. fruit b. seed - 27. Trichilia martiana, aril or whole truit (2); a. infruct. b. seed - 28. Virola surinamensis, aril (2); a. part of infruct. with dehiscing fruits b. seed - 29. Bagassa guianensis, infruct. (1) - 30. Bellucia grossularioides, whole fruit (1); $a$. fruit b. fruit from above - 31. Species c. (Bignoniaceae), flowers (1) - 32. Pachira insignis, y. seeds ( 1 ); young truit - 33. Cheiloclinium cognatum, (young) seeds (1); a. truit b. seed - 34. Cheiloclinium anomalum, mesocarp (1); a fruit b. fruit from below - 35. Cynometra marginata, $y$. leaves (1); a. leaf bud b. y. leaf - 36. Drypetes variabilis, whole fruit (1); $a$, fruit $b$. fruit, exocarp and mesocarp partly removed to show the stone - 37. Duguetia sp., infruct. (1) a. infruct. b. several truitlets from above - 38. Ficus pertusa, whole figs (1) - 39. Guarea grandifolia, flowers (1); a. part of intlor. with floral buds b. flower - 40. Guettarda acreana, whole fruit (1); a. infruct. b. seed - 4l. Hylocereus sp. a., flowers, esp. tips of stamens and style, and stigma (1) - 42 . Inga alba, mesocarp (I) - 43. Inga stipularis, mesocarp (1) - 44. Licania densiflora, exocarp + mesocarp (1) - 45. Mendoncia hoff- 
mannseggiana, mesocarp (1); $a$, fruit $b$. fruit, the bracts removed c. stone - 46. Philodendron acutafum, tips and epiderm of aerial roots (1); part of aexial root - 47. Philodendron acutaturn, y. leaves (1) - 48. Philodendron scandens, y. leaves (1) - 49. Heteropsis jenmani, infruct. (1); part of infruct., several fruitlets eaten off - 50 . Platonia insignis, aril (1); a. fruit b. seed - 51. Pouteria guianensis, mesocarp (1); a. fruits b. seed, lateral views - 52. Rheedia macrophylla, aril $\{1\}$; a. truit b. cross section c. seed - 53. Termites, whole insects (1)

In total: 53 food items

48 tood plant species 203 feeding records

1 insect species 


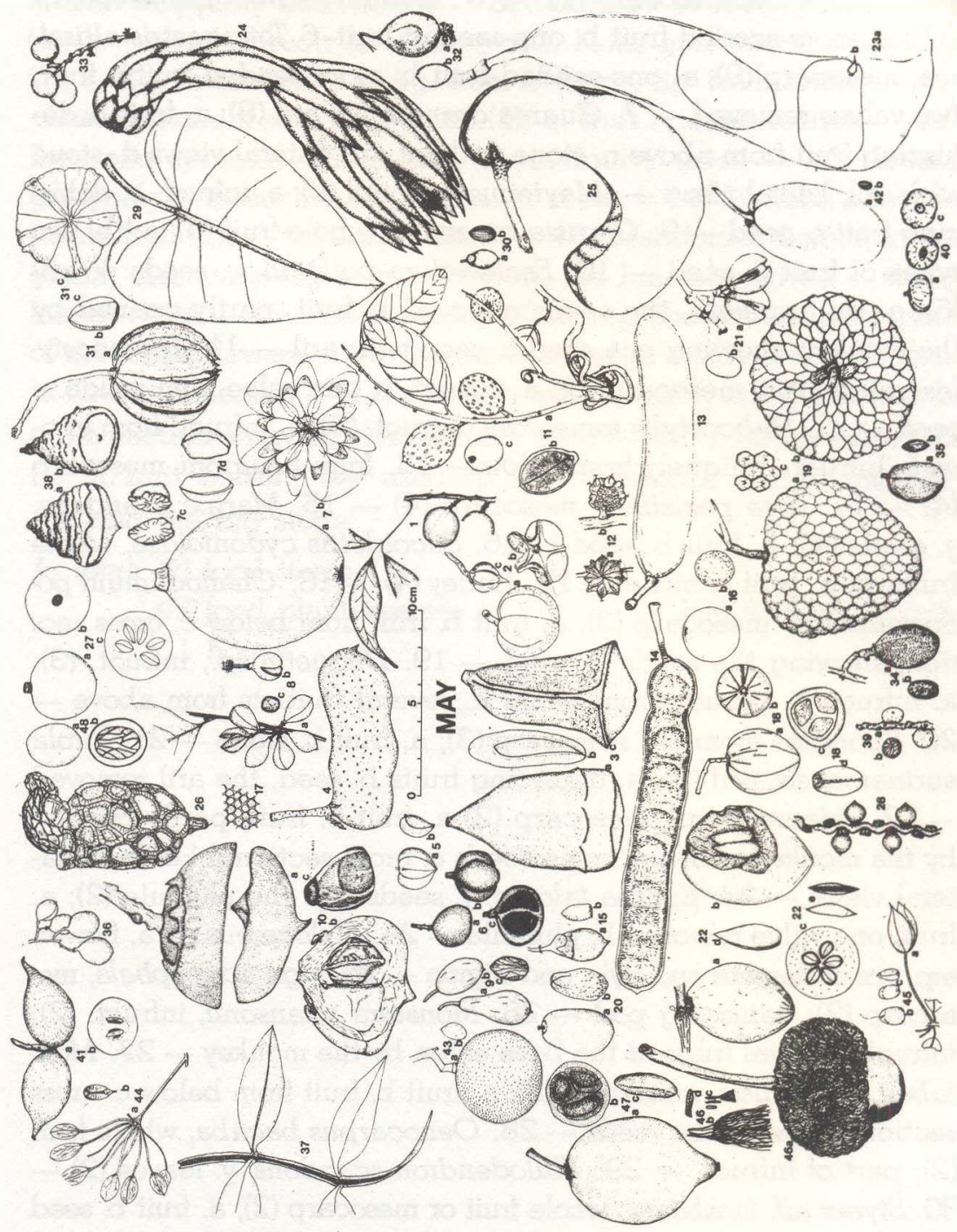

Fig. 30. Diet in May

1. protium polybotryum, mesocarp (24) -2 . Tetragastris panamensis, mesocarp (21); a. fruit b. fruit from below, the valves removed and three stones with mesocarp eaten out $c$. lateral view; all valves removed -3 Couratari stellata, young seeds + base of columella (13); a. fruit b. seed c. columella + operculum - 4. In- 
ga. cf. capitata, mesocarp (11) - 5. Protium neglectum, mesocarp (11); a. more-seeded truit b. one-seeded truit-6. Tetragastris altissima, mesocarp (9); a. one-seeded fruit b. more seeded fruit $c$. truit, two valves removed -7 . Guarea grandifolia, aril (8); a. fruit b. dehiscing fruit from above c. stone without aril, lateral views $d$. stone wich aril, lateral views - Maytenus sp., aril (8); a. infruct. b. dehiscing fruit c. seed -9 . Clarisia racemosa, whole fruit $(6)$; $a+b$. two types of fruit c. seed - 10. Eschweilera poiteaui, y. seeds + aril (6); a. fruit opened, the seeds removed b. fruit, partly opened by the monkey, showing one seed c. seed with aril - 11. Dicranostyles guianensis, mesocarp (5): $a$. infruct. $b$. one valve from inside $c$. seed - 12. Helicostylis tomentosa, infruct. (5); a. infruct. from above b. infruct. obliquely from below - 13. Inga bourgoni, mesocarp (4) - 14. Inga pezizifera, mesocarp (4) - 15. Maripa scandens, y. seeds (4); a. fruit b. seed -16 . Sacoglottis cydonioides, whole truit (4); a. fruit b. stone - 17. Honey (4) - 18. Cheiloclinium podostemmum, mesocarp (3); $a$. truit b. fruit from below c. cross section, showing the seeds d. seed - 19. Duguetia sp., intruct. (3); a. infruct. b. infruct. from below $c$. several fruitlets from above 20. Spondias mombin, mesocarp (3); a. fruit b. stone - 21. Virola surinamensis, aril (3); a. dehiscing fruits $b$. seed, the aril removed - 22. Prieurella sp., mesocarp (2) a. fruit $b$. fruit, partly opened by the monkey, showing some seeds $c$. cross section d+e. seed, lateral views - 23. Eperua falcata, y. seeds esp. the plumula (2); a. fruit, one valve removed b. plumula -24 . Hylocereus sp. a, flower, esp tips of stamens and style, and stigma - 25. Inga acrocephala, mesocarp (2); dehiscing pod - 26. Monstera adansonii, infruct. (2); infruct., several fruits at the base eaten by the monkey -- 27. Moutabea guianensis, mesocarp (2); $a$. fruit $b$. fruit from below $c$. cross section, showing the seeds - 28. Oenocarpus hacaba, whole fruit (2); part of infruct. -29 . Philodendron scandens, y. leaves (2) 30. Styrax aff. tanshawai, whole fruit or mesocarp (2); a. fruit b. seed - 31. Carapa procera, y. seeds (1); a. dehiscing truit b. y. seed c. truit from above - 32. Cayaponia ophthalmica, mesocarp (1); a. fruit b. seed - 33. Cayaponia rigida, whole fruit (1); infruct. -34 . Cheiloclinium cognatum, (young) seeds (1); a. truit b. seed - 35 . Amphizoma sp, mesocarp (1); a. fruit b. seed - 36. Dialium guianense, aril (1); part of infruct. - 37. Dipteryx odorata, y. leaves $(1)-38$. Eschweilera corrugata, y. seeds + aril (1); a. fruit 
b. fruit obliquely from below - 39. Euterpe oleracea, whole fruit (1); a. part of infruct. b. stone -40 . Ficus gomelleira, whole fig (1); a. fig, lateral view b. fig, from below c. fig from above -41 . Geissospermum sericeum, juice (1); $a$. fruit b. cross section -42 . Jacaratia spinosa, whole fruit (1); a. fruit b. seed - 43. Leonia glycycarpa, mesocarp (1); a. fruit b. seed - 44. Malmea obovata, whole fruit (1); a. infruct. b. seed - 45. Mendoncia hottmannseggiana, mesocarp (1); a. fruit b. fruit, the bracts removed c. stone, lateral views - 46. Parkia nitida, inflor, esp. the stamens and styles of the fertile flowers at the top ( 1$)$; $a$. inflor. b. sterile flowers c. fertile flowers $\mathrm{d}$. leaflet - 47. Rheedia macrophylla, aril (1); $\mathrm{a}$. fruit $\mathrm{b}$. cross section showing the seeds $\mathrm{c}$. seed - 48. Iriartea exorrhiza (1); a. fruit b. fruit, the exocarp and mesocarp partly removed, showing the big stone.

In total: 48 food items

47 food plant species $\quad 194$ feeding records

1 honey 


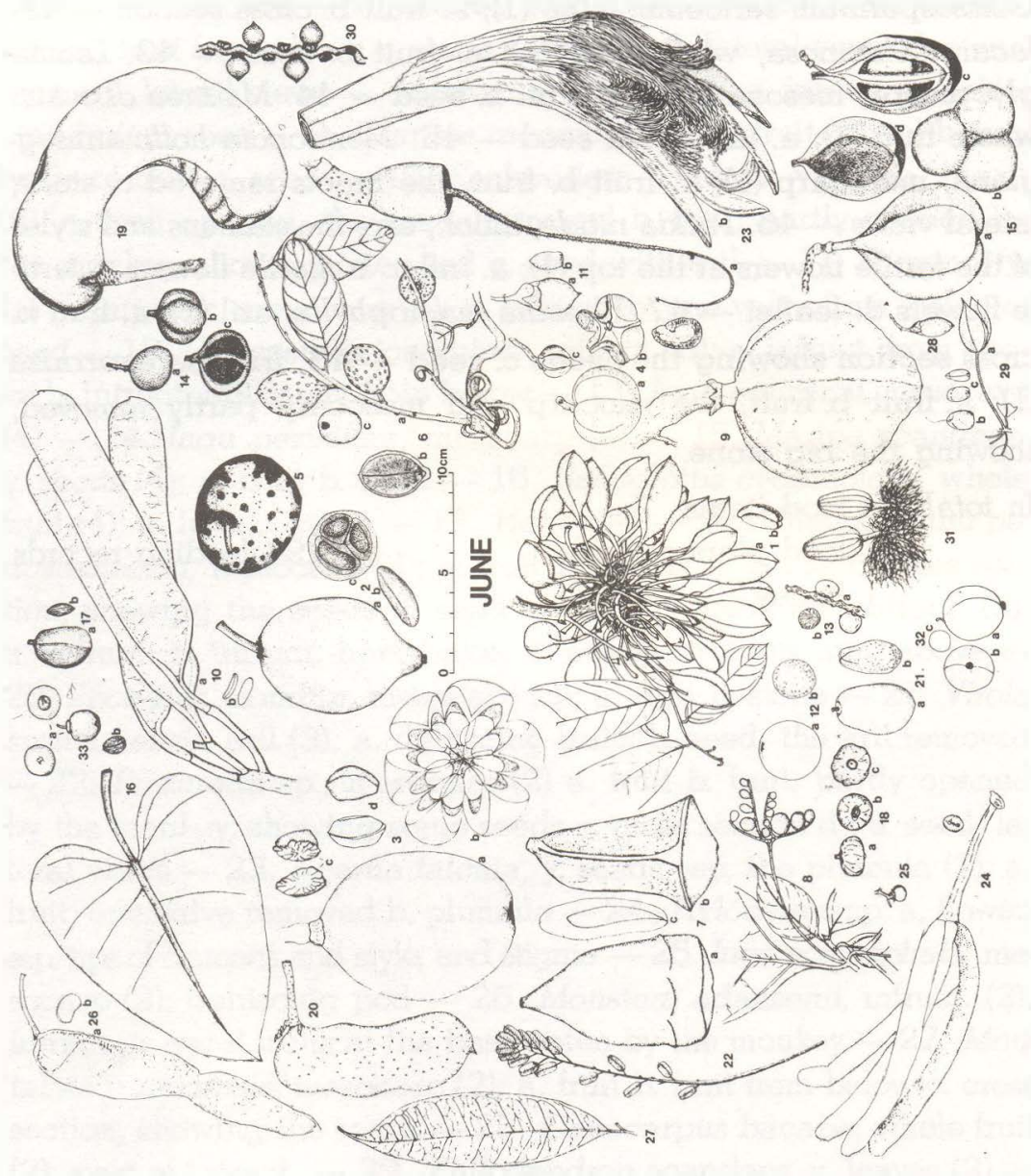

Fig. 31. Diet in June

1. Ephedranthus guianensis, mesocarp (29); a. intruct. b. seed - 2. Rheedia macrophylla, aril (20); a. fruit b. seed c. cross section, showing the seeds - 3. Guarea grandifolia, aril (17); a. fruit b. dehiscing fruit from above c. stone without aril, lateral views $d$. stone with aril, lateral views - 4. Tetragastris panamensis, mesocarp (11); a. fruit b. fruit from below, the valves removed and three 
stones with mesocarp eaten out c. lateral view, all valves removed -5 . Bagassa guianensis, infruct. $(10)-6$. Dicranostyles guianensis, mesocarp (10); a. infruct. b. valve from inside c. seed - 7. Couratari stellata, y. seeds + columella (9); a. fruit b. columella + openculum c. seed - 8 Guettarda acreana, whole fruit (7); intruct. - 9. Capparis maroniensis, mesocarp (6); a. fruit b. seed - 10. Dimorphandra pullei, desiccated mesocarp (5); a. part of infruct. b. seed, lateral views -- 11. Maripa scandens, y. seeds (5); a. fruit b. seed - 12. Sacoglottis cydonioides, whole truit (5); $a$. fruit $b$. stone - 13. Euterpe oleracea, whole fruit (4); a. part of intruct. b. stone - 14. Tetragastris altissima, mesocarp (4); a. one-seeded fruit b. more-seeded fruit, two valves removed - 15. Paullinia spicata, aril (3); $a$. fruit $b$. fruit from above $c$. fruit, one valve removed, showing the seeds $d$. valve from inside -16 . Philodendron scandens, y. leaves (3) - 17. Amphizoma sp., mesocarp (2); a. fruit b. seed - 18. Ficus gomelleira, whole fig (2); a. fig, lateral view b fig. from below c truit from above - 19. Inga acrocephala, mesocarp (2); dehiscing pod - 20. Inga cf. capitata, mesocarp (2)-21. Spondias mombin, mesocarp (2); a. fruit b. stone -22 . Vataireopsis speciosa, y. leaves (2) -23 . Pachira insignis, flowers, esp. the tips of stamens and style (1); $a+b$. flower -24 . Cecropia latiloba infruct. (1) - 25. Cordia panicularis, whole truit (1) - 26. Inga coriacea, mesocarp (1); a. fruit b. seed - 27. Maquira guianensis, y. leaves (1) - 28. Maripa glabra, y. seeds (1) - 29. Mendoncia hoffmanseggiana, mesocarp (1); $a$. fruit $b$. fruit, the bracts removed c. stone, lateral views - 30. Oenocarpus bacaba, whole fruit (1); part of intruct. 31. - Species a. (Orchidaceae), pseudobulbs (1) 32. Trymatococcus paraensis, whole fruit (1); $a$. fruit b. fruit from above c. seed - 33. Vitex stahelii, whole fruit or mesocarp (1); a. fruit, different views b. stone.

In total: 33 food items

170 feeding records

33 food plant species 


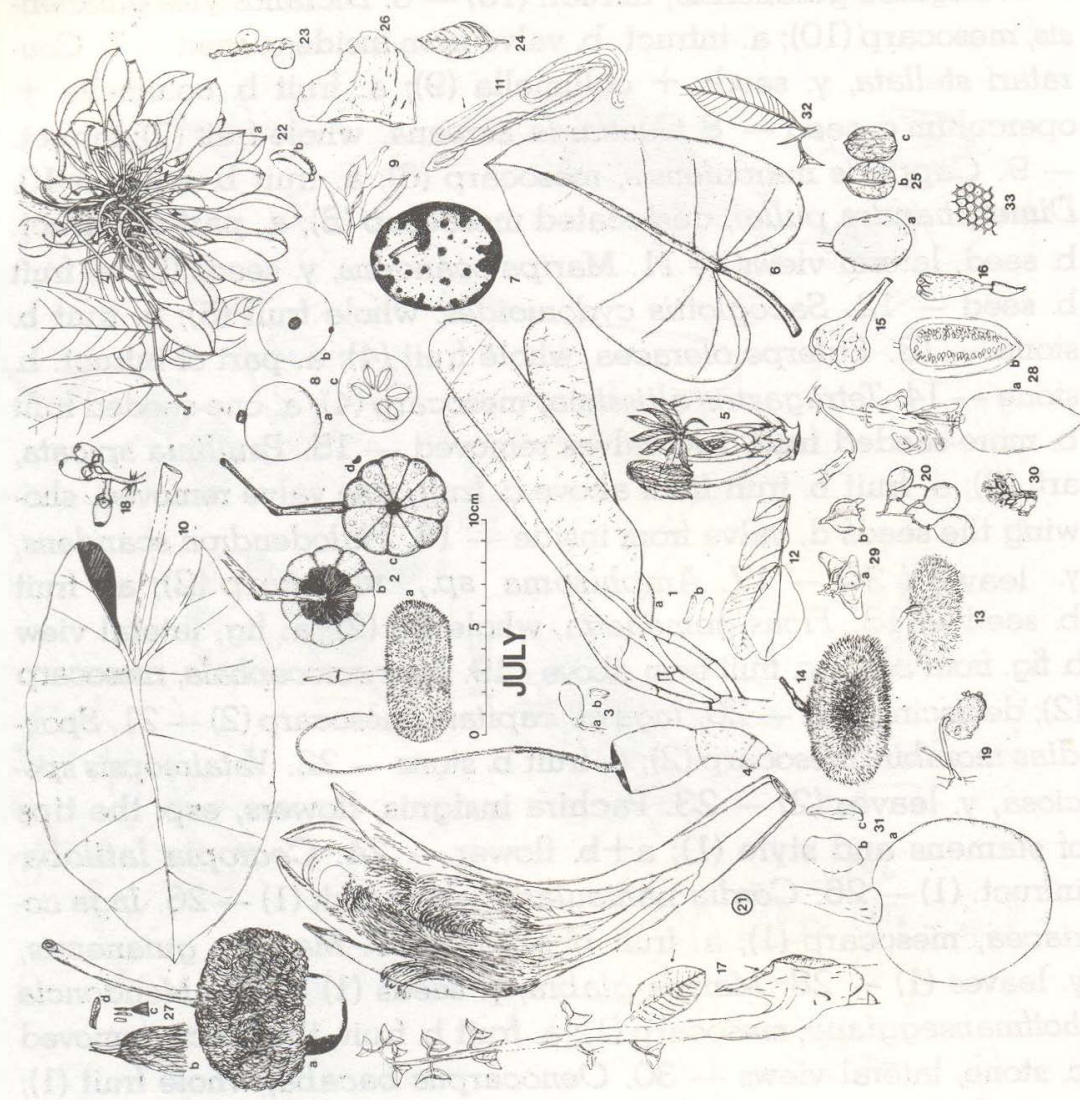

Fig. 32. Diet in July

1. Dimorphandra pullei, desiccated mesocarp (16); a. part of infruct. b. seed, lateral views - 2. Apeiba echinata, mesocarp (6); a. fruit b. fruit from below, the exocarp removed c. fruit from above, the exocarp removed d. seed - 3. Minquartia guianensis, whole fruit (6); a. fruit b. stone - 4. Pachira insignis, flowers, esp. tips of stamens and style (4); $a+b$. flower -5 . Pachira in signis y. leaves (3); part of shoot with y. leaves -6 . Philodendron scandens, y. leaves (3) - 7. Bagassa guianensis, infruct. (2) - 8. Moutabea guianensis, mesocarp (2); $a$. fruit b. fruit from below c. cross section, showing the seeds -9 . Peperomia glabella, y. leaves (2); shoot with y. leaves -10 . Philodendron acutatum, y. lea- 
ves (2) - 11. Philodendron scandens, tips of aerial roots (2) -12 . Pithecellobium jupunba, y. leaves (2) - 13. Apeiba glabra, meso$\operatorname{carp}(1)$; fruit 14. Apeiba schomburgkii, mesocarp (1); fruit - 15. Species a. (Bignoniaceae), flowers (1); corolla - 16. Species b. (Bignoniaceae), flowers (1); flower -17 . Carapa procera, y. leaves + base of petiole (1) - 18. Clusia sp., floral buds (1); floral buds 19. Coussapoa asperifolia, pistillate inflorescences (1); inflor. -20 . Dialium guianense, aril (1); part of infruct.-21. Dioscorea of trifida y. leaves (1); no drawing - 22. Ephedranthus guianensis, mesocarp (1); a. infruct. b. seed - 23. Euterpe oleracea, whole fruit (1); part of infruct. - 24. Hymenolobium petraeum, y. leaves (1); leaflet -25 . Leretia cordata, whole fruit (1); a. fruit b+c. stone, lateral views - 26. Licania micrantha, bark (1) - 27. Parkia nitida, inflor., esp. the stamens and styles of the fertile flowers at the top (1); a. inflor b. sterile flowers c. fertile flowers d. leaflet - 28. Passiflora glandulosa, whole fruit (1); a. fruit b. longitudinal section, showing the seeds - 29. Paullinia tricornis, aril (1); $a+b$. two types of truit - 30. Philodendron scandens, infruct. (1); part of intruct. - 31. Strychnos tomentosa, mesocaxp (1); a. fruit b+c. seed, lateral views -32 . Virola melinonii, y. leaves (1) - 33. Honey (1).

In total: 33 food items

29 tood plant species

71 feeding records

1 honey 


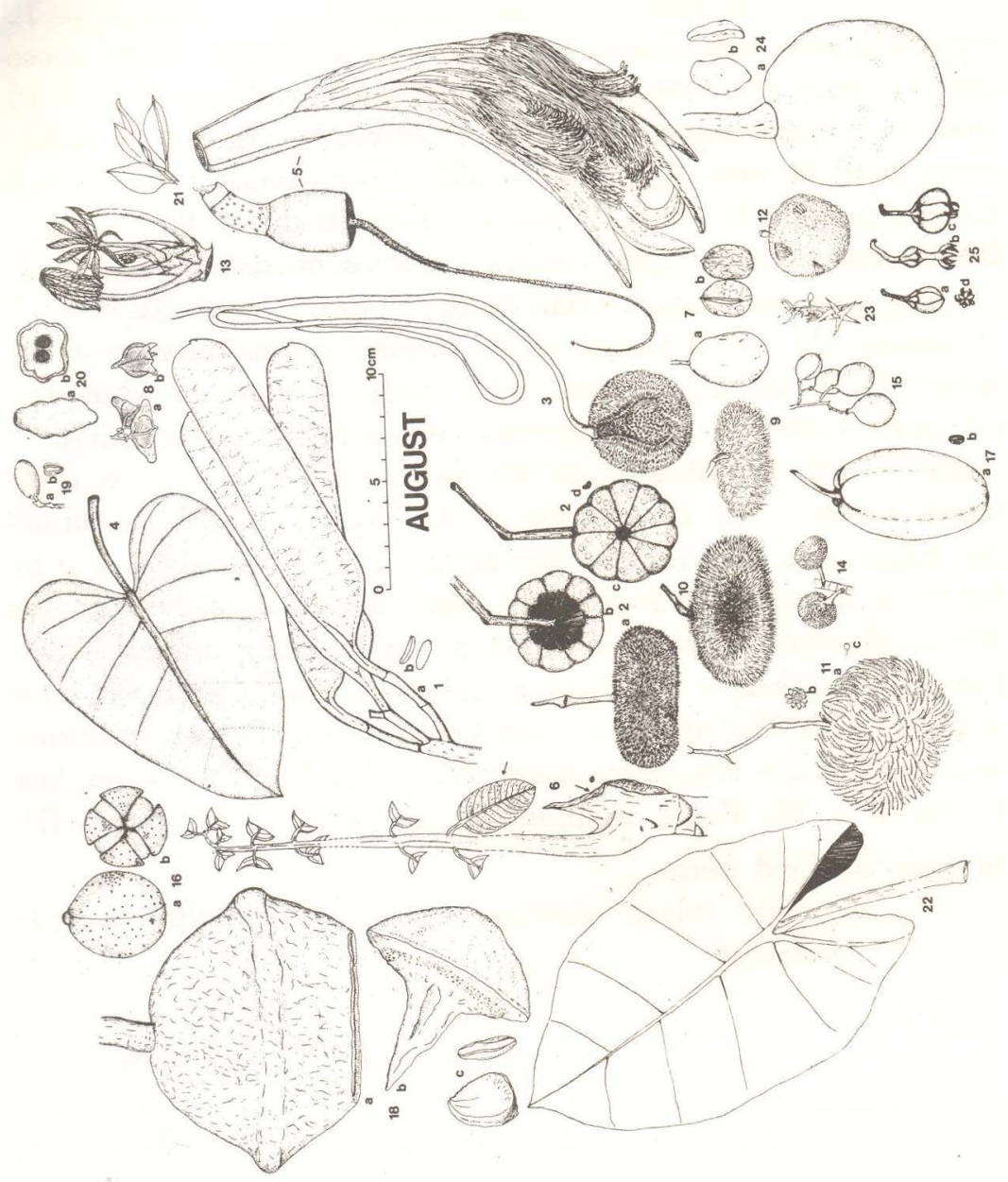

Fig. 33. Diet in August

1. Dimorphandra pullei, desiccated mesocarp (6); a. part of infruct. b. seed, lateral views - 2. Apeiba echinata, mesocarp (5); a. fruit b. truit from below, the exocarp removed c. fruit from above, the exocarp removed d. seed - 3. Parkia pendula, inflor., esp. the tips of flowers (5); inflor., part of the flowers eaten by the monkey - 4. Philodendron scandens, y. leaves (4) - Pachira insignis, flowers (2) - 6. Carapa procera, y. leaves (2) - 7. Leretia cordata, whole fruit (2); a. truit b. stone, lateral views -8 . Paullinia tricomis, aril (2); $\mathrm{a}+\mathrm{b}$. two types of fruit - 9. Apeiba gla- 
bra, mesocarp (1); fruit - 10. Apeiba schomburgkii, mesocarp (1); fruit - 11. Apeiba tibourbou, mesocarp (1); a. fruit b. several seedlets with mesocarp c. seediet - 12. Bagassa guianensis, $y$. infruct. (1) - 13. Pachira insignis, y leaves (1); shoot with young leaves - 14. Coussapoa angustifolia, infruct. (1) - 15, Dialium guianense, aril (1); part of infruct. 16. Guarea kunthiana, aril (1); a. truit b. dehiscing fruit from above - 17. Jacaratia spinosa, whole fruit (1); a. fruit b. seed - 18. Lecythis davisii, mesocarp (1); opened fruit b. operculum c. seed, lateral views - 19. Minquartia guianensis, whole fruit (1); a. fruit b. stone - 20. Parinari excelsa, exocarp + mesocarp (1); a. fruit b. cross section - 21. Peperomia glabella, y. leaves (1) - 22. Philodendron acutatum, y. leaves (1) - 23. Sterculia excelsa, flowers (1); part of inflor. - 24. Strychnos tomentosa, mesocarp (1); a. fruit b. seed, lateral views - 25. Symphonia globulifera, flowers (1); a. floral bud b. flower, the petals removed $c$. flower d. stigma from above.

In total: 25 food items 45 feeding records

24 food plant species 


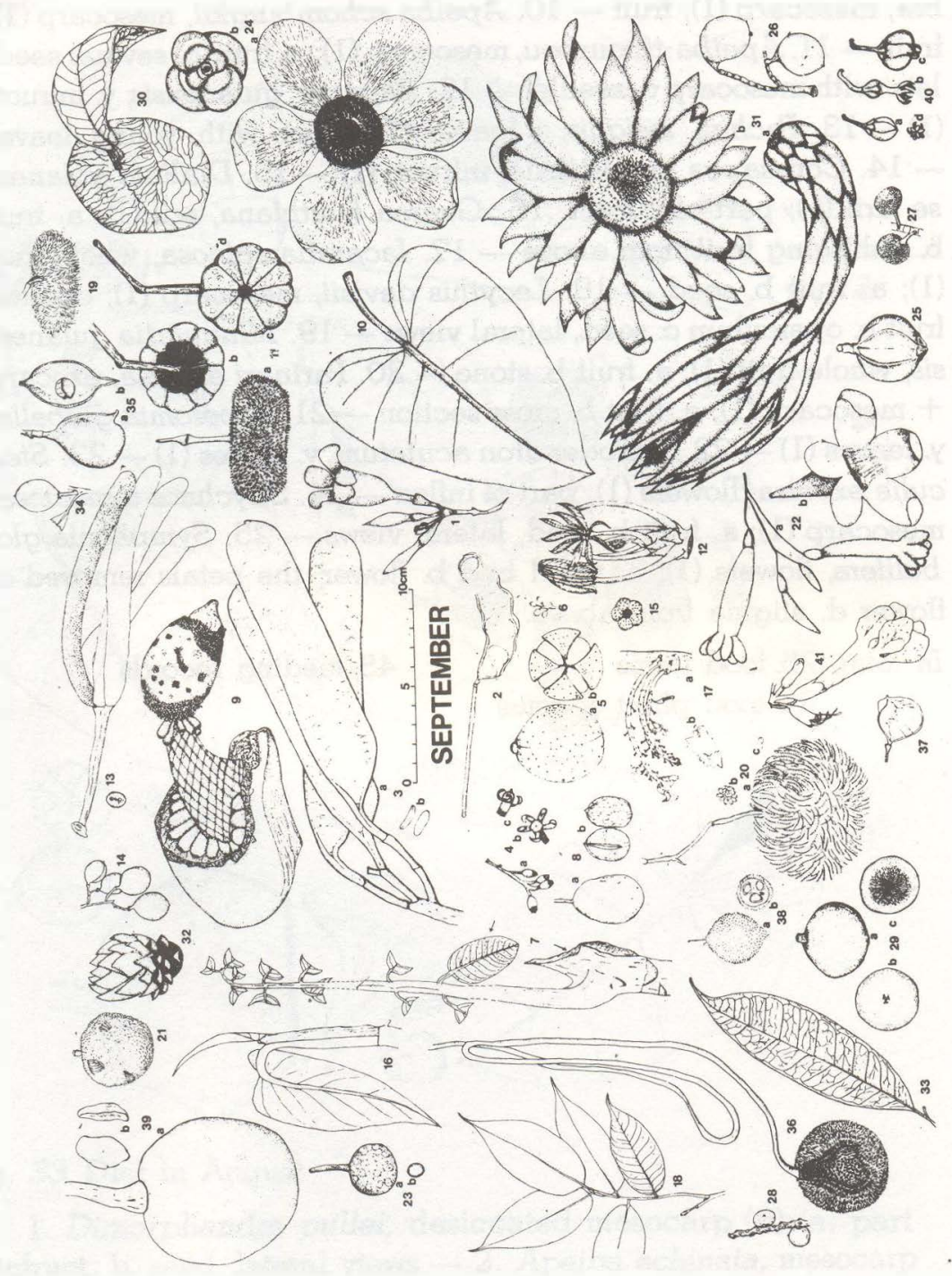

Fig. 34. Diet in September

1. Adenocalymna sp., flowers (11); part of inflor. - 2. Inga leiocalycina, mesocarp (9) - 3. Dimorphandra pullei, desiccated mesocarp (7); a. part of infruct. b. seed, lateral views - 4. Guarea grandifolia, flowers (7); a. part of inflor. with floral buds b. flower from above c. flower, lateral view - 5. Guarea kunthiana, aril (6); 
a. truit b. dehiscing fruit from above -6 . Hyeronima laxiflora, whole fruit (5); part of infruct. - 7. Carapa procera, y. lear... + base of petiole (3) - 8. Leretia cordata, whole fruit (3); a. truir b. stone, lateral views - 9. Philodendron acutatum, intruct. (3); intruct., most of the fruits eaten by the monkey - 10. Philodendron scandens, y. leaves (3) - 11. Apeiba echinata, mesocarp (2); a. fruit b. fruit from below, the exocarp removed $c$. fruit from above, the exocarp removed $d$. seed -12 . Pachira insignis, y. leaves (2); shoot with y. leaves - 13. Cecropia sciadophylla, infruct. (2) - 14. Dialium guianense, aril (2); part of infruct. - 15. Guazuma ulmifolia, whole truit or mesocarp (2); fruit from above -16 . Heteropsis jenmanii, tips of shoots $+\mathrm{y}$. leaves (2); shoot with y. leaves -17 . Platymiscium ulei, flowers (2); a. inflor. b. flower - 18. Pterocarpus officinalis, y. leaves (2); y. leaf - 19. Apeiba glabra, mesocarp (1); truit - 20. Apeiba tibourbou, mesocarp (1); a. fruit b. several seeds with some mesocarp $c$. seed - 21. Bagassa guianensis, y. intruct. (1) - 22. Arrabidaea corallina, flowers (1); a. part of y. inflor. b. opened corolla, obliquely from above -23 . Brosimum parinarioides, infruct. (= pseudofruit) (1); a. infruct. b. seed -24 . Clusia platystigma, male flowers, esp. central body of staminodes (1); a. flower from above, showing the central body of staminodes b. flower from below, the petals removed, showing bracteola and sepals - 25. Clusia purpurea, pulp (= many seedlets with aril) (1); fruit - 26. Copaifera epunctata, aril (1); infruct. - 27. Coussapoa angustifolia, infruct. (1) -28 . Euterpe oleracea, whole fruit (1); part of infruct. -29 . Ficus insipida, whole fig (1); $a$. fig $b$. fig from above c. cross section 30. Helicostylis pedunculata, infruct. (1); infruct., one leaf attached - 31. Hylocereus sp. a., flowers, esp. tips of stamens and style, and stigma (1); a. flower b. flower from above 32. Hylocereus sp. b., flowers, esp. the tips (1); flower, the tip eaten by the monkey - 33. Maquira guianensis, y. leaves (1) - 34. Micropholis guyanensis, mesocarp - 35. Ormosia paraënsis, y. seeds (1); a. fruit b. fruit, one valve removed, showing the mir:etic seed -- 36. Parkia pendula, inflor, esp. the tips of flowers (1); inflor. with part of peduncle, part of the flowers eaten by the monkey - 37 . Peltogyne venosa, aril (1); fruit -38 . . Rheedia benthamiana, aril (1); a. fruit b. cross section showing the seeds -39 . Strychnos tomentosa, mesocarp (1); a. fruit b. seed, lateral views - 40. Symphonia globulifera, flowers (1); $a$. floral bud $b$. flower, the petals removed 
c. flower d. stigma from above - 41. Tabebuia serratifolia, flowers (1); part of inflor, the corolla of one flower eaten by the monkey.

In total 41 food items

96 feeding records

41 food plant species 


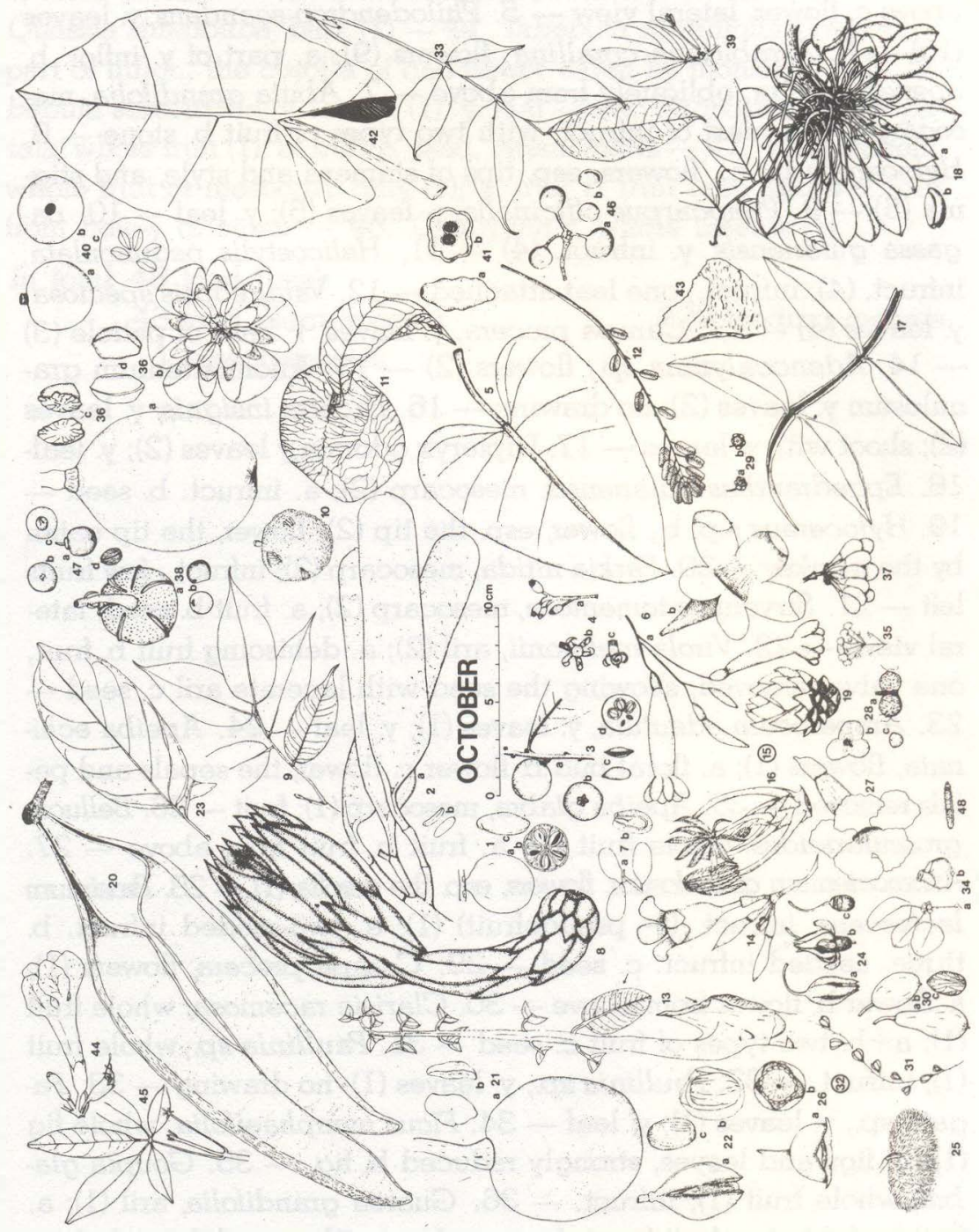

Fig. 35. Diet in October

1. Ecclinusa guianensig mesocarp (71); a. fruit b. cross section, showing the seeds - 2. Dimorphandra pullei, desiccated mesocarp (24); a. part of infruct. b. seed, lateral views - 3.. Achrouteria pomifera, mesocarp (17); a. fruit b. fruit from below c. seed, lateral views $d$. cross section, showing the seeds -4 . Guarea gran- 
difolia, flowers (11); a. part of inflor, with floxal buds b. flower from above $c$. flower, lateral view - 5. Philodendron scandens, $y$. leaves (10) - 6. Arrabidaea corallina, flowers (9); a. part of y. inflor. b. opened corolla, obliquely from above - 7. Abuta grandifolia, mesocarp (7); a. part of infruct, with two types of fruit b. stone -8 . Hylocereus sp. a., flowers, esp. tips of stamens and style, and stigma (5) - 9. Pterocarpus officinalis, y. leaves (5); y. leaf -10 . Bagassa guianensis, y. infruct. (4) - 11. Helicostylis pedunculata, infruct. (4); infruct., one leaf attached - 12. Vataireopsis speciosa, $y$. leaves (4) - 13. Carapa procera, y. leaves + base of petiole (3) - 14. Adenocalymna sp., flowers (2) - 15. Anomoctenium granulosum y. leaves (2); no drawing - 16. Pachira insignis, y. leaves (2); shoot with y. leaves - 17. Dipteryx odorata y leaves (2); y. leaf18. Ephedranthus guianensis, mesocarp (2); a. infruct. b. seed 19. Hylocereus s.p. b., flower, esp. the tip (2); flower, the tip eaten by the monkey -20 . Parkia nitida, mesocarp (2); infruct., few fruits left -21 . Strychnos tomentosa, mesocarp (2); a. fruit b. seed, lateral views -22 . Virola melinonii, aril (2); a. dehiscing fruit b. fruit, one valve removed, showing the seed with lacerate aril c. seed 23. Ampelocera edentula, y. leaves (1); y. leaf -24 . Apeiba echinata, flowers (1); a. floral bud b. flower c. flower, the sepals and petals removed - 25. Apeiba glabra, mesocarp (1); fruit -26. Bellucia grossularioides, whole fruit (1); a. fruit $b$. fruit from above -27 . Anomoctenium granulosum, flowers, esp the conolla (1) - 28. Brosimum lactescens, infruct. ( $=$ pseudofruit) (1); a. two-seeded infruct. b. three-seeded infruct. c. seed - 29. Carapa procera, flowers (1); a. flower $b$. flower from above - 30. Clarisia racemosa, whole fruit (1); $a+b$. two types of fruit c. seed - 31. Paullinia sp., whole fruit (1); infruct. - 32. Paullinia sp., y. leaves (1); no drawing - 33. Fagara sp., y. leaves (1); y. leaf - 34. Ficus nymphaeifolia, whole fig (I); a. figs and leayes, strongly reduced b. fig. - 35. Goupia glabra, whole fruit (1); infruct. - 36. Guarea grandifolia, aril (1); a. fruit $b$. dehiscing fruit from above c. stone without aril, lateral views d. stone with aril, lateral views - 37. Guatteria chysopetala, whole fruit (1); infruct. -38 . Gustavia hexapetala, mesocarp + funicle (1); a. fruit from below b. seed, lateral views - 39. Not identified species (Loranthaceae), y. leaves (1) - 40. Moutabea guianensis, mesocarp (1); $a$. fruit $b$. fruit from below c. cross section, showing the seeds - 41. Parinari excelsa, exocarp + mesocarp (1); a. fruit 
b. cross section -42 . Philodendron acutatum, y. leaves (1) -43 , Quassia simarouba, bark (1) - 44. Tabebuia serratifolia, flowers (1); part of inflor, the corolla of one llower eaten by monkey -45 . Tabebuia serratifolia, y. leaves (1); y. leaf - 46. Unonopsis glaucopetala, whole fruit (1); a. fruit b. seed, lateral views - 47. Vitex stahelii, whole fruit or mesocarp only (1); $a$. fruit $b$. fruit from above c. fruit trom below d. stone - 48. Caterpillars, whole insects ( 1 )

In total 48 food items

43 food plant species

222 feeding records

1 insect species 


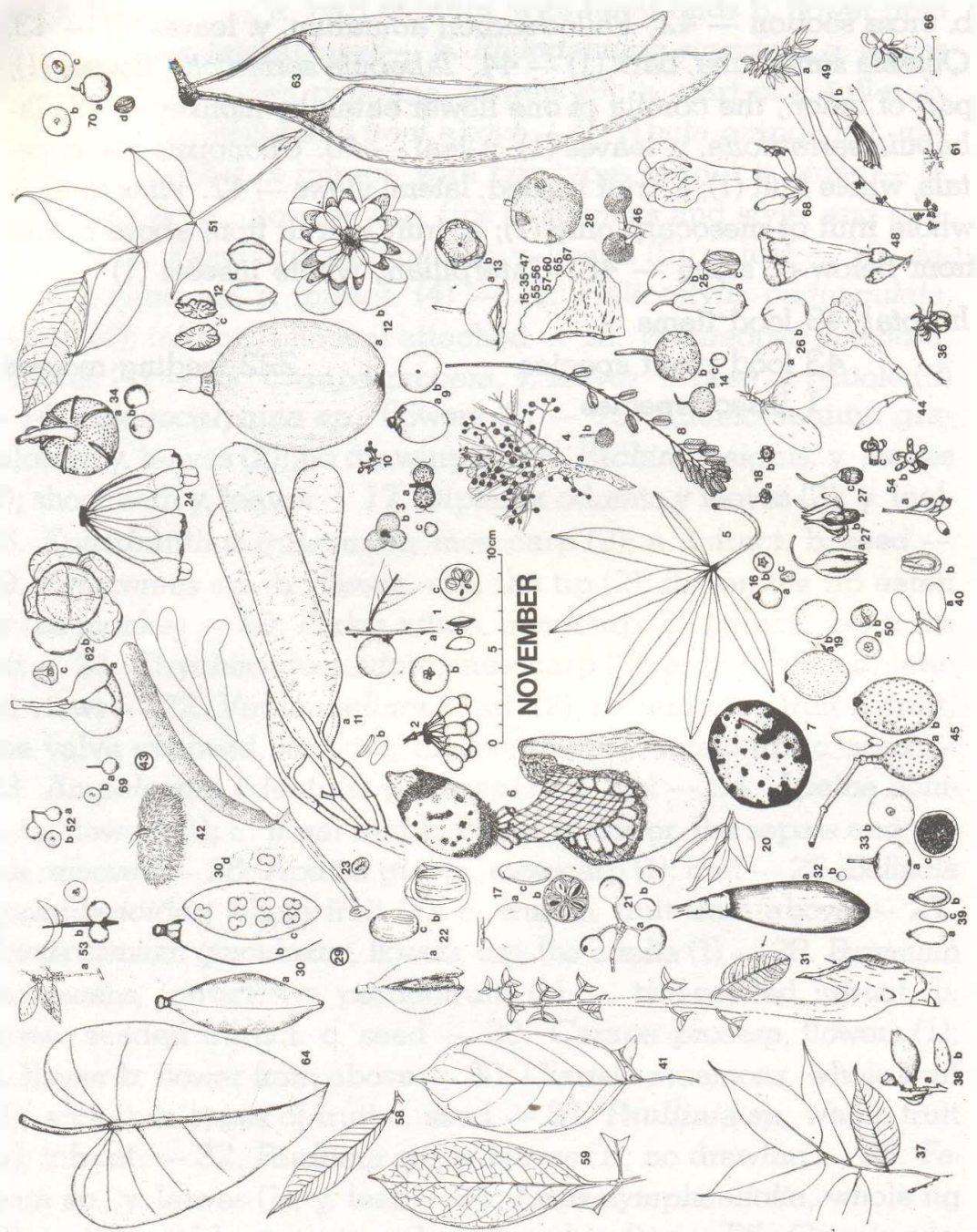

Fig. 36. Diet in November

1. Achrouteria pomifera, mesocarp (31); a. fruit b. fruit from below c. cross section, showing the seeds d. seed, lateral views -2 . Guatteria chrysopetala, whole fruit (18); infruct. - 3. Brosimum lactescens, infruct. (11); a. two-seeded infruct. b. three-seeded infruct. c. seed - 4. Coussapoa latifolia, infruct. (11); a. twig infruct., strongly reduced b. infruct. - 5. Ceiba pentandra, y. leaves (10); y. leaf, the apical part of the leaflets eaten - 6. Philodendron acutatum, 
infruct. (10); part of intruct., most of the fruits eaten by the monkey - 7. Bagassa guianensis, intruct. (9) - 8. Vataireopsis speciosa, y. leaves $(3)-9$. Ficus gardneriana, whole fig $(8)$; a. tig b. from above - 10. Ampelocera edentula, flowers (7); part of inflor. - 11 . Dimorphandra pullei, desiccated mesocarp (7); a. part of intruct. b. seed, lateral views - 12. Guarea grandifolia, aril $(7)$; $a$. fruit $b$. dehiscing fruit from above $c$. stone without aril, lateral views $d$. stone with aril, lateral views - 13. Bellucia grossularioides, whole truit (6); a. fruit b. fruit from above - 14. Brosimum parinarioides, infruct. (6); a. y. infruct. b. mature infruct. c. seed - 15. Licania micrantha, bark (6) - 16. Cordia sagotii, whole fruit (5); a. fruit b. truit trom below c. stone - 17. Ecclinusa guianensis, mesocarp (5); a. fruit $b$. cross section, showing the seeds c. seed - 18. Carapa procera, flowers (4); a. flower b. flower from above - 19. Couepia caryophylloides, exocarp + mesocarp (4); a. fruit b. stone -20 . Eeperomia glabella, y. leaves (4) - 21. Unonopsis glaucopetala, whole fruit (4); a. infruct. b. seed, lateral views -22 . Virola melinonii, aril $(4) ; a$. just dehiscing truit $b$. fruit, one valve removed $c$. seed with aril - 23. Cecropia sciadophylla, intruct. (3) -24 . Ceiba pentandra, floral buds + flowers (3); inflor. -25 . Clarisia racemosa, whole fruit (3); $a+b$. two types of truit c. seed -26 . Gnetum unens, perigonium or whole fruit (3); a. infruct. b. seed-27. Apeiba echinata, flowers (2); a. floral bud b. flower c. flower, the sepals and petals removed -28 . Bagassa guianensis, y. infruct. (2) -29 . Anomoctenium granulosum, y. leaves (2); no drawing - 30. Anomoctenium granulosum, y. seds (2); $a$. truit b suture $\mathrm{c}$ fruit, one valve removed, showing the seeds $d$. seed - 31. Carapa procera, y leaves + base of petiole (2); leaf -32 . Ceiba pentandra, exocarp of y. fruits (2); a. y. fruit b. seed - 33. Ficus broadwayi, whole tig (2); a. fig b. fig from above c. cross section - 34. Gustavia hexapetala, mesocarp + funicle (2); a. fruit from below b. seed, lateral views 35. Inga alba, bark $(2)-36$. Pereskia aculeata, flowers (2) - 37 . Pterocarpus officinalis, y. leaves (2); leaf - 38. Talisia squarrosa, mecocarp (2); $a$. infruct., one fruit eaten out by the monkey b. seed - 39. Trichilia surinamensis, whole fruit $(2)$; $a$. fruit $b$. truit, two valves removed c. seed - 40. Abuta grandifolia, mesocarp (1); a. part of infruct. with two types of fruit b. stone -41 . Ampelocera edentula, y. leaves (1) - 42. Apeiba glabra, mesocarp (1); fruit 43. Attalea maripa, decaying and rotten sheaths (1) -.. 44. Xyloph- 
ragma seemannianum, flowers $(1)-45$. Cedrela odorata, $y$. seeds + columella (1); a. y. fruit b. mature fruit c. seed - 46. Coussapoa angustifolia, infruct. (1) - 47. Dimorphandra pullei, bark (1) - 48. Stizophyllum inaequilaterum, flowers (1) - 49. Eperua falcata, flowers (1) - 50. Euterpe precatoria, whole fruit (1); a. fruit from below b. stone - 51. Zanthoxylum rhoifolium. (1); leaf - 52. Ficus malacocarpa, whole fig (1); a. infruct. b. fig from above - 53. Ficus trigona, whole fig (1); a. twig infruct., strongly reduced b. infruct. c. fig. from above - 54. Guarea grandifolia, flowers (1); a. part of inflor. b. flower from above c. flower, lateral view - 55. Hyeronima laxiflora, bark (1) - 56. Inga leiocalycina, bark (1) - 57. Licania majuscula, bark (1) - 58. Licania micrantha, y. leaves (1) - 59. Licaria canella, y. leaves (1) - 60 . Nectandra kunthiana, bark (1) - 61. Norantea guianensis, flowers, esp. the honeycups (1); part of inflor. 62. Operculina hamiltoni, y. seeds (1); a. floral bud b. flower from above c. seed 63. Parkia nitida, mesocarp (1); intruct., two fruits left -64 . Philodendron scandens, y. leaves (1) - 65. Pithecellobium jupunba, bark (1) - 66. Qualea dinizii, flowers (1); flower - 67 . Sacoglottis cydonioides, bark (1) - 68. Tabebuia capitata flowers (1); part of inflor. -69 . Trymatocaccus oligandrus, whole fruit (1); a. fruit b. fruit from above -70 . Vitex stahelii, whole fruir or mesocarp only (1); $a$. fruit $b$. fruit from above. c. fruit from below d. stone.

In totak 70 food items 255 feeding records

45 food plant species 


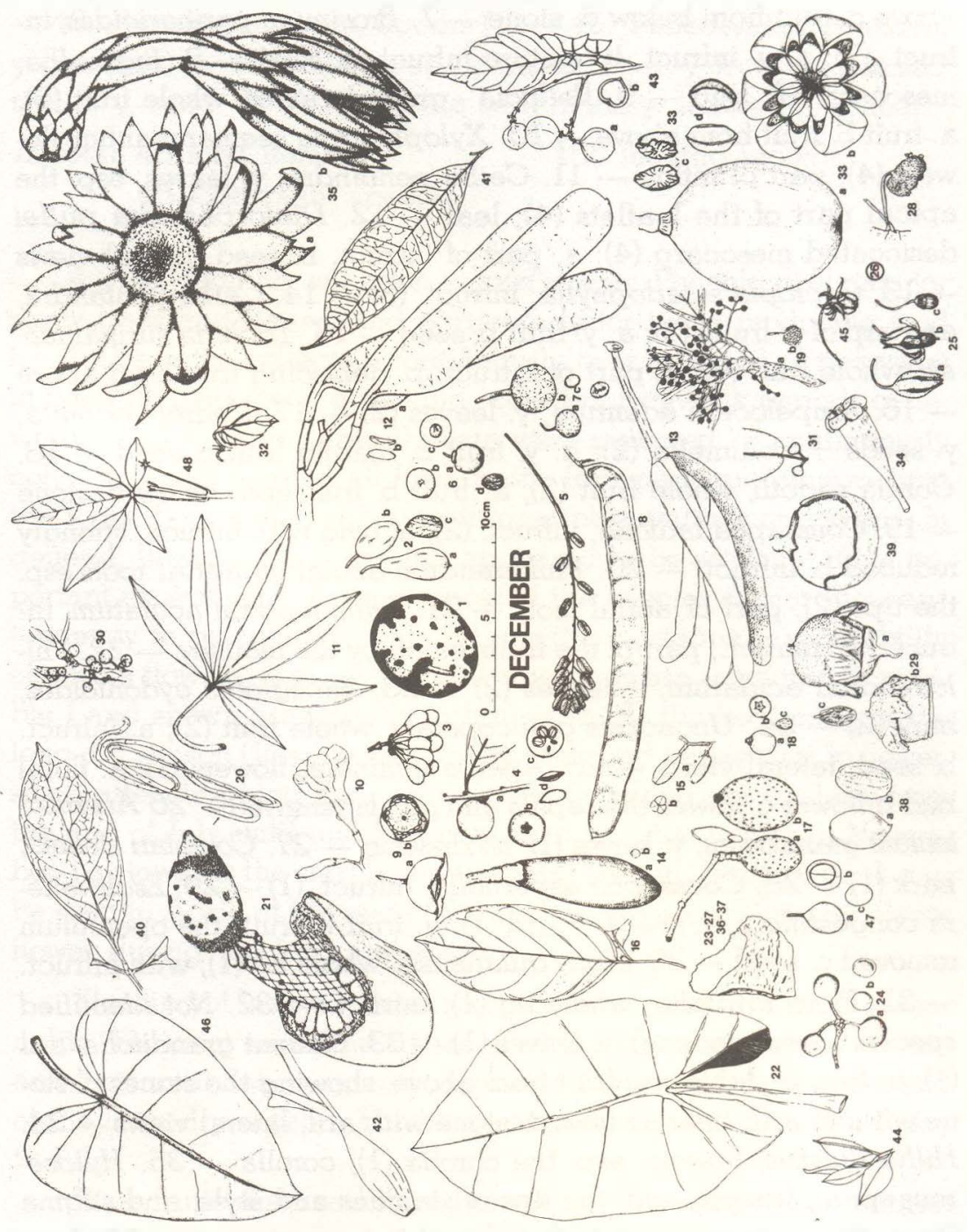

Fig. 37. Diet in December

1. Bagassa guianensis infruct. (20) -2 . Clarisia racemosa, who le fruit (14); $a+b$. two types of fruit c. seed - 3. Guatteria chrysopetala, whole fruit (13); intruct. - 4. Achrouteria pomifera, mesocarp (11); a. fruit b. from below c. cross section, showing the seods d. seed, lateral views -5 . Vataireopsis speciosa, y. leaves (9) -6 . 
Vitex stahelii, whole fruit or mesocarp only (8); $a$. fruit b. fruit from above c. truit from below d. stone - 7. Brosimum parinarioides, infruct. (7); a. y. infruct. b. mature infruct. c. seed - 8. Inga alba, mesocarp (6); fruit - 9. Bellucia grossulariodes, whole fruit (5); a. fruit $b$. fruit from above -10 . Xylophragma seemannianum, flowers (4); part of inflor. - 11. Ceiba pentandra, y. leaves, esp. the apical part of the leaflets (4); leaf - 12. Dimorphandra pullei desiccated mesocarp (4); a. part of infruct. b. seed, lateral views - 13. Cecropia sciadophylla, infruct. (3) - 14. Ceiba pentandra, exocarp of y. fruits ( 3 ); a. y. fruit b. seed - 15. Trichilia surinamensis, whole truit (3); a. part of infruct. b. dehiscing fruit from above - 16. Ampelocera edentula, y. leaves (2) - 17. Cedrela odorata, y. seeds + columella (2); a. y. fruit b. mature fruit c. seed - 18 . Cordia sagotii, whole fruit $(2) ; a$. fruit $b$. fruit from below $c$. stone - 19. Coussapoa latifolia, infruct. (2); a. twig with infruct., strongly reduced $b$. infruct. -20 . Philodendron acutatum, aerial roots esp. the tips (2); part of aerial root, -21 . Philodendron acutatum, infruct. (2); infruct., part of the fruits eaten by the monkey. -22 . Philodendron acutatum, y. leaves (2) - 23. Sacoglottis cydonioides, bark (2) - 24. Unonopsis glaucopetala, whole fruit (2); a. infruct. b. seed, lateral views - 25. Apeiba echinata, flowers (1); a. floral bud b. flower c. flower, the sepals and petals removed - 26 Anamoctenium granulosum, y. leaves (1); no drawing - 27. Couratari stellata, bark (1)-28. Coussapoa asperifolia, infruct. (1) - 29. Eschweilera congestiflora, y. seeds + aril (1); $a$. fruit $b$. fruit, the operculum removed c. seed - 30. Ficus guianensis, whole fig (1); with infruct. -31 . Ficus citrifolia, whole fig (1); infruct. - 32. Not identified species (Gesneriaceae), y. leaves (1) - 33. Guarea grandifolia, aril (1); a. fruit b. dehiscing fruit from above, showing the stones $c$. stone without aril, lateral views d. stone with aril, lateral views -34 . Hillia illustris, flowers, esp. the corolla (1); corolla -. 35. Hylocereus sp. a., flowers, esp. the tips of stamens and style, and stigma (1); a. flower from above b. flower just before opening - 36. Inga alba, bark (1) - 37. Inga kiccalycina, beuk (1) - 38. Vantanea parviflara, exocarp + mesocarp (1); a. fruit b. stone - 39. Licania majuscula, exocarp + mesocarp (1); fruit, the exocarp and mesocarp partly scraped ot the monkey - 40. Licaria canella, y. leaves (1) - 41. Maquira guianensis, y. leaves (1) - 42. Species b. (Orchidaceae), pseudobulbs (1) - 43. Paullinia sphaerocarpa, y. seeds (1); a. in- 
fruct. b. valve from inside -44 . Peperomia glabella, y. leaves ( 1 ) - 45. Pereskia aculeata, flowers (1) - 46. Philodendron scandens y. leaves (1) - 47. Pouteria sp., y. seeds (1); a. y. truit b. cross section c. y. seed -48 . Tabebuia serratifolia y. leaves (1); y. leaf.

In total 48 food items

155 feeding records

45 food plant species

4.2.10. Monthly variation in food supply. Phenological data for many trees and lianes, and particularly for those producing food for Ateles, were collected continuously over a period of two years in the Voltzberg study area, and scored in two-week periods of flowering and fruiting (table I). Data were gathered by continuously collecting and naming all fresh fruits, fruit parts and flowers from the forest floor and making additional observations on crowns. In general, flowering and fruiting appeared to be seasonal. One important exception is Ficus, as most of the species do not show any tendency to seasonality. Data on monthly variation in availability of edible flowers, flush leaves and ripe fruits are extracted from table $I$ and shown graphically in figure 38. At the beginning of the long dry season (July/August), many trees and lianes start to flower, some after dropping their leaves. The flowering season lasts from the end of July until mid-February with a distinct peak in November, i.e., towards the start of the short wet season. A minor peak can be seen in April, at the end of the short dry season. Very few plants flower during the long wet season.

Flushing of leaves follows a somewhat similar pattem of seasonality. For most trees and lianes, flushing starts at the end of July and lasts until February, with a peak in November towards the end of the long dry season. Flushing seems to be rare from February to July.

Ripe fruits are available throughout the year, but a distinct peak can be seen in the months of March, April and May. Towards the end of the long wet season the availability of ripe fruits drops drastically, reaching a minimum in July and Augus ${ }^{+}$. A minor peak canbe seen in November at the start of the short wet season.

Most tree species tend to fruit every year and, at an individual level, usually alternate a good cxop with a minor one. However, the timing of flowering and fruiting in a particular species can vary con- 
siderably trom year to year, perhaps because of yearly variations in timing and duration of the different seasons and/or differences in rainfall. A shift of one to two months and sometimes even more is not unusual (e.g., Pachira insignis was flowering in 1977 trom mid-June to mid-August, and in 1978 from March to May). Moreover, individuals of some species seem to fruit only once every two

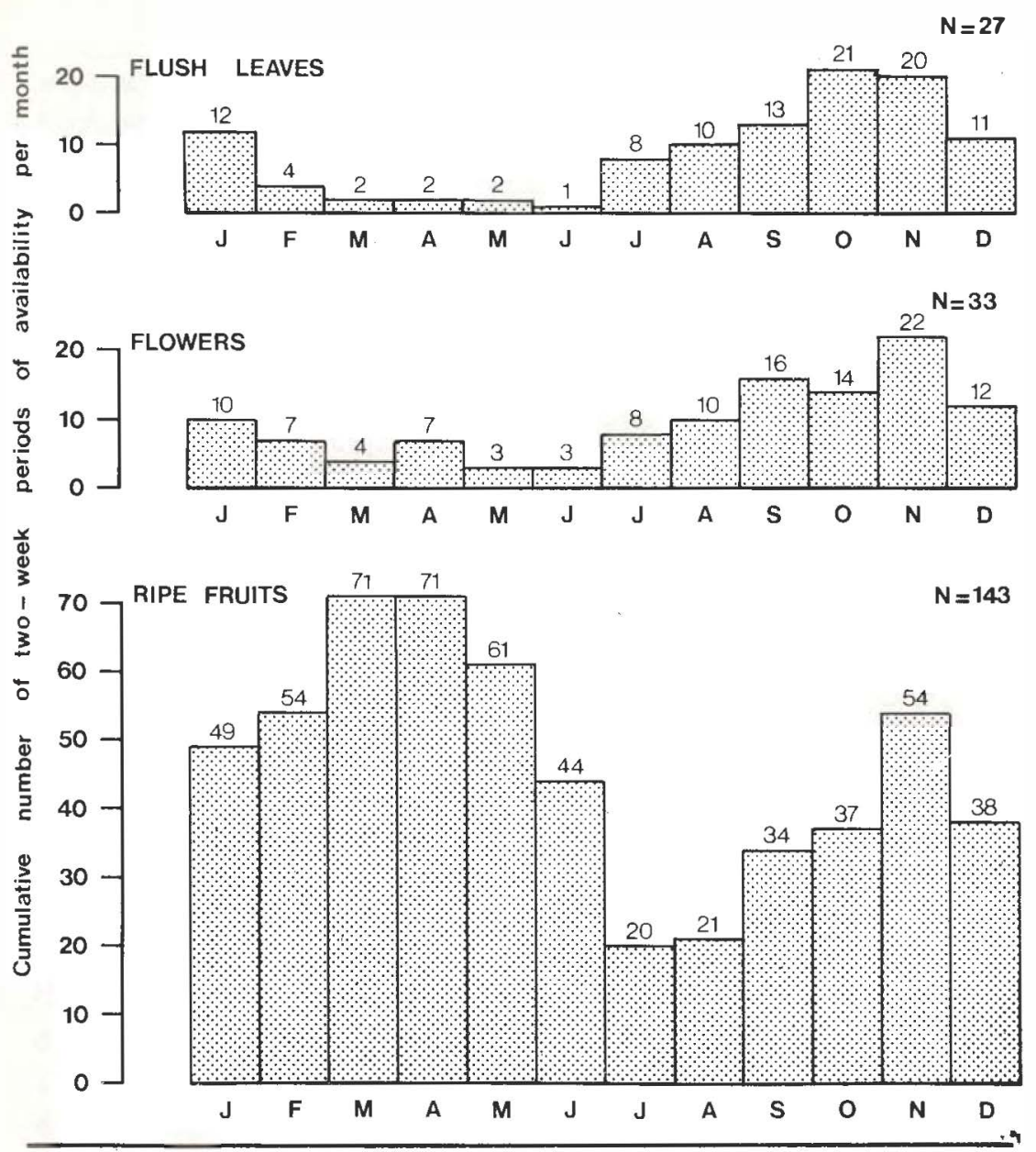

Figure 38 Monthly variation in availability of edible flowers, flush leaves and ripe fruits for spider monkeys in the Voltzberg study area during the present study. Data are expreseed as the sum of two-weel periods in which the itams wers availabla Only those species were taten into account for which phenological data were collected systematically throughout the year (33 specis of flower, 27 species of leaf and 143 species of truit; 800 table D. 
years, or even once every three to four years (e.g., Hymenolobium). Some years the whole fruit crop of a species may fail for some reason (e. g., Minquartia guianensis in 1977; Capparis maroniensis in 1978). Many lianes seem to fruit without any tendency to seasonality (e.g., Strychnos, Ficus, Moutabea guianensis), and some trees (e.g., Guarea grandifolia, Ficus) and lianes (e.g., Ficus, Abuta grandifolia) fruit about twice a year.

In some species, fruits are present for prolonged periods (e.g., Capparis maroniensis, 4 1/2 months; Dimorphandra pullei, 7 months; Licania majuscula, 5 months; Virola melinonii, 5 l/2 months) since fruiting is not synchronized at an individual level. Other species provide the monkeys with ripe fruits for only 10-14 days (e.g., Ampelocera edentula, several Ficus species.).

4.2.11. Food supply and food choice. Table IX compares the monthly supply of food items with the food choice observed. Data are extracted from Table I and Figures 26-37. Food items not included are bark, decaying wood, pseudobulbs, honey and insects because their availability seems to be either permanent or hard to determine. From June to October, during the end of the long wet season and the first part of the long dry season, food supply is lowest and the monkeys seem to exploit just about all available food items. In contrast, during December and January in particular, a food excess seems to exist in terms of percentages of food items not exploited (17.3 and $15.0 \%$, respectively). The foods neglected during December are mainly flowers, and during January, they are young seeds and low-nutrient fruits of minor overall importance. However, when all food items are considered to be equally preferred by spider monkeys, comparing food supply and food choice in this way does not provide information about the real food excess. Spider monkeys prefer ripe fruits over all other foods. In Table X, the food supply is expressed both as the total number of two-week periods of availability of fruits and as the total number of potential edible-fruit bearing plants in the area for each month of the year. During December and January, the months apparently providing the monkeys with an excess of food items (Table IX), the supply of preferred ripe fruits is still rather low. This forces the monkey to eat considerable amounts of young leaves and flowers (Fig. 25). In contrast, in the period March-July (and in April, May and June in par- 
ticular) preferred food species obviously provide the monkeys with an excess of ripe fruits. This results in enormous amounts of spontaneously-falling unexploited fruits when species such as Guarea grandifolia, Tetragastris altissima, Protium polybotryum, Capparis, maroniensis, Spondias mombin and Virola melinonii are producing large crops. In this period the monkeys eat very low percentages of leaves and flowers (Fig. 25). The supply of fruits and young seeds is extremely low in the period July-November, in July and August in particular. This is obviously a period of food scarcity for the spider monkeys. When crops of certain species fail (as was the case in 1976), the monkeys suffer from food shortage.

Table IX. Food supply compared with food choice for each month of the year, expressed as the total number of food items the food excess is expressed as the percentage of food items not exploited. Not included are bark, decaying wood, pseudobulbs, honey and insects.

\begin{tabular}{|c|c|c|c|}
\hline \multirow[t]{2}{*}{ Month } & \multicolumn{2}{|c|}{ Total number of food items } & \multirow{2}{*}{$\begin{array}{l}\% \text { Food items } \\
\text { not exploited }\end{array}$} \\
\hline & available & eaten & \\
\hline January & 53 & 45 & 15.0 \\
\hline February & 47 & 42 & 106 \\
\hline March & 49 & 48 & 2.0 \\
\hline April & 54 & 51 & 36 \\
\hline May & 51 & 47 & 78 \\
\hline Juine & 32 & 32 & 0,0 \\
\hline July & $\$ 1$ & 31 & 00 \\
\hline August & 24 & 24 & 0.0 \\
\hline September & 39 & 38 & 26 \\
\hline October & 50 & 46 & 80 \\
\hline November & 66 & 60 & 9.9 \\
\hline December & 52 & 43 & 17.3 \\
\hline
\end{tabular}

\subsection{Ateles as a Specialized Frugivore}

Among frugivores, three major categories are distinguishable in relation to fruits and seeds eaten:

1. Total frugivores (Morton, 1973), specialized trugivores (McKey, 1975) or specialists

2. Partial frugivores (Morton, 1973), opportunistic frugivores (McKey, 1975) or generalists

3. Seed-eaters or seed-predators.

Ateles can be considered one of the most prominent specialized frugivores in the Neotropics. Specialists are best characterized 
Table X. Food supply tor a group of spider monkeys in the Voltzberg study area, expressed as the comulative number of two-week periods of availability for fruits $(A)$, young seeds $(B)$ and both $(A+B)$, and as the total number of potential ripefruit sources $(C)$, young-seed sources (D) and both $(C+D)$ for each month of the year. Data on availability and numbers of potential tood sources are extracted from Table I.

Month.

Total number of two-week pe- Total number of fruit sources riods of availability for for

\begin{tabular}{|c|c|c|c|c|c|c|}
\hline & & \\
\hline & $\begin{array}{c}\text { ipe truits } \\
\text { A }\end{array}$ & $\begin{array}{c}\text { young seeds } \\
\text { B }\end{array}$ & $\begin{array}{l}\text { truits } \\
A+B\end{array}$ & $\begin{array}{c}\text { ripe frists } \\
\text { C }\end{array}$ & $\begin{array}{c}\text { poung seeds } \\
\text { D }\end{array}$ & $\begin{array}{l}\text { truits } \\
C+D\end{array}$ \\
\hline January & 49 & 19 & 68 & 412 & 429 & 841 \\
\hline February & 54 & 9 & 63 & 480 & 213 & 693 \\
\hline March & 71 & 7 & 78 & 641 & 209 & 850 \\
\hline April & 71 & 3 & 74 & 1,298 & 344 & 1,642 \\
\hline May & 61 & 12 & 73 & 1,213 & 719 & 1,932 \\
\hline June & 44 & 5 & 49 & 1,086 & 205 & 1,291 \\
\hline July & 20 & 0 & 20 & 175 & 0 & 175 \\
\hline August & 21 & 1 & 22 & 143 & 6 & 149 \\
\hline September & 34 & 2 & 36 & 200 & 12 & $2 \mathrm{I} 2$ \\
\hline October & 37 & 2 & 39 & 253 & 12 & 265 \\
\hline November & 54 & 3 & 57 & 487 & 7 & 494 \\
\hline December & 38 & 10 & 48 & 364 & 23 & 387 \\
\hline
\end{tabular}

as animals that derive most of their supplies of carbohydrate, lipid and protein from fruits throughout the year. They, therefore, are restricted to the tropics. In the Neotropics several cotingids (e.g., the bellbird, the cock-ot-the-rock), the oilbird, several trogons (e.g., the quetzal), guans, most toucans, kinkajous, howler monkeys, woolly monkeys (Lagothrix spp.) and woolly spider monkeys (Brachyteles arachnoides) also belong to this category.

Generalists are characterized as animals that mainly utilize fruits as a quickly harvestable source of carbohydrates, organic acids, water and possibly minerals that does not interfere much with the harvesting of proteins and lipids from other resources such as insects and meat (Morton, 1973). Generalists may be primarily or partial insectivorous or camivorous. The majority of Neotropical birds and monkeys belong to this cateqory, among them trumpeters, curassuws, chachalacas, quails, tinamous, woodpeckers, caciques, tanagers, oropendulas, orioles, manakins, trushes, woodwarblers, tyrant flycathers, squirrel monkeys, capuchin monkeys and callitrichid monkeys. Other mammals such as marsupials, coatis, raccoons and tayras also fall into this category. 
Seed-eaters derive most of their supplies of lipid and protein from seeds eaten in a young or mature stage. Pre-dispersal (arboreal) seed predation is performed especially by doves, grosbeaks, buntings, sparrows, finches, parakeets, parrots, macaws, tree porcupines, squirrels and saki monkeys (Chiropotes and Pithecia), whereas post-dispersal (terrestrial) seed predation is performed most notably by rodents such as acouchis, agoutis, pacas, spiny rats and nice rats. Among the insects, bruchid weevils are notorious seed predators.

In general, fruits eaten by specialists are characterized by a firm, dense edible part (aril or mesocarp) surrounding the seed or stone and containing a relatively high portion of fat and protein, and also by having a single or only few large seeds (e.g., Virola, Ocotea, Oenocarpus, Protium, Tetragastris). In contrast, fruits ingested by a wide variety of generalists generally have juicy, watery flesh and contain many small seeds (e.g., Carica, Cecropia, Ficus, Jacaratia, Loreya, Miconia, Passiflora). Fruits predated for the mature seeds after dispersal are usually dry, have only a minuscule aril attached to the seed, or else have a thin layer of mesocarp surrounding the seed or stone. The seeds are in some way protected against pre-dispersal predation by a thick, woody, dehiscent or indehiscent exocarp or by a tough, bony or woody endocarp, (e.g., Andira, Astrocaryum, Attalea, Bertholletia, Carapa, Couepia, Eschweilera, Lecythis, Licania, Mora, Pachira, Vouacapoua).

By means of comparative morphological analysis of 1,567 different fruits of Surinam woody plants above $1.5 \mathrm{~m}$ in height, Van Roosmalen (in prep.), estimates that dispersal by wind (anemochory) takes place in $10.6 \%$ of the species, by water in $5.2 \%$, by the plant itself (autochory) in $0.3 \%$, by specialists and scatter-hoarding rodents in $41.9 \%$ and by generalists (together with specialists) in $42.1 \%$. Thus, about $84 \%$ are zoochorically dispersed in some way.

Three coevolutionary patterns may be distinguished between fruits and dispersal agents (as categorized above):

I. Large-seeded nutritious fruits seem to have coevolved with specialized frugivores as their principal dispersal agents. As McKey (1975) pointed out, the evolution of adaptations to specialized frugivory has resulted in generally increased dispersal quality, which means that the chance to survive and to grow to maturity per pro- 
pagule is greater than in both other seed dispersal strategies mentioned below. Specialists are more dependent on fruits as food, so one may expect that these animals possess a better ecological knowledge, especially of available fruit sources (as is demonstrated in Ateles, with their well-developed spatial and temporal memory for particularly nutritious fruit sources). The plant may xely on the regular visitation of specialists to the fruit-crop, and therefore the chance that mature fruits remain on the plant and become rotten, desiccated or predated after spontaneously falling to the forest floor is minimized. Furthermore, the quality of dispersal depends on the treatment of the seeds in the gut of the dispersal agent. Seeds adapted to dispersal by ingestion (endozoochory) have protection against the rough chemistry and/or physics experienced in the guts of animals. This defense may be a hard seed coat or endocaxp. Many specialists have thin-walled, often small stomachs with little muscularization, reflecting the relatively small amount of mechanical breakdown necessary for digestion of fruit flesh (Jenkins, 1969). Another adaptation in many specialists, especially in birds, is the habit of regurgitating larger seeds just after the surrounding edible part has been removed in the stomach (Skutch, 1944; Snow, 1961, 1962, 1970; McKey, 1975). Both these features mean that seeds ingested by specialized frugivores will receive a gentle treatment within the gut. Spider monkeys do not regurgitate seeds, but when eating large-seeded fruits they do not masticate much and seldom destroy seeds with the teeth. Theoretically, coevolution with specialists would involve the possibility of evolving relatively soft seeds which are not protected by a tough of endocarp. Indeed, this pattern of coevolution may be seen in many representatives of the family Lauraceae, which produce nutritious fruits containing one vulnerable seed that is protected from exploitation by generalists and seed-eaters by means of its size and by containing toxic compounds. This family figures strongly in the diets of a number of specialized frugivores (Snow, 1971). Howerer, it does not in the diet of Ateles in the Voltzberg area because most representatives of this family here measure less than $12 \mathrm{~m}$ in height. Finally, large seeds have a greater store of reserves than small seeds, and therefore the number of safe sites in a habitat is positively correlated with the increase in seed size. In some environments, selection favors an increase in seed size and a corresponding decrease in seed number 
(Janzen, 1969). If larger seeds are evolved, the plant has to produce a fruit nutritious enough to attract specialized frugivores capable of ingesting large seeds. The high nutritive content of these large-seeded fruits is notable, and this coevolutionary patterm may be demonstrated in families such as Burseraceae, Capparaceae, Loganiaceae, Meliaceae, Myristicaceae, Palmae, Sapindaceae and Sapotaceae, all of which produce important fruits for Ateles.About $80 \%$ of the 166 plant species producing edible fruits that are eaten by. Ateles p. paniscus, are of this nutritious and large-seeded type. In contrast, juicy, unnutritious and small-seeded fruits such as those of Bellucia, Campomanesia, Ficus, Guettarda, Hylocereus, Jacaratia, Passiflora, Pereskia and Trymatococcus only make up about 20\% of the total number of fruit species eaten by Ateles. Moreover, these fruits were usually exploited only incidentally on the way from one nutritious fruit source to another, and appear to never significantly influence the daily foraging route. Also, food sources of this kind were not regularly revisited (table IV).

II. Many animals feed primarily on insects and/or meat, and only utilize fruits as a source of water, organic acids and carbohydrates (Morton, 1973). Plants depending on generalists for their dispersal may be able to attract them with relatively inexpensive, low-or unnutritious fruits (e.g., berries). Generalists will choose to ingest a berry with small seeds rather than a protein-and fat rich fruit with a large seed, as long as proteins and lipids can be derived more easily from other resources (e.g., insects). Furthermore, most nutritious fruits are protected against exploitation by generalists by a strong attachment of edible layer to seed coat or endocarp and/or a toxic seed coat or endosperm. When the fruit is small, the outer layer is thin and leathery, facilitating swallowing of the whole fruit (e.g., many Melastomataceae, Myrtaceae, Rubiaceae, Cecropia, Coussapoa and Ficus). In larger fruits of this type, the outer layer is easily penetrable (e.g., Carica, Jacaratia, Hylocereus and Passiflora) or dehiscent (e.g., Carpotroche, Clusia, Phenakospermum and Stenosolen). The seed coat or endocarp is always very tough in fruits of this category, so the seeds are protected against the rough chemistry and mechanical breakdown in the gut of at least some of the numerous generalists that exploit them. Seeds of this kinds are mostly the so-called 'dormant' seeds, which can wait sometimes for years (or even decades, e.g., Carica) for a chance to germinate. Germi- 
nation in some species is induced by strong fluctuations in soil temperature (e.g., Cecropia, Boerboom and Holthuyzen, 1980), as may occur in edge habitats, seoundary formations and high forest following treefalls. Several plasits with fruits of this kind are true pioneers (e.g., Carica, Cecropia, Ficus, Miconia, Passiflora, Phenakospermum and Solanum. An advantage of this seed dispersal strategy may be the greater quantity of dispersal delivered by a wide anray of generalists and the longivety of seeds. However, this may come at the cost of decreased dispersal efficiency, since many seeds will be destroyed in the guts of certain animals. In addition, visitation of the fructifying plant is irregular and unreliable, the majority of seeds will fall to the ground and be predated under the parent plant, and the store of reserves is minimal. Furthermore, the range of habitats is restrictive.

III. The third zoochorical dispersal strategy found in Neotropical plants is that performed by scatter-hoarding rodents. This strategy is based on predator-satiation. The seeds are usually large and edible, and crops are mass-ripening. The seeds fall in quantity and are partly buried by scatter-hoanders effectively protecting them from predation by bruchid weevils. This coevolutionary pattern between fruits and dispersal agents is demonstrated in many Bombacaceae (e.g., Pachira), Lecythidaceae (e.g., Bertholletia, Eschweilera, Lecythis), Leguminosae (e.g., Andira, Vouacapoua), Meliaceae (e.g., Carapa) and Palmae (e.g., Astrocaryum, Attalea, Mauritia). The advantages of this strategy are the relatively low investment in edible layer, the great store of reserves in the seeds, the regularity and reliability of visitation by scatter-hoarders and the storage of seed in the proper habitat. Disadvantages may be the high pre-and post-dispersal predation loss, the high investment in nutritive seeds and the clumped appearance of seedlings and full-grown trees.

Since a specialized frugivore must extract a balanced diet from the fruits available at any one time, its existence may require overlapping fruiting seasons. This pattern may be shown in the case of the spider monkey. Most nutritious fruits eaten by Ateles p. paniscus have a definite fruiting season. Many of the fruiting seasons are quite long and overlap broadly. Most Burseraceae, for example, fructify for 2-3 1/2 months, Capparis maroniensis for 4 1/2 months, Dimorphandra pullei for 7 months, many Inga species for 2-3 
months, Strychnos tomentosa for 7 I/2 months, Guarea grandifolia for 4 months, Moutabea guianensis for 4 months, Euterpe species for two months, Oenocarpus bacaba for two months, Apeiba species for 2-3 months, Virola melinonii for 5 l/2 months and Licania majuscula for 5 months. In most nutritious-fruit producing plants exploited by spider monkeys, the length of the fruiting season is determined by the individual plants of the species not fruiting simultaneously and by the spread of truit maturing within one plant. The fruiting seasons were determined for 62 species producing nutritious fruit at the Voltzberg study site. Of these, 52 fruited for periods ranging from two to eight months. Spreading of fruit production over a long period of time. has the advantage that less strenuous demands are being made on the small community of dispersal agents. Plants following this strategy have their own reliable subset of dispersal agents, shared with relatively few other plants. In contrast, 14 of the 20 species producing low-or unnutritious fruit fruited for periods of less than two months (range 10-40 days). Since each species with a broad-niched fruit utilizes a large proportion of the available dispersal agents when it is fruiting, there will be strong selection for fruiting seasons of such species to be displaced in relation to one another, as has been clearly observed in Ficus. Altematively, the fruiting periods will be spread over extremely long periods of time, as has been observed in Cecropia and Bellucia. Plants dispersed by scatter-hoarders are usually mass-ripening, and at an individual level are producing crops only once every two or three years.

In summary, Ateles, as an important dispersal agent, shows many features of coevolution with nutritious, large-seeded fruits, and at least fills the niche of similarly adapted animals in the past. The high-quality dispersal offered by spider monkeys, which is offered by few other specialized frugivores, compensates the plant for the cost of producing crops ob nutritious fruits, but severely limits the number of propagules. In general, the fruiting seasons of these plant species are long, since the small number of dispersal agents may be easily overloaded by a mass-ripening fruit crop, and the existence of the dispersal agent may require that several species of fruit, each providing different nutrients, have broadly overlapping fruiting seasons. 


\section{SOCIAL ORGANIZATION}

\subsection{Introduction}

The social organization of spider monkeys is quite unsual among primates and thus far only chimpanzees have been found to exhibit a somewath similar system (Azuma and Toyoshima, 1962; Reynolds and Reynolds, 1965; Goodall, 1973; Sugiyama, 1973; Bygott, 1974; Wrangham, 1975). The similarities between spider monkey and chimpanzee organization is discussed in chapter 7 .

Spider monkeys live in social groups, networks or communities (Carpenter, 1935; Klein, 1972; Klein and Klein, 1975; Cant, 1977). Klein and Klein (1975) defined a social group of spider monkeys as 'a network of animals that usually interact peacefully with one another, while different groups are separated from one another by agonistic interactions'. For $A$. paniscus a more comprehensive definition is used. A social group of spider monkeys is here defined as an established number of animals, that all use the same area ('home range') and usually interact peacefully with one another, with only the adult males acting territorially and respecting clearcut boundaries. According to this definition groups are separated from one another by agonistic interactions conducted by the males, mainly by means of calling behavior and with considerable distance between opposing animals. Very occasionally, a sort of boundary conflict was observed. It was initiated by one or more males near the boundary and consisted of very agitated long calling and barking. This usually caused a rush of nearby subgroups towards the spot. During these rare incidents, its was not possible to observe individuals of both groups at the same time because of the distance between opponents, but probably visual contact also took place between the two groups high in the canopy or in emergents. Males of different groups were never observed attacking one another, but this may happen. Carpenter (1935) reports on few instances of fighting males, and collected several specimens showing large scars on hands, shoulders and head.

In $A$. paniscus, females usually stay within the boundaries of

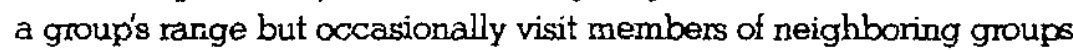
for periods ranging from several hours to about one full day, someti- 
mes even staying overnight with members of adjacent groups. These visits appeared to be undertaken particularly by females with newborn infants, and they seemed to involve showing the infant to the neighboring group. At these times, several members of the resident group were seen clustering around mother and infant, touching and sniffing the infant carefully. Agonistic behavior by any member of the resident group towards the visitors was never observed during these visits. Once, in the late aftemoon, I followed two females and a juvenile-3 male over more than one kilometer outside the home range, where they contacted some members of the adjacent group and stayed overnight with them. The journey was initiated by the leading fernale after she heard a male of the adjacent group repeatedly giving long calls far away. When crossing the group's range boundary, they left behind another female with a juvenile- 2 and a male, previously belonging to their subgroup. In this particular case no newborn infant was involved. This behavior may be explained as visiting one or more relatives since there are indications that emigration may take place, especially in the case of young females.

\subsection{Group Size, Group Composition and Age Classes}

One of the most striking characteristics of spider monkey social organization appears to be that all members of a given group can never be observed in the same place and at the same time. At the end of the field period, the study group consisted of 18 animals: thre adult males, eight adult females, one subadult female, four juveniles (one juvenile-3 male, one juvenile- 2 male, two juvenile- 2 females), and two infants (cone infant- 1 female, one infant- 2 male). The largest subgroups ever observed in the study group contained nine individuals, and consisted of either three males, four females and two juveniles, or of two males, four females and three juveniles. Two other groups that were regularly encountered in the Raleighvallen-Voltzberg Nature Reserve contained 15-20 individuals, of which three or four were adult males. The overall impression is that the study group can be regarded as an average group according to group size and group composition.

For $A$. paniscus, age classes are distinguished in the field and differentiated on the basis of size and dependence on the mothex. Compared with data given by Klein (1972) for $A$. belzebuth, by Eisenberg 
(1976) for $A$. fusciceps and $A$. geoffroyi, and by Cant (1978) for $A$. geoffroyi, dependence on the mother in infants and juveniles appears to be prolonged in feral $A$. paniscus.

Infants are clearly divided from juveniles by their invariably being carried by the mother during progression. Infant-1 (II) animals cling to the ventrum of the mother and range from zero to six months of age. Infant-2 (I2) animals ride on the back of the mother and range from six to 12 (or even 15) months of rage. In the intermediate phase, for about two weeks, infants were seen clinging at the lateral side of the mother during progression. Infant- 2 animals become more and more independent in locomoting, while the mother rests. They usually start to eat solid food but are still highly dependent on the mother for nourishment. The color of the coat in infant $A$. paniscus is the same as in adults, but naked face is paler. The skin around the eyes and on the muzzle is more darkly pigmented and gradually changes to light pink in juveniles or subadults and to pinkish or bright red in adults.

Juveniles usually locomote independently during progression, but stay permanently with the mother and are still nursed. Three size classes are distinguished. Juvenile-l (Jl) animals are 12 (or 15)-24 months of age. They are usually independently locomoting. However, when they seem to be fatigued (as when the group is traveling with increased speed) or during aggressive display of subgroup members they still may ride on the mother's back. Also, gaps between adjacent tree crowns are usually crossed with the help of the mother, who may bridge the gap with her body or leap across the gap with the young juvenile on her back.

Juvenile-2 (J2) animals are 24-36 months of age, and are able to keep up with a fast-moving subgroup. They were never observed riding on the mother's back and bridging was observed only very rarely. Eisenberg (1976) reports that in captive $A$. fusciceps and in free-ranging $A$. geoffroyi of this stage, juvenile males show an increasing affiliation with subadult and adult males. This pattem of affiliation was also observed in wild $A$. paniscus, but Klein (1972) did not observe it in feral. $A$. belzebuth. When meeting with other males, many times juvenile males (but never juvenile females) were observed mutually embracing, pectoral sniffing and swiffing each other's scrotum. Weaning usually takes place at the end of the juvenile-2 
stage, although suckling behavior was also observed in juvenile-3 males. Weaning in $A$. belzebuth is estimated to occur between two and three years of age (Klein, 1972), and in captive $A$. fusciceps Eisenberg (1976) observed intermittant suckling until 18 months of age.

Juvenile-3 (J3) animals are 36-50 months of age and stay with the mother most of the time. They play mainly with juveniles of about the same age or with aduits. Juvenile- 3 animals were ofter observed taking the initiative in leaving a food source and traveling in front of a subgroup in some parts of a route, that had been followed one or more times before. Juvenile-3 males start to share male longcalling and develop their own long call by trial and error.

Subadults are 50 to about 65 months of age. Female subadults were observed to stay almost permanently with the mother, even after the mother had given birth to an infant. Subadult males, however, were never observed to form a prolonged association with mother and infant, and they seemed to range independently from the mother. Subadults could be distinguished from adults by their face color and pigmentation and by their denser fur.

\subsection{Group Range and Day Range Length}

The annual home range of the spider monkey group in the Voltzberg study area covered 255 hectares, of which 220 hectares offered suitable habitat in the form of high forest, high mountain savanna forest and pina swamp forest. The perimeter of the group's range was determined for a greater part by natural boundaries, such as open granite llats, rocksavanna, low forest and liane forest. In areas where the range bordered the ranges of adjacent groups, it appeared to be clearly defined and non-overlapping.

Few estimates of home range size are available from other areas. Dare (1974) noted a range of 100-115 hectares for the $A$. geoffroyi group reintroduced on Barro Colorado Island. Klein and Klein (1976) estimated ranges of about 260-390 hectares with $20-30 \%$ overlap for $A$. belzebuth in La Macarena National Park in Colombia.

Travel distances trom day to day were highly variable for $A$. paniscus in the Voltzberg study area, depending on the subgroup size and composition, the weather and the season, and the distribution patterns of particular food plants. Straight-line distances bet- 
ween the consecutive food sources and sleeping trees used in one day could range from 500 meters (e.g., for a solitary ranging male or non-leading female during the long dry season) to 5,000 meters (e.g., for a subgroup led by a female during the long wet season). Klein (1972) gives minimal day ranges from 500 to 1,800 meters, and estimates the upper range of straight-line distances to be as large as 4,000 meters. For $A$. geoffroyi on Barro Colorado Island the upper range is given as 3,000 meters (Richard, 1970).

\subsection{Grouping Behavior}

Members of a spider monkey group associate in temporary subgroups or parties of changing composition and variable size. As a criterion for determining which individuals belong to a particular subgroup or party, mutually interdependent actions in feeding and traveling were used. For example, one female usually travelled about $50 \mathrm{~m}$ behind a subgroup that consisted of a minimum of one male, one female and one juvenile. She kept her distance because of agonistic interactions with the male (in particular when feeding on the same food source), but was nonetheless considered part of the subgroup. Also, when a party split but both subgroups obviously kept in visual and/or auditory contact while traveling from one food source to another, and following slightly different routes, all animals were considered to belong to one subgroup during that period.

As stated before, several females can easily be recongnized as leading a subgroup and determining daily itineraries and activity patterns of the subgroup as a whole. These females usually leave the sleeping tree first, travel in front of the subgroup most of the time, always feed on the food sources that determine the route that is taken, and initiate most activity patterns. They appear to possess a better knowledge of available food sources within the 'core areas" than other adults. To this end, they follow the constantly changing phenological picture by frequently monitoring different food plants to determine stage of flushing, flowering or fruiting. Regularly, in particular during the late afternoon, leading females were observed checking certain food sources in order to incorporate them into the itineraries of the days or weeks to come. While the female conducted these activities, the rest of the subgroup associated with this female could be observed traveling towards a well-known food source or sleeping tree nearby. When the leading female was accompa- 
nied by a juvenile, the offspring might follow her, or play in the meantime with another juvenile or adult, or remain with the traveling members of the subgroup. Figure 39 shows travel paths determined by a leading female, while she lead the frequently changing subgroup on consecutive days (i.e, on February 11, 12, 13 and on February 15, 16, 17, 18, 1978).

The route taken by a leading female seemed to be preplanned and highly economic, and used the shortest possible connections between consecutive food sources (as shown in figure 39). Doublingback rerely took place, and when it did, it seemed to be caused by confusion (e.g., after meeting and joining with anothe subgroup and following a route determined by the other leading female). By constrast, solitary or non-leading females seemed to be incapable of planning an economic route along a large number of food sources without making some loops and using certain important and well-known food sources repeatedly. Also, knowledge of available food sources and their exact locations appeared to be restricted among these animals, and resulted in a less varied diet (during the period in which they were on their own) that was mainly composed of foods from particular food sources wellknown to most or all members of the group. For males and nonleading females it would appear to be advantageous to regularly join subgroups led by a female in order to learm, through conspecific cueing (Kiester and Slatkin, 1974), about food sources avaible at the moment.

In total, four leading females could be recognized in the study group, and three of them were permanently accopanied by a juvenile at the end of the field period. One leading female did not have offspring. Most observations were of two leading females, one with a juvenile- 3 male and one with a juvenile- 2 female, that used partly 'cverlapping core areas with the camp situated about in the center during most of the year. The combined core area was situated roughly between B8 and B20 (fig. 40). In addition to the two leading females and their associated juveniles, two males and two nonleading females without offspring (one of them gave birth to an infant at the very end of the field period) were encountered most frequently in this area. 


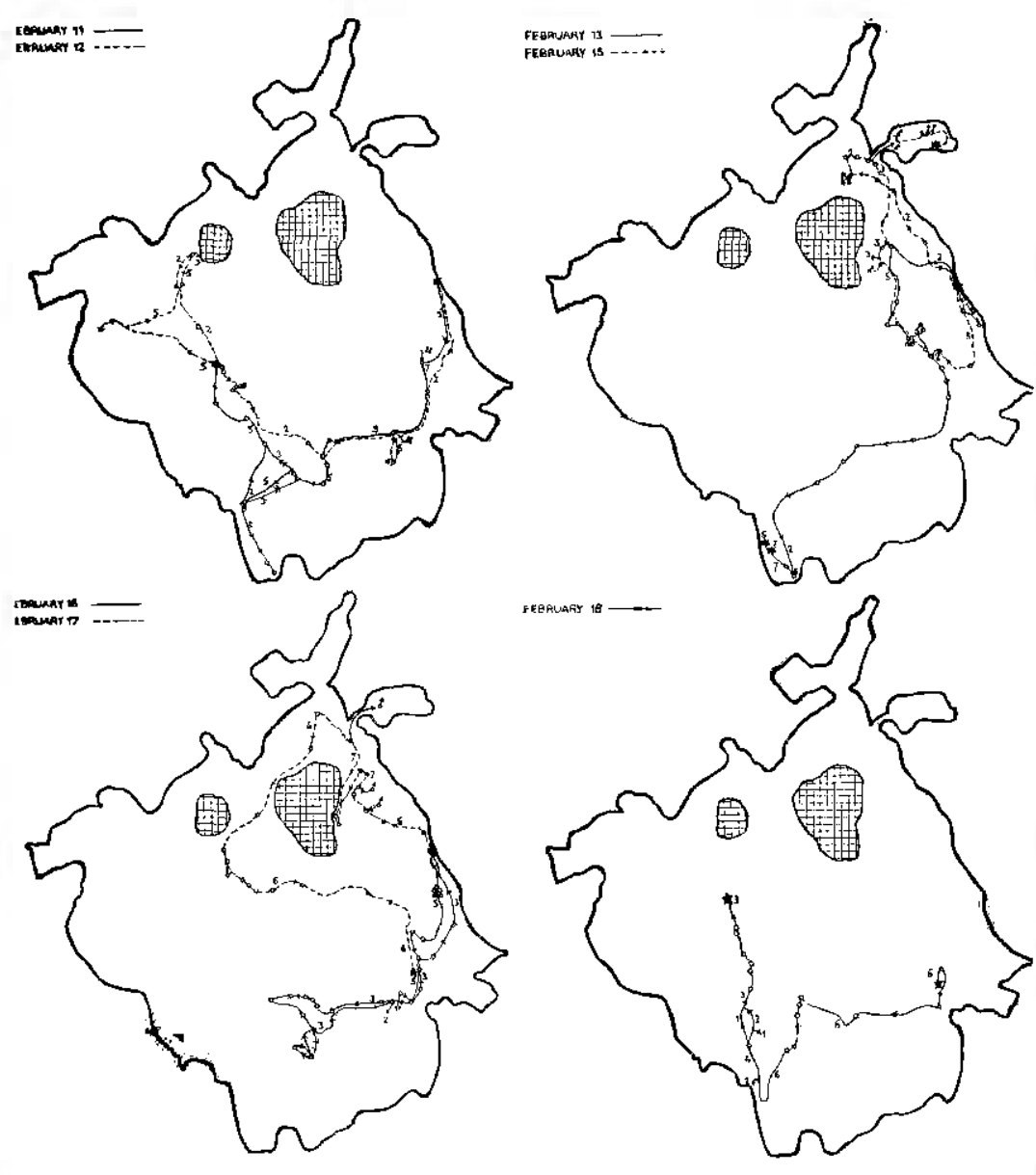

Figure 39 Daily travel paths and the pattern of temporary associations with the female (with juvenile) leading the frequently-changing subgroup on February 11, 12, 13, 15, 16, 17 and 18, 1978. Subgroup size, food plants and sleeping trees used are in. dicated. See for details figures $3 \mathrm{~g}, \mathrm{~A}, \mathrm{~B}, \mathrm{C}$, and $\mathrm{D}$.

In table XI, monthly association patterns between and with both leading females of the B8-B20 set are given by calculating the percentage of total observation time for four categories of subgroup composition: 1) no leading female(s) in the subgroup; 2) one leading female in the subgroup; 3) both leading females in the subgroup; and 4) one or more leading females in the subgroup. Observations were spread evenly over all six adults. For this purpose, a juvenile 


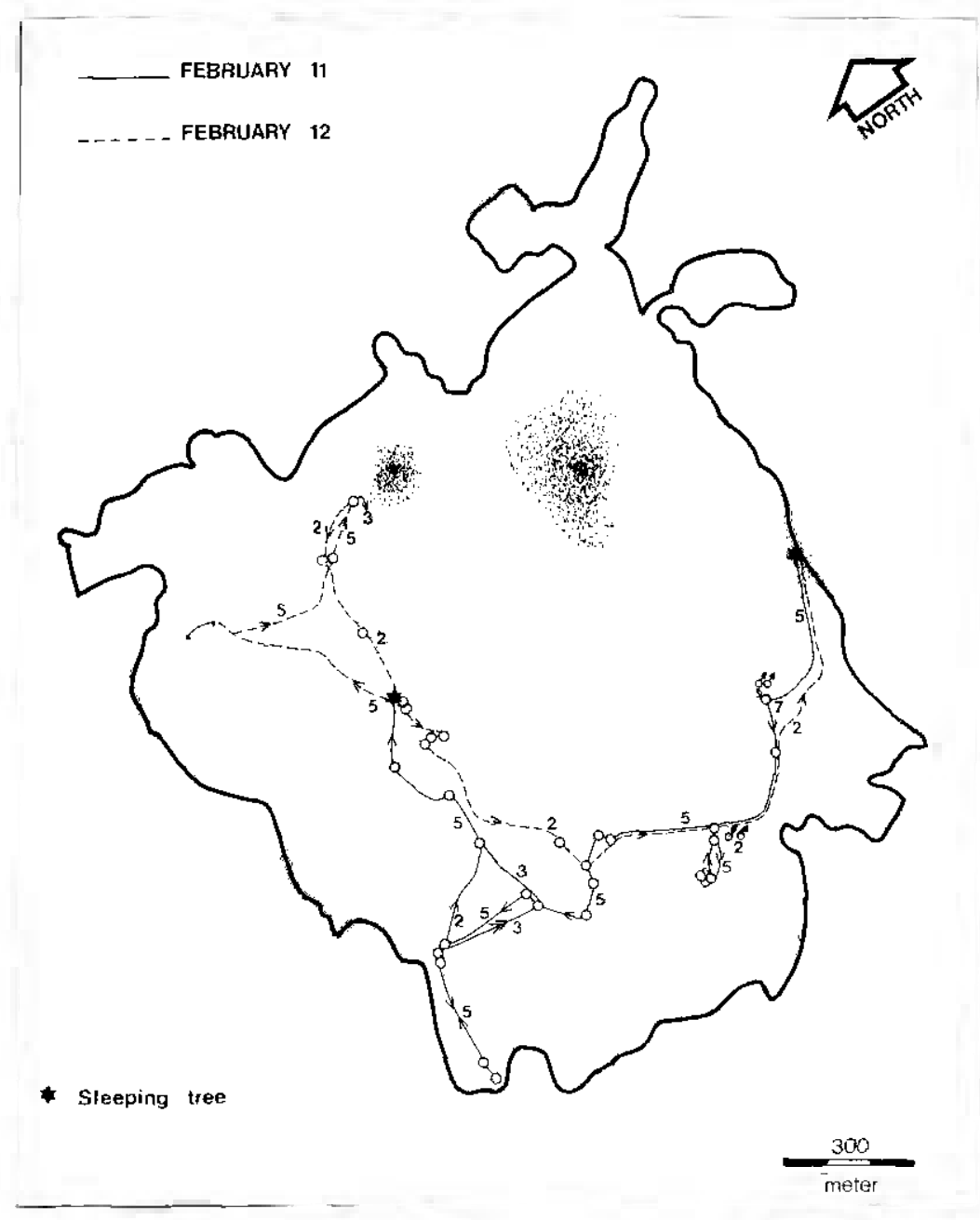

and its mother are considered a single unit. Thus, in these calculations six adults are involved: two leading females, two non-leading females and two males. The figures, given in table XI, clearly show a tendency for adults to assemble into subgroups containing at least one leading females thoughout the year, except for the months July and August ( 39.0 and $40.5 \%$, respectively) which represents a period of food scarcity (table X). The tendency is strongest from April through June (between 94.1 and $96.6 \%$ ), a period of food excess (table'X). 


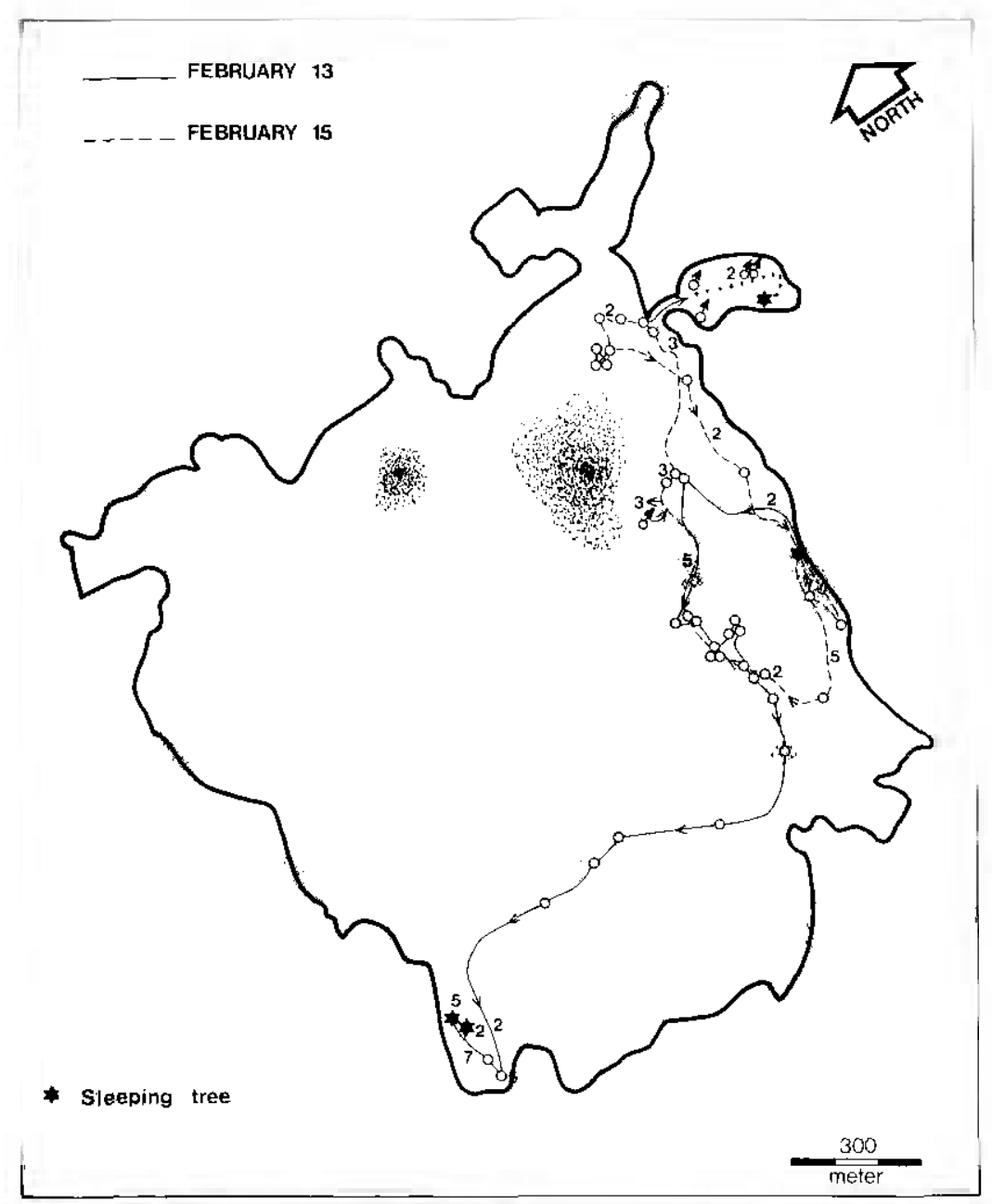

In table XII, all subgroup sizes and compositions observed in the study group during one year of intensive study are listed and their frequencies are expressed in terms of cumulative observation time (minutes) and percentage of total observation time (i.e., 1,061 hours). For this purpose, infants were considered together with their mothers as long as they were not fully independently locomoting during travel (i.e., from birth to about 12-15 months of age). Subgroups of three or less individuals were observed slightly more frequently than subgroups of four or more individuals (53.3 and $46.7 \%$, 


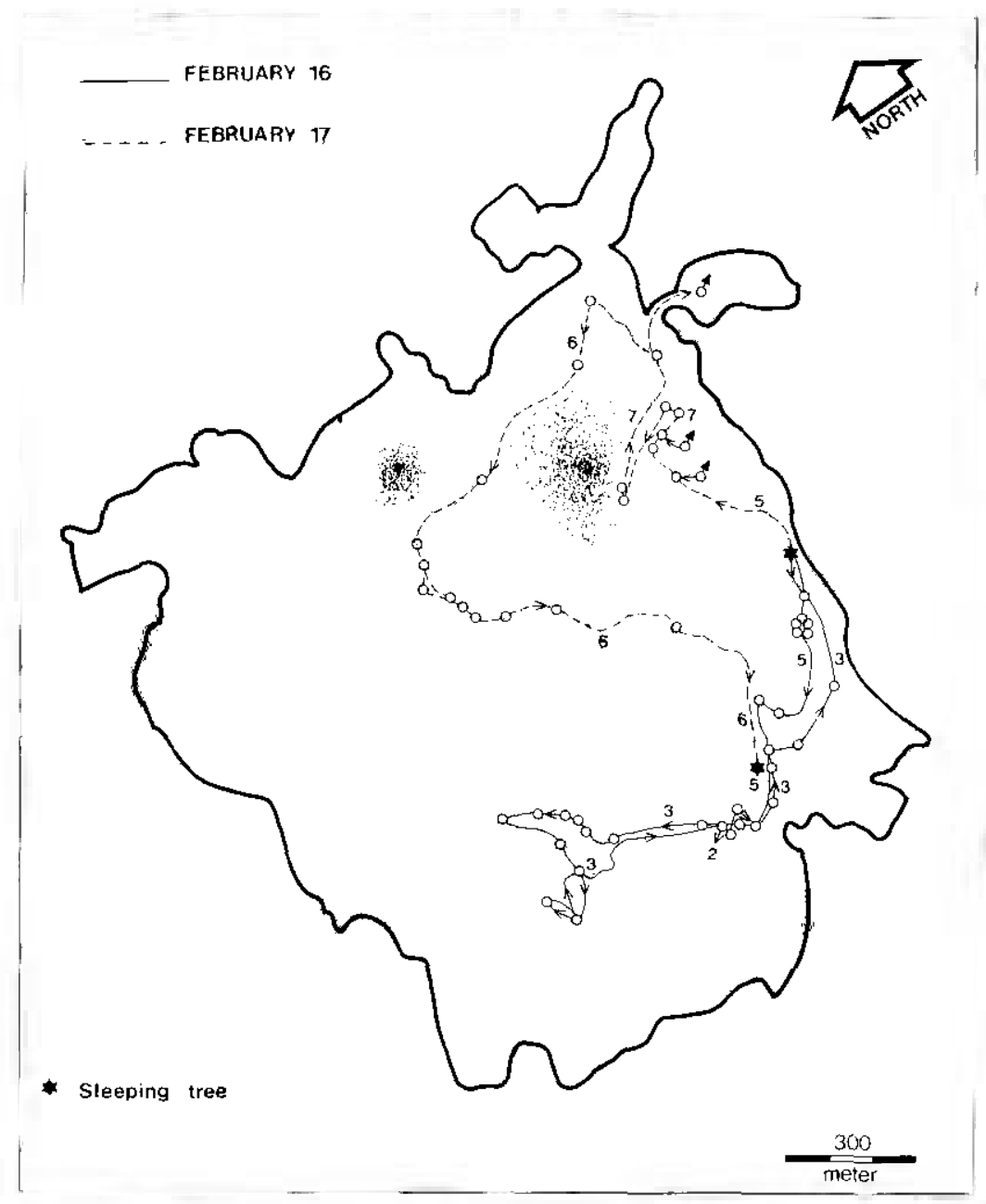

respectively). A subgroup of three individuals was observed most frequently $(28.0 \%)$, followed by subgroups of two and four individuals (19.1 and $18.6 \%$, respectively). Solitary ranging animals were seen with a frequency of $6.2 \%$, but they may be underestimated because of their being harder to detect. Subgroups of five, six and seven individuals made up for $12.7,9.0$ and $6.2 \%$, respectively, whereas subgroups of more than seven individuals only made up for $0.24 \%$ of total observation time. 


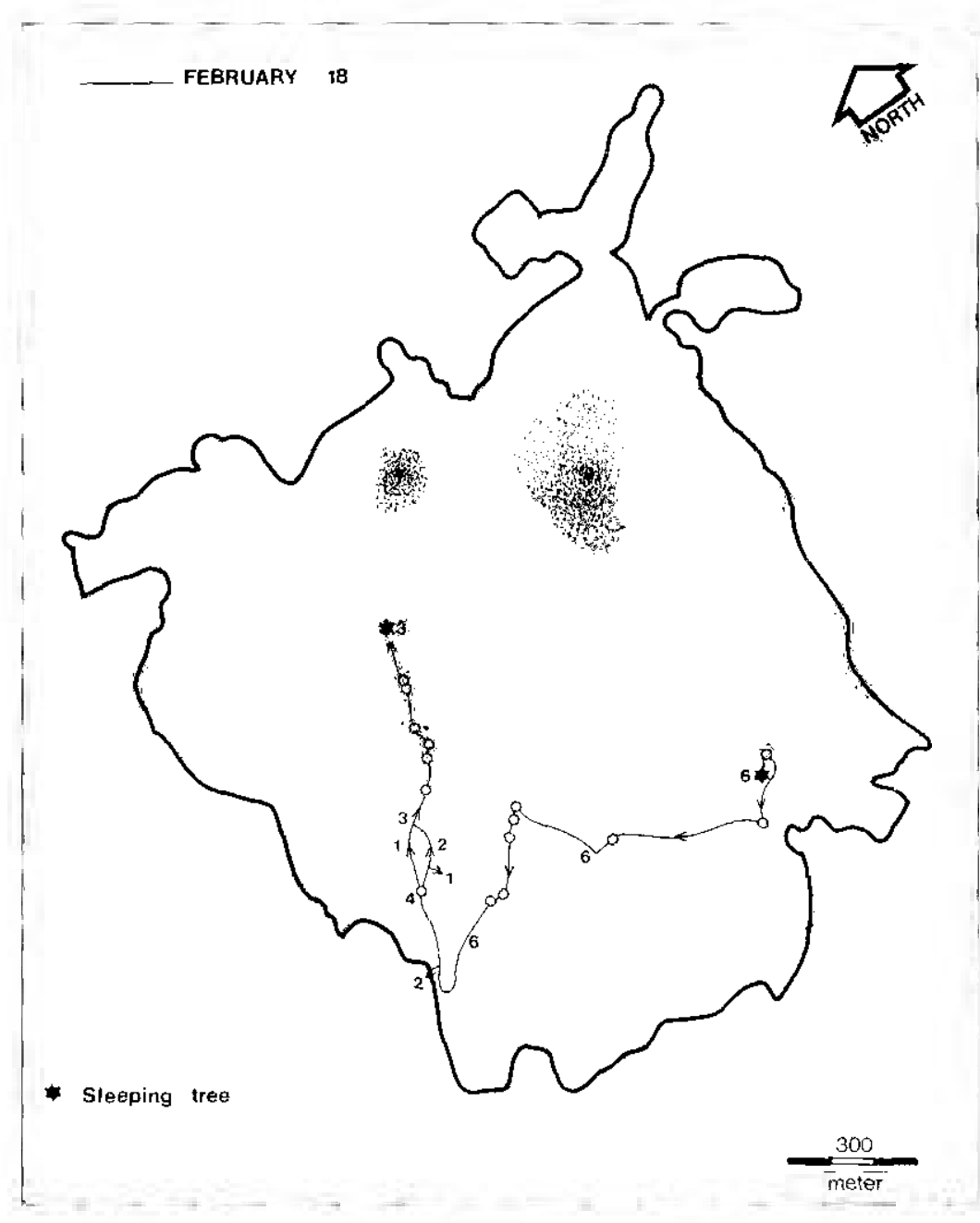

The subgroup composition most frequently observed was the combination $0^{\prime \prime} q+\mathrm{J}(18.5 \%)$, followed by that of $\delta q q+\mathrm{J}$ $(12.3 \%)$. The subgroup compositions $q+\mathrm{J}(8.9 \%)$ and $q$ \% $+\mathrm{J}$ $(8.6 \%)$ were also common. Solitary ranging males were observed with the same frequency (i.e. $3.1 \%$ ) as solitary ranging females with or without an infant but, as stated before, these frequencies may be regarded as underestimated. The combination male non-leading female was observed with a frequency 


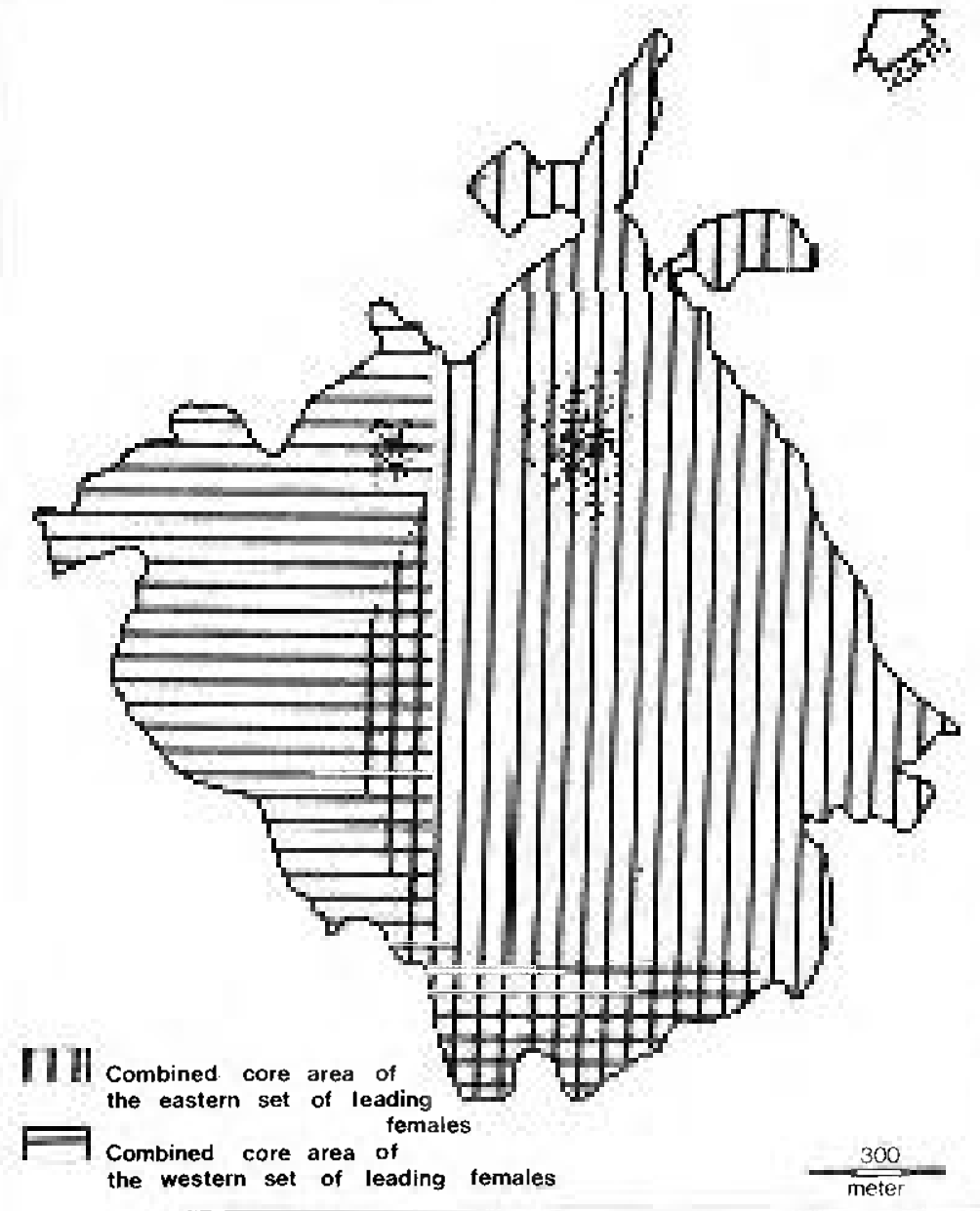

Figure 40 Distribution of combined core area of both sets of leading females within the spider monkey group range. The combined core area of the western set of leading ternales corresponds with the core area of male 32 , the combined core area of the eastern set of leading females corresponds roughly with that of male ofl and male 33 . Male 33 was tound most frequently in the core-area overlap zones where every group female could be encountered regularly.

of $6.1 \%$ and mainly involved a receptive female. A subgroup consisting of at least one male together with other animals was obser-" ved with a frequency of $70.9 \%$. A subgroup of only two males made 
Table XI. Monthly variation in frequencies of four different subgroup categories composed of individuals ranging mainly within the B8-B-20 area, the combined core area of one set of leading females, selected because it belonged to the best studied section on the spider monkey group. Six adults are involved: two leading females, two non-leading temales, and two males (male ...1 and ...3). For the sake of determining association partterns between and with leading females, juveniles were considered together with their mothers (both mothers were leading females). High monthly observation time figures from October through March are due to an assistant simultaneously gathering data on subgroups formed by fission from the focal subgroup.

\begin{tabular}{|c|c|c|c|c|c|}
\hline \multirow[t]{3}{*}{ Month } & \multicolumn{4}{|c|}{ Erequency of subgroups containing } & \multirow{3}{*}{$\begin{array}{l}\text { Total obeervatiox } \\
\text { time in minutes }\end{array}$} \\
\hline & $\mathrm{O}$ & One & Two & One or more & \\
\hline & leading & female(s) & & & \\
\hline January & 23.7 & 57.7 & 18.7 & 76.3 & 7,775 \\
\hline February & 27.1 & 39.6 & 33.3 & 72.9 & 9,562 \\
\hline March & 17.4 & 51.7 & 30.9 & 82.6 & 8,565 \\
\hline Aptal & 34 & 57.9 & 38.7 & 96.6 & 4.690 \\
\hline Mry & 2.2 & 67.5 & 30.3 & 97.8 & 3,000 \\
\hline Jung & 5.9 & 90.8 & 3.3 & 94.1 & 3,804 \\
\hline fully & 61.0 & 39.0 & 0.0 & 39.0 & 2,218 \\
\hline August & 59.5 & 40.5 & 0.0 & 40.5 & 2,288 \\
\hline September & 22.9 & 72.5 & 4.6 & 77.1 & 2,260 \\
\hline October & 19.7 & 62.0 & 18.3 & 80.3 & 8,018 \\
\hline November & 23.9 & 41.8 & 34.3 & 76.1 & 11,244 \\
\hline December & 9.1 & 72.5 & 18.4 & 90.9 & 6,750 \\
\hline
\end{tabular}

up for $3.9 \%$, whereas a subgroup of only three males was seen only in other groups in the Raleighvallen-Voltzberg Nature Reserve. Subgroups containing at least two males together with other animals were observed with a frequency of $15.7 \%$, whereas a large subgroup including all three males was observed in only $0.2 \%$ of total observation time.

Since spider monkeys are large animals that feed predominantly on mature, relatively nutritious truits not produced in large amounts simultaneously by a particular food plant, subgroup size has to be restricted to avoid competition in a given food plant. Theoretically, subgroup size in spider monkeys will be positively correlated with food patch size. In table XIII, all subgroup-size classes observed in the study group and the percentage of total observation time for each size class are given for each month of the year. To make the figures easier to interpret, subgroups-size classes have been reduced to two in table XIV: subgroup of more than three and subgroups of three or less fully independently locomoting individuals. Subgroups 
Table XII. Mean size frequencies of independent, aged and sexed subgroups during a full year observation. For this purpose, infants were considered together with their mothers, as long as they were not independently locomoting during travel. $\mathrm{I}=$ Lnfant, $\mathrm{J}=$ juvenile, $\mathrm{S}=$ subadult

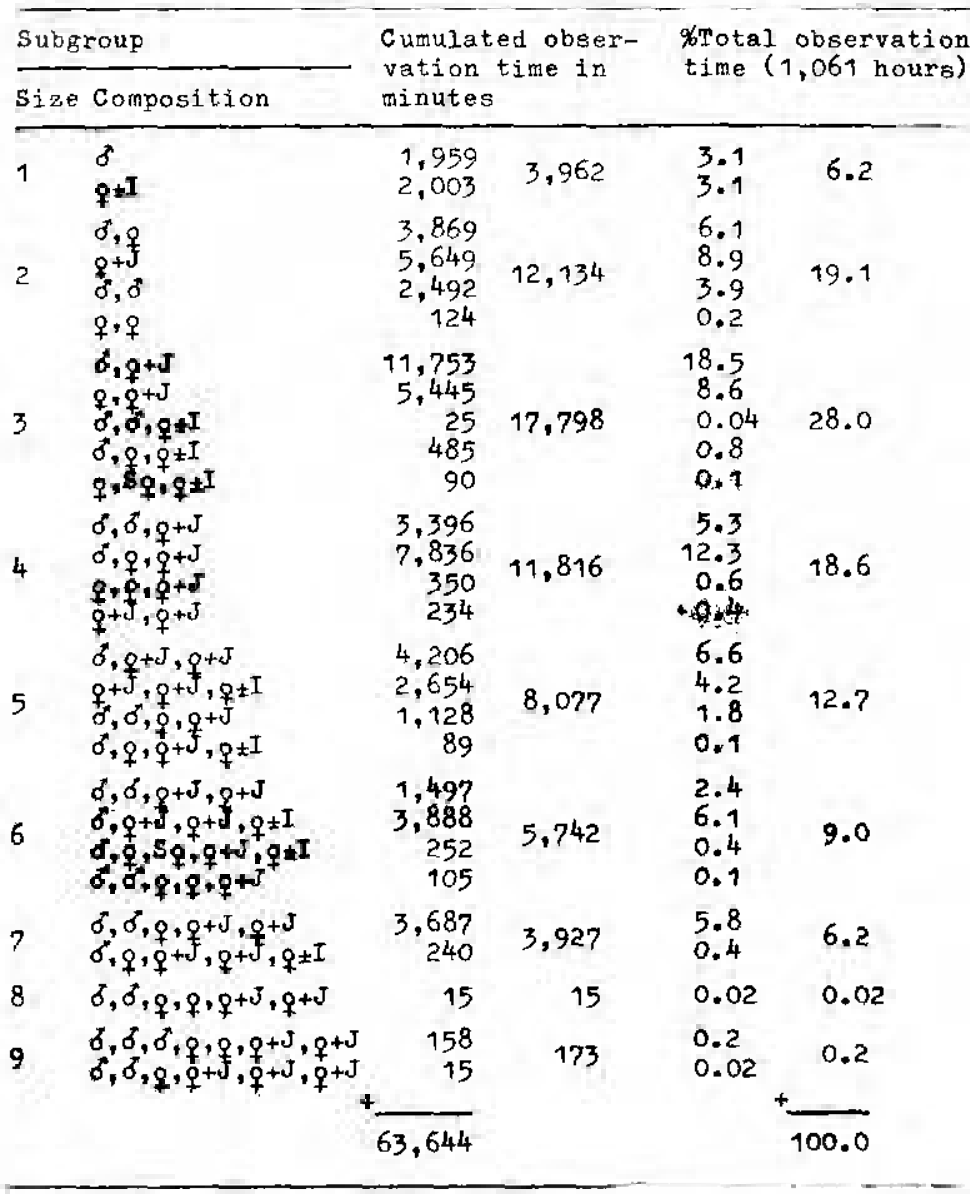

were smallest during the first part of the long dry season, a period of food scarcity (particularly of mature fruits), with significant minima in July, August and September $(26.8,18.4$ and $4.6 \%$, respectively, for subgroups of more than three individuals). In contrast, subgroups were largest during the peak of the long wet season when an excess of mature truits existed (table $\mathrm{X}$ ), with significant maxima in April and May (77.2 and 65.4\%, respectively, for subgroups 
of more than three individuals). During the rest of the year, figures are relatively constant and subgroups of more than three individuals range from 35 to $45 \%$.

In a subgroup of four or more independently locomoting animals following an itinerary determined by a leading female, rarely did more than three animals feed at the same time on one particular food source. In general, this only occurred when a sleeping tree also provided ample, food or when a large-crowned flowering or flushing tree offered enough space to feed at ease together. While the leading female with her offspring was feeding on a particular, usually primary, food source, one or more other animals (i.e., particularly non-leading females) could be seen searching for or feeding on nearby food sources, sometimes of less high quality. They often entered the food plant chosen by the leading female when the other animals were resting or moving to the next food source In this way, the itineraries followed are the same for all subgroup participants. Also, non-leading females and males can leam about important food sources and their location, information that they will need when they are solitary or associated with one or more non-leading female(s) and/or male(s). Clearly, this foraging pattern can be followed only when food supply is high and truiting food plants occur in relatively high densities, making it likely that another food source will be in the vicinity of the one chosen by the leading female. During the present study, this condition appeared to be fulfilled particularly in April, May and June, when trees of Guarea grandifolia, Proilum neglectum, Protium polybotryum, Tetragastris altissima and Tetragastris panamensis were fruiting abundantly. These species had overall densities of respectively $214,148,103,777$ and 133 fullgrown trees per square kilometer (table I) and locally reached much higher densities. 
Table XII. Monthly variation in size frequencies of independent subgroups, expressed as the percentage of total observation time per month for size classes of one to nine individuals. High monthly observation-time figures from October through March are due to an assistant simultaneously gathering data on subgroups formed by fission from the tocal subgroup.

\begin{tabular}{|c|c|c|c|c|c|c|c|c|c|c|}
\hline \multirow{2}{*}{ Month } & \multicolumn{9}{|c|}{ Number of individuals per subgroup } & \multirow{2}{*}{$\begin{array}{l}\text { Total } \\
\text { obs.time } \\
\text { in min. }\end{array}$} \\
\hline & 1 & 2 & 3 & 4 & 5 & 6 & $?$ & 8 & 9 & \\
\hline January & 19.4 & 8.8 & 36.9 & 15.2 & 4.2 & 14.5 & 0.9 & - & - & 7,869 \\
\hline February & 4.7 & 43.2 & 14.6 & 4.2 & 22.5 & 7.7 & 3.1 & - & - & 9,584 \\
\hline March & 17.1 & 3.0 & 37.8 & 11.0 & 7.9 & 13.0 & 10.0 & - & 0.2 & 8,578 \\
\hline April & 3.4 & 0.1 & 19.3 & 38.5 & 8.8 & 15.1 & 14.8 & - & - & 4.690 \\
\hline May & 1.0 & 21.4 & 12.2 & 46.2 & 8.2 & 3.5 & 7.5 & - & _ & 3,000 \\
\hline June & 5.2 & 29.5 & 19.8 & 41.6 & 3.1 & 0.8 & - & - & - & 3,834 \\
\hline July & 2.9 & 45.7 & 24.6 & 26.8 & - & - & - & - & - & 2,218 \\
\hline August & 10.5 & 24.1 & 47.0 & 18.4 & - & - & - & - & - & 2,288 \\
\hline September & 0.0 & 45.9 & 49.5 & - & 4.6 & - & - & - & _ & 2,260 \\
\hline October & 7.6 & 5.5 & 45.5 & 13.1 & 15.5 & 3.6 & 9.2 & - & - & 8,053 \\
\hline November & 7.3 & 21.9 & 24.6 & 10.2 & 15.9 & 9.6 & 9.0 & 0.1 & 1.4 & 11,619 \\
\hline December & 7.4 & 3.8 & 44.6 & 23.6 & 13.9 & 6.7 & - & - & - & 7,002 \\
\hline
\end{tabular}


Table XIV Monthly variation in size trequencies of two classes ( $\leqslant 3$ and $>3$ independently locomoting individuals) of indepentent suthotoups, expresses as the percentage of total observation time per month and the percentage of the total number of observed nightity subgroup sizes per month in the same size classes.

\begin{tabular}{|c|c|c|c|c|c|}
\hline \multirow[t]{2}{*}{ Month } & \multicolumn{2}{|c|}{$\begin{array}{l}\text { \%Total observation } \\
\text { time per subgroup- } \\
\text { size class }\end{array}$} & \multirow[t]{2}{*}{$\begin{array}{l}\text { Total ob- } \\
\text { servation } \\
\text { time in } \\
\text { minutes }\end{array}$} & \multicolumn{2}{|c|}{$\begin{array}{l}\text { Ootal night observations } \\
\text { per subgroup-size class }\end{array}$} \\
\hline & $\leqslant 3$ ind. & $>3$ ind. & & \& 3 ind. & $>3$ ind. \\
\hline $\begin{array}{l}\text { January } \\
\text { February } \\
\text { March } \\
\text { April } \\
\text { May } \\
\text { June } \\
\text { July } \\
\text { August } \\
\text { September } \\
\text { October } \\
\text { November } \\
\text { December }\end{array}$ & $\begin{array}{l}65.1 \\
62.5 \\
57.9 \\
22.8 \\
34.6 \\
54.5 \\
73.2 \\
81.6 \\
95.4 \\
58.6 \\
53.8 \\
55.8\end{array}$ & $\begin{array}{r}34.9 \\
37.5 \\
42.1 \\
77.2 \\
65.4 \\
45.5 \\
26.8 \\
18.4 \\
4.6 \\
41.4 \\
46.2 \\
44.2\end{array}$ & $\begin{array}{r}7,869 \\
9,584 \\
8,578 \\
4,690 \\
3,000 \\
3,834 \\
2,218 \\
2,288 \\
2,260 \\
8,053 \\
11,619 \\
7,002\end{array}$ & $\begin{array}{r}62.5 \\
60.0 \\
50.0 \\
25.0 \\
20.0 \\
37.5 \\
100.0 \\
100.0 \\
100.0 \\
60.0 \\
60.8 \\
77.0\end{array}$ & $\begin{array}{r}37.5 \\
40.0 \\
50.0 \\
75.0 \\
80.0 \\
62.5 \\
0.0 \\
0.0 \\
0.0 \\
40.0 \\
39.2 \\
23.0\end{array}$ \\
\hline
\end{tabular}


Two of the four leading females in the study group were intensively studied. Throughout the year, they used mainly the area between the parallels on B8 and B20. The other two leading females used the area between the parallels on $\mathrm{Bl}$ and $\mathrm{BlO}$, within the perimeter of the group's range (fig. 40). In addition to the strip between $\mathrm{B} 8$ and $\mathrm{B} 10$, an important area of overlap between the core areas of both sets of leading females could be found to the south of the B-axis, between B1O and B18. In this area, every member of the group could be encountered regularly. The non-overlaping areas were not exclusively used by the respective set of leading females with their associates, but seemed to be most familiar to them. The most intensively studied set of females using the area between $B 8$ and $\mathrm{B} 2 \mathrm{O}$, visited the second area (between $\mathrm{Bl}$ and $\mathrm{BlO}$ ) more frequently than the second set of females visited the first (i.e., the area between the parallels on $\mathrm{B} 1 \mathrm{O}$ and $\mathrm{B} 2 \mathrm{O}$, and to the north of A15). This was especially true during October and November when preferred food (i.e., mature, relatively nutritious fruits) was still scarce. The eastern subgroups were foraging regulary for infructescenses of Philodendron spp., fruits of Ecclinusa guianensis and flush leaves of Ceiba pentandra, foods which more often were present in the second area.

Leading females of each set usually exploited different but partly overlapping core areas within their general range. The core-area overlap appeared to grow with increasing food supply. In times of food scarcity (i.e., from July through September), one leading female of the eastem set almost exclusively exploited a core area situated between the B8 and B15 trails, and in particular to the south of the B-axis, whereas the other almost exclusively used a core area between the B15 and B2O trails, and in particular to the north of the A15 trail. Each core area covered about 100 ha. In times of food excess (i.e., during April and May) this set of leading females used almost all of both core areas combined, covering about 200 ha. The western set of leading females exploited a combined core area of about 120 ha.

Looking at subgroups containing both leading females of the eastern set together with one or more of the other four adults ranging mainly within this area, the following frequencies were observed: $18.7 \%$ (January), 33.3\% (February), 30.9\% (March), 38.7\% 
(April), 30.3\% (May), 3.3\% (June), 0.0\% (July), 0.0\% (August), 4.6\% (September), 18.3\% (October), 34.3\% (November) and 18.4\% (December) (table XI). During the first part of the long dry season (i.e., July - September), a period of food scarcity, both leading females almost never joined into one subgroup. In contrast, at the end of the long dry season, a peak of joining can be seen. One reason for this pattem of association may be the abundance of large crowned flushing and flowering tood plants (e.g., Ceiba pentandra, Vataireopsis speciosa) and several Bignoniaceae) in November. These offer enough food and space for relatively large subgroups to feed together (table XIV; $46.2 \%$, four subgroup sizes of more than three independently locomoting individuals). A second reason may be the already mentioned regular visits of both leading females, often together in one subgroup, to the core areas of the western set. Roughly similar frequencies of joining are reached from February through May when supply of mature fruits was high and several important food species fructified abundantly. An absolute peak of joining can be seen in April when an excess of mature fruit was most obvious (table X). Food supply in October, December and January was moderately high (consisting partly of flush leaves and flowers), resulting in medium frequencies of joining (i.e., about $18 \%$ ).

Considering the conspecific-cueing concept in foraging, as described above, the following question may arise: What mechanism governs the decrease of mean subgroup size in times of food scarcity, and when would it be most advantageous for males and nonleading females to leam about available food sources from leading females by joining their subgroups? Leading females of a set each seemed to prefer regular contact through merging of subgroups when food patch size and distribution allowed foraging in relatively large subgroups. The reason for this association pattern may be merely a social one. These females and particularly their offspring seemed to enjoy very much one another's company. When in the same subgroup, the juveniles spent much time playing together. The exchange of knowledge of available food scurces does not seem of importance for leading females. This may be demonstrated by the way both females of a set acted after merging subgroups. At these occasions, they alternately determined subgroup activity patterns and frequently separated into two subparties for short periods of time. Each female led part of the subgroup along slightly different 
itineraries before merging again. For the sake of foraging success, leading females obviously do not need one another. Therefore, no agonistic behavoir is needed to force them into exploiting different core areas in times of food scarcity. With this in mind, it may be interesting to note that already in June when food supply still was fairly high (table X), both leading females of the B8-B20 set assembled into one subgroup with a frequency of only $3.3 \%$ (table XI). This pattern may be explained by the following. For optimal exploitation of different, barely overlapping core areas in July, leading females may have to begin early to monitor future food sources in these areas. It may require some time to learn and memorize all food sources about to become available in a smaller but previously less frequently visited area. The above argued mechanism of leading-female core-area splitting alone already will drastically decrease mean subgroup size during the period of food scarcity. Males and non-leading females may join a leading-female subgroup or form small subgroups with one another. By calculating the monthly frequencies for each category of subgroup composition (table XI), it appears that subgroups of one or more individuals without a leading female were found much more frequently in July and August (61.0 and 59.5\%, respectively), than during the rest of the year. Whether this association pattern of males and non-leading females results after increased agonistic behavior from leading females is not fully clear. Regular overt aggression was observed by males towards non-leading females and juveniles. This occurred especially when they fed at the same time on one food source with restricted food supply. Some overt aggression was observed in July by a leading female towards the younger male within her subgroup. Aggressive behavior by a leading female toward a non-leading female was observed very rarely but was severe when it did occur. In this context, it may be adequate to note that a comparable type of appeasement behavior as performed mutually by males when meeting one another after some time, occasionally was observed in females. It was initiated by the non-leading female when meeting a leading female, and was attended with pectoral sniffing and mutual embracing.

\subsection{Male Behavior}

The study group included three adult males, an old male ( $\left.\sigma^{*} 1\right)$, one of middle age $\left(\delta^{3} 2\right)$ and a young male 6-7 years of age ( $\left.0^{3} 3\right)$. 
When meeting one another after some time, male spider monkeys belonging to the same group perform a kind of greeting ceremony composed of mutual embracing and pectoral sniffing usually followed by mutual sniffing of the scrotum. Greeting behavior among males invariably appeared to be initiated by the lower-ranking male, and it may be regarded as appeasement behavoir. The old male $(\delta 1)$ was the highest ranking, followed by male $\delta 2$, male $\delta 3$ and a juvenile-3. It seems that dominance relationshps among spider monkey males are largely determined by age.

Adult males were never observed outside the perimeter of the group's range, and usually (but not exclusively) ranged within certain core areas. The older males, $0^{x} l$ and $\delta 2$, each used approximately the combined core area of a set of leading females, the eastern and western set, respectively. The young male (83) alternately used both male core-areas but more often could be seen within the eas. tem area, and particulary within the overlap zones (i.e., the areas to the south of the B-axis and beiween the B8 and B1O trails) (fig. 40).

Each adult male appeared to prefer joining a subgroup of one or more group members ( $70.9 \%$ of total observation time), especially when it contained one or more leading females (60.0\%). The males usually associated with each female of a set, or more specifically with a certain leading female. For example, male 82, was observed most frequently in a subgroup led by the mother of the juvenile-3 male, whereas male $\$ 3$ altemately joined a subgroup led by a female of the eastern set or, less often of the western set.

Both male 82 and male $\$ 3$ were seen visiting the male 82 core area more often than the reverse, and such visits took place especially in the second part of the long dry season. In this period, the three males repeatedly came together with other group members in an emergent tree of the species Hymenaea courbaril (fig. 41, no. 38), which apparently functioned as a meeting place. On these occasions, the largest subgroups were observed and ranged from seven to nine independently locomoting individuals. The adults rested in the huge crown for hours, whereas the juveniles played with one another and/or with adults. Otherwise, the three adult males of the study group were observed together only during the very rare teritorial boundary conflicts with members of the adjacent group to the south. These conflicts started with one adult male becoming agita- 
ted and starting to perform long calls. In cooperation with one or more females, he performed siamang-like barking duets. These caused an invasion of subgroups from the vicinity, whereas the members of the other group responded with similar behavior at the other side of the boundary. High up in the canopy or in emergents, the males of both groups were probably in sight of one another at a distance of about 100-200 m, acted very upset and performed an aggressive display for a considerable time. The males shook branches and broke off twigs, branches and even dead boughs, and uttered long calls ("whoops") and barks in cooperation with one or more females ("ook-barking"; terms derived from Klein, 1972). Several times, the males were observed performing reassurance behavior by mutually embracing and pectoral sniffing or by briefly touching one another's back, shoulder or arm with the hand. These territorial conflicts, always initiated by a male, were very rare and were heard among other groups in the Raleighvallen-Voltzberg Nature Reserve only a few times during this study. The rarity of territorial conflicts between groups may be explained by the relatively few high forest boundaries with adjacent groups. Most of the study group's range boundaries were formed by geographical barriers, such as liane forest and open granite areas.

\subsection{Calling Behavior}

Only male spider monkeys perform long calls or "whoops". On the forest floor, these long calls are audible at a distance of $800-1,000 \mathrm{~m}$. High up in emergent trees, long calls can be heard over approximately $2,000 \mathrm{~m}$, whereas from the top of the Voltzberg $(240$ $\mathrm{m})$ calling males can be located at distances of 3,000-4,000 m. Assuming that the spider-monkey sense of hearing is at least as good as that of humans, spider monkeys in an emergent crown should be able to hear every intragroup long call. The longest distance between two points within the perimeter of the home range amounts to $2,400 \mathrm{~m}$.

Long calls by the different males of the study group could easily be recognized individually by their sound frequencies, and both the number and length of intervals between notes.

At least five types of male long call are distinguished, sounding similar superficially but performed in different behavioral contexts: 
1. Morning Long Call (MLC): Performed usually once or only a few times during the period of increased foraging activity (i.e., between $6.00-7.30 \mathrm{~h}$ ), and as a rule answered by one or more males of the same group. It seemed intended to inform males (and other. group members) of one another's location and direction of travel. Estrous females were also attracted using this call.

2. Evening Long Call (ELC): Performed usually only once, just before or after the male entered a sleeping tree. It seemed intended to inform all group members of the location of the sleeping site selected for the night. Nearby parties could therefore be attracted to join and form larger units. When more males were associated in a subgroup, usually only one of them gave the ELC. An ELC usually was not answered inmediately by other males. The males seemed to perform ELC's at different times, since they usually did not simultaneously settle for the night. In addition, ELC's were not given every day. For example, the males kept silent when it was raining. Also, ELC's were heard less often during the long dry season.

3. Food Long Call (FLC): Food long calls could be given throughout the day, in particular just before or during feeding on an important, usually primary food source that probably was wellknown to most os all members of the group. In most cases, FLC's were not answered. They seemed to be used for spacing purposes. The performing male often was part of a relatively large subgroup and merging of subgroups usually did not take place after a FLC. These calls seemed intended to inform other monkeys that a nearby food source was being depleted at that moment, and therefore might better be dropped from a leading-female's foraging route.

4. Contact Long Call (CLC): Contact long calls could be given by a male just after leaving or loosing contact with a subgroup, perhaps in an attempt to influence the direction of travel chosen by the female leading the subgroup. Sometimes the male attracted the subgroup and joined it again, but usually he seemed not to succeed. This behavoir could be observed, for example, when a male left a subgroup in order to contact another one nearby, but after meeting the second group decided not to join it. It could also occur when the subgroup led by a female took one of two possible routes. In that case, the male could stay for a while at the junction of both potential routes, giving CLC's repeatedly. 
5. Alarm Long Call (ALC): Alarm long calls could be given all day by males, especially when upset by the presence of a large carnivore (e.g., a jaguar) or an unfamiliar human intruder, by alarmed terrestrial animals on flight (e.g., tinamous, quails, irumpeters, curassows, agoutis, deer, peccaries), or by combinations of these factors. Also, they could be performed during long-distance agonistic behavior towards one or more adult males of a neighboring group. ALC's were combined with "ook-barking", usually performed in cooperation with one or more adults, and sometimes lasted for more than an hour. After calling, most aduits remained very unstable for a while and the slightest incident could cause repetition of the behavior. For example, the observer could cause the aggressive display to be directed towards him by simply stepping on a dead branch, even if he did not play a role in initjating it. ALC's seemed intended to attract nearby subgroups to share in an aggressive display, and were never answered by calls from other subgroups belonging to the same group.

During the first part of the long dry season, long calls were heard only occasionally. Spacing may be optimal when males are relatively silent, especially considering the small day ranges and the drastically decreased energy budget in this period of food scarcity. As it was for the observer, it probably was also much more difficult for individual spider monkeys to encounter subgroups at any given time. The aggregating component of male long-calling behavior is therefore of mimimal importance in this season. It seems to be more important during more favorable periods when day ranges are much larger and the energy budget is increased considerably. Moreover, during the long dry season the available food sources are usually quite different from those offered during the rest of the year, and are of the kind that make quick depletion unlikely. Ripe fruits of Apeiba spp. and Dimorphandra pullei are important food sources that are present for long periods, whereas foods such as flush leaves and flowers are usually widely dispersed and available in large quantities for a short period (table I.). During the months of July and August, males and non-leading females in particular could be seen remaining for an entire day or more within a small area that offered only a tew of these food sources, and much of this time was spent resting. The decreased energy budget may be explained not only 
by the decline of overall food supply but also by the dietary shift, with flush leaves and flowers constituting a considerable part of the diet. The proportion of time spent on feeding and moving is negatively correlated to the proportion of foliage in the diet (Clutton-Brock, 1977).

\subsection{Sleeping Behavior}

The tendency to aggregate in sleeping trees is illustrated in table XIV. The percentage of nights spent together is given for two subgroup-size classes consisting of ... 3and ... 3independently locomoting individuals. Comparing these percentages to day figures, a strong tendency to aggregate in sleeping trees can be noted only in May and June. A strong tendency for spacing is demonstrated in July, August and December when food supply is more limited (table X).

A spider monkey group uses miany sleeping trees during the year but only a few are important throughout the year. Few trees have the permanently open crown preferred in a sleeping tree (e.g., Hymenolobium spp., Parkia pendula and Vochysia tomentosa). Strategic location within the perimeter of the home range or core area may also play a role and the size of the food supply to be found in the vicinity of the sleeping tree during most of the year is also important. In total, 43 different sleeping trees were used by membres of the study group (fig. 41). The following species contributed the greatest number of sleeping trees: Buchenavia capitata (ten trees), (Vataireopsis speciosa (five trees), Couratari stelata, Hymenolobium petraeum and Hymenolobium flavum (four trees each), and Parkia pendula (three trees). Ranked according to the number of nights spent in trees, the following species were most important: Hymenolobium flavum (31), Vochysia tomentosa (16), Newtonia suaveolens (12), Buchenavia capitata (ten), and Vataireopsis speciosa (eight).

Spider monkeys seemed to use the following criteria in selecting a sleeping tree:

1. The tree has to be an emergent, not only determined as such by its absolute height but also by its relationship with the environment. The crown has to be completely free from the canopy beneath and is entered by swinging or leaping across a gap from the periphery of an adjacent crown or by ascending the huge trunk using 


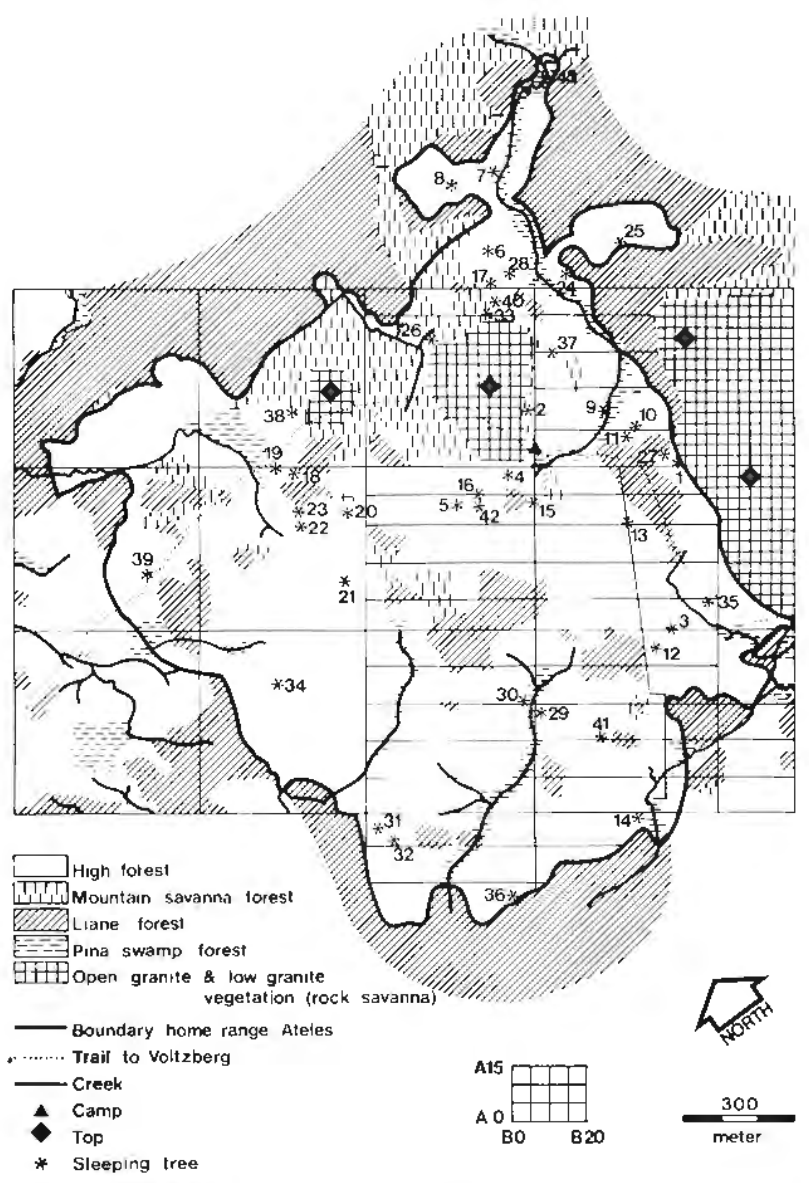

Figure 41 Location and species of sleeping trees used by members of the spider monkey group in the Voltzberg study area:

1. Hymenolobium flavum 2. Hymenolobium petraeum - 3. Enterolobium schomburgkii - 4. Couratari stellata - 5. Buchenavia capitata - 6. Vochysia tomentosa 7. Buchenavia capitata 8. Parkia pendula - 9. Dimorphandra multiflora - 10. Buchenavia capitata - 11. Cedrelinga catenaeformis - 12. Newtonia suaveolens - 13. Hymenolobium petraeum - 14. Parkia pendula - 15. Buchenavia capitata - 16. Vataireopsis speciosa - 17. Hymenolobium flavum - 18. Ceiba pentandra - 19. Parkia nitida - 20. Buchenavia capitata - 21. Buchenavia capitata - 22. Buchenavia capitata - 23. Buchenavia capitata - 24. Couratari stellata - 25. Parkia pendula - 26. Couratari guianensis - 27. Vataireopsis speciosa - 28. Vataireopsis speciosa - 29. Couratari stellata - 30. Vochysia tomentosa - 31. Vataireopsis speciosa - 32. Vataireopsis speciosa - 33. Hymenaea courbaril - 34. Hymenolobium petraeum - 35. Hymenolobium petraeum - 36. Hymenolobium flavum - 37. Hymenolobium flavum - 38. Hymenaea courbaril - 39. Buchenavia capitata - 40. Dimorphandra multifora - 41. Buchenavia capitata 42. Couratari stellata - 43. Qualea dinizii. 
stems of lianes, epiphytes and/or aerial roots. In particular, emergents at edges of natural clearings, granite outcroppings, low forest or liane forest are preferred for sleeping.

2. A broad, open crown that has small leaves, is currently leafess or is in early flush, is preferred.

3. The crown has to be structured with more or less horizontal branching. Spider monkeys seem to prefer almost horizontally forked branches for long resting postures, usually sleeping stretched out in a reclining posture or in a more or less asymmetrical sitting posture.

4. The tree has to be located strategically in respect to the foraging grounds of the late afternoon and the morning of the next day. Spider monkeys seem to be very hungry after awakening and prefer to have one or more important food sources situated near the sleeping tree, providing a good breakfast at the start of a day's itinerary.

5. A particular sleeping tree that provides the monkeys with a source of food may be given priority. This may be in the form of fruits, flowers or flush leaves of the sleeping tree itself, or edible epiphytes and/or lianes growing in its crown. When edible flowers or flush leaves are on the tree the monkeys prefer to consume these during the late afternoon, just before choosing their sleeping branch for the night.

Predation at night seems to be the only reasonable explanation for the strong selectivity in choice of sleeping trees fulfilling the first three criteria mentioned above. Another indication may be the fact that spider monkeys acted very upset and issued trilling squeaks when they were encountered in a sleeping tree late at night, probably being unable to identify the intruder on the forest floor. Occasionally, these specific vocalizations were heard at night near the camp, perhaps as a reaction to disturbance by a terrestrial carnivore or herbivore near the sleeping tree. All subgroup members, except infants, were scattered over a sleeping tree crown with preference for the center and the upper part, and always on branches. Each monkey appeared to possess its own sleeping 'branch ${ }_{r}$ at least in frequently used sleeping trees. By using slender perches in open and isolated crowns, the monkeys would be readily awakened by 
a felid or other predator climbing the tree. During the present field study, predation of spider monkeys was never observed. However, a young infant in the study group died for an unknown reason. Daytime predation probably does not present a problem for spider monkeys. Several times, spider monkeys were feeding in the periphery of open crowns with a harpy eagle (Harpia harpyja) flying in the vicinity, but in no way did they seem to be restrained by the bird's presence. Juveniles kept on feeding in vulnerable sites, and only once was a short branch-shaking display performed to scare off this large raptor. Some other observations may be mentioned in this context. Juvenile spider monkeys were observed curiously following tayras (Eira barbara) at very short distances for brief periods. Many hairballs and dung samples from the jaguar (Panthera onca) living in the area were found on trails and bare granite and were analyzed (Van Roosmalen, in prep.). They appeared to contain only nails, hair and bones of terrestrial animals. It is interesting to note that spider monkeys appeared to become particularly upset by alarmed terrestrial animals on flight, such as tinamous, quails, trumpeters, curassows, agoutis, deer, peccaries and tapirs. Ocelots (Felis pardalis) were living in the area, and once an ocelot was fighting with two tayras on the forest floor. A male spider monkey descended to about $10 \mathrm{~m}$ above the fighting animals and barked at them. An ocelot might be able to catch a juvenile spider monkey at night and even if this occurred only once per spider monkey generation, strong selective pressure for use of safe sleeping trees probably would be maintained.

Spider monkeys are very courageous animals, apparently not afraid of any day-time intruder, including man (unless they have prior experience with guns). On first contact with human intruders, spider monkeys usually perform a branch-shaking and branch-breaking aggressive display directed towards the intruder. Apart from this, the males may give long calls or perform "ook-barking" in cooperation with one or more adult females. Other subgroups may join the aggressive display. Dead tranches and even boughs, preferably those right above the intruder's head, are selected and broken off. This display may also be performed when detecting a large felid walking on the forest floor. 


\subsection{Reproductive Biology and Sexual Behavior}

According to Eisenberg (1976), spider monkey females giving birth to an infant will pass into a lactation anestrus for a period of 15.3-24 months. The data gathered in the Voltzberg study area, however, indicate a lactation anestrus for a period of about three years. An infant born in October 1974 was regularly nursed until September 19, 1977. A month later, its mother came into estrus for the first time. Another female gave birth to an infant in January 1975, and nursed it until December 1977. This female came into estrus again on January 22, 1978. Several females of $A$. paniscus in Surinam were nursing a juvenile- 2 or even a juvenile-3. Old juveniles were nursed only few times a day (mean duration of two minutes), usually while resting in a sleeping tree before nightfall. These few nursing bouts seemed to be sufficient to keep the mother in anestrus. The difference between the data from Eisenberg (1976) and the data collected on feral spider monkeys in Surinam may be explained by species specific differences or by the fact that social weaning for young spider monkeys in captivity is much earlier than in the wild.

Female spider monkeys coming into estrus show a tendency to manipulate, inspect and rub the clitoris. The duration of sexual receptivity in Surinam spider monkeys ranged from 8-10 days. The interestrus interval lasted an average of 15 to 17 days. This agrees with the cyclical uterine bleeding of adult female spider monkeys described by Goodman and Wislocki (1935) as occurring every 26 to 27 days.

In captivity, spidex monkey gestation has been calculated at 226 to 232 days (Eisenberg, 1973). The interbirth interval is a function of the duration of lactation and the number of estrus periods needed for successful conception. Interbirth intervals in captive $A$. fusciceps ranged from 22.8-31.5 months (Eisenberg, 1976). The range for $A$. geoffroyi is 17 to 45 months (Dare, 1974), 22 to 45 months (Eisenberg, 1976), or 28-36 months (Milton, 1981). Interbirth interval in feral $A$. paniscus in Surinam appeared to be four years (range: 46-50 months). These comparisons include only cases in which the mother reared her young to social weaning.

In $A$. paniscus, one of the leading females was seen copulating at least 11 times, spread over eight successive estrus periods. 
Another leading female was seen copulating at least seven times spread over three estrus periods. Successive estrus periods of five females belonging to the study group given in figure 42 . Unfortunately, at the end of the field period, the two most intensively-studied females had not yet become pregnant. Another female was lost after five successive estrus periods. The tifth female belonging to the western set of leading females copulated at least once with both male $\delta 1$ and male $\delta 3$ but regular contact was not maintained later in the study.

Klein (1971) suggests that captive $A$. geoffroyi and feral $A$. belzebuth in Colombia do not exhibit a discrete birth season. In contrast, Eisenberg (1976) indicates that $A$. fusciceps in captivity shows a tendency towards two birth peaks and suggests estrus synchrony. In addition, Milton (1981) presents evidence for seasonality in the distribution of births of $A$. geoffroyi on Barro Colorado Island. In Surinam, A. paniscus appeared to show a birth peak in the period November-February, from the end of the long dry season through the short wet season. None of six females shot by hunters in Lely Mountains in the period February-April 1976 contained a fetus (Mittermeier, 1977). Figure 42 shows estrus synchrony in four out of five $A$. paniscus females in Surinam in the period October 15 to at least April 26. The female giving birth to an infant on April 17, 1978 lost her previous infant in 1977, a fact that may explain asynchrony in estrus compared with the other females.

An estrus season from the end of the long dry season onto halfway the long wet season appears to agree with the seasonal variations in food patch size and grouping behavior as described earlier in this chapter. During the period of food scarcity, it should be unfavorable for females to come into estrus because of reduced frequency of encountering males and high energy demands of early pregnancy.

Males appeared to maintain consortship with receptive females usually not for the entire estrus period but at least for periods of time ranging from one to three days and nights. Also, consortship between two males and a receptive female was observed in A. paniscus and lasted as long as three days and nights. Female advertisement was observed prior to all copulations $(\mathrm{N}=27)$ and involved both leading and non-leading females. The receptive female always 
initiated sexual activity by moving rapidly towards a particular male, presenting her genitalia, and then sitting in his lap. When a male didn't react by initiating the copulatory position, the female rushed away through the tree crovin, sat down somewhere else, and after several minutes attempted a second or third time before the male locked his hindlegs around her torso. Consortship involving one or two males and a receptive female usually was not secluded from the other members of the group, particularly not in case of leading females. However, for copulating the pair sometimes sought seclusion. The reason for this is probably strong harrassment from the juvenile. Several times, a receptive mother of a juvenile separated from a subgroup just after entering a food tree when the juvenile paid full attention to foraging. In these cases, the male immediately followed the female, and the pair copulated undisturbed 100-200 na away from the rest of the subgroup.

Receptive females appeared to choose a mate. This was best demonstrated when a receptive female that ranged in a subgroup without males reacted to male long calls by immediately altering her direction of travel and rushing towards the calling male. After meeting the male, presentation and copulation followed. Once, a leading female responding to calls by male o 3 met male $\delta^{\prime} 1$ about halfway along her route and advertised and copulated with him. However, before ejaculation took place, she broke of, rushed to male ..:3, and copulated with him.

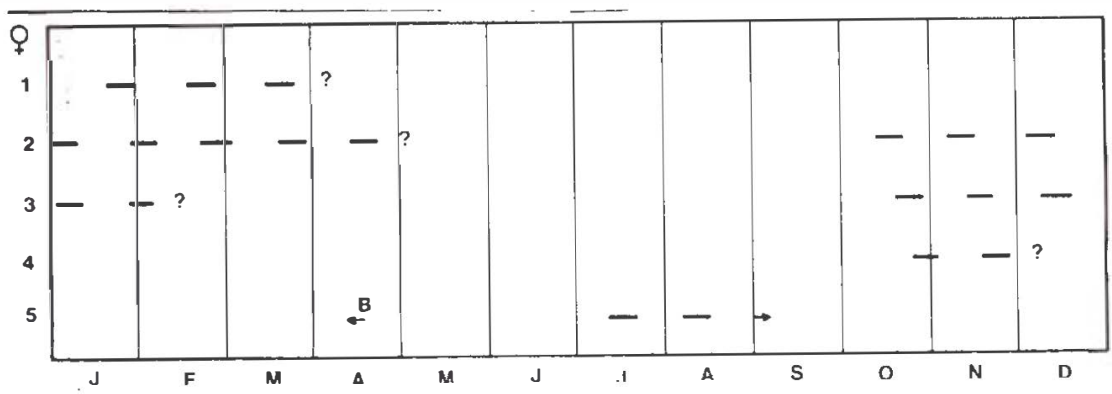

Figure 42 Estrus periods in tive adult females belonging to the spider monkey study group, as observed from May 1977 to May 1978. 1, 2 and 4 are leading females; 1 and 2 were accompanied respectively by a juvenile- 2 temale and a juvenile- 3 male; 3 and 5 are non-leading females without offspring. $\mathrm{B}=$ birth;? = observations not continued. 
The mean duration of obverved complete copulations was $10 \mathrm{mi}-$ nutes $(\mathrm{N}=5)$. During copulation a female is very active, brings ane hand to her ahdomen, moves continuously to and fro on the branch on which copulation takes place, shakes her head restlessly, and regularly looks over her shoulder into the male's face. Her lips are protruded and the eyes half-closed. Sometimes, the female rubs the male's upperleg or back with one hand. At the moment of ejaculation, the female may move a couple of meters with the male hanging behind her. Then the animals separate. In general, three or four copulations take place each day during a female's estrus period. 


\section{CONSERVATION}

Ateles is threatened by hunting, live capture and habitat destruction (Grimwood, 1969; Freese, et al., 1976; Hermández-Camacho and Cooper, 1976; Mittermeier, 1977; Mittermeier and Coimbra-Filho, 1977).

For several reasons, spider monkeys are extremely vulnerable to hunting. Locally, they form an important food item, as they are large and good to eat. The size and noisy habits make them rather easy to locate, track and hunt. They defend themselves trom human intruders by means of an aggressive branchshaking and dropping display. This behavior affords a hunter shooting an entire subgroup out of a tree crown. In addition, they show a very low reproductive rate. Females do not give birth before the age of tive and, thereafter, only once every three to four years. Spider monkey social structure makes them even more vulnerable to hunting, as the group's survival may well depend on the few leading females having the best temporal and spatial knowledge of food sources. Unfortunately, for purposes of life capture, particularly the females carrying an infant will be shot. The infants serve a local pet market. Finally, population density is originally rather low (i.e., 7.1 individuals per $\mathrm{Km}^{2}$.

Habitat destruction is another threat to spider monkey's survi$\mathrm{val}$. Ateles are largely restricted to undisturbed, most heterogeneous lowland rainforests which are going to disappear quickly all over their range.

In general, Ateles is an extremely vulnerable primate that shows little or no adaptability to human intrusion. In Amazonia, hunting is the main cause of decline. In Central-America, it is primarily threatened by habitat destruction. In Surinam, most of the interior is still undisturbed, making the outlook for conservation fairly good. At the moment, Surinam parks and nature reserves protect as much as $5,304 \mathrm{~km}^{2}$ of rain forest hahitat in the interior, most of them in remote and uninhabited areas. When the infrastructure of the interior shall be improved in the near future, the protection of these areas wils be a major problem to conservationists. Hunting pressure will increase and illegal lumber extraction will threaten these areas too. 
Unfortunately, a number of tree species providing spider monkeys, among other animals, with safe sleeping sites and food, produce commercially valuable timber. For these reasons, the national parks and nature reserves will be best protected by so-called buffer zones surrounding them. These areas should lack an infrastructure which, in general, enables more than incidental hunting and logging.

As pointed out before, for many canopy trees and lianas spider monkeys play an important, sometimes perhaps vital role in seed dispersal and escape from seed predation. Local extinction of spider monkeys alone may strongly affect primary forest composition and structure and may cause forest degradation. Unfortunately, this will be noticeable a long time after, since these processes take place gradually. 


\section{DISCUSSION}

\section{The relation between feeding behavior and social organi- zation.}

Several authors have tried to find relationships between habitat in which primate species are found and social organization (Crook and Gartlan, 1966; Eisenberg, et al., 1972; Jolly, 1972; Altmann, 1974; Clutton-Brock, 1974; Struhsaker, 1969; Wilson, 1975). These have met with only very limited success. Results are better when use of environment is correlated with aspects of social organization (Clutton-Brock and Harvey, 1977; Milton and May, 1976). As Rasmussen (1981) points out, proximate studies form rearly the entire data base for primate socioecology (e.g., the studies contained in the volume "Primate Ecology" edited by Clutton-Brock, 1977), whereas only few researchers directly measure effects of environment or use of environment on social interactions. The "social" of primate socioecology is largely missing. Therefore, autecological tield studies describing diet, phenology of food plants, food supply, distribution of food, foraging behavior and social behavior through out the year and in great detail are urgently needed. The present study may serve as an exemple, as will be discussed below.

Spider monkey food specialization involves nutritious (lipid-rich), mostly single-- and large-seeded, mature fruits, which as a rule are available for relatively long periods of time but in small quantities at any given time in a single food plant. This type of fruit seems to have evolved in parallel with the frugivorous animals dependent on it and providing the plant with proper seed dispersal (McKey, 1975). These plants invest much more energy per propagule in a lipid-rich flesh than do plants with watery (sugar-rich), small-seeded berries and figs, or dry, wind-dispersed fruits. The latter generally produce large, mass-ripening fruit crops in order to satiate seed predators and to use a wide array of dispersers feeding on the fruits. However, seed dispersal offered to lipid-rich truits by animals such as spider monkeys involves regular prolonged utilization, a gentle treatment by the disperser's mouth and gut (and some including quicker germination after defecation, e.g. in the case of stones), endochorical transport over considerable distances from the parent tree, dropping in preferred habitat and development of relatively large 
with advanced growth which increases the chance of successfully overcoming the first stage in the plant's life cycle (Van der Pijl, 1969). This strategy has many advantages for the plant but costs are relatively high. In evolutionary terms, this might have been the major reason for many high forest plants with large-seeded, lipidrich fruits to produce asynchronous, slow-ripening fruit crops not successful every year and favoring both the specialized frugivorous animals and its own seed dispersal.

It is true that few fruits of the lipid-rich type are dropped unexploited on the forest floor below the parent plant. They are exploited in a very efficient way by the specialized trugivores dependent upon them. This may be also an explanation for the abundance of terrestrial animals being attracted under trees in which spider monkeys are feeding or have been feeding just before, since these much appreciated lipid-rich fruits are otherwise not available to them.

Asynchronous, prolonged and slow-ripening fruit crops that provide small quantities of mature lipid-rich truits at any given time force large-sized specialized frugivores such as spider monkeys to forage in small subgroups and with relatively short feeding bouts.

In contrast, another sympatric monkey, the bearded saki (Chiropotes satanas chiropotes), shows roughly similar habitat preferences in horizontal and vertical distribution but feeds largely on immature seeds (Van Roosmalen et al., 1981). Ripe fruits and flowers play only a minor role in its diet. It shows a completely different foraging strategy and social organization even though overlap in choice of fruitispecies eaten between the two primates is considerable (i.e., Chiropotes fed upon a total of 78 species of fruits of which 52 were used for its young seeds; most of these fruits were exploited at a mature stage by Ateles).

The bearded saki forages in large, multimale groups consisting of 8-30 or more animals and ranges over large areas (Ayres, 1981; Van Roosmalen, et al. 1981). Foraging routes appeared to be largely determined by spatial cistribution of certain vegetation types, subtypes or phytosociological units of lower rank that offered relatively high densities of few species with edible young seeds. Since the stage of immaturity does not at all seem to be critical to this monkey, seeds are available for many months and in large quanti- 
ties with-in a single food source. This feature sustains the behavior pattern of foraging in large groups.

However, there is some seasonality in the supply of young seeds (Ayres, 1981). It certain vegetation types or plant associations do not offer enough to feed upon together, during the course of a day's foraging activities a group of bearded sakis may up into two or three subgroups and spread over a large area. During this time, they constantly keep mutual contact by means of their extremely loud long calls ("wiché"), while sleeping and traveling between foraging grounds is always performed cohesively by all group members.

This comparison of two sympatric primate species that largely overlap in habitat choice and choice of food species, indicates that social organization among primates may be related to very fine interspecific ecological differences such as preference for the same fruit species at different stages of development.

\section{Comparison of spider monkeys with chimpanzees}

As already noted by Cant (1977), spider monkey social organization shows a remarkable similarity with that of the chimpanzee (Pan troglodytes). Both primates live in distinguishable groups or communities, with a loose, unstable social structure within the group or community and intergroup agonistic behavior (Goodall, 1965, 1968; Reynolds and Reynolds, 1965; Klein, 1972; Bygott, 1974; Wrangham, 1975, 1977; Cant, 1977; Van Roosmalen, 1980, this study). Both in Ateles and Pan, a highly developed knowledge of available food sources seems to be present, and the assumed detailed spatial memory results in economical routes between food sources. Apparently, both species are capable of returning to certain food sources from any location within their range by the shortest possible routes (Wrangham, 1977; Van Roosmalen, 1980, this study).

The ioose, unstable social structure within the group or community seems to be prim related to food specialization (i.e. particulary mature, lipid-rich (ruits) and seasonal varium in foodpatch size Relatively large subgroup or parties form in times of food abundance and sela small ones ave noted in times of food scarcity, perhaps as a refult of increased feeding contion (Azuma and Toyoshima 1962; Reinolds and Reinolds, 1965; Sugiyana, 1973; Nishida, 1974; Wranghan, 1975, 1977; Van Roosmalen, 1980, this study). 
In both species, males perform food-calls or pant-hoots, and foodcalling is positively related to food supply (Goodall, 1965, 1968; Reynolds and Reynolds, 1965; Wrangham, 1975, 1977; Van Roosmalen, 1980, this study). Reynolds and Reynolds (1965) and Eisenberg, et al. (1972) argue that the function of food-calling is to spread information about the location of food sources, and is thereby seen as altruistic. However, food-calling is least frequent when food availability is lowest and when leaming about food sources would be most favorable to other group members. Wrangham (1977) points out that food-calls will tell other chimpanzees that a male has just arrived at a certain food source, which is now being depleted. When giving food long-calls, male Ateles paniscus often are part of relatively large subgroups and joining of subgroups usually does not take place after such food-calls.

The concept of "return time regulation" (Cody, 1974) seems to be applicable here, and directed towards other leading females not in the subgroup containing the calling male. For cropping the new growth, particularly in case of a limited amount of mature fruits, this female is told to drop this food source from her foraging route.

The relation of body size to food patch size is reflected in a relatively high degree of solitary behavoir, higher in chimpanzees, and in relatively small subgroups or parties throughout the year with the only permanent bond formed by females with their offspring. When infants and juveniles are considered together with their mothers, females with or without offspring range more frequently solitary than males, possibly related to their better knowledge of a certain "core area" (Goodall, 1965, 1968; Halperin, 1978; Van Roosmalen, 1980, this study).

Because of the flexible subgroup size in both species, aggressive and submissive behavior within a group or community is infrequent and occurs almost exclusively while feeding.

Individual adults occupy "core areas" within the group or community range, areas most frequently used in a particular part of the year (Wrangham, 1975, 1977; Van Roosmalen, 1980, this study) Male "core areas" are larger than female ones but at least in Ateles females possess a better knowledge of available food sources within these areas, whereas males travel more widely in times of food abundance. 
In both species co-operation between the sexes appears largely unnecessary, except during mating. Therefore, the larger subgroups or parties and wider travel by males in times of lood abundance apparently signify increased reproductive effort. As Wrangham (1977) points out, reproductive competition may be expected to continue between males, since any female will be estrous in the future and will choose a male on the basis of her full knowledge of him.

Observations by Bygott (1974) and Wrangham (1975) on aggressive relations between male chimpanzees of different communities revealed that the relative size of parties is an important factor determining the outcome of the interactions. Wrangham (1975) hypothesized "that the functional consequence of territorial expansion was the acquisition of females, and tha the formation of large parties may be viewed as improving the reproductive success of a male community through its increased probability of winning teritorial encounters and hence females". This hypothesis also seems to be applicable to spider monkey community. At territorial boundary conflicts, male Ateles paniscus performed so-called alarm long calls, thereby attracting nearby parties to the spot for sharing the longdistance agonistic behavior of shaking and breaking oft twigs, branches and boughs and performing "ook-barking" towards the members of the neighboring group.

While males stay within the perimeter of the group's range, females occasionally do visit members of neighboring groups and emigration of females, particularly young non-leading females, seems to occur. Malemale dominance exists. Males cooperating in territo rial defence seem to be related. This kind of social systern permits males to defend large temitories, including ranges of several females, by cooperating in all-male parties during territorial defence pa. trols and boundary conflicts with other groups (Goodall, 1965, 1968; Bygott, 1974; Wrangham, 1975, 1977; Riss and Goodall, 1977; Pusey, 1978; Van Roosmalen, 1980, this study). 


\section{SUMMARY}

This study describes habitat and food choice of the Surinam black spider monkey, Ateles paniscus paniscus. It clarifies complex temporal and spatial effects of food sources on the behavior of a group of spider monkeys in a 350 ha study area in central Surinam in terms of food category, food plant identity and phenology and in terms of quantity, density and dispersion of the nost irisportant of these food sources. It recognizes the fundamental s. :portance of mature fruit-feeding to spider monkey foraging strategy and discusses implications of diet for social behavior. From a conservation point of view, this study is essential since it emphasizes the extreme vulnerability of the spider monkey to both hunting and habitat destruction. In addition, it provides detailed information on spider monkey habitat choice and dietary requirements that is urgently needed in order to assess both potential and established protected areas. The spider monkey serves as an important "indicator" species, reflecting the degree of disturbance to Neotropical rain forests.

1. In the Voltzberg region, Ateles paniscus occurs at a density of 7.1 individuals per $\mathrm{km}^{2}$, or 8.2 individuals per $\mathrm{km}^{2}$ when only suitable habitat is considered. Biomass ranges between 0.4 and 0.5 $\mathrm{kg} / \mathrm{ha}$ depending on the home range figure chosen.

Forest types found in the Raleighvallen-Voltzberg region are described structurally. Dry evergreen forest types are divided into tropical rain forest (i.e., high rain forest, low rain forest, riverbank high forest), mountain savanna forest, and liane forest. Wet forest types are divided into swamp forest and marsh forest. Finally, a xeromorphic vegetation, "rocksavanna", is distinguished.

2. The present study was conducted in the RaleighvallenVoltzberg Nature Reserve, a 56,000 ha protected area located on the east bank of the Coppename River in central Surinam. A 350 ha study area was gridded and a vegetation map of the area was made. During the first year, the study was focussed on the synecology of all eight Surinam primates living in the area. In addition, a group of spider monkeys were habituated, data on their general behavior were collected, and the perimeter of the group's home range was roughly determined. During the second year, the study was con- 
centrated mainly on the autecology of spider monkeys. During the entire field period, the phenology of especially food plant species was monitored. In cooperation with a native tree specialist, approximately 10,000 individual trees and lianes belonging to 120 species of food plant were tagged and plotted in special maps in a 205 ha area, that was the most important part of the Ateles group range.

Some other localities were investigated in Surinam during the general survey, which added data to the distribution map and the habitat table.

3. Among the eight Surinam monkey species, Ateles paniscus is the most restricted in habitat. In the Voltzberg region, it occurs almost exclusively in high forest $(92.6 \%)$, intrequently enters edge habitats ( $14.9 \%)$, and is found primarily in the upper levels of the canopy and in emergents (72.3\%). The understory is rarely used $(0.8 \%)$, and the lower extreme of its vertical range appears to be 12 meters. Among the seven major forest types available in the Raleighvallen-Voltzberg region, spider monkeys are observed only in high rain forest, mountain savanna forest, pina swamp forest and riverbank high forest.

4. A total of 207 food plant species are used, of which $68.1 \%$ are trees. The most important food plant families are Moraceae and Mimosaceae, both in terms of number of tood species and percentage of total feeding records. Ateles paniscus is mainly frugivorous and feeds on 171 species of fruit, 33 species of flower and 28 species of leaf. Mature fruits make up $96 \%$ of the total number of fruit feeding records. Occasional feeding upon insects (i.e., termites and caterpillars) definitely takes place. The average annual food intake is $82.9 \%$ fruits, $6.4 \%$ flowers, $7.9 \%$ flush leaves, $1.7 \%$ bark and $1.0 \%$ miscellaneous (i.e., rotten palm sheaths, pseudobulbs, aerial roots, honey, insects). Monthly variation in food choice shows a strong correlation with phenology. During the first part of the long dry season (i.e, from July to September), a period of low fruit supply, the monkey compensates its diet with relatively high percentages of both flowers and flush leaves. During the long wet season (i.e., from March to June), fruit abundance results in very low percentages of both flowers and flush leaves in the diet. Ripe fruits always seem to be preferred. Young seeds play a minor overall role in the diet, except for the period May-June. By ingesting large quantities of young seeds 
rich in protetin and fat during the peak of the long wet season, the monkeys seem to stock up on energy for the coming months of food scarcity (i.e., from July to October).

Foraging behavior in spider monkeys differs strikingly with the seasons. During the long wet season when fruit is abundant, activity budgets are increased. This results in large day ranges (with a maximum length of about 5,000 meters), prolonged feeding times, short resting times and daily utilization of many food sources (i.e., particularly mature fruits). Foraging often takes place in relatively large subgroups that break up and reassemble regularly, the subparties using partly different food sources but following roughly similar itineraries. During the long dry season, when fruit supply is low and food scarcity (or in some years even food shortages) exists, activity budgets are lowered to a minimum. This results in short day ranges (with a minimum of about 500 meters), prolonged resting times, short feeding times (i.e., few relatively long feeding bouts), daily utilization of only a few food sources and much higher percentages of flowers and flush leaves in the diet. Finally, mean subgroup size is decreased strongly.

Spider monkeys live in medium-sized groups that fragment into widely dispersed subgroups of varying composition. Daily itineraries and activity patterns of a subgroup are mainly determined by a so-called leading (usually older) female with or without off spring, or sometimes by two leading females altemating responsabilities. These females appear to possess the best knowledge of certain parts of the group home range, the so-called core-areas, and seem capable of fixing beforehand an economic foraging route for the day that can include from 8 to 30 different food sources. By regularly monitoring potential food sources as to their stage of maturity and using a highly developed spatial and temporal memory, these females appear to be able to incorporate maturing food sources into their foraging routes just after they become available. The interval between subsequent visits to a particular food source appear to be species-specific and depends on the maturation rate of the fruit. In some species, the last stage of maturation passes rapidly, offering the monkeys a large enough food supply to enable an entire subgroup to feed simultaneously in the same tree. Many species, however, are exploited in $2-4$ day cycles, some in 5-8 dây 
or even longer cycles. Spider monkeys appear to select for variety, using daily an average of about 14 different tood items that comprise about a quarter of the average monthly number of food items used. Of these, three or four food items are most important in terms of both total time spent feeding upon them and estimated weight of food ingested.

Ateles plays an important role as a dispersal agent for many plant species, and for some species it even seems to be the only seed disperser in the Voltzberg region. Endochorical seed dispersal by spider monkeys took place in 138 plant species (accounting for $93.5 \%$ of all fruit feeding records), seed dropping was recorded in 10 species (2.7\%) and seed predation in 23 species (3.7\%). Ateles belongs to the category of "specialized frugivores" that derive all or most of their supplies of carbohydrate, lipid and protein from fruits. Large-seeded, nutritious (lipid-rich) truits seem to have evolved parallel with specialized frugivores as their principal dispersal agents, resulting in a greater quality of dispersal than that found in small-seeded, low-nutritious (sugar-rich) fruits which are usually dispersed by a wide array of both "opportunistic" and "specialized" fruit-eating animals. This coevolutionary pattern (i.e., the high nu. tritive content of the flesh in large-seeded fruits) may be demonstrated in families such as Burseraceae, Capparaceae, Loganiaceae, Meliaceae, Myristicaceae, Palmae, Sapindaceae and Sapotaceae, all producing important fruits for spider monkeys. Among the 166 plant species with edible fruits used by the spider monkeys in the Voltzberg region, about $80 \%$ produce lipid-rich and large-seeded fruits. Sugar-rich, small-seeded truits (i.e., particularly berries and tigs) make up only $20 \%$ of the fruit species in the diet, and are exploited only incidentally on the way from one lipid-rich fruit source to another. They almost never appear to influence the daily foraging route, and are not regularly revisited. These species often produce mass-ripening fruit crops on which the monkeys cannot greatly depend. However, the fruiting seasons of the larger-seeded fruits in general last relatively long because of more or less asynchronous fruit maturation within and between individuals of the species. This pattern may have evolved because the small number of specialized dispersal agents can be easily overloaded. The competition between these plant species for dispersal offered by a small number of dis- 
persers may have evolved in lengthy, displaced, but broadly overlapping fruiting seasons required to supply specialized frugivores the year round.

5. A group of Ateles paniscus usually consists of 15-20 individuals, although all members may never be observed together at the same place. A group fragments into several subgroups of varying composition, a female with offspring ranging in age from zero to about five years being the only persistent subgroup observed. The annual home range of the spider monkey study group in the Voltzberg area covers 255 hectares, of which 220 hectares offer suitable habitat. Daily travel paths range between 500 and 5,000 meters in length depending on the subgroup size and composition, the weather, the season and the distribution of particular important food sources. Sex ratio of adult males to adult females is $1: 2(-3)$. The adult males of a group defend a teritory with clearcut boundaries, whereas females sometimes visit neighboring groups and even may emigrate.

Spider monkey social system is characterized by its flexible grouping behavior and seems to have evolved parallel with its food choice concerning mainly lipid-rich, large-seeded fruits. The supply of this type of lood varies greatly with the seasons and maturation rates within and between individual fruitings plants are generally rather slow and asynchronous. Moreover, individual mature fruits of this type are available to the monkey only for a brief period of time. After becoming, mature, most inuits scon drop to the ground or, when dehiscent, become available to birds. These food plants seldom offer enough for more than three adult spider monkeys to feed upon together. Consequently, subgroups consisting of $\leqslant 3$ individuals are the most frequently encountered throughout the year. However, high densities of particular food plants fruiting during the long wet season enable spider monkeys to forage in much larger subgroups that may contain two leading females and may range in size up to nine independentty locomoting individuals simultaneously using roughly the same itineraries but exploiting somewhat different food sources. As a result, during this season intragroup social interactions are much more frequent.

During the following long dry season, when lipid-rich fruit is scarce (and in some years even severe food shortages may exist), 
the mean subgroup size decreases sharply. Leading-female core-areas are separated and show almost no further overlap throughout the season. Non-leading females and males still may assemble into subgroups led by a female to share ecological knowledge of food sources but they do so less frequently. Daily travel paths drop strikingly in length and the animals are more silent. Both of these factors contribute to a lower chance of mutual encounter. Activity budgets are decreased, particularly in males and non-leading females. The better knowledge of available food possessed by lea ding females may favor them for survival, especially during the long dry season. Also, this may be a reason why leading females seem more successful in rearing offspring than do non-leading females, and why mortality in adult males, on the basis of sex ratio, seems to be higher:

At the end of the long dry season when preferred fruit is still in low supply, relatively frequent feeding upon flush leaves and flowers again sustains foraging in larger subgroups by providing large amounts of food at a given time in large-crowned flushing or flowering food trees.

Adult males do possess core-areas and these usually are larger than those of leading females and even may include core-areas of two leading females. Adult males cooperate in territorial defence patrols and long-distance agonistic behavior (e.g., boundary conflicts). In this way, spider monkey males are able to defend more females than would be possible in a social system with cohesive bisexual groups, such as those found in many other primates.

The spider monkey social system is unusual among primates and shows the most striking similarities to that of the chimpanzee (Pan troglodytes). Both species are largely frugivorous, and have a loose, unstable social structure within distinguishable groups. Subgroup size varies seasonally in relation to food supply and usually is small. Individual adults occupy "core-areas" and show capacity for and continual use of a detailed, highly developed spatial memory. Dominance behavior and sexual behavior is also remarkably similar in the two species.

6. Ateles is a highly vulnerable primate showing little or no adaptability to human intrusion. Hunting, live capture and habitat destruction are the main threats to its survival. Spider monkeys are considered a very important component of lowland tropical rainfo- 
rest ecosystem. Being one of the principal dispersal agents for plants with larger-seeded, usually lipid-rich fruits, a common phenomenon in primary forest canopy trees and lianas, local extinction of Ateles will probably cause long-term forest degradation. It is proposed to protect Ateles in national parks and nature reserves surrounded by buffer zones without an infrastructure preventing them from large-scale hunting and habitat destruction. 


\section{REFFRENCES}

ALTMANN, J.: Observational study of behavior. Sampling methods. Behavior 49: 227-267 (1974)

AIVAREZ DEL TORO, M.: Los mammiferos de Chiapas. Universidad Autonoma de Chiapas, Tuxtla Gutierrez, Chiapas, Mexico (1977).

AYRES, J.M.: Observaçōes sobre a ecologia e o comportamento dos cuxiús (Chiropotes albinasus e Chiropotes satanas, Cebidae: PRIMATES) (1981).

AZUMA, S. and TOYOSHIMA, A.: Progress report of the survey of chimpanzees in their natural habitat, Kabogo Point Area, Tanganyika. Primates 3:61-70 (1962).

BAKKER, I.P.: Quelques aspects du problème des sédiments corrélatifs en climat tropical humide. Zeitschr. f. Geomorph. 1:1-43 (1957).

BAKKER, J.P.: Zur Entstehung von Pingen, Oriçangas und Dellen in den feuchten Tropen, mit besonderer Berücksichtigung des Voltzberggebietes (Surinam). Abh. Geogr. Inst. d. Fr. Univ. Berlin, 5:7-20 (1957).

BARTIET, E.: Notes on the monkoys of castem Peru. Proc. Zool. Soc. London: 217-220 (1987).

BEARD, J.S.: The classification of tropical American vegetation types. Ecology 36:89-100 (1955)

BERG, C.C. and DEWOLF, G.P: Moraceae. Flora of Suriname Vol, V, Part l (E.J. Brill, Leiden) (1975).

BERNSTEIN, I.S.; BALCAEN, P.; DRESDALE, H.; GOUZOULES; H.; KAVANAGH, M.; PATTERSON, T., and NEYMAN-WARNER, P.: Differential effects of forest degradation on primate populations. Primates 17:401-411 (1976).

BRAAK, C.: Het klimaat van Nederlandsch West-Indië. Kon. Ned. Met. Inst. 1-2, Med. en Verh. 36: 1.120 (1935).

BRAMBLETT, C.A.; BRAMBLETT, S.S; COELHO, A.M., and QUICK, L.B.: Party composition in spider monkeys of Tikal, Guatemala. A comparison of stationary vs. moving observers. Primates 21 (1): 123-127 (1980).

BYGOTT, J.D.: Agonistic behavior' and dominance in wild chimpanzees, Ph. D. thesis, University of Cambridge (1974).

CANT, J.G.H.: Ecology, locomotion and social organization of spider monkeys (Ateles geoffroyi). Unpublished doctoral dissertation, University of California, Davis (1977).

CANT, J.G.H.: Population survey of the spider monkey Ateles geoffroyi at Tikal, Guatemala. Primates 19 (3): 525-535 (1978).

CANT, J.G.H.: Dispersal of Stemmadenia donnel.smithii by birds and monkeys. Biotropica 11: 122 (1979).

CARPENTER, C. R.: Behavior of red spider monkeys (Ateles geoffroyi)) in Panama. J. Mammal. 16: 171-180 (1935). Also in CARPENTER Naturalistic behavior of nonhuman primates.

CHIVERS, D.J.: On the daily behavior of howling monkey groups. Folia primat. 10:48-102 (1969).

CHIVERS, D.J.: The siamang in Malaya. A field study of a primate in tropical rain forest. Contributions to primatology 4: 1-335 (Karger, Basel 1974).

CHIVERS, D.J.: The ding behaviour of siamang (Symphalangus syndactylus); in CLUTTON- 
BROCK Primate ecology; studies of feeding and ranging behaviour in lemurs, monkeys and apes (Academic Press, London 1977).

CLUTTON-BROCK, T.H.: Primate social organisation and ecology. Nature (Lond.) 250:539-542 (1974).

CLUTTON-BROCK, TH.: Feeding behaviour of red colobus and black and white colobus in East Africa. Folia primat. 23: 165-207 (1975).

CLUTTON-BROCK, T.H.: Some aspects of intraspecific variation in feeding and ranging behaviour in primates; in CLUTTON-BROCK Primate ecology: studies of feeding and ranging behaviour in lemurs, monkeys and apes (Academic Press, London 1977).

CLUTTON-BROCK, T.H.: Methodology and measurement. Appendix 1; in CLUTTON-BROCK Primate ecology: studies of feeting and ranging behaviour in lemurs, monkeys and apes (Academic Press, London 1977).

CLUTTON-BROCK, T.F. and HARVEY, P.H.: Species ditterences in feeding and ranging behaviour in primates; in CLUTION-BROCK Primate ecology: studies of teeding and ranging behaviour in lemurs, monkeys and apes (Academic Press, London 1977).

CODY, M.L.: Optimization in ecology. Science 183: 1156-1164 (1974).

COEL.HO, A.M.: Socio-bioenergetics and sexual dimorphism in primates. Primates 15: 263-269 (1974).

COELHO, A.M.: Social organization and resource availability in Guatemalan howler and spider monkeys: a sociobioenergetic analysis. Paper presented at the 44 th Annual Meeting of the American Association of Physical Anthropologists, Denver, Colorado (1975).

COEL.HO, A.M.; BRAMBLETT, C.A.; QUICK, L.B., and BRAMBLETT, S.S.: Hesource availability and population density in primates: a sociobioenergetic analysis of the energy budgets of Guatemalan howlex and spider monkeys. Primates 17: 63-80 (1976).

COLI.IN, C.D.: Fauna Surinamensis. Thesis, Upsala (1822).

CROOK, 3.H. and GARTLAN, J.S.: Evolution of primate sacieties. Nature 210: 1200-1203 (1966).

CHUZ LIMA, E. DA: Mamíteros da Anazônia. Introduçāo Geral e Primatas. Museu Paraense Emilio Goeldi de Historia Natural a Ethnografia, Belém do Pará, Brazil (1944).

DARE, R.: The social behavior and ecology of spider monkeys, Ateles geoffroyi, on Barro Colorado Island; unpublished doctoral dissertation, University of Oregon (1974).

DARE, R.: Food-sharing in tree-ranging Ateles geoffroyi (red spider monkeys).Lah. Prim. News). 13 (1): 19-21 (1974).

DARE, $R$.: The effects of fruit abundance on movement patterns of freeranging spidex, monkeys, Ateles geoffroyi. Paper presented at the 44th Annual Meeting of the American Association of Physical Anthropologists, Denver, Colorado. Abstract in Amer. J. phys. Anthrop. 42: 297 (1975).

DAVIS, H.: Field checklist of birds of the Guianas. Florida Audubon Society (1966).

DURHAM, N.M.: Effects of altitude differences on group organization of wild black spider monkeys (Ateles paniscus); Proceedings 3rd int. Congr. Primat., Zurich, vol. 3, pp. 32-40 (Karger Basel 1971).

DURHAM, N.M.: Some ecological, distributional, and group behavioral features of Ateilnae in southern Peru: with comments on interspecific relations; in TUTTLE Socioecology and psychology of primates, pp. 87-103 (Mouton, The Hague 1975).

EISENBERG, J.F.: Reproduction in two species of spider monkeys, Ateles fusciceps and $A$. geoffroyi. J. Mammal. 54: 955-957 (1973). 
EISENBERG, J.F::Communication mechanisms and social integration in the black spider monkey, Ateles fusciceps robustus, and related species. Smithson. Contrib. Zool. 213: 1.108 (1976).

EISENBERG, J.F.: Communication mechanisms in Now World Primates with special reference to vocalizations in the black spider (Ateles fusticeps zobystus); in KRAMES, PLINER and ALLOWAY aggression, dominance and individual spacing, pp.31-49 (Plenum Pulb. Corporation, 1978).

EISENBERG, J.F. and KUEHN, R.: The behavior of Ateles geoffroyi and related species. Smithson. Misc. Collns. 151(8): 1-63 (1966).

EISENBERG, J.F.; MUCKENHIRN, N.A., and RUDRAN, R.: The relationship between ecology and social structure in primates. Science 176: 863-874 (1972).

EISENBERG, J.F. and THORINGTON, R.W.: A preliminary analysis of a Neotropical mammal fauna. Biotropica 5 (3): 150-161 (1973).

ELLEFSON, J.O.: $\AA$ natural history of gibbons in the Malay Penunsula; in RUMRAUGH The gibbon and the siamang, vol. 3: 1-136 (Karger, Basel 1972).

FANSHAWE, D.B.: The vegetation of British Guiana, a preliminary review. Imp. For. Inet. Oxf., Inst. Pap. 29 (1952).

FANSHAWE, D.B.: Forest types of British Guiana. Carib. For. 15: 73-111 (1954).

FLEAGLE, J.G.: Locomotion and posture of the Malayan siamang and implications for hominoid evolution. Folia primat. 26: 245-269 (1976).

FOTOGEOLOGISCHE KAART VAN SURINAME: Ministerie van Opbouw, Paramaribo, Suriname (1966).

FOODEN, I.: Stomach contents and gastro-intestinal proportions in wild-shot Guianan rnonkeys. Amer. J. phys. Anthrop. 22: 227-232 (1964).

FREESE, C.: A census of non-human primates in Peru; in Primate censusing studies in Peru and Colombia, pp. 17-42, Pan American Health Organization, Washington, D.C. (1975).

FREESE, C.: Censusinc 'Alouatta palliata, Ateles geoftroyi and Cebus capucinus in the Costa Rican dry forest; in THORINGTON and HELTNE Neotropical primates: field studies and conservation, pp. 49, National Academy of Sciences, Washington, D.C. (1976).

FREESE, C.: Population densities and niche separation in some Amazonian monkey communities. Diss. Abstr. Int. B38: $5732-5733$ (1978).

FREESE, C.: NEVILlE, M., and CASTRO, R.: The conservation status of Peruvian primates. Lab. Primate Newsletter 15 (3): 1-9 (1976).

FREESE, C.g. OPPENHEnMER J.R.: The capuchin monkeys, genus Cebus; in COMMBRA-FILHO and MTTERM Ecology and behavior of nectropical primates, pp. 331.390, Academia Brasileira de Ciências, Rio de Janeiro.

GAUTER-HION, A. and GAUTIER, J.P.: Ecological niche and species diversity in sympa. tric forest monkeys. La Terre et la Vie 33: 493-507 (1979).

GEYSKES, D.C.: Het dierlijk voedsel van bosnegers aan de Marowijne. Vox Guyanae 1(2): $61-83$ (1954).

GEYSKES, D.C.: Met de Oajana's op stap. Vox Guyanae 3(5-6): 193-300 (1957)

GEYSKES, D.C.: De expeditie naar de Tafelberg in 1958. Vox Guyanae 3(4): 1-52 (1959).

GEYSKES, D.C.: Documentary information about the Surinam or Wama Indians. Nieuwe WestIndische Gids 3: 260-285 (1970).

GOODALL, J.: Chimpanzees of the Gombe Stream Reserve; in DEVORE Primate hahavior. pp. 425-473 (Holt, Rinehart \& Winston. New York 1965).

GOODAL, 3 .: The behaviour of free-living chimpanzees in the Gombe Stream Reserve. Animal. Behav. Monogr. 1(3): 165-311 (1968). 
GOODAL, J.: Cultural elenents in a chimpanzee community. Symp. IVth int. Congr. Primat, vol. 1, pp. 144-184 (Karger, Basel 1973).

GOODMAN, L. and WLSLOCKI, G.B.: The cyclical tuterine bleeding in a New World monkey (Ateles geoffroyi). Anatomical Record 61: 379-387 (1935).

GRIMWOOD, I.R.: Notes on the distribution and status of some Pleruvian mammals 1968. Special Publication No 21 American Committee for International Wild Life Protection and New York Zoological Society (1969).

GROVES, C.P. Phylogeny and classification of primates; in FIENNES Pathology of Simian primates, part 1 (Karger, Basel 1972).

HALPERIN, S.D.: Grouping patterns of chimpanzees in Gombe National Park, Tanzania; in HAMBURG and GOODALI Perspectives on human evolution, vol. IV. The behaviour of great apes (Benjamin, London and New York, 1978).

HARTLEY, P.H.T: An ecological study of the feeding habits of English titmice. J. Anim. Ecol. 22. $261-288$ (1935).

HAVERSMIDT, F: The birds of Surinam (Oliver \& Boyd Ltd., Edinburgh 1967).

HELTNE, P.G. and KUNKEI, L.M.: Taxonomic notes on the pelage of Ateles paniscus paniscus, A. p. chamek (sensu Kellogg and Goldman, 1944) and A. fusciceps rufiventris (= A. f. robustus Kellogg and Goldman, 1944). J. Med. Primat. 4: 83-102 (1975).

HELTNE, P.G.; FREESE, C. and WHITESDES, G.: $A$ tield survey of non-human primate populations in Bolivia. Pan American Health Otganization, Washington, D. C. (1975).

HELTNE, P.G. and THORINGTON, A.W. Problems and potentials for primate biology and conservation in the New World; in THORINGTON and HELTNE Neotropical primates: field studies and conservation, pp. 110-124, National Academy of Sciences, Washington, D. C. (1976).

HERNÁNDEZ.CAMACHO, J. and COOPER, R.W. The non-human primates of Colombia; in THORINGTON and HELTNE Neotropical primates: field studies and conservation, pp. 35-69, National Academy of Sciences, Washington. D.C. (1976).

HERSHKOVITZ, P.: Mammals of northern Colombia. Preliminary report n ${ }^{\circ} 4$. Monkeys (Primates), with taxonomic revisions of some forms. Proc. U. S. Nat. Mus. 98: 323427 (1949).

HERSHKOVITZ, P.: Type localities and nomenclature of some American primates, with remarks on secondary homonyms. Proc. Biol. Soc. Washington 71: $53-56$ (1958).

HERSHKOVITZ, P.: The evolution of mammals on southern continents. VI. The recent mammals of the Neotropical region: zoogeographical and ecological review. Quart. Rev. Biol. 44: 1-70 (1969).

HERSHKOVITZ, P.: Notes on New World monkeys. Int. Zoo Yb. 12: 3-12 (1972).

HERSHKOVITZ, P.: Living New World monkeys (Platyrrhini) (University of Chicago Press, Chicago 1977).

HILL, W.C.: Primates, Comparative anatomy and taxonomy, vol. V: Cebidae, part B (University Press, Edinburgh 1962).

HLADIK, A. and HI.ADIK, C.M.: Rapports trophiques entre végétation et primates dans la forêt de Barro Colorado (Panama). La Terre et la Vie 23: 25-117 (1969).

HLADIK, C.M.: L'Atèle de Geoffroy, ce singe-araignée. Science et Nature 111: 1-11 (1972)

HLADIK, C.M.: Alimentation et activité d’un groupe de chimpanzés réintroduits en forêt gabonaise. La Terre et la Vie 27: 343-413 (1973).

HLADIK, C.M.:'Ecology, diet and social patterning in old and New World primates; in TUTTLE Socioecology and psychology of primates, pp. 3-35 (Mouton, The Hague 1975). 
HLADIK, C.M.: Chimpanzees of Gabon and chimpanzees of Gombe; some comparative data on the diet; in CLUTTON.BROCK Primate ecology: studies of feeding and ranging in lemurs, monkeys and apes (Academic Press, London 1977).

HLADIK, C.M.; HLADIK, A; BOUSSET, J.; VALDEBOUZE, P.; VIROBEN, G., and DELORTLAVAL, J.: Le régime alimentaire des Primates de $l^{\prime}$ île de Barro-Colorado (Panama). Résultats des analyses quantitatives. Folia primat. 16: 85-122 (1971).

HOLTHUIIZEN, A.M.A. and BOERBOOM, J.H.A.: The Cecropia seedbank of the Surinam iowland rain forest. Biotropical 14 (1): 62-68 (1982).

HOOGMOED, M.S.: Notes on the herpetofauna of Surinam 1. - Itinerary af a herpetological collecting trip to Surinam in 1968. Zoöl. Meded., Leiden 44(4): 47.73 (1969).

HUSSON, A.M.: Notes on the primates of Surinam. Studies on the fauna of Suriname and other, Guyanas I: $13-40$ (1957).

HUSSON, A.M.: The mammals of Suriname. Zoöl. Monogx. Hijksmuseum Nat. Historie N. 2 (Brill, Leiden) (1978).

IZAWA, K.: Foods and feeding behaviour of monkeys in the upper Amazon basin. Primates 16: $295-316$ (1975).

IZAWA, K.: Group sizes and compositions of monkeys in the upper Amazon basin. Primates 17(4): 367.399 (1976).

IZ.AWA, K ; KIMURA, K., and NIETO, A.S.: Grouping of the wild spider monkey. Primates 20(4): 503-512 (1979).

JANSON, C.H.: Ecology and population densities of primates in a Peruvian rainforest. Submitted in partial fulfillment of the B.A. degree, Department of Biology, Princeton University, Princeton, New Yersey (1975).

JANZEN, D.H.: Seed-eaters versus seed size, number, toxicity and dispersal. Evolution 23: I-27 (1969)

JANZEN, D.H.: Seed predation by animals. Ann. Rev. Ecol. Syst. 2: 465-492 (1971).

JENKINS, R.: Ecology of three species of Saltators, with special reference to their frugivorous diet. $\mathrm{Ph}$. D. thesis, Harvard University (1969).

JOLLY, A.: The evolution of primate behavior (Macmillan, New York and London 1972).

KAPPLER, A.: The monkeys of Dutch Guiana. Pop. Sci. Monthly 32 (3): 396-400 (1888).

KELLOGG, R. and GOLDMAN, E.A.: Review of the spider monkeys. Proc. U.S. Nat. Mus. 96: $1-45$ (1944).

KIESTER, A.R. and SLATKIN, M.: A strategy of movement and resource utilization. Theor. Pop. Biol. 6(1): 1-20 (1974).

KLEIN, L.L.: Observations on copulation and seasonal reproduction of two species of spider monkeys, Ateles belzebuth and A. geoffroyi. Folia primat. 15: 233-248 (1971).

KLEIN, L.L.: The ecology and social organization of the spider monkey, Ateles belzebuth. Unpublished doctoral dissertation, University of California, Berkeley (1972).

KLEIN, L.L.: Agonistic behavior in neotropical primates; in HOLLOWAY Primate aggression, teritoriality, and xenophobia. A comparative perspective, pp. 77-122 (Academic Press, New York and Iondon 1974).

KLEIN, L.L. and KLEIN, D.J.: Aspects of social behavior in a colony of spider monkeys, Ateles geoffroyi, at the San Francisco Zoo. Int. Zoo Yb. 11: 175-181 (1971).

KLEIN, L.L. and KLEIN, D.J.: Observations on two types of neotropical primate intertaxs associations, Amer, J. phys. Anthrop. 38: 649-653 (1973).

KLEIN, L.L and KIEIN, D.J.: Social and ecological contrasts between four taxa of neotropical primates; in TUTTLE Socioecology and psychology of primates, pp. 59-85 (Mouton, The Hague 1975). 
KIEIN, L.L. and KIEIN, D.J.: Neotropical primates: aspects of habitat usage, population density and regional distribution in La Macarena, Colombia; in THORINGTON and HEJTNE Neotropical primates: field studies and conservation, pp. 70-79 (National Aca. demy of Sciences, Waskington, D.C. 1976).

KIEIN, L.L. and KLEIN, D.J.: Feeding behavior of the Colombian spider monkey, Ateles belzebuth; in CLUTTON-BROCK Primate ecology: studies of feeding and ranging behaviour in lemurs, monkeys and apes (Academic Press, London 1977).

LEEFI.ANG, E.C., KOLADER, J.H, and KROONENBERG, S.B.: Suriname in gecgratisch perspektiel (Bolivar Editions, Paramaribo 1976).

LENSEI.INK, L.: De jachtopbrengst in een Surinaams Trio-dorp. De Surinaamse Landbouw 20 (3): $37-41$ (1972).

LINDEMAN, J.C.: The vegetation of the coastal region of Suriname. The vegetation of Suriname, vol. 1, part l (Kemink \& Son, Utrecht 1953).

LNDEMAN, J.C. and MOOLENAAR, S.P.: Preliminary survey of the vegetation of northern Surinama. The vegetation of Suriname, vol.1, part 2, pp. $1-45$ (Kernink \& Son, Utrecht 1959).

MCKEY, D.: The ecology of coevolved seed dispersal systems; in GILBERT and RAVEN Coevolution of animals and plants (University of Texas Press 1975).

MIITON, K.: Distribution patterns of tropical plant foods as an evolutionary stimulus to primate mental development. Amer. Anthrop. 83: 534-548 (1981).

MIITON, K.: Estimates of reproductive parametere for freeranging Ateles geoffroyi, Primates, 22(4): 574-57.

MILION, K. and MAY, M.L.: Body weight, diet and home range area in primates. Nature 259: 459-462 (1976).

MTTANI, J.C. and RODMAN, P.S.: The relation of ranging pattern and bome range size to defendability, with an analysis of tenitoriality among primate species. Behav. Ecol. Sociobiol. 5: 241-251 (1979).

MITTERMEIRR, R.A.: Group activity and population dynamics of the howler monkey on Barro Colorado Island. Primates 14: 1-19 (1973).

MTTERMEIER, R.A.: Distribution, synecology and conservation of Surinam monkeys. Unpublished doctoral dissertation, Harvard University, Cambridge, Mass. (1977).

MITTERMEIER, R.A.: Locomotion and posture in Ateles geoftroyi, and Ateles panis cus Folia primat. 30:161-193.

MITTERMEIER, R.A. and COIMBRA-FILHO, A.F.: Primate conservation in Brazilian Amazonia; in PRINCE RAINIER and BOURNE Primate conservation, pp. 117-166 (Academic Press, New York 1977).

MTTTERMEIER, R.A. and FLEAGLE, I.G.: The locomotor and postural repertoires of Ateles geoffroyi and Colobus guereza, and a reevaluation of the locomotor category semibra. chiation. Amer. J. phys. Anthrop. 45(2): 235-255 (1976).

MITTERMEIER, R.A. and FLEAGLE, J.G.: Locomotor behavior, body size and comparative ecology of seven Surinam monkeys. Amer. J. phys Arthrop. 52: 301-314 (1980).

MITTERMEIER, R.A. and MITON, K.: Jungle jackpot. Animal Kingdom 79 (6): 26-32 (1976).

MITTERMEIER, R.A. and ROOSMALEN, M.G.M. VAN: Preliminary observations on habitat utilization and diet in eight Surinam monkeys. Folia primatol. 36: 1-39 (1981).

MITTERMEIER, R.A. and ROOSMALEN, M.G.M. VAN: A synecological study of Surinam monkeys. Advances in Herpetology and Evolutionary Biology: 521 - 534 (1982).

MozuNO, A.: Four Cebidae species. How to make their living in Orinoco. Monkey, 20 (1-2): $38-49$ (1976). 
MOPTON, E.S. On the evolutionary advantages and disadvantages of truit eating in tropical birds. Amer. Natur. 107: 8-22 (1973).

MOYNIHAN, M.: Some behavior patterns of platyrthine monkeys II. Saguinus geoffroyi and some other tamarins. Smithson. Contrib. Zool. 28: 1-77 (1970).

MOYNIHAN, M.: The New World primates (Princeton University Press, Princeton, New Jersey 1976).

MUCKENHIRN, N.A.; MORTENSEN, B.K.; VESSEY, S: FRASER; C.E.O, and SINGH, B.: Report on a primate survey in Guyana, July-October, 1975. Pan American Health $\mathrm{O}_{2}$ ganization, Washington, D.C. (1975)

NAPIER.: Catalogue of primates in the British Museum (Natural History), part I : Families Callitrichidae Cebidae. British Museum Natural History (1976).

NAPIER, J.R. and NAPIER, P.H.: A handbook of living primates (Academic Press, London and New York 1967).

NEVILIE, M.K.: Census of primates in Peru; in First interamerican conference on conservation and utilization of American non-human primates in biomedical research, pp. 19-29. Pan American Health Organization, Washington, D.C. (1976).

NEVILLE, M.K.; CASTRO, N.; MARMOL, A., and REVIILA, J. Censusing primate populations in the reserved area of the Pacaya and Samiria Rivers, Department loreto, Peru. Primates 17: 151-181 (1976).

NISHIDA, T: The social group of wild chimpanzees in the Mahali Mountains. Primates 9: 167-224 (1968).

NISHIDA, T.: Ecology of wild chimpanzees; in OHTSUKA, TANAKA and NISHIDA Human ecology (Kyoritsu-Shuppan, Tokyo 1974).

NISHIDA, T.: Population-specific habits among wild chimpanzees. Jinnuigaku Zasshi. J. anthrop. Soc. Nippon 87: 196-197 (1979).

O'HERNE, L.: A short introduction to the geology of Surinam. Geologisch Mijnbouwkundige Dienst, Ministerie van Opbouw, Paramaribo, Surinam (1966).

OPPENHEIMER, J.R.: Behavior and ecology of the white-faced monkey, Cebus capucinus, on Barro Colorado Island. Unpublished doctoral dissertation, University of Ilinois \{1968\}.

PUSEY, A.E.: The physical and social development of wild adolescent chimpanzees ( $P a n$ troglodytes schweinfurthii). Diss. Abstr. Int. B38: $5791-5792$ (1978).

RASMUSSEN, D.R.: Evolutionary, proximate, and functional primate social ecology; in BATESON and KLOPFER Perspectives in ethology, vol. IV. Advantages of diversity (New York and London, 1981).

REYNOLDS, V. and REYNOLDS, F: Chimpanzees of the Budongo forest; in DEVORE Primate behavior (Holt, Rinehart \& Winston, New York 1965).

RICHARD, A.: A comparative study of the activity patterns and behavior of Alouatta villo$s a$ and Ateles geoffroyi. Folia primat. 12:24l-263 (1970).

RICHARDS, PW.: The tropical rain torest (Cambridge University Press, Cambridge 1966).

RODMAN, P.S.: Diets, densities and distribution of Bornean primates; in MONTGOMERY The ecology of arboreal folivores (Smithsonian Institution Press, Washington, D.C. 1978).

RONDINEL.LI, R. and KLEIN, L.L.: An analysis of adult social spacing tendencies and related social interactions in a colony of spider monkeys, Ateles geoffroyi, at the San Francisco Zoo. Folia primat. 25: $122-142$ (1976).

ROOSMALEN, M.G.M. VAN: Surinaams vruchtenboek, vol. 1 and 2, 555 pp. (University of Utrecht Press, Utrecht 1977). 
ROOSMALEN, M.G.M. VAN: Habitat preferences, diet, feeding strategy and social organisation of the black spider monkey (Ateles paniscus paniscus LINNAEUS 1758) in Surinam. Unpublished doctoral dissertation, Agricultural University of Wageningen (1980).

ROOSMALEN, M.G.M. VAN: Daily activity patterns, ranging behavior and food distribution in the black spider monkey, Ateles paniscus paniscus, in Surinam (in preparation).

ROOSMALEN, M.G.M. VAN: Polyspecitic associations between monkeys and terrestrial birds in the Surinam rain forest, with special reference to Ateles paniscus, Crax alector and Psophia crepitans (in preparation).

ROOSMALEN, M.G.M VAN.: Fruits of the Guianan Flora, 525 pp.(Veenman, Wageningem, 1985).

ROOSMALEN, M.G.M. VAN; LOTH, P, and KLEIN, L.L.: The spider monkeys, genus Ateles; in COMMBRA.FIL.HO and MITTERMEIFR Ecology and behaviour of Neotropical primates, vol. 2 (Rio de Janeiro, Brazilian Academy of Sciences, in press).

ROOSMALEN, M.G.M. VAN, MTTTERMEIER, R.A., and MILTON, K.: The bearded sakis, genus Chiropotes; in COIMBRA-FILHO and MTTERMEIER Ecology and behavior of Neotropical primates, vol. 1 (Rio de Janeiro, Brazilian Academy of Sciences, 1981).

ROSSAN, R.N. and BAERG, D.C.: Laboratory and feral hybridization of Ateles geoffroyi panamensis KELLOGG and GOLDMAN 1944 and A. fusciceps robustus ALLEN 1914 in Panama. Primates 18: 235-237 (1977).

SABATER PI, J.: Feeding behavior and diet of chimpanzees (Pan troglodytes) in the Okorobiko Mountains of Fio Muni (West Africa). Zeitschr. f. Tierpsych. 50: 265-281 (1979).

SANDERSON, I.T.: A joumey in Dutch Guyana. Geogr. Journ. 93(6): 468-490 (1939).

SANDERSON, I.T: A brief review of the mammals of Suriname (Dutch Guyana), based upon a collection made in 1938. Proc. zool. Soc., London 119: 755-789 (1949).

SANDERSON, IT: The monkey kingdom. Doubleday, Garden City, New York (1957).

SCHOENER, TW: Theory of feeding strategies. Ann. Rev. ecol. Syst. 2: $369-404$ (1971).

SCHULZ, J.P. Ecological studies on rain forest in northern Suriname; in HULSTER and LANJOUW The vegetation of Suriname, vol. 2. pp. 1-267 (1960).

SCOTT, H.J., Ir.; STRUHSAKER, T.T.; GLANDER, K., and CHIRIVI, H.: Primates and their babitats in northern Colombia, with recommendations for future management and research; in First interamerican conference on conservation and utilization of American non-human primates in biomedical research, pp. 30-50, Pan American Health Organization, Washington, D. C. (1976).

SKUTCH, A.F.: Live history of the Quetzal. Condor 46: 213-235 (1944).

SMITH, C.C.: The coevolution of plants and seed predators; in GILBERT and RAVEN Coevolution of animals and plants (University of Texas Press 1975).

SMYTHE, N.: Relationships between fruiting seasons and seed dispersal methods in a Neotropical torest. Amer. Natur. 104: 25-35 (1970).

SNOW, B.K.: Notes on the behavior of three Cotingidae. The Auk 78: 150-16] (1961).

SNOW, B.K.: A field study of the bearbed bellbird in Trinidad. Ibis 112:299-329 (1970).

SNOW, DW.: The natural history of the oilbird.1, General behavior and breeding habits. Zoologica 46: $27-48$ (1961).

SNOW, DW. A field study of the black and white manakin, Manacus manacus, in Trinidad. 7oologica 47: 65-104 (1962a).

SNOW, D.W. A tield study of the golden-headed manakin, Pipra erythrocephala, in Trinidad. Zoologica 47: 183-198 (1962b). 
SNOW, DW.: The natural history of the oilbird, Steatomis canpensis, in Trinidad. W. I. II Bopilation, breeding ecology and food. Zoologica 47: 199-221 (1962c).

SNOW, D.W: A possible selective factor in the evolution of fruiting seasons in tropical forest. Oikos 15: 274-281 (1965).

SNOW, DW.: Evolutionary aspects of truit-eating by birds. Ibis 113: 194202 (1971).

SNOW, B.K. and SNOW, DW: The feeding ecology of tanagers and honeycreepers in Trinidad. The Auk 88: 291-322 (1971)

STAAI, F. VAN DER.: Jacht in Bigi Poika. Mimeographed report, Agncultural Uniwersity of Wageningen, Celos, Paramaribo, Suriname (1975)

STRUHSAKER, TT.: Correlates of ecology and social organization among African cercopithecines. Folia primat. 11: 80-118 (1969).

STRUHSAKER, TT.; GLANDER, K.; CHIRIVI, H., and SCOTT, N.J.: A survey of primates and their habitats in northern Colornbia; in Primate censusing studies in Peru and Colombia, pp. 43-78, Pan American Heaith Organization (1975). 


\section{This page intentionally left blank}




\section{Appendix}

Distribution and density of main tood plant and sleeping tree species in the combined core area of two leading-female spider monkeys belonging to the Voltzberg study group, whose ranging behavior was best studied. For plotting purposes, the area was subdivided in rectangular $1 / 2$-hectare blocks. Shaded areas indicate non-suitable habitat (e.g., open granite, liane forest) that was not sampled. Both botanical and vernacular names (when available) are given, while the total number of individual plants plotted in the area is given in parentheses. Few species ( $n^{\circ} 53$ and 119) were very abundant locally and consequently only part of its population was plotted. Of several species, in particular lianes, only the individual plants actually seen exploited by the monkeys were plotted (n. 25, 26, $27,93,115$ and 116). For the purposes of this study, tagging and plotting was restricted to those individual plants, that obviously had reached "their" preferred stratum which, in general, implies the phenomenon of flowering and fruiting. Plants providing the monkeys only with edible flush leaves were plotted when reaching at least 12 meters in height, the lower limit of Ateles vertical range.

Besides mountain savanna forest with indicator species Guettarda acreana (114) and Ecclinusa guianensis (119), pina swamp forest with indicator species Euterpe oleracea, Pachira insignis (14), Eper. rua talcata (61) and Virola surinamensis (111), and high torest, a subtype of high forest on relatively red (ferrosiallitic) forest-soil may be distinguished, covering roughly blocks $A$ and $B$, showing relatively abundant leading species, such as Protium polybotryum (18), Tetragastris altissima (19), Tetragastris panamensis (20) and Capparis maroniensis (21). 


\section{This page intentionally left blank}


HOME RANGE SHOWING DISTRIBUTION AND DENSITY OF FOOD PLANTS

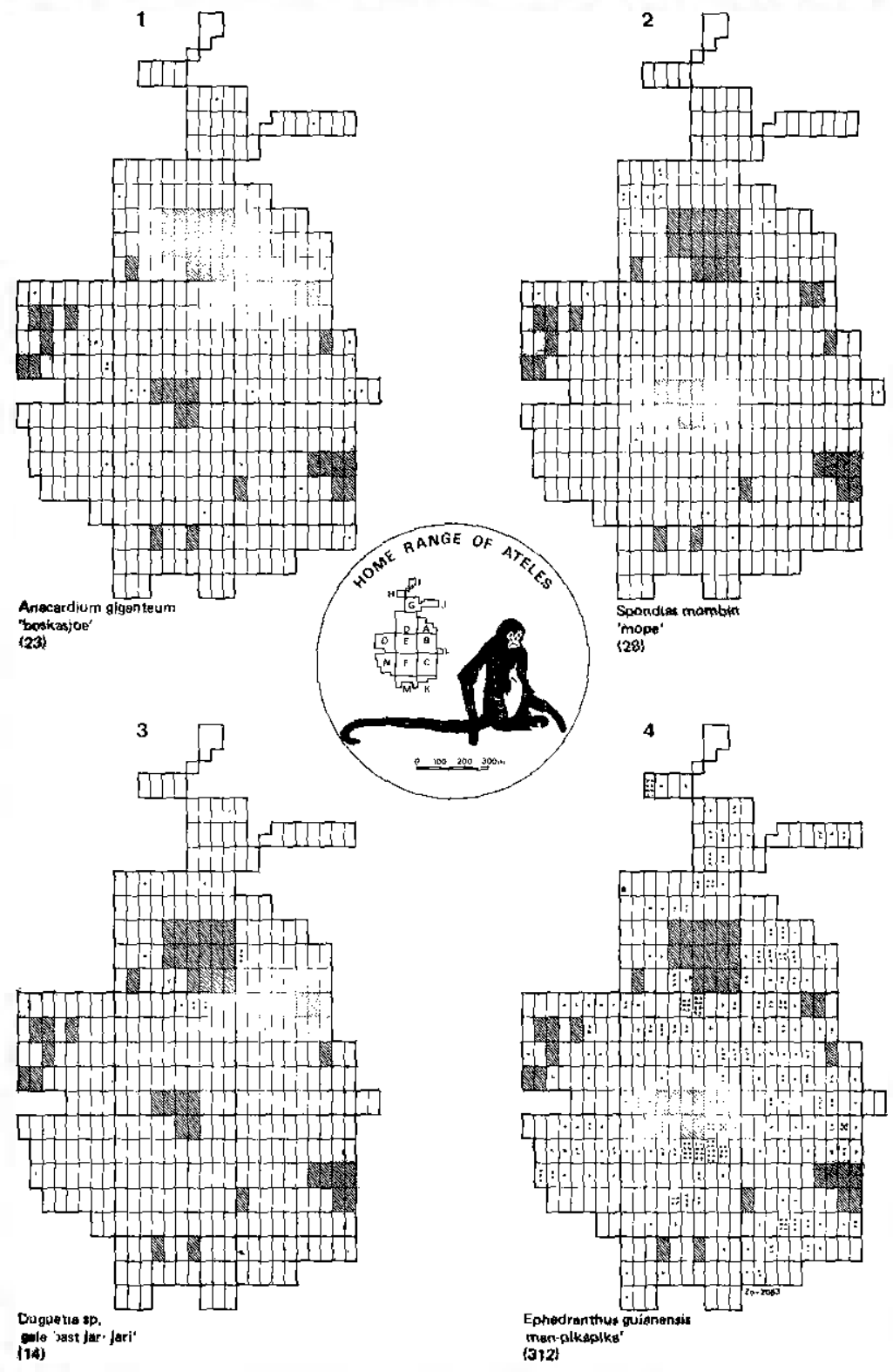


HOME RANGE SHOWING DISTAIBUTION AND DENSITY OF FOOD PLANTS

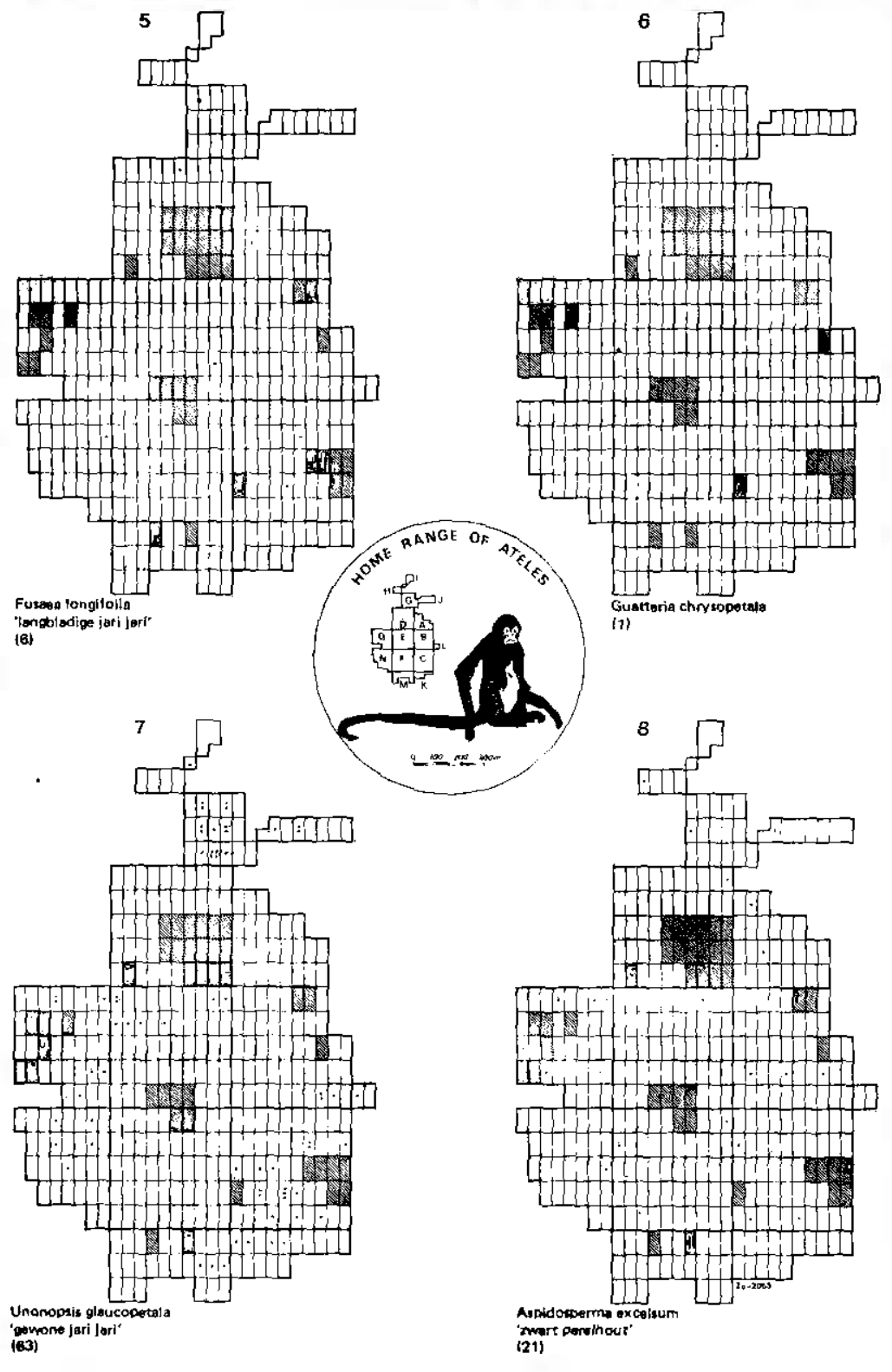


HOME RANGE SHOWING DISTRIBUTION AND DENSITY OF FOOD PLANTS

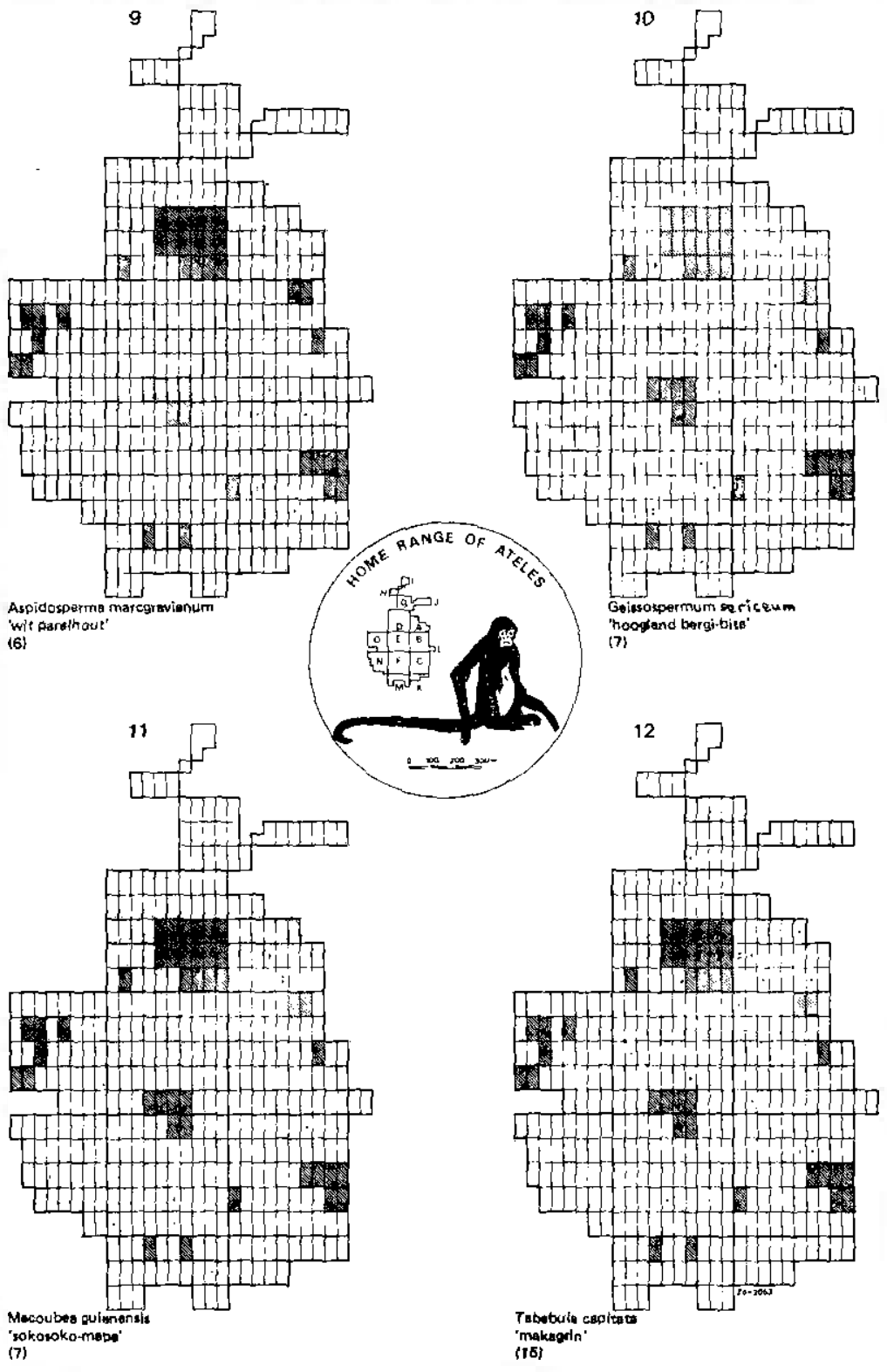


hOME RANGE SHOWING DISTRIBUTION AND DENSITY OF FOOD PLANTS

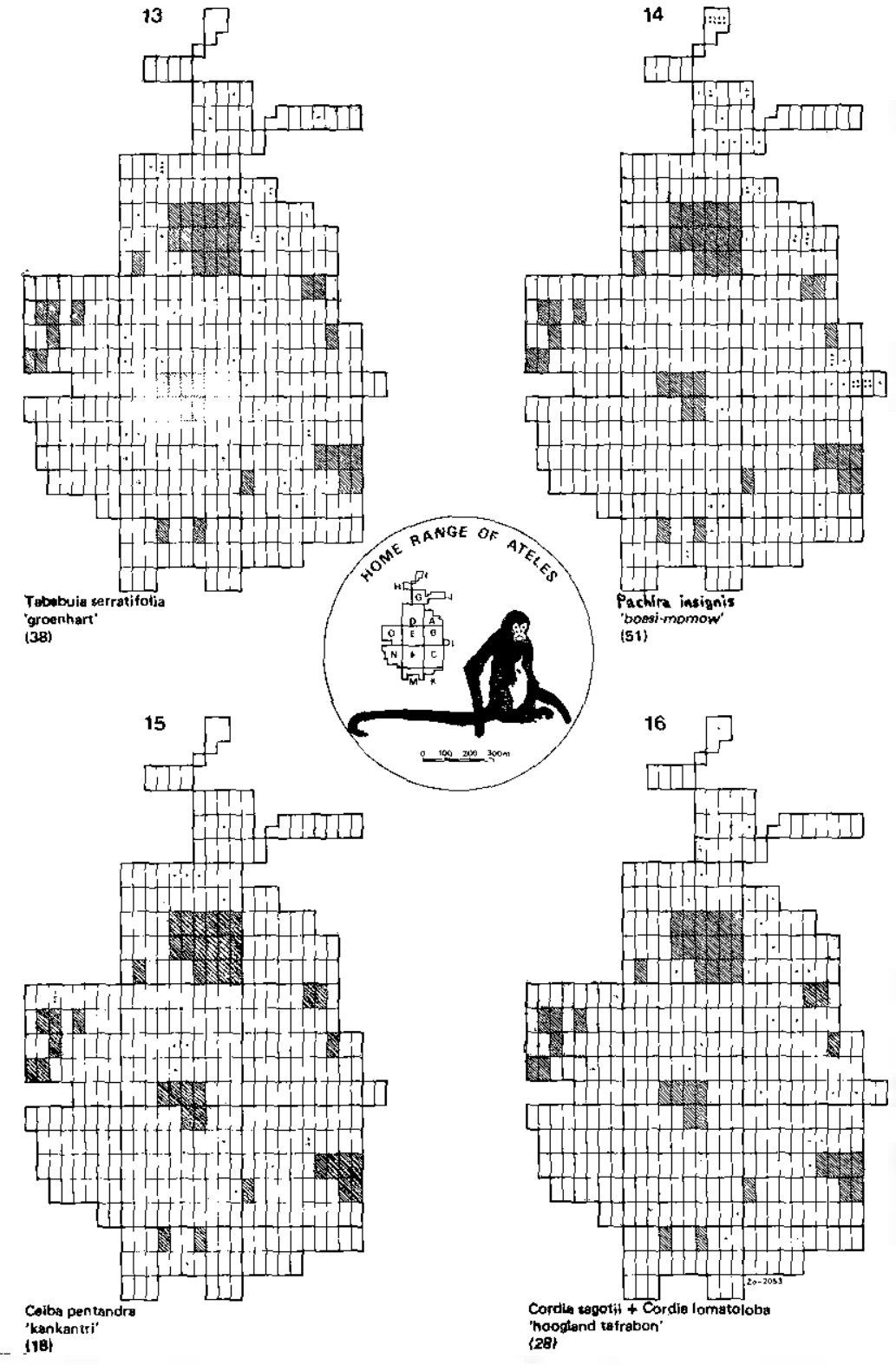


HOME RANGE SHOWING DISTRIBUTION AND DENSITY OF FOOD PLANTS

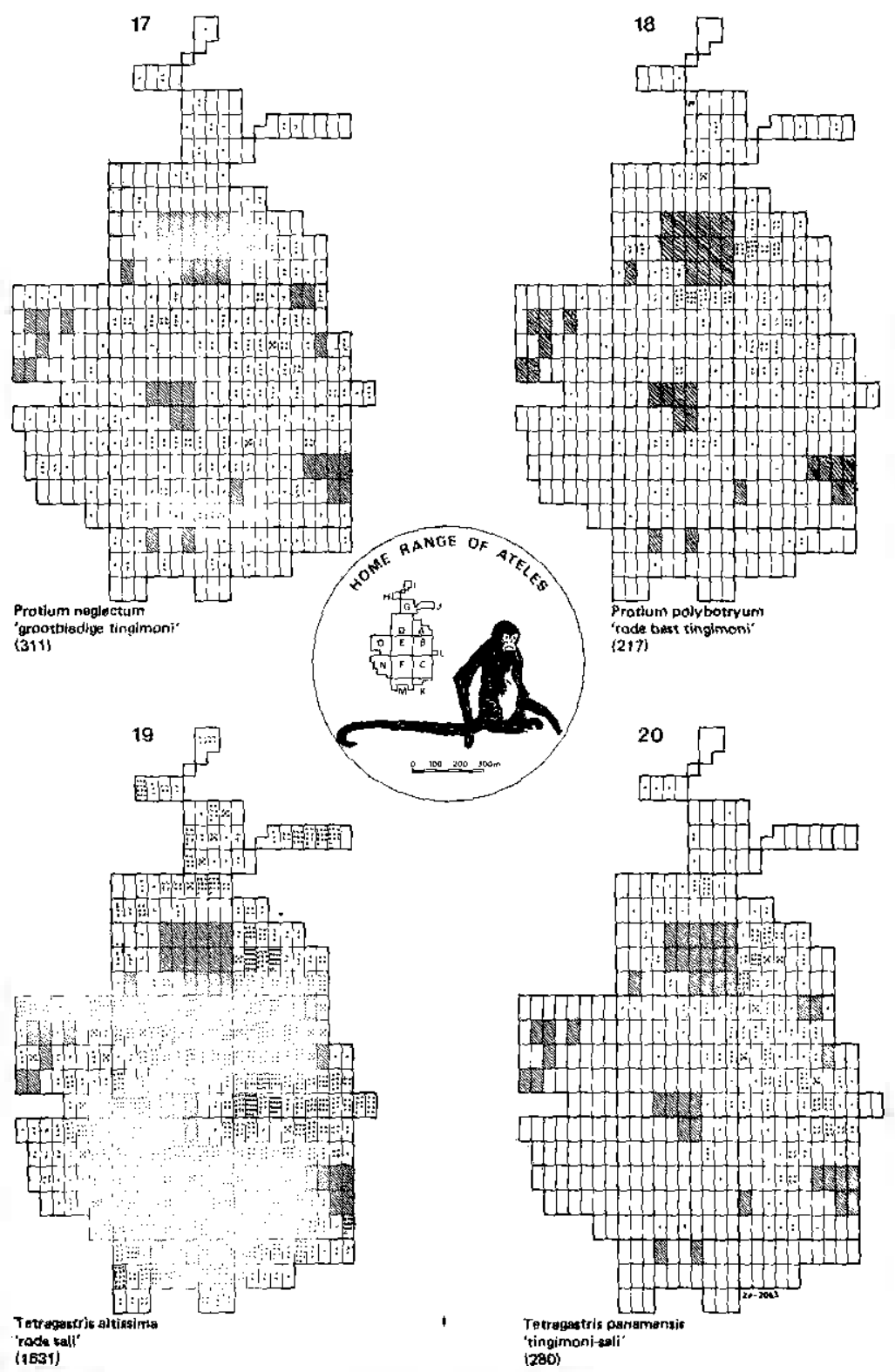

Habltat..- 
HOME RANGE SHOWING DISTRIBUTION AND DENSITY OF FOOD PLANTS

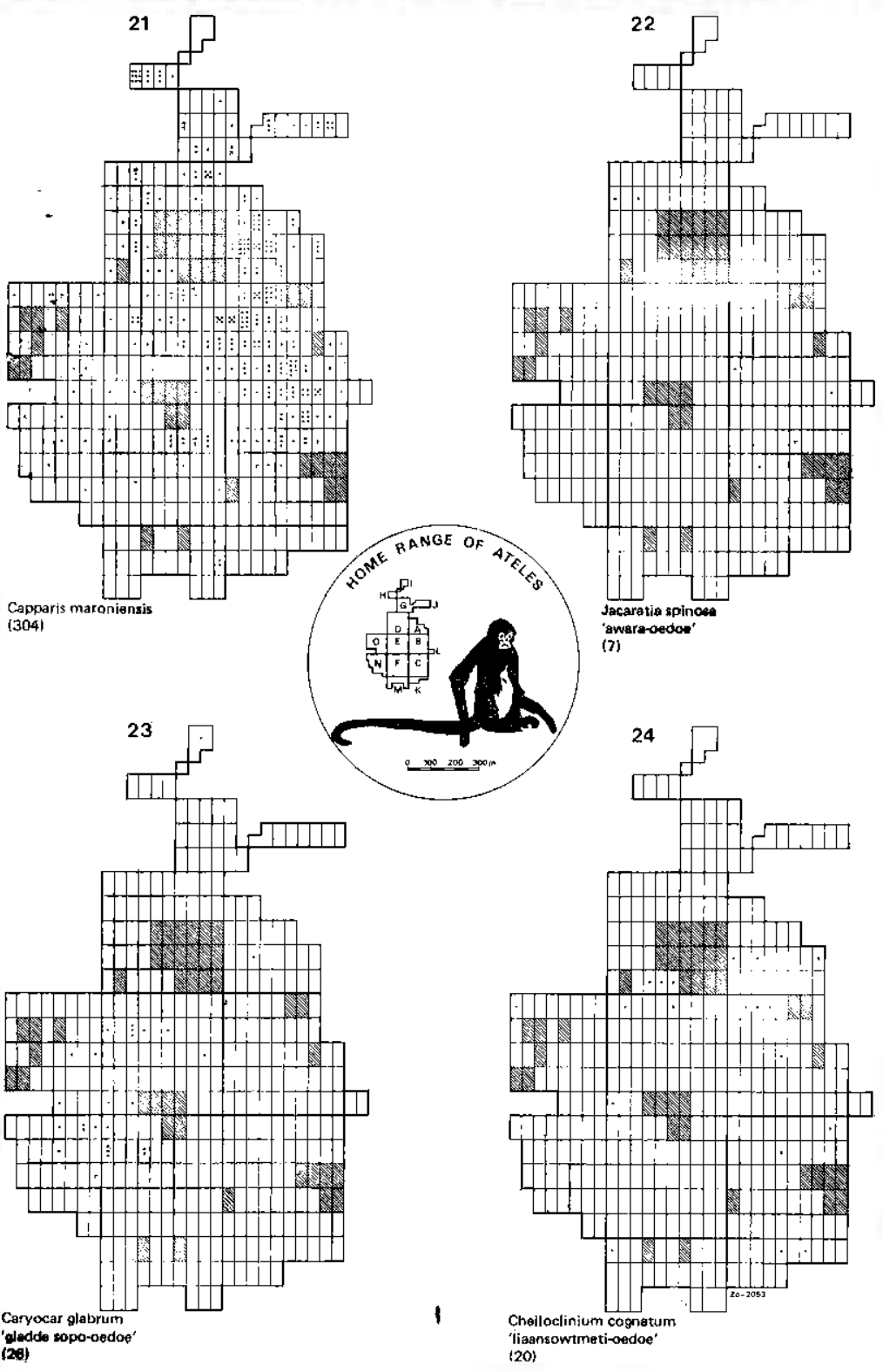


HOME RANGE SHOWING DISTRIBUTION AND DENSITY OF FOOD PLANTS

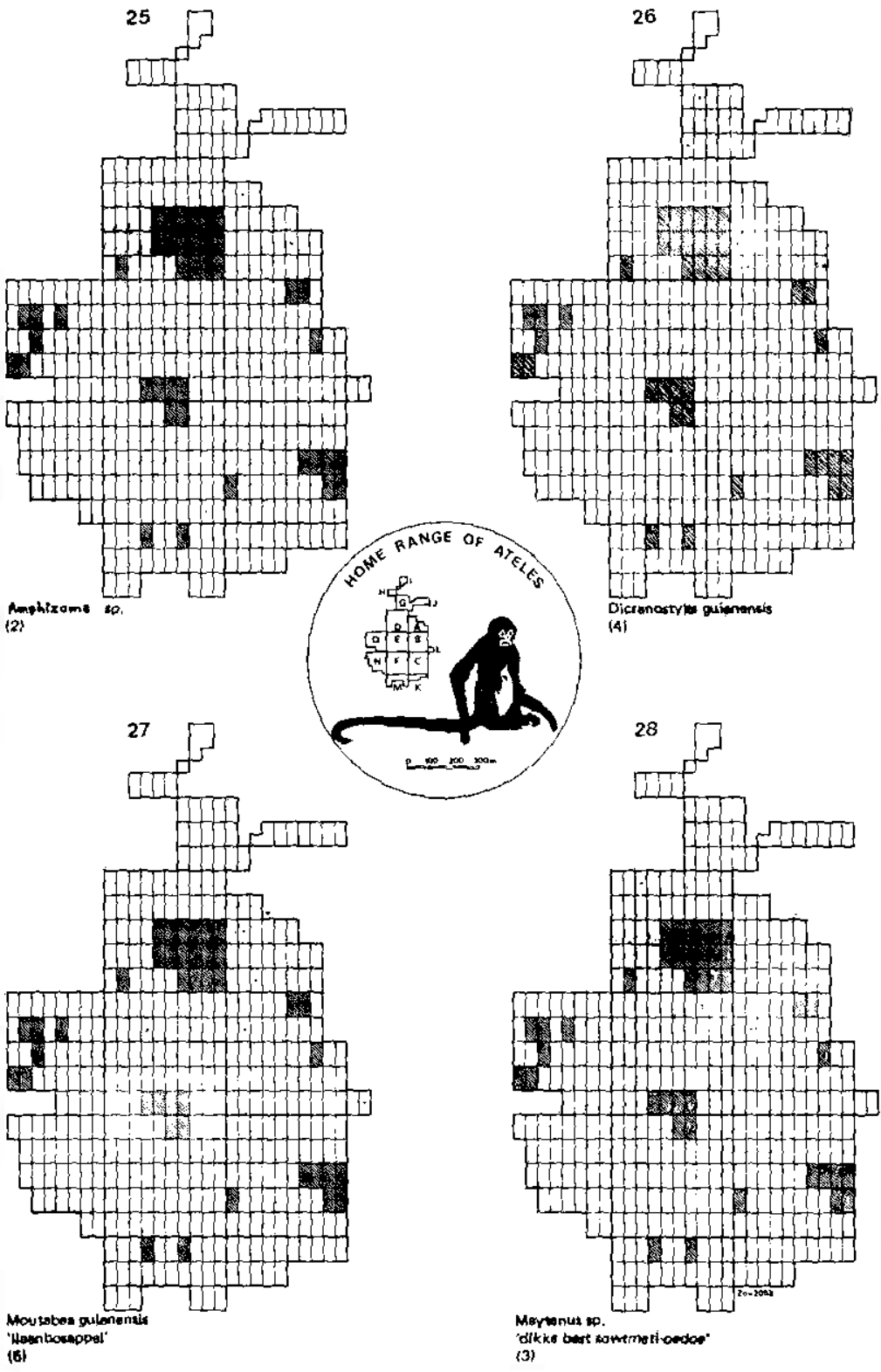


HOME RANGE SHOWING DISTRIBUTION AND DENSITY OF FOOD PLANTS

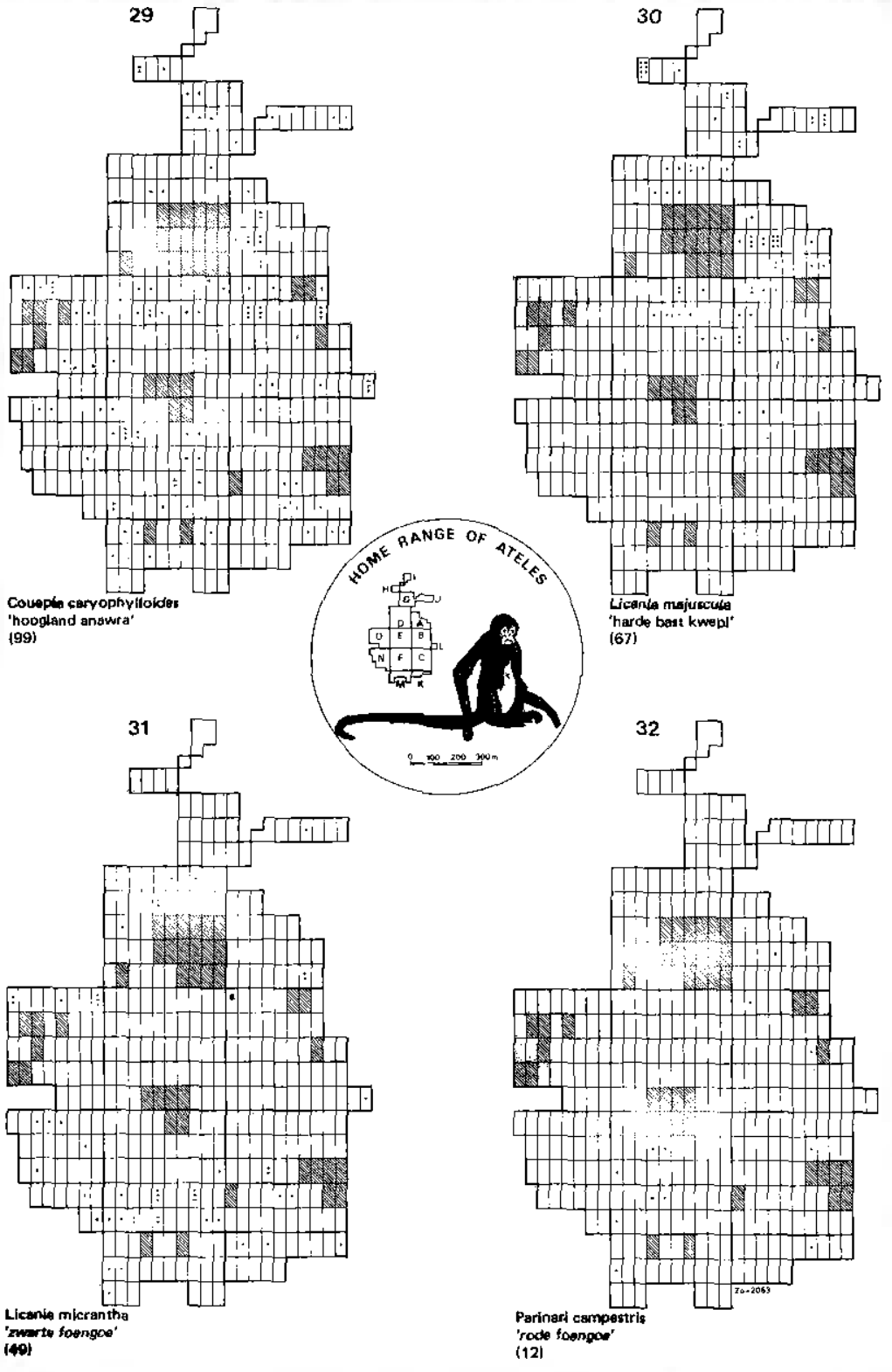


hOME RANGE SHOWING DISTRIBUTION AND DENSITY OF FOOD PLANTS

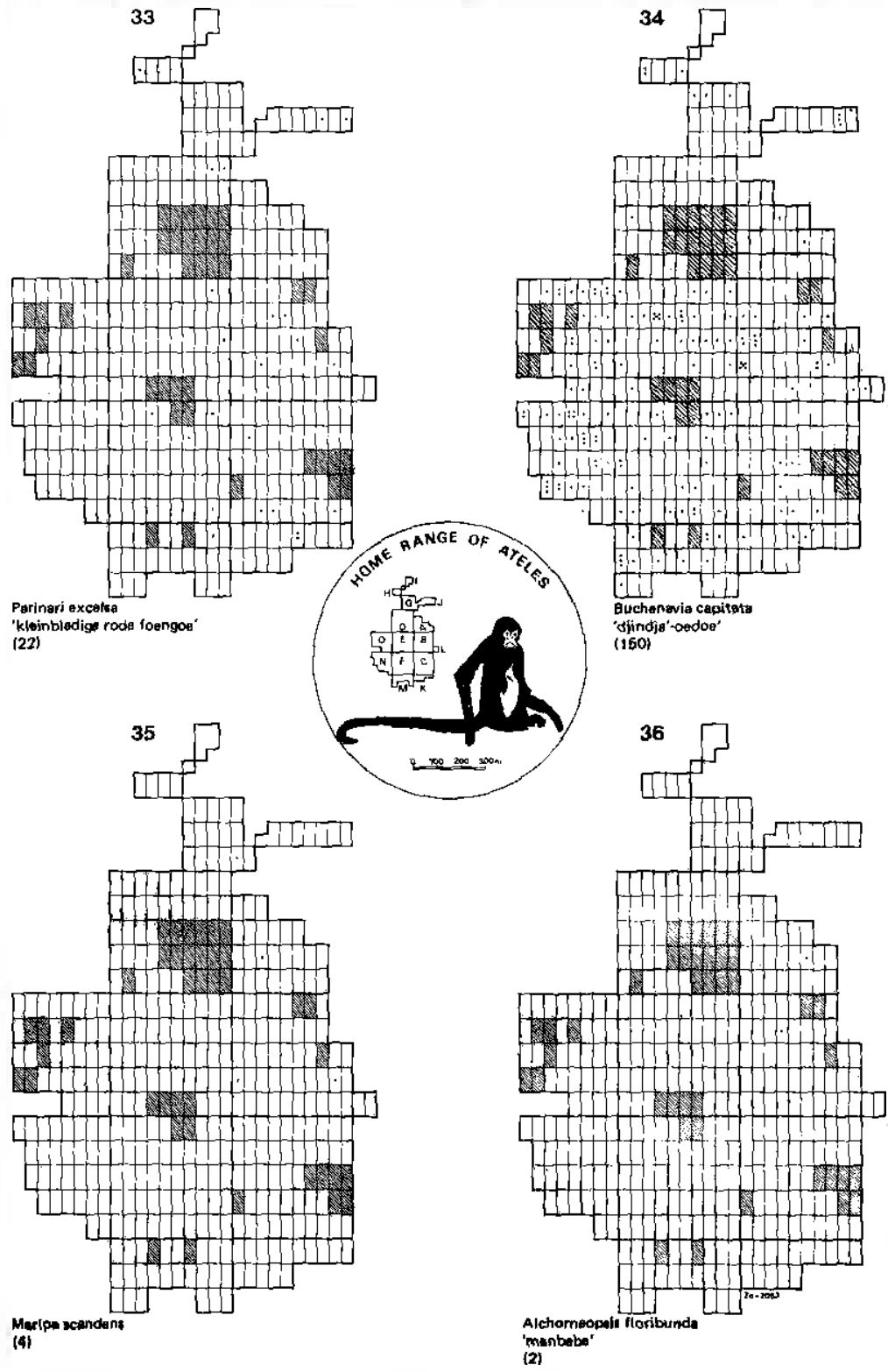

Habitat... 
HOME RANGE SHOWING DISTRIBUTION AND DENSITY OF FOOD PLANTS

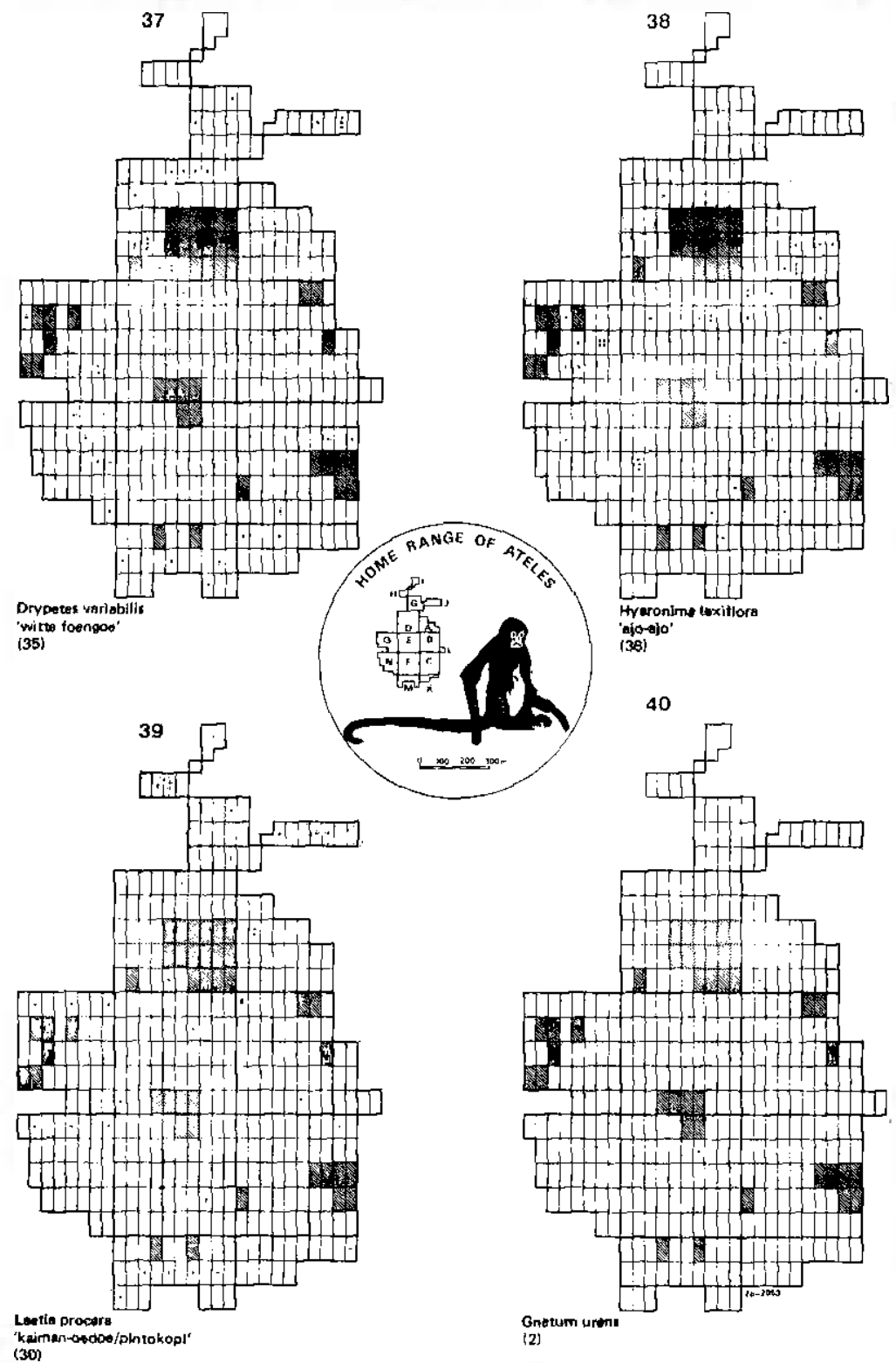


HOME RANGE SHOWING DISTRIBUTION AND DENSITY OF FOOD PLANTS

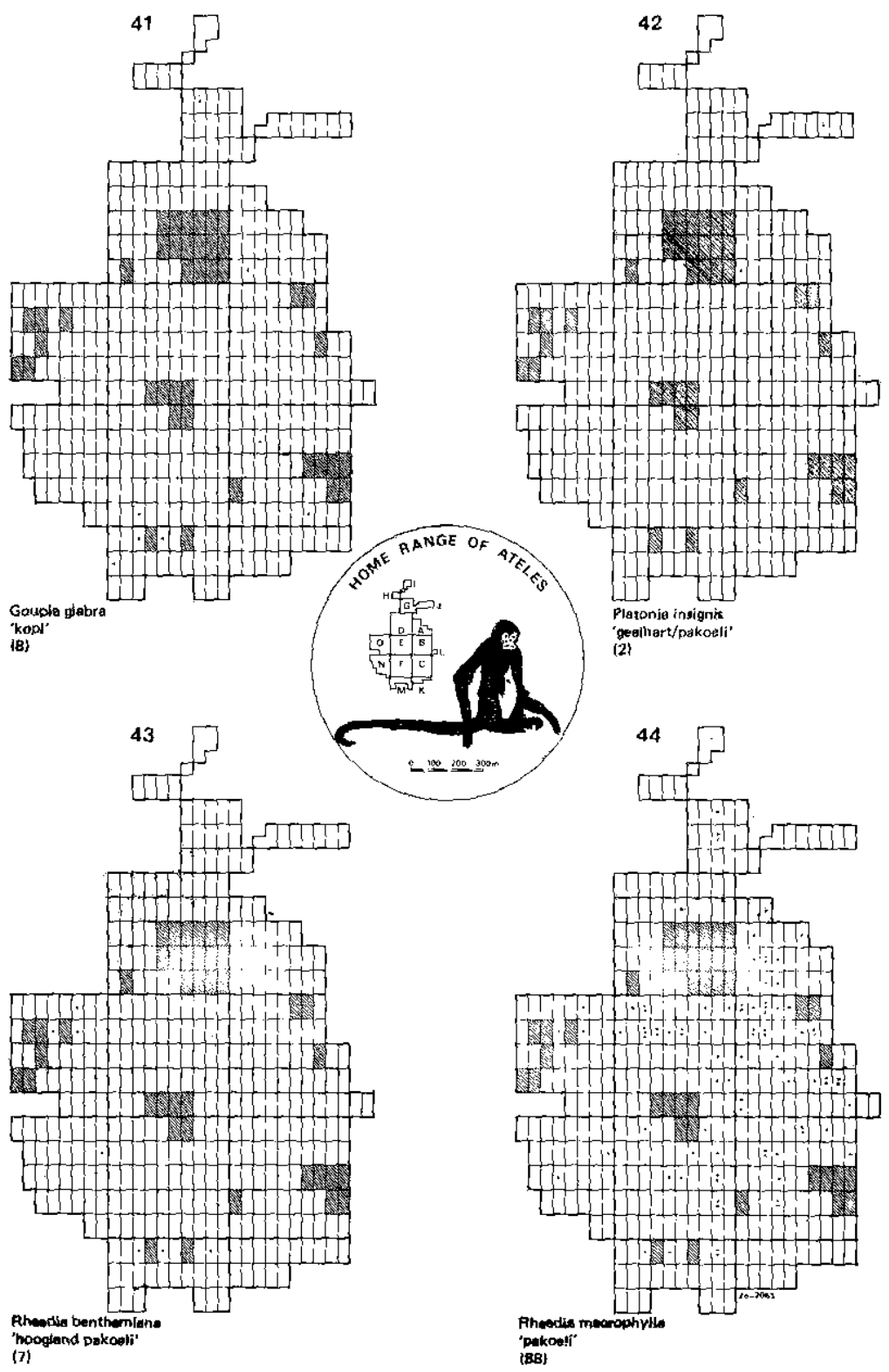


HOME RANGE SHOWING DISTRIBUTION AND DENSITY OF FOOD PLANTS

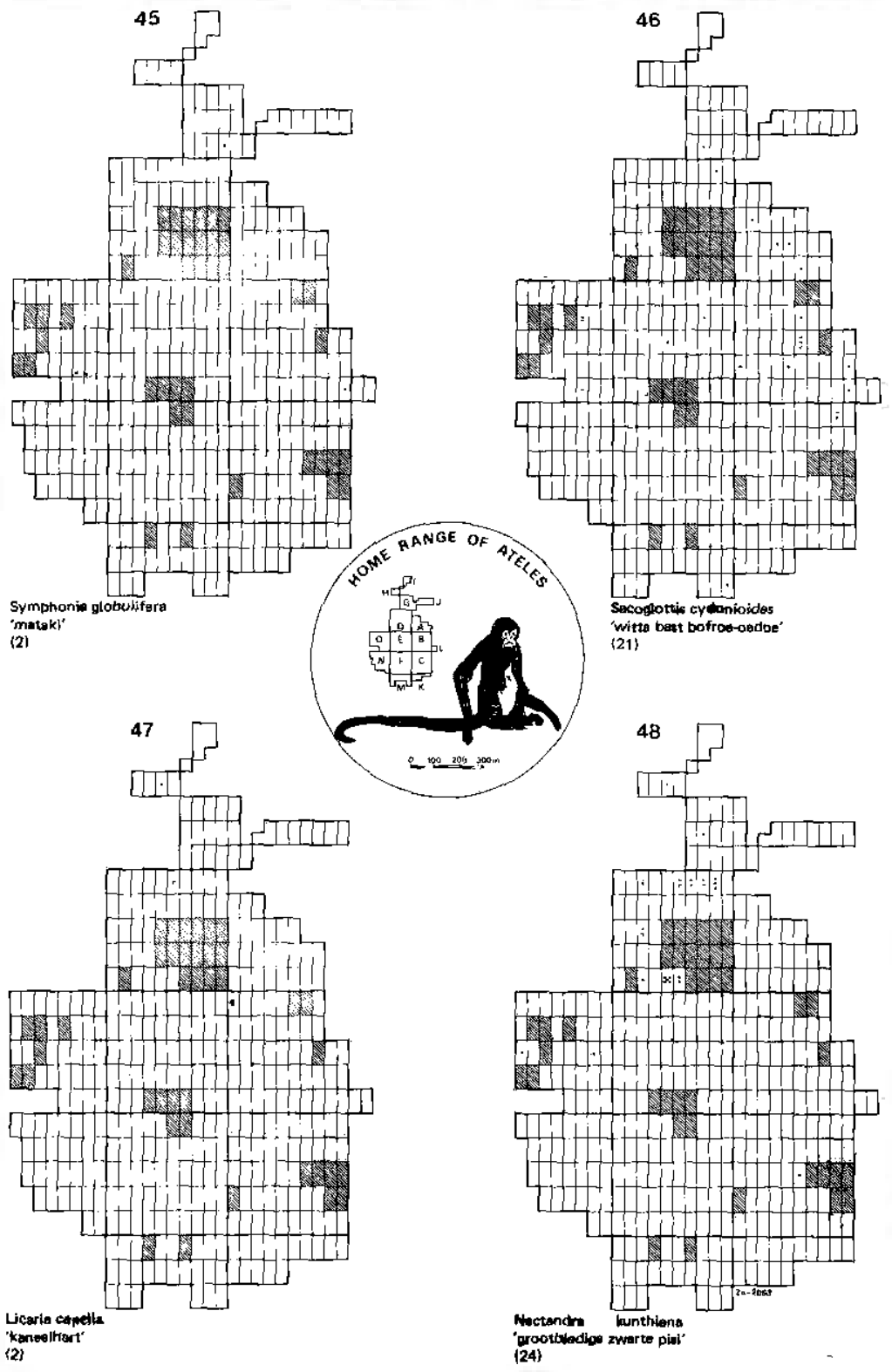


HOME RANGE SHOWING DISTRIBUTION AND DENSITY OF FOOD PLANTS

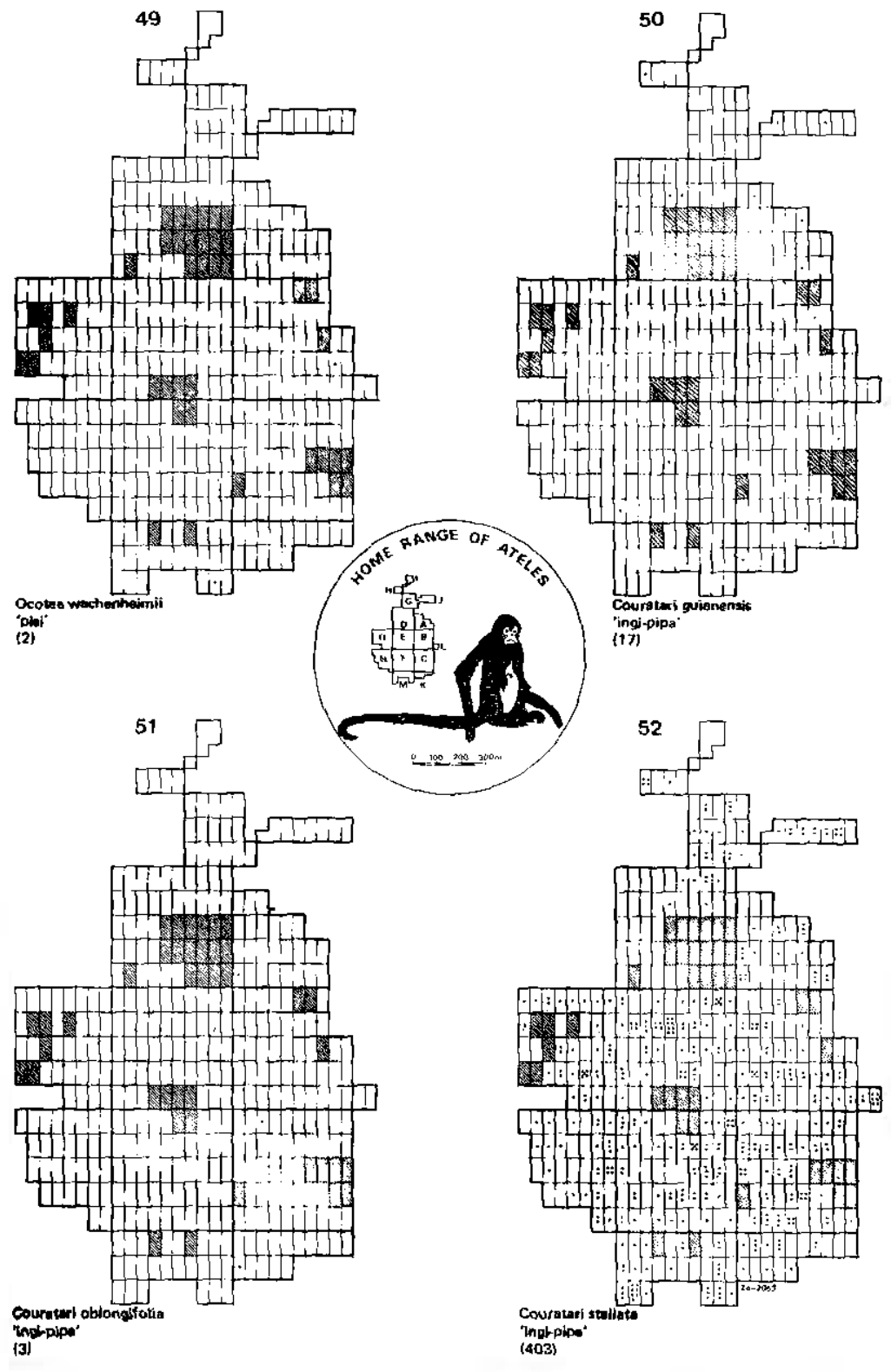

Habitat... 
HOME RANGE SHOWING DISTRIBUTION AND DENSITY OF FOOD PLANTS

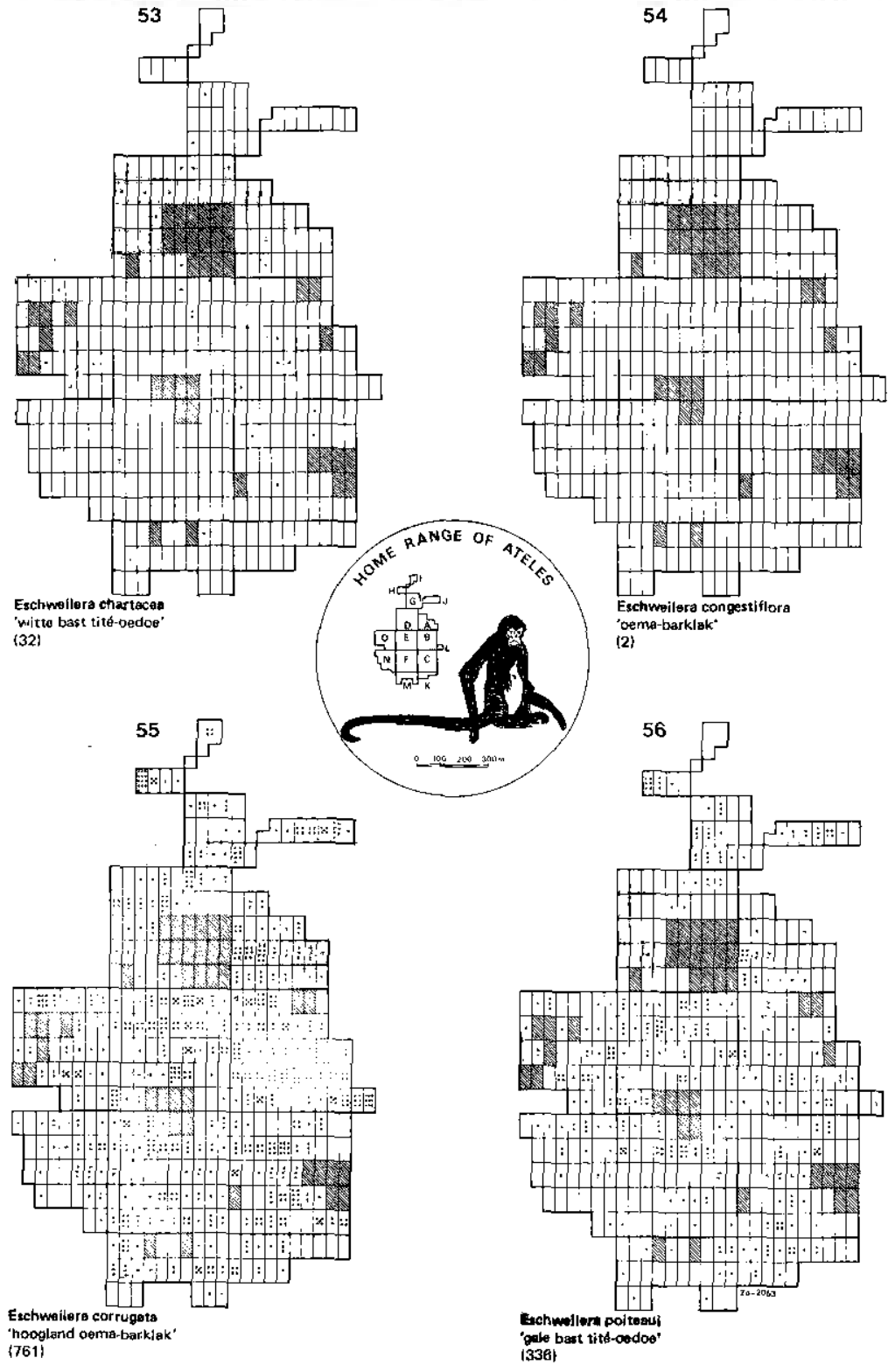


HOME RANGE SHOWING DISTRIBUTION AND DENSITY OF FOOD PLANTS

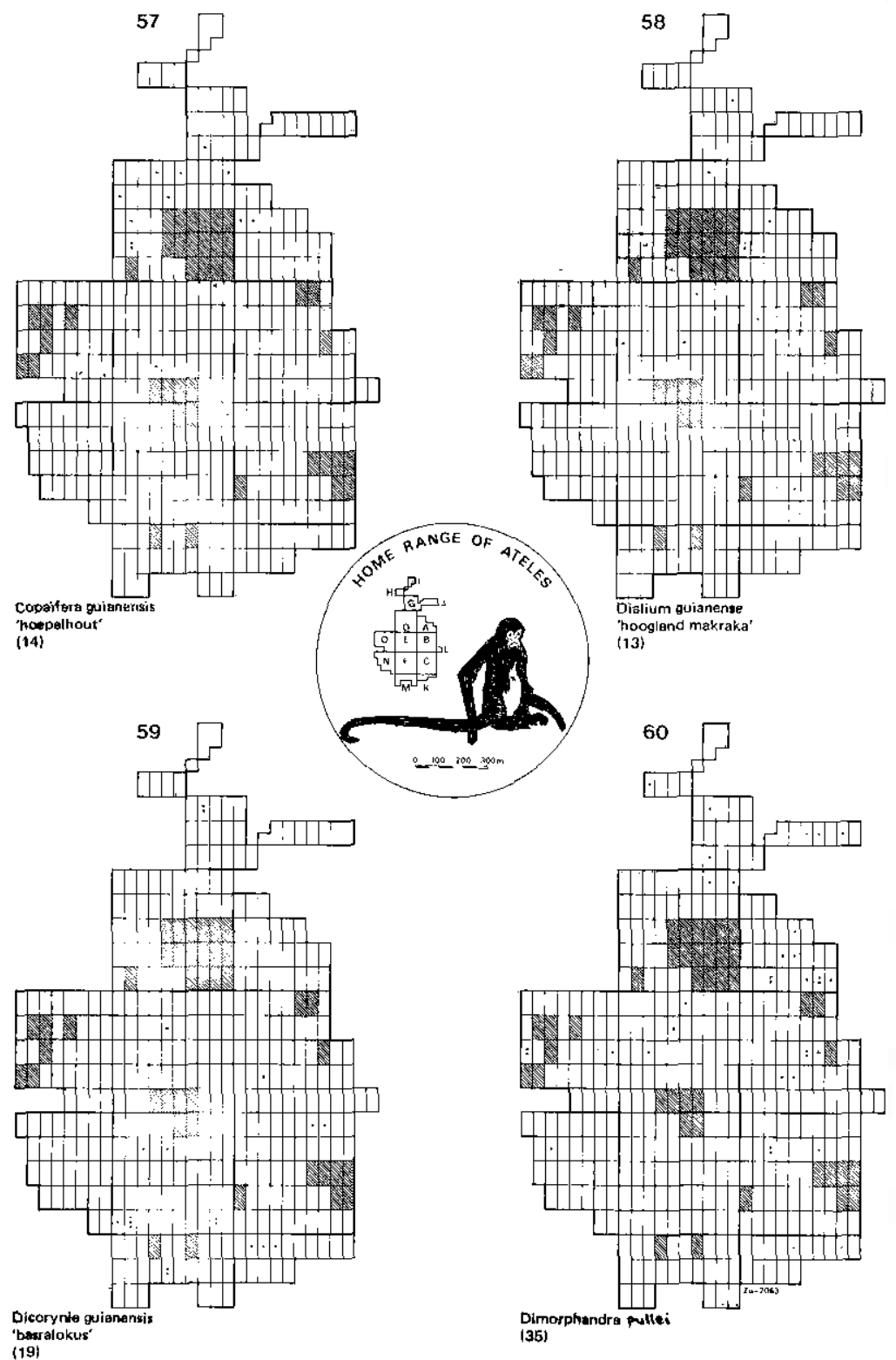

Habitat... 
HOME RANGE SHOWING DISTRIBUTION AND DENSITY OF FOOD PLANTS

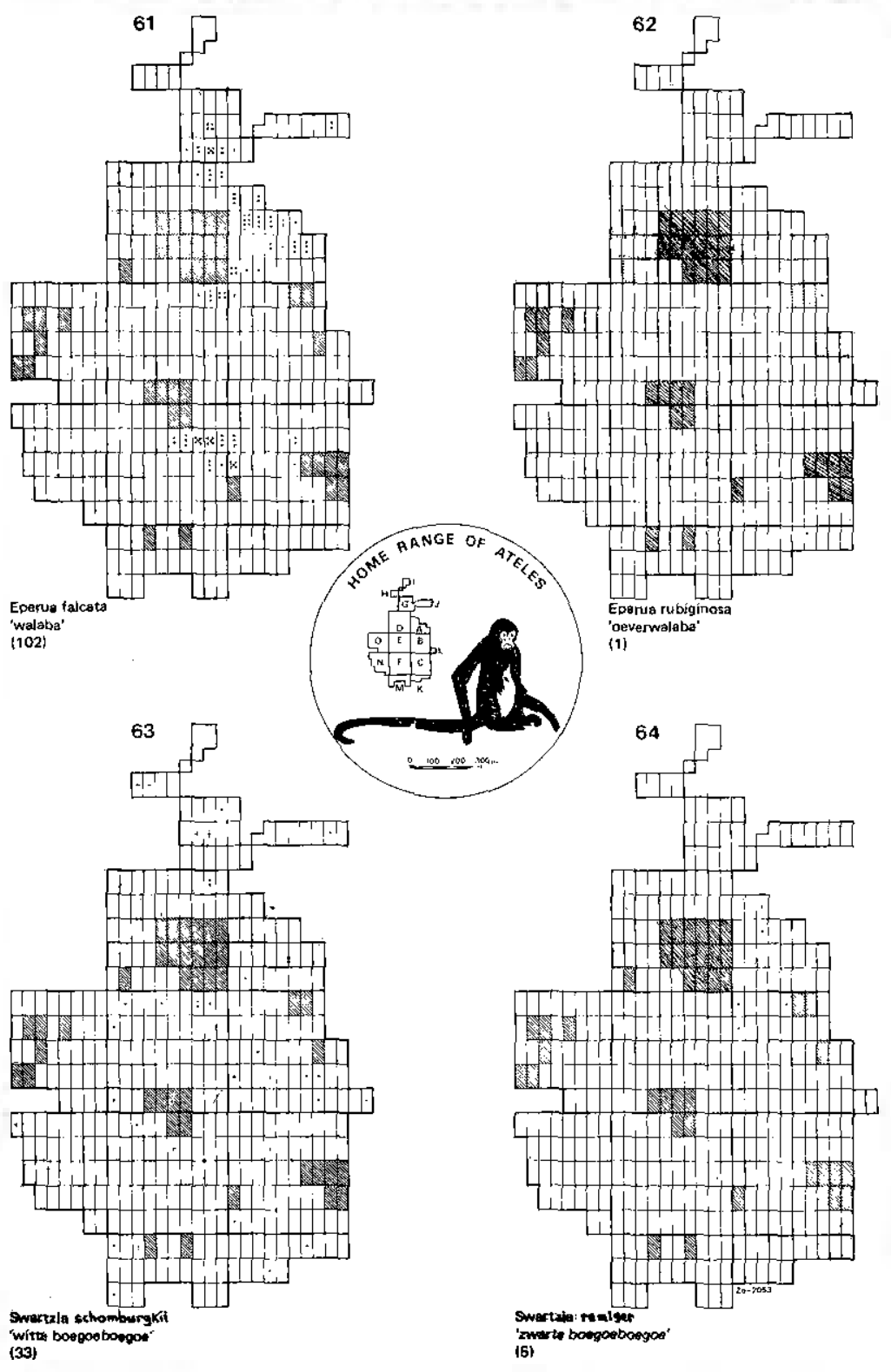


HOME RANGE SHOWING DISTRIBUTION AND DENSITY OF FOOD PLANTS

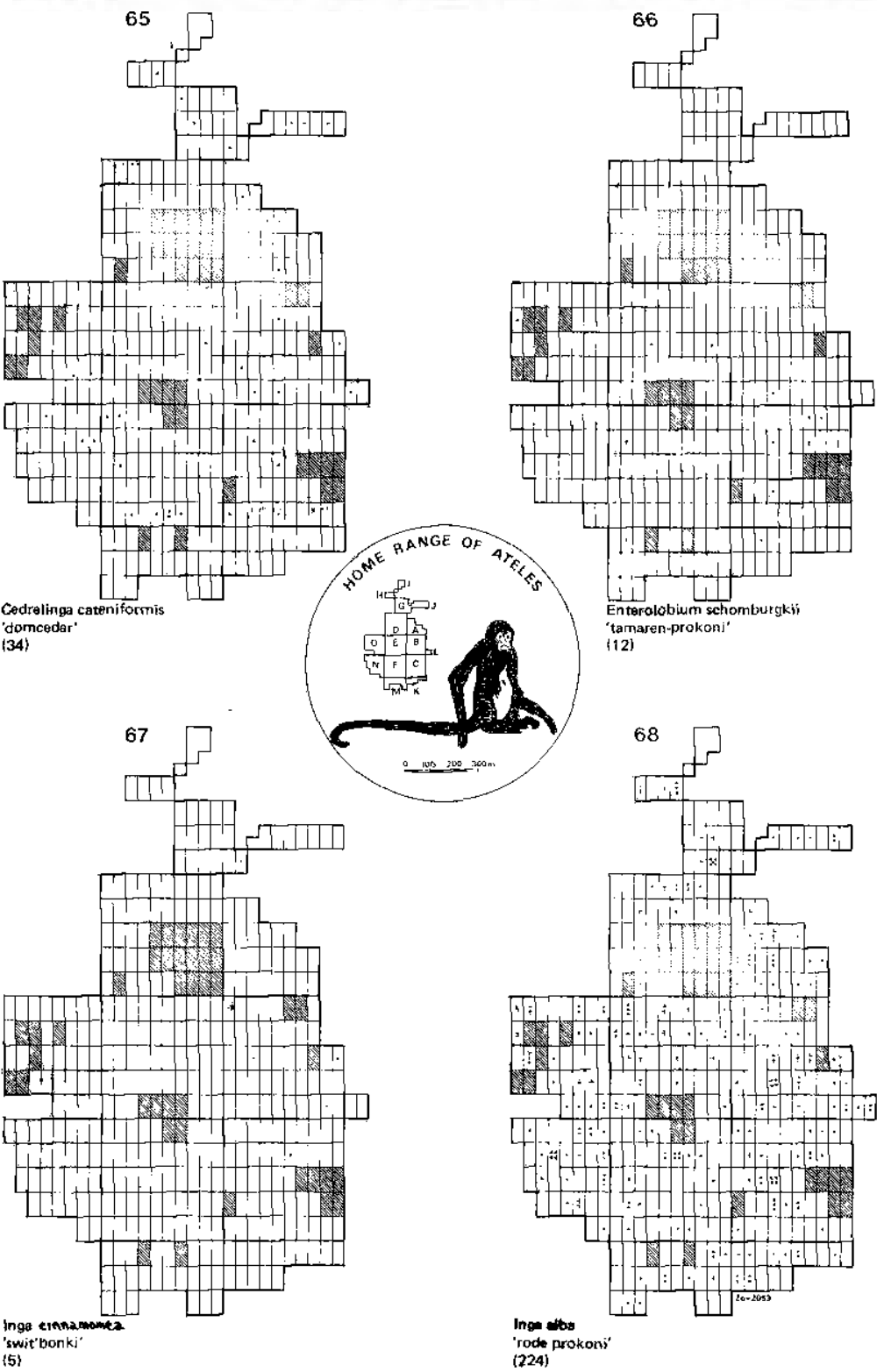


HOME RANGE SHOWING DISTRIBUTION AND DENSITY OF FOOD PLANTS

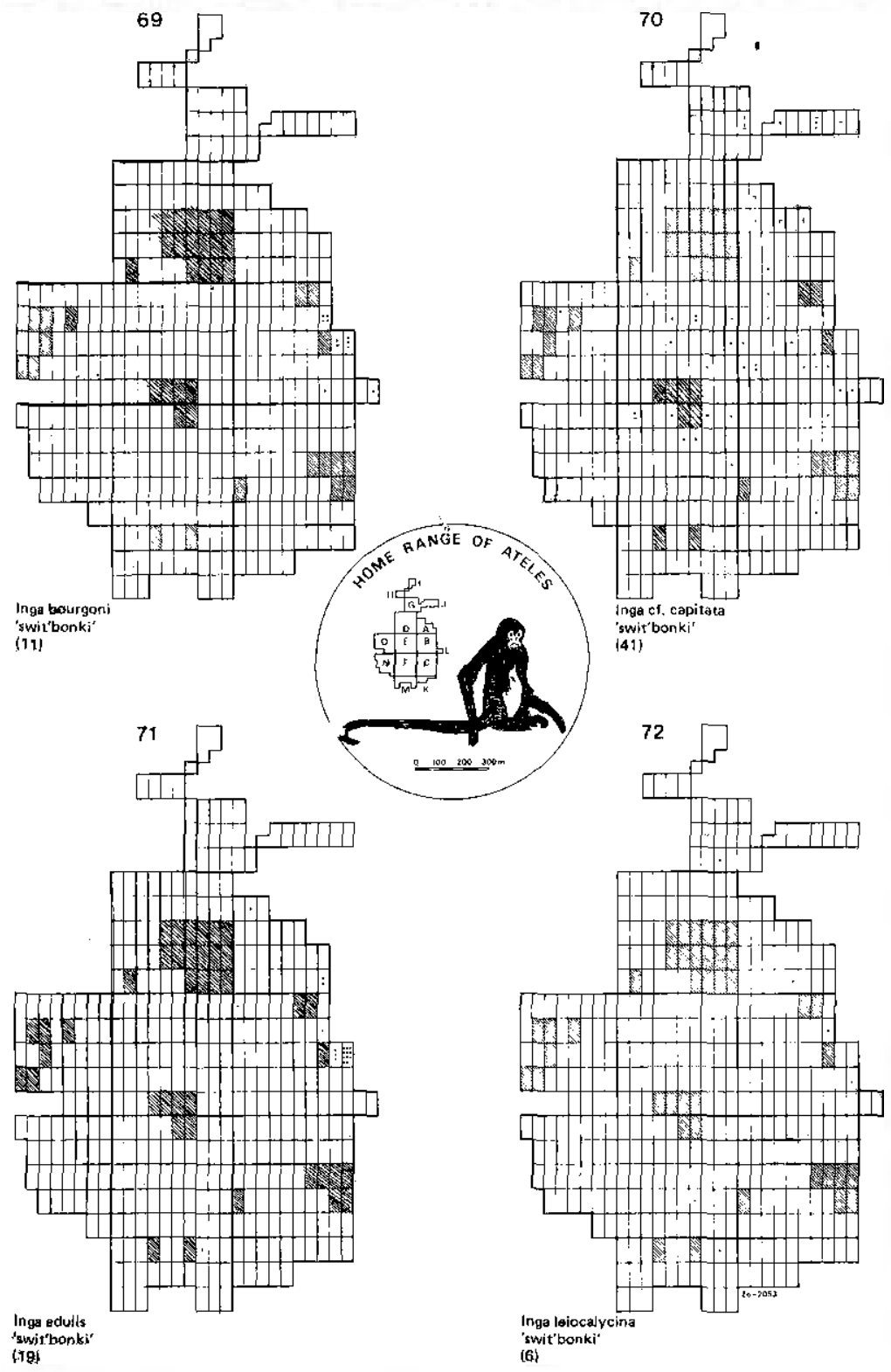


HOME RANGE SHOWING DISTRIBUTION AND DENSITY OF FOOD PLANTS

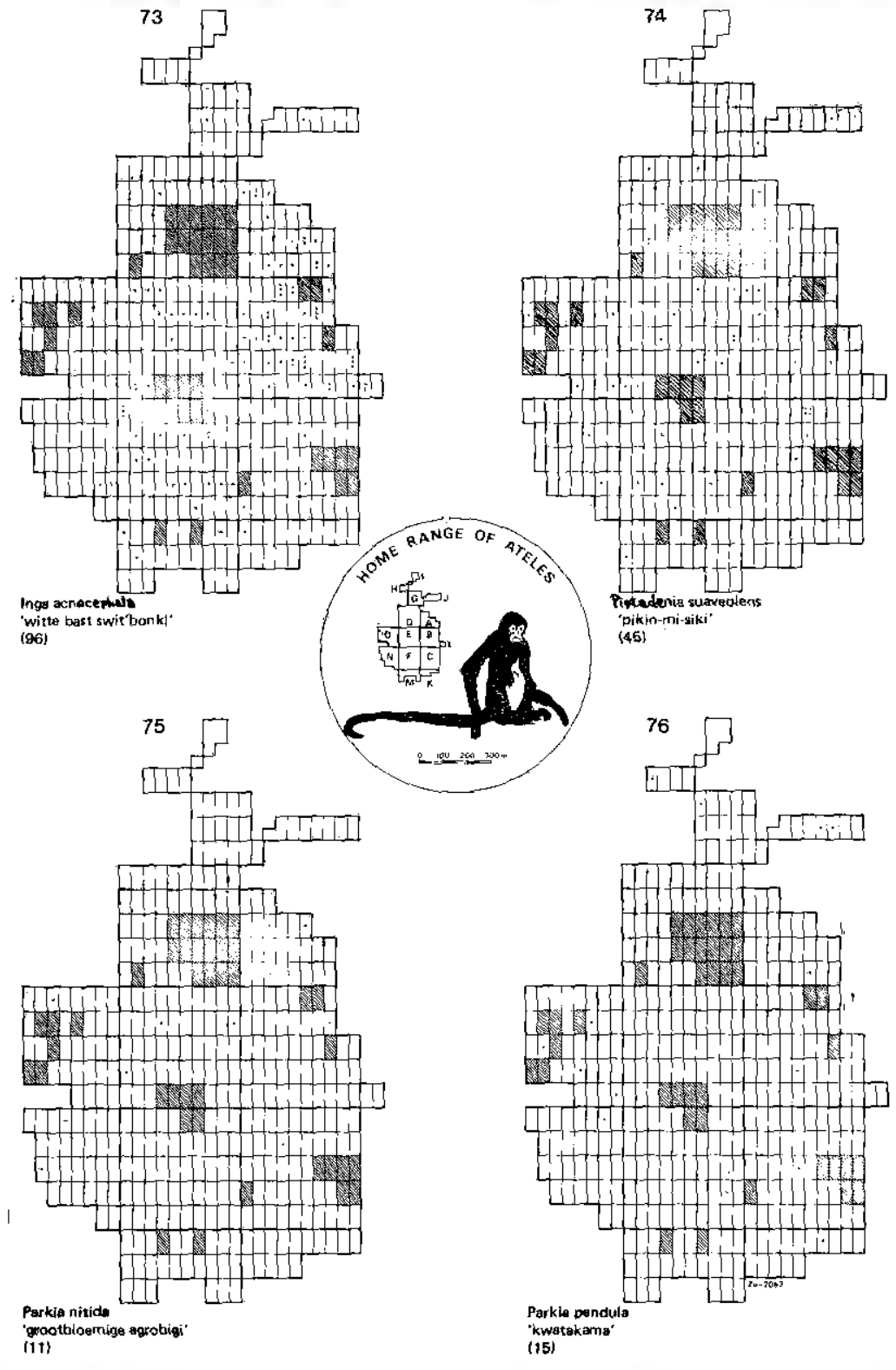


HOME RANGE SHOWING DISTRIBUTION AND DENSITY OF FOOD PLANTS

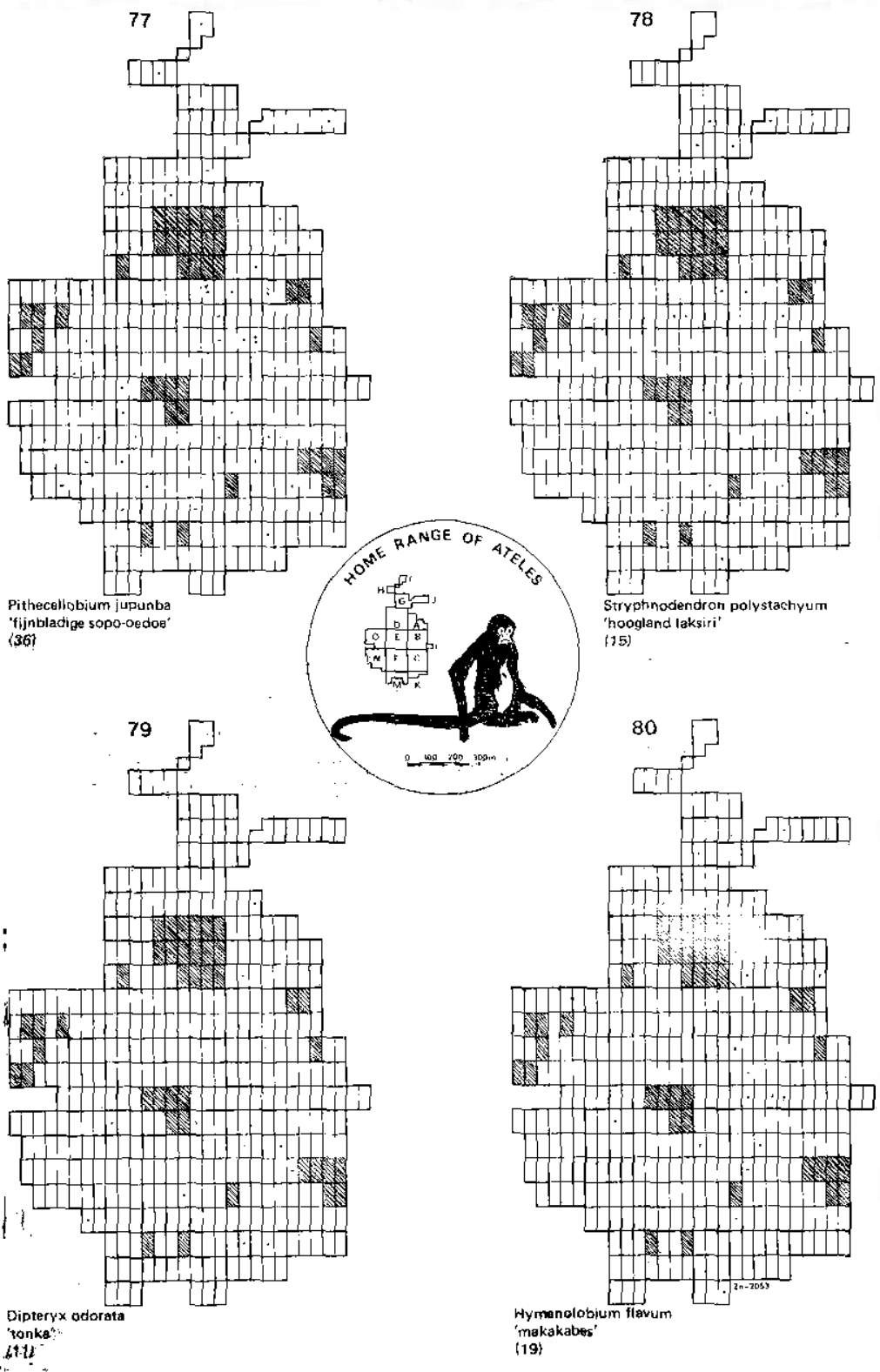


HOME RANGE SHOWING DISTRIBUTION AND DENSITY OF FOOD PLANTS

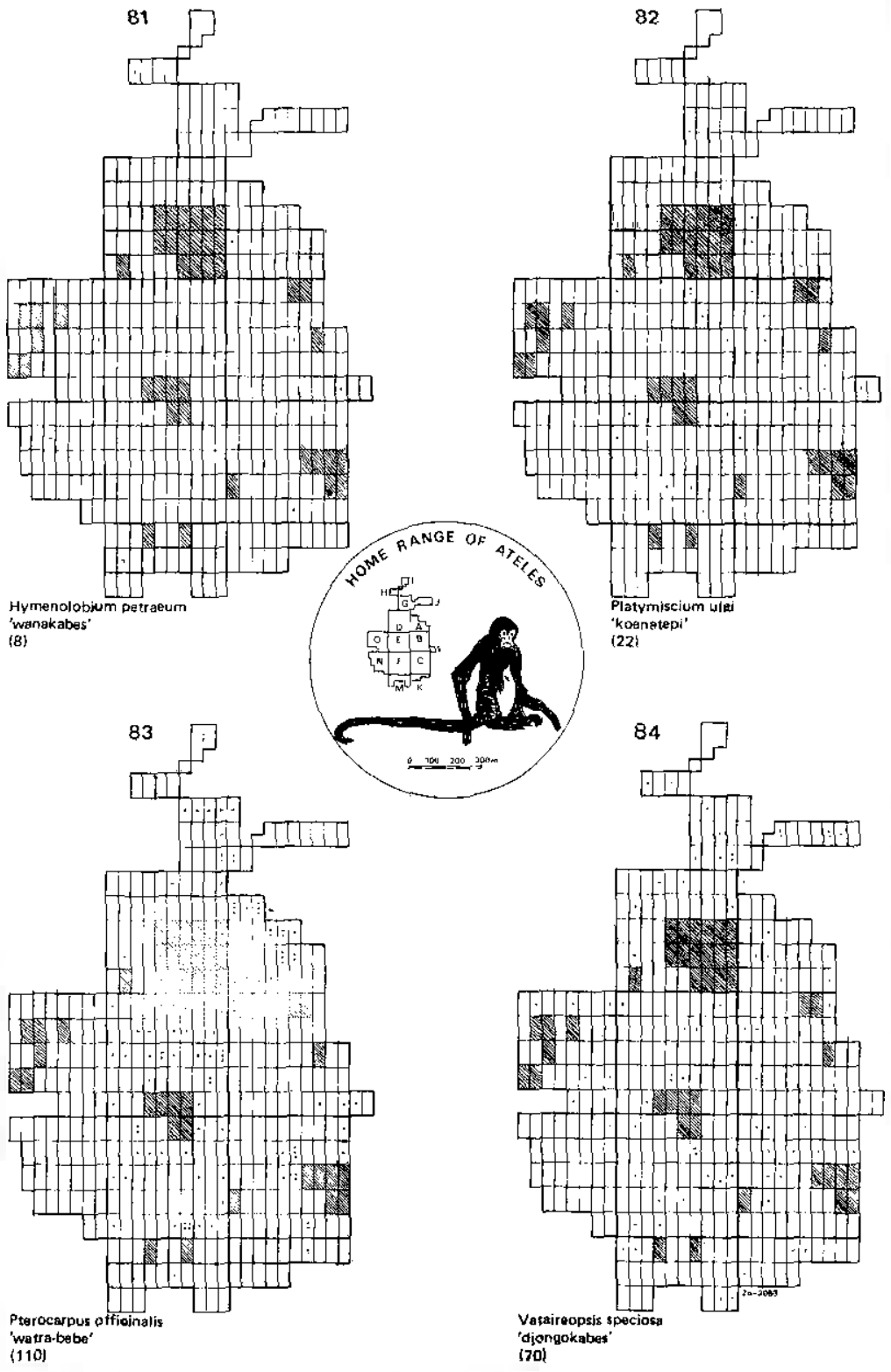


HOME RANGE SHOWING DISTRIBUTION AND DENSITY OF FOOD PLANTS"

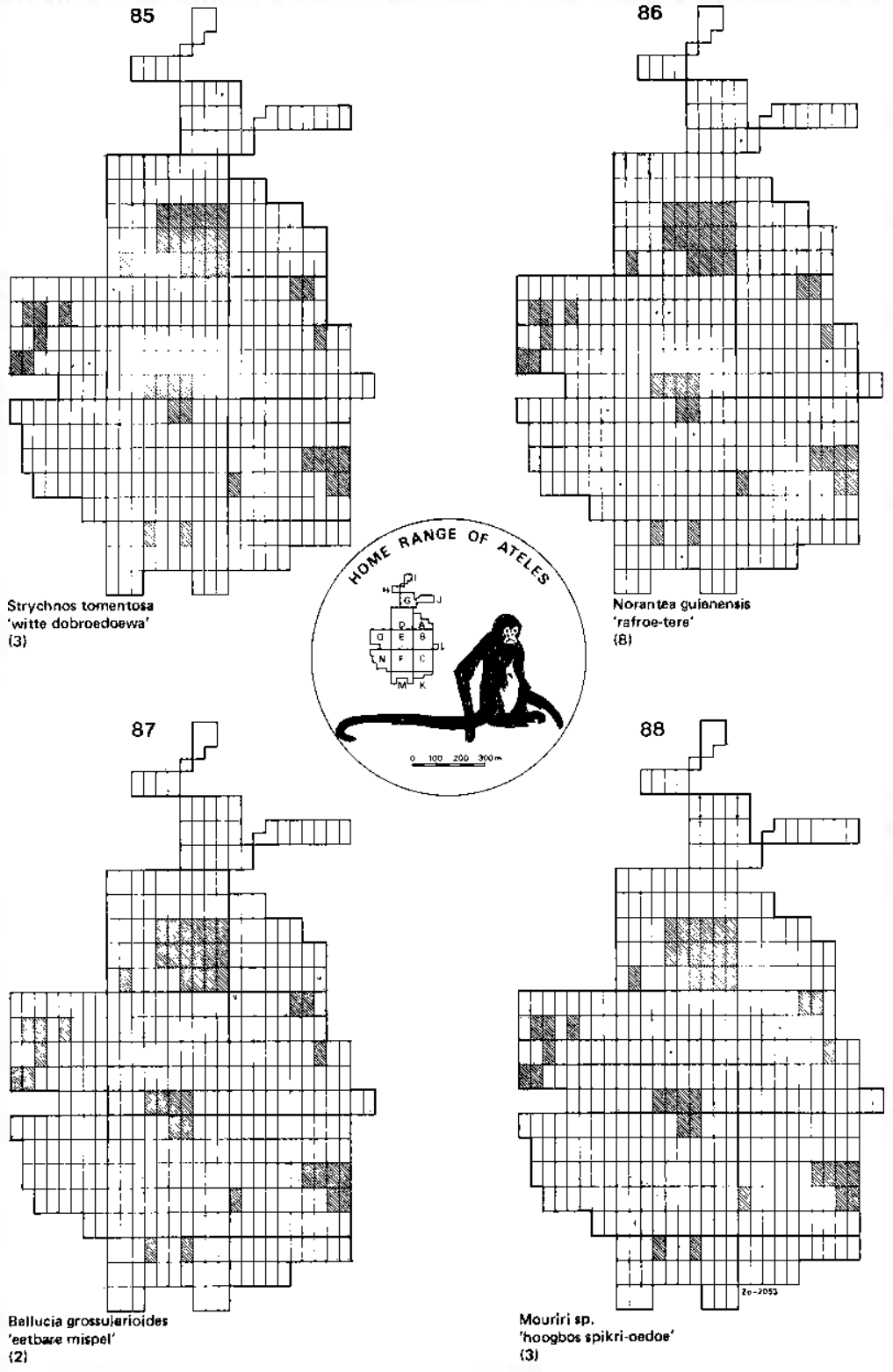


HOME RANGE SHOWING DISTRIBUTION AND DENSITY OF FOOD PLANTS

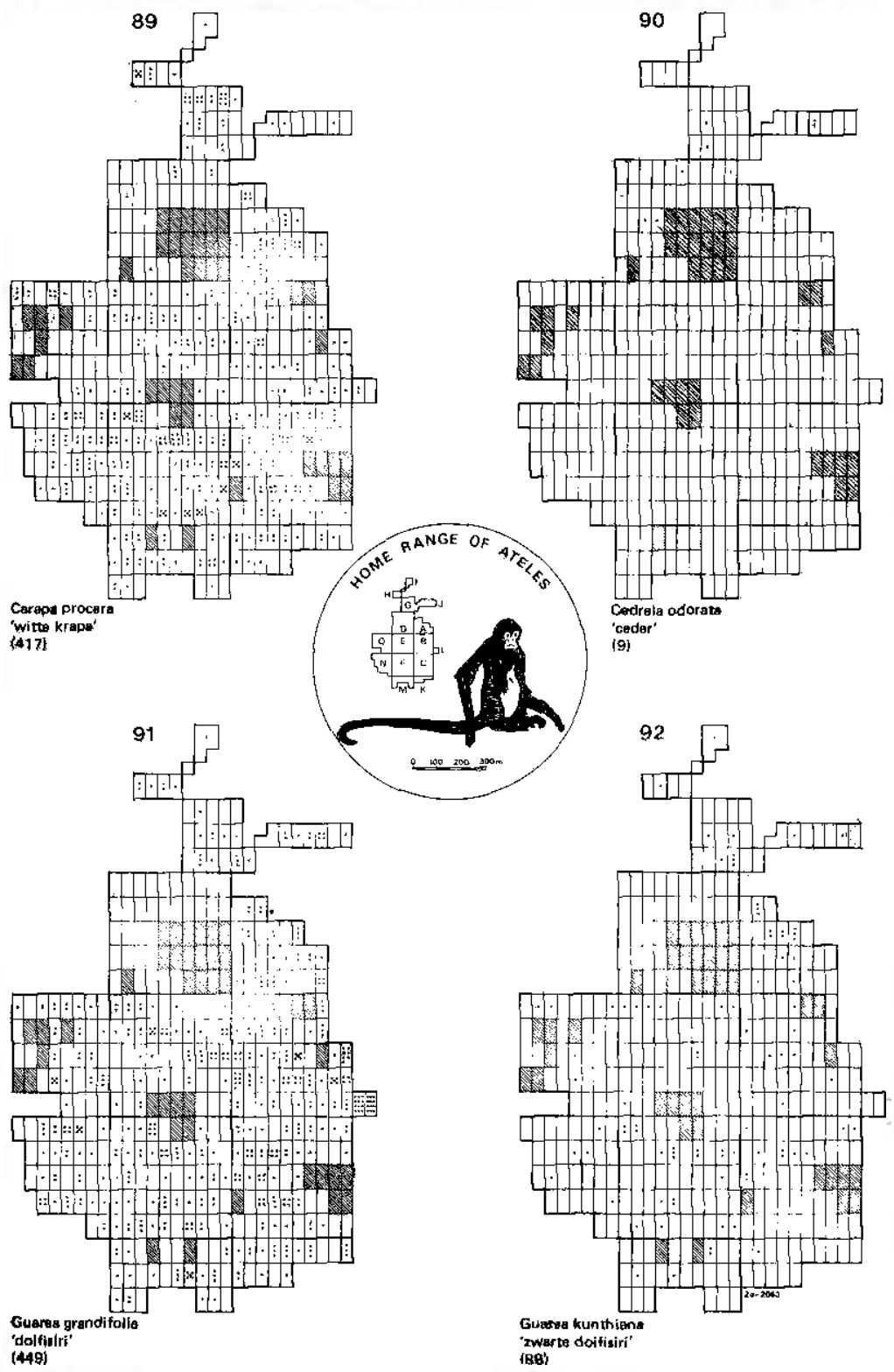

Habitat... 
HOME RANGE SHOWING DISTRIBUTION AND DENSITY OF FOOD PLANTS

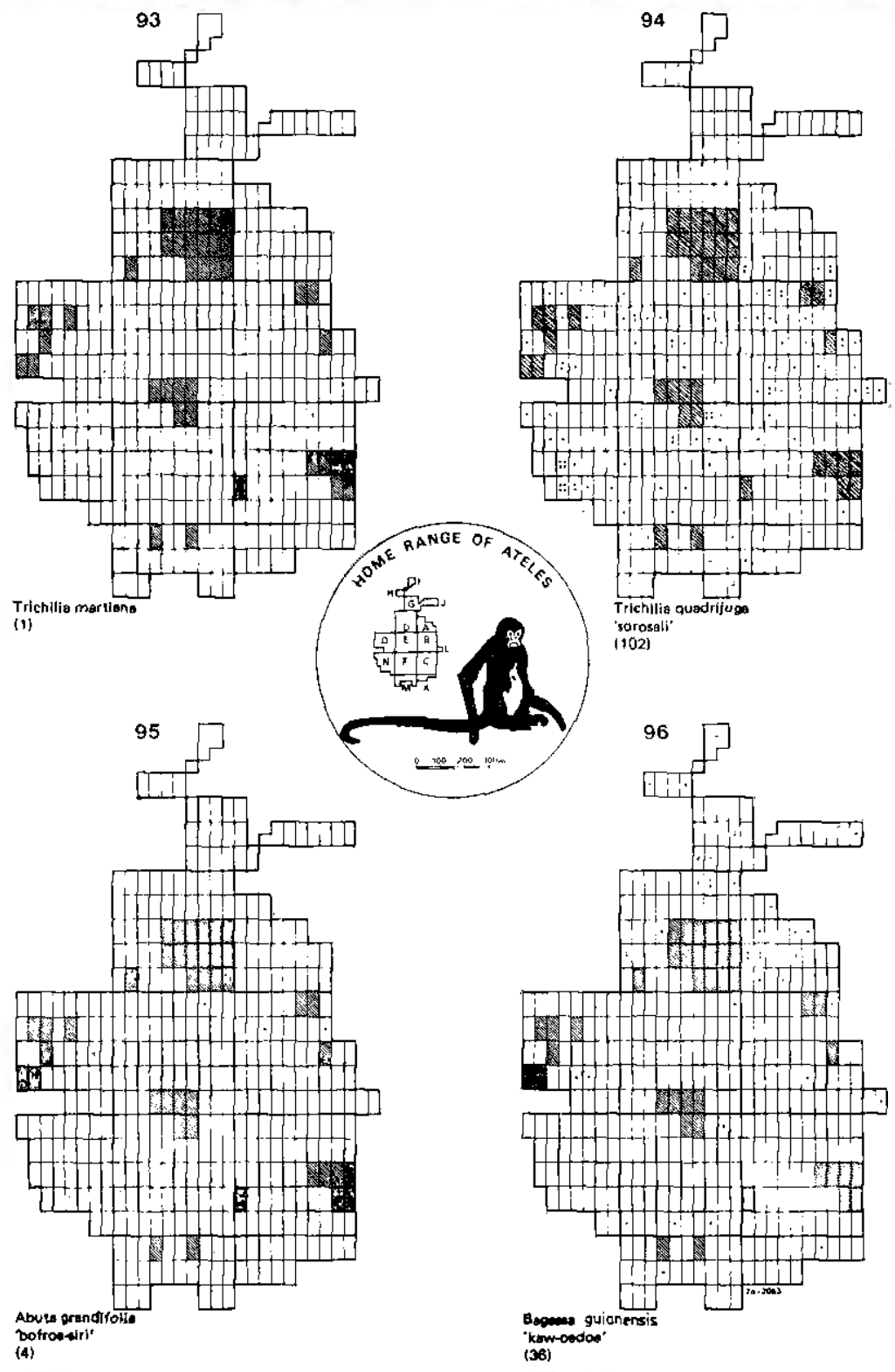


HOME RANGE SHOWING DISTRIBUTION AND DENSITY OF FODD PLANTS

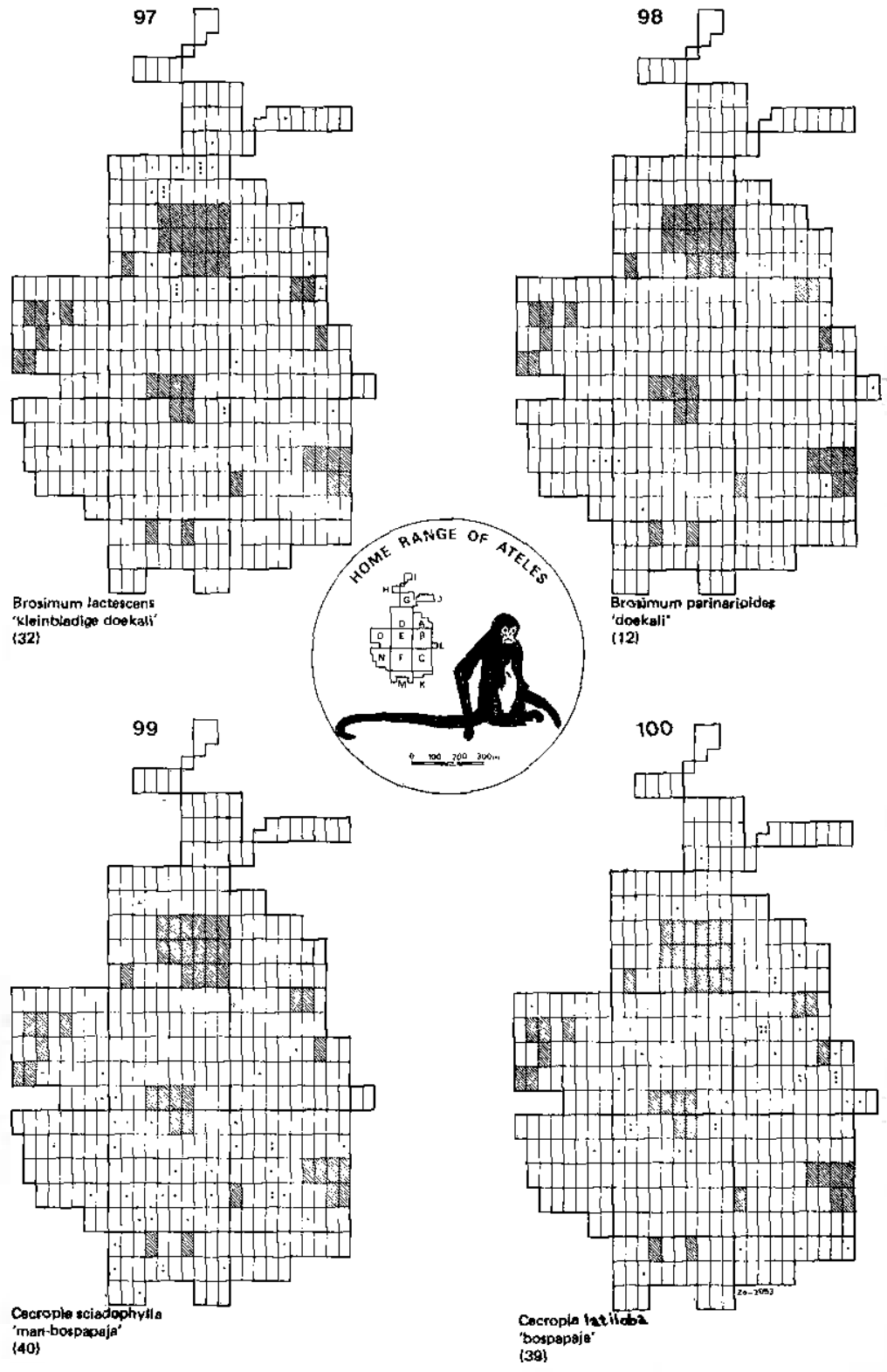

Habitat.. 
HOME RANGE SHOWING DISTRIBUTION AND DENSITY OF FOOD PLANTS

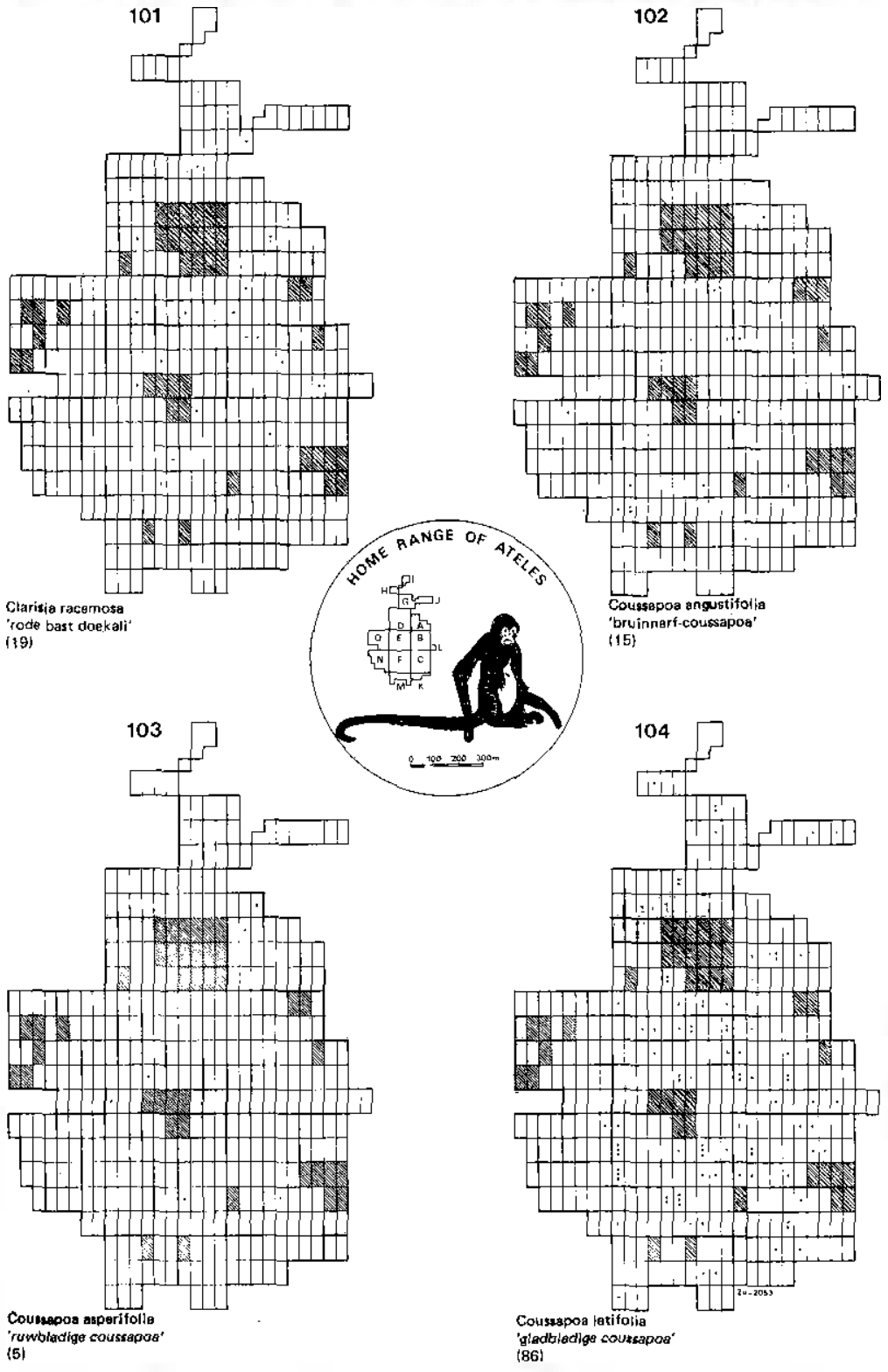


HOME RANGE SHOWING DISTRIBUTION AND DENSITY OF FOOD PLANTS

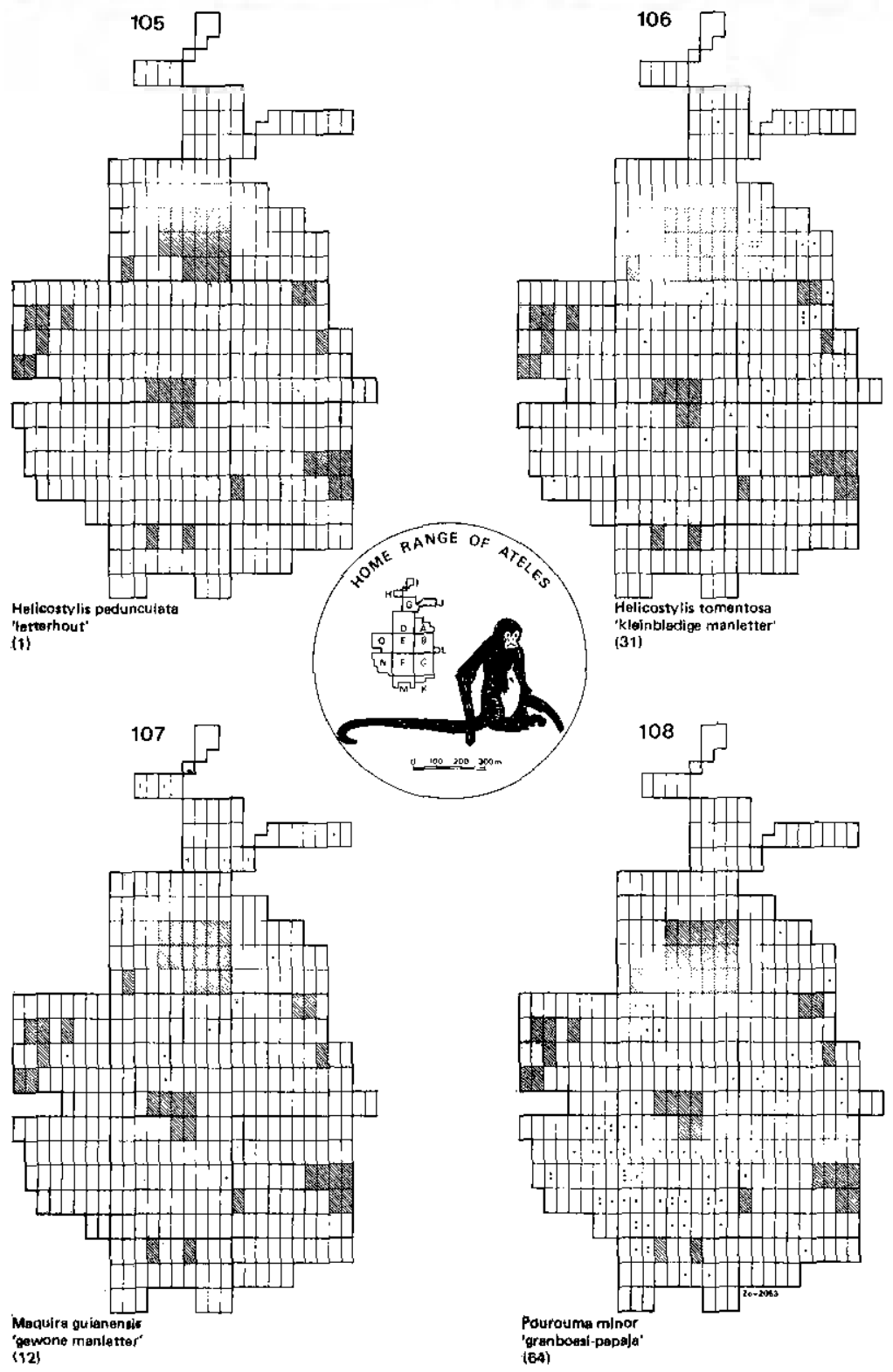

Habltat... 
HOME RANGE SHOWING DISTRIBUTION AND DENSITY OF FOOD PLANTS

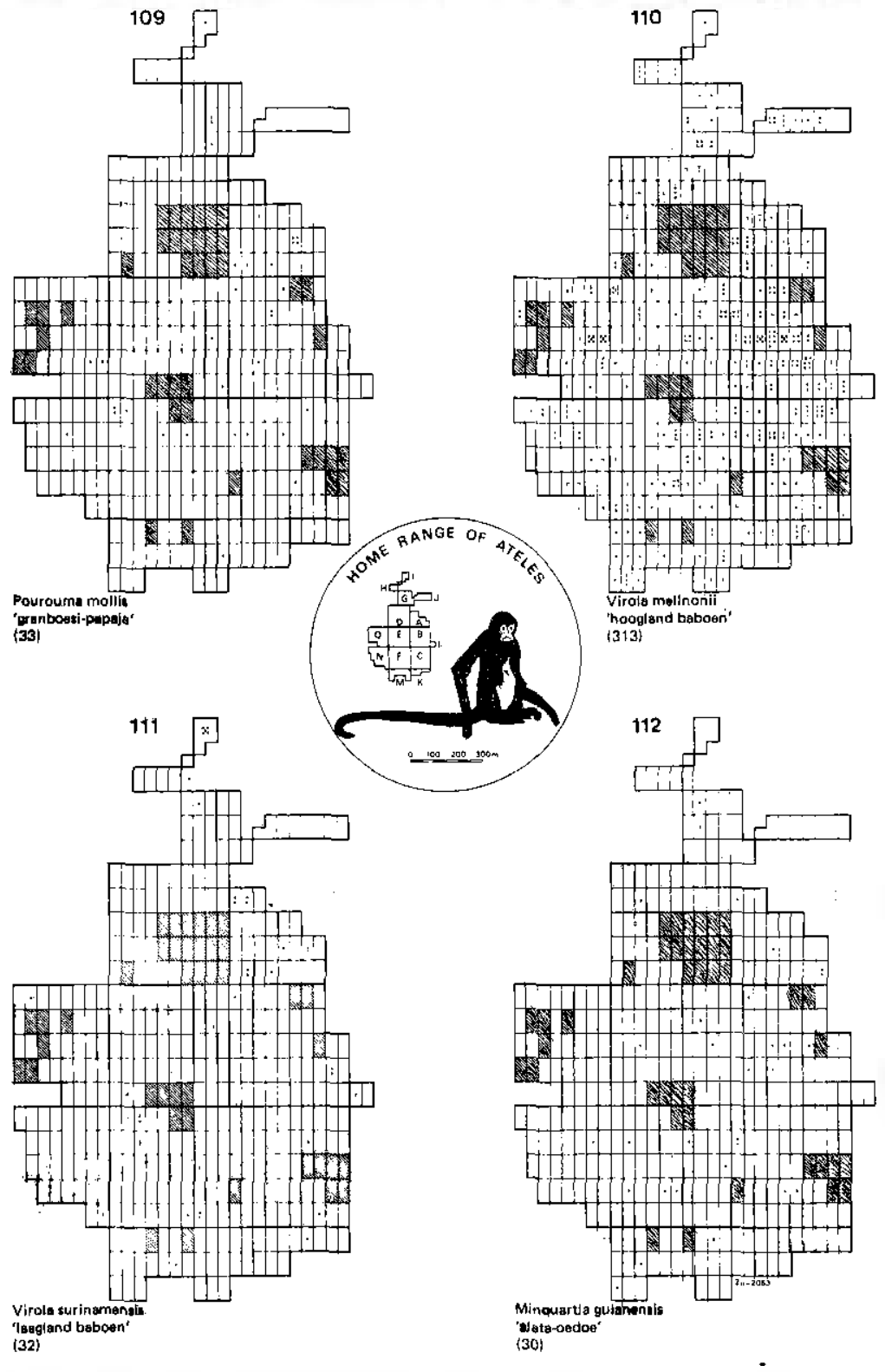


HOME RANGE SHOWING DISTRIBUTION AND DENSITY OF FOOD PLANTS

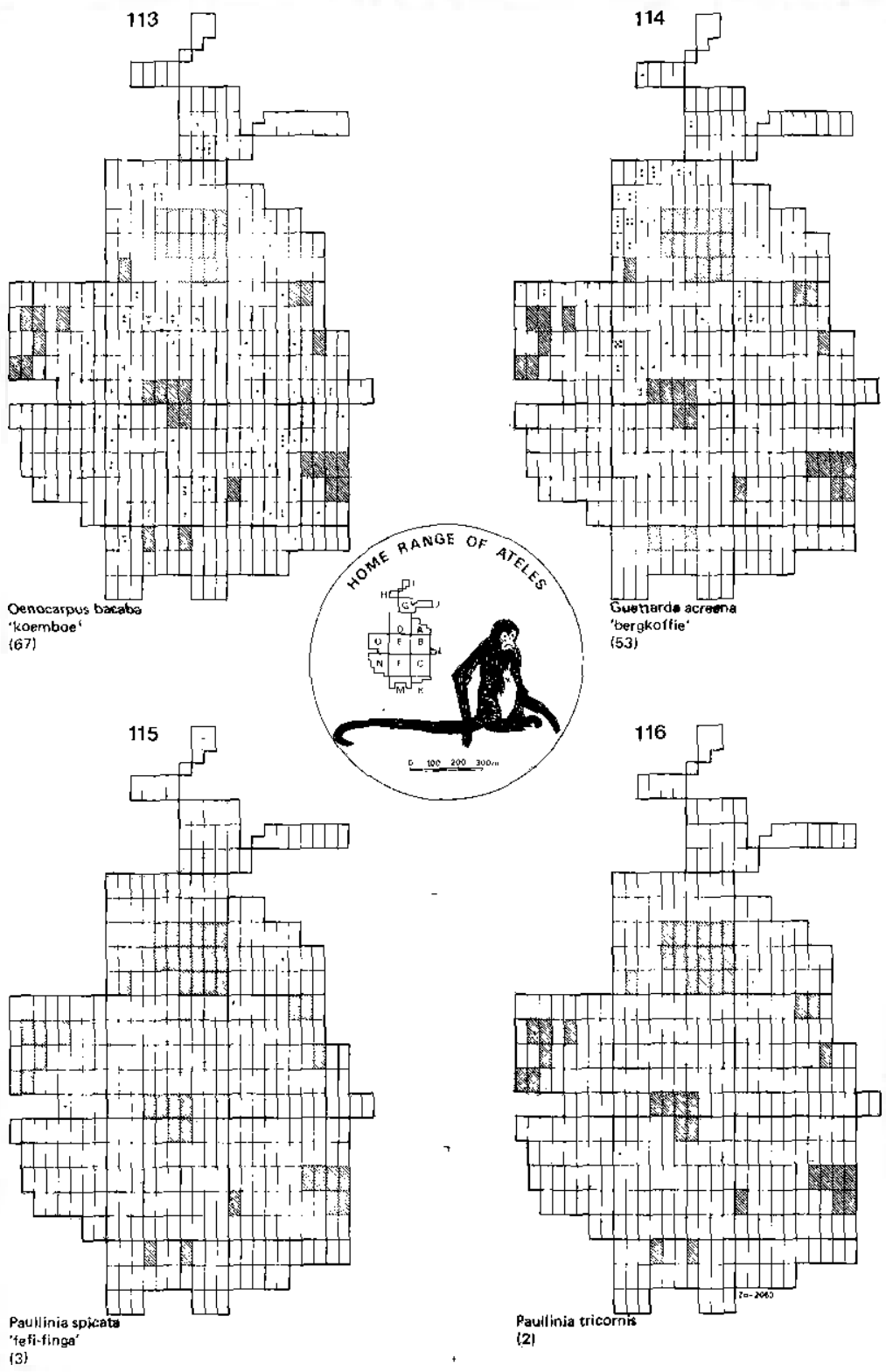


HOME RANGE SHOWING DISTRIBUTION AND DENSITY OF FOOD PLANTS

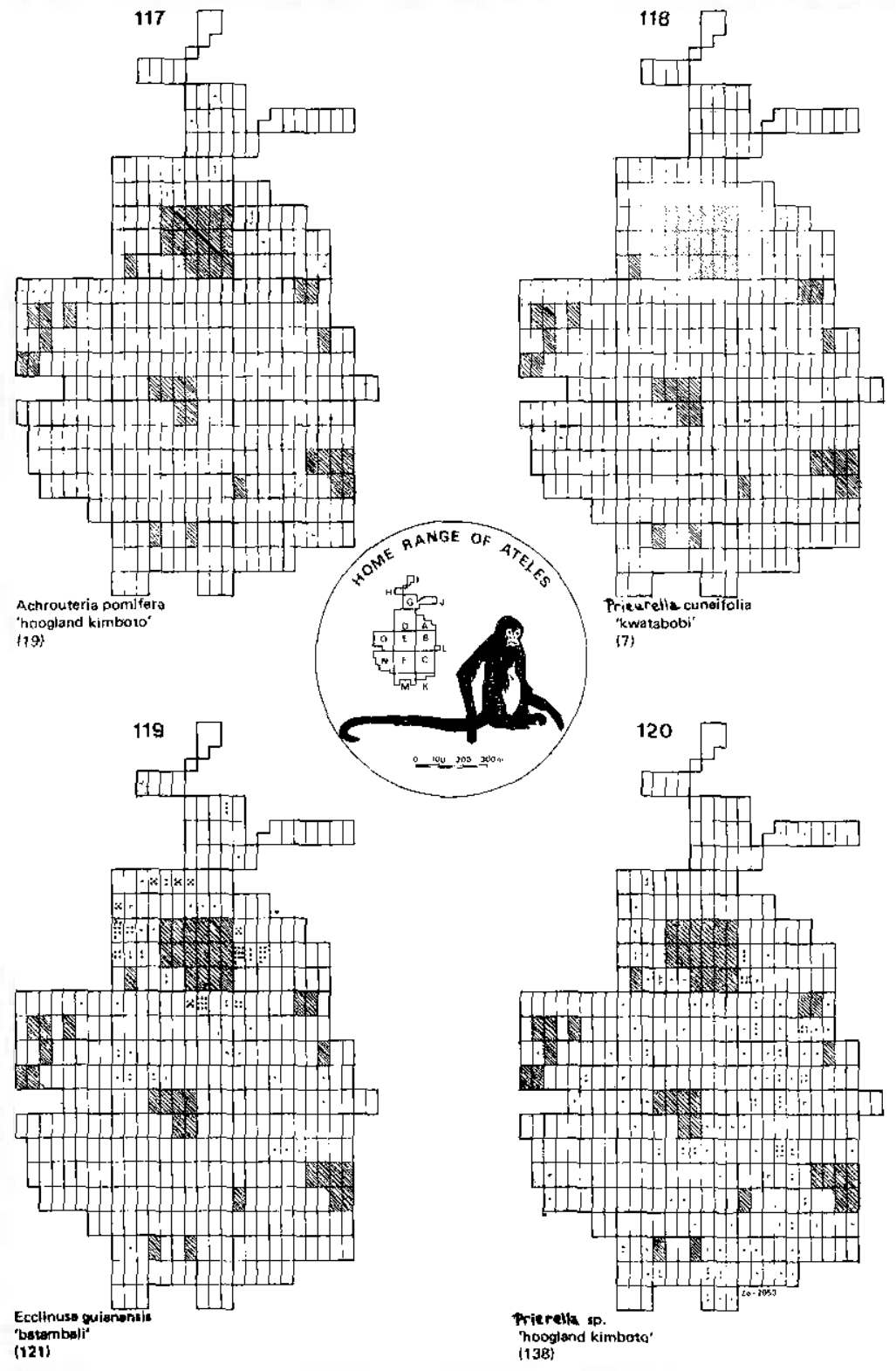


HOME RANGE SHOWING DISTRIBUTION AND DENSITY OF FOOD PLANTS

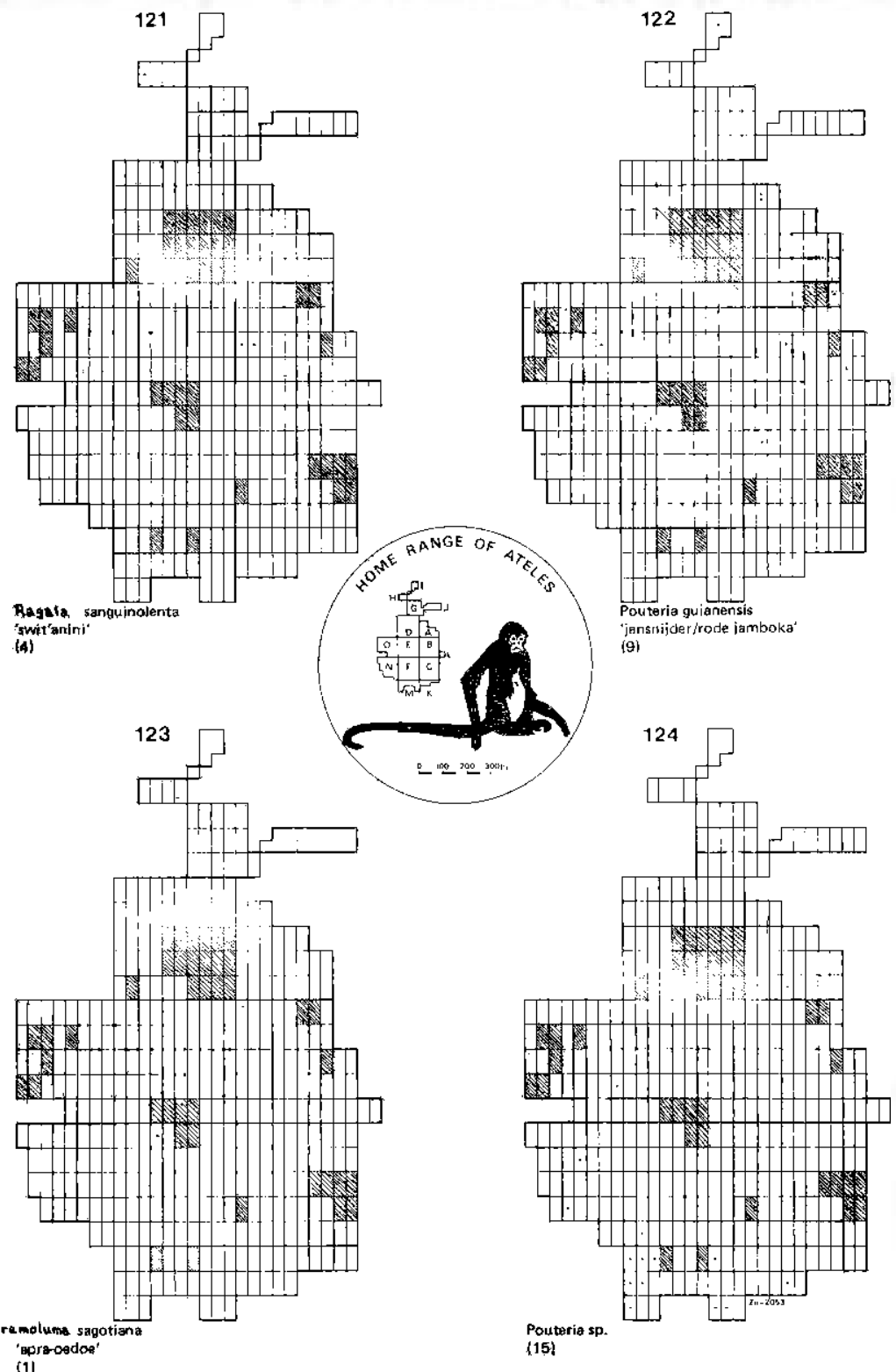
(1)

Habitat... 
HOME RANGE SHOWING DISTRIBUTION AND DENSITY OF FOOD PLANTS

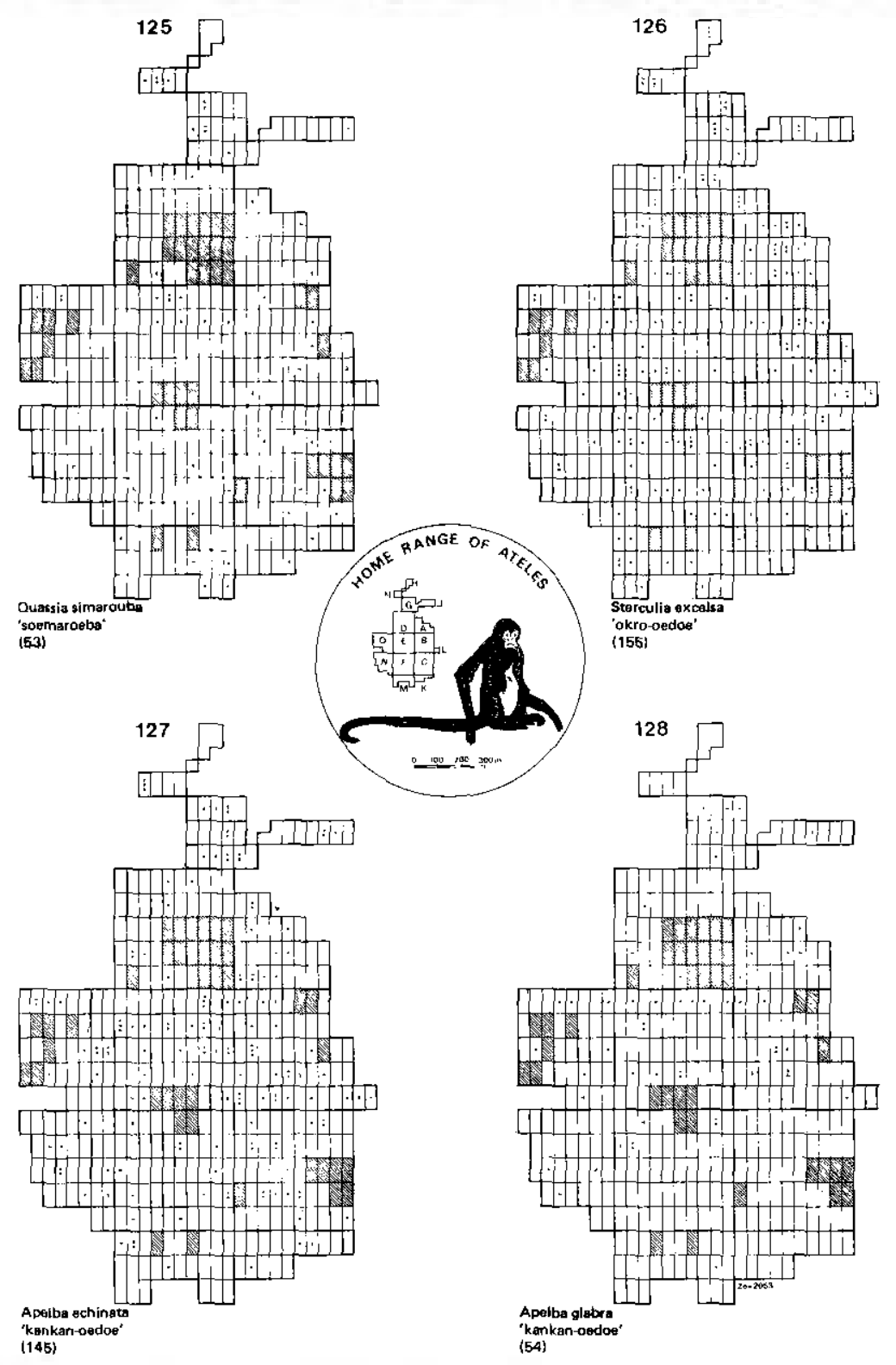


HOME RANGE SHOWING DISTRIBUTION AND DENSITY OF FOOD PLANTS

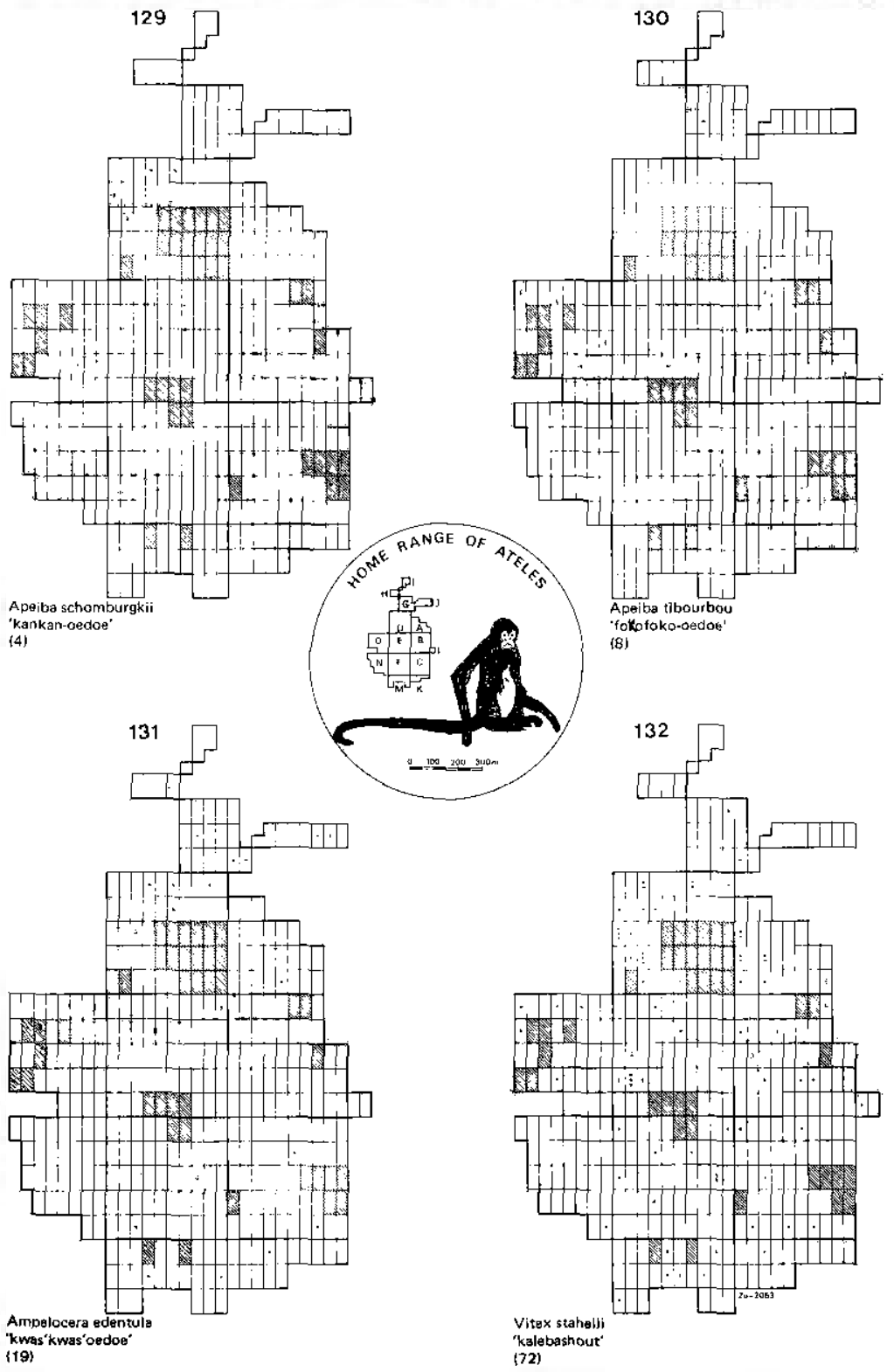

Habitat,.. 
HOME RANGE SHOWING DISTRIBUTION AND DENSITY OF FOOD PLANTS

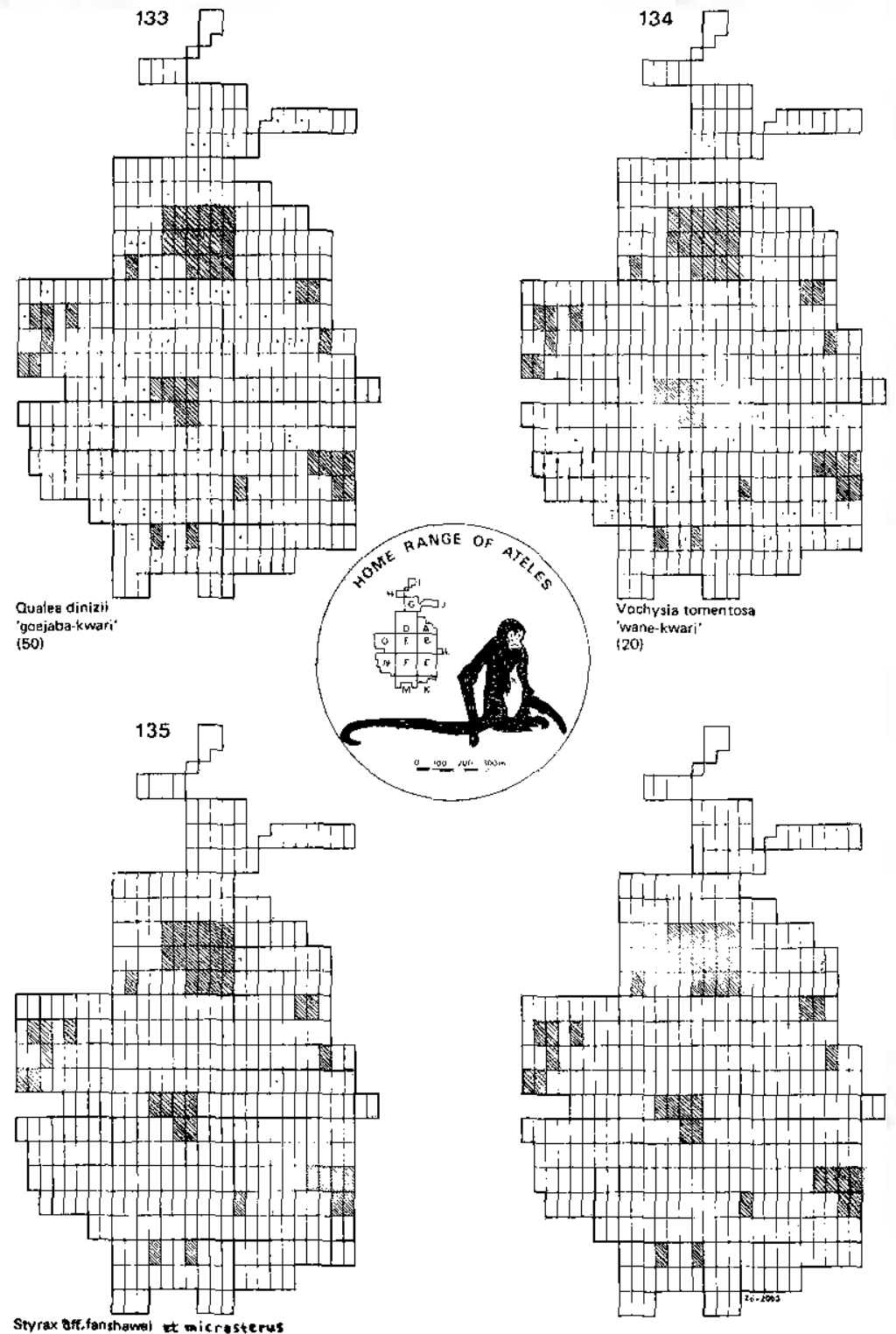

Styrax aff,fanshamal te microsterus

(2) 